\title{
Acoustic Cavitation and Sonochemistry
}

\section{Laura Stricker}




\title{
ACOUSTIC CAVITATION AND SONOCHEMISTRY
}

\author{
Laura Stricker
}




\section{Samenstelling promotiecommissie:}

Prof. dr. G. van der Steenhoven (voorzitter) Universiteit Twente Prof. dr. Detlef Lohse (promotor) Universiteit Twente

Prof. dr. Andrea Prosperetti (promotor) Universiteit Twente

Prof. dr. ir. D. C. Nijmeijer Universiteit Twente

Prof. dr. ir. L. Lefferts Universiteit Twente

Prof. dr J. G. E. Gardeniers Universiteit Twente

Prof. dr J. T. F. Keurentjes Technische Univ. Eindhoven

The work in this thesis was carried out at the Physics of Fluids group of the Faculty of Science and Technology of the University of Twente. It is part of the research programme of the Foundation for Fundamental Research on Matter (FOM), which is financially supported by the Netherlands Organisation for Scientific Research (NWO).

Nederlandse titel:

Akoestische cavitatie en sonochemie

Publisher:

Laura Stricker, Physics of Fluids, University of Twente, P.O. Box 217, 7500 AE Enschede, The Netherlands

http://pof.tnw.utwente.nl

stricker18306@gmail.com

Cover design: Laura Stricker

Cover illustration: Merging bubble clusters in a sonochemical reactor driven at high power (see Chapter 3).

(c) Laura Stricker, Enschede, The Netherlands 2013

No part of this work may be reproduced by print photocopy or any other means without the permission in writing from the publisher

ISBN: 978-90-365-3500-7 


\section{ACOUSTIC CAVITATION AND SONOCHEMISTRY}

\section{PROEFSCHRIFT}

ter verkrijging van

de graad van doctor aan de Universiteit Twente,

op gezag van de rector magnificus,

Prof. dr. H. Brinksma,

volgens besluit van het College voor Promoties

in het openbaar te verdedigen

op woensdag 16 januari 2013 om 12.45 uur

door

Laura Stricker

geboren op 16 juni 1983

te Turijn, Italie 
Dit proefschrift is goedgekeurd door de promotors:

Prof. dr. rer. nat. Detlef Lohse

$\&$

Prof. dr. Andrea Prosperetti 


\section{Contents}

1 Introduction 1

1.1 Cavitation and sonochemistry: a brief overview . . . . . . . . . . 1

1.2 Guide through the chapters ............... 5

2 Thermal models for acoustically driven bubbles 13

2.1 Introduction . . . . . . . . . . . . . . . . . . . . . . . . . . . . . .

2.2 Summary of the models . . . . . . . . . . . . . . . . . . . . . . 15

2.3 Numerical method . . . . . . . . . . . . . . . . . 18

2.4 Results . . . . . . . . . . . . . . . . . 20

2.5 Summary and conclusions . . . . . . . . . . . . . 29

3 Ultrasound artificially nucleated bubbles and their sonochemical radical $\begin{array}{ll}\text { production } & \mathbf{3 7}\end{array}$

3.1 Introduction . . . . . . . . . . . . . . . . . . . 38

3.2 Materials and Methods . . . . . . . . . . . . . . . . . 39

3.3 Results and Discussion ... . . . . . . . . . . . . . . 43

3.4 Summary and Conclusions . . . . . . . . . . . . 65

4 Radical production inside an acoustically driven microbubble $\quad 73$

4.1 Introduction . . . . . . . . . . . . . . . . . . . 74

4.2 Model . . . . . . . . . . . . . . . . . 76

4.3 Results . . . . . . . . . . . . . . . . . . . 78

4.4 Summary and conclusions ................... 95

5 Bubble growth by gas diffusion $\quad 103$

5.1 Model . . . . . . . . . . . . . . . . . 103

5.2 Validation: analytical test case . . . . . . . . . . . . . 106

5.3 Bubble nucleating next to a wall . . . . . . . . . . . . . 110 
6 The role of gas in ultrasonically driven vapor bubble growth 117

6.1 Introduction . . . . . . . . . . . . . . . . . 117

6.2 Materials and Experimental Methods . . . . . . . . . . . . . . . 119

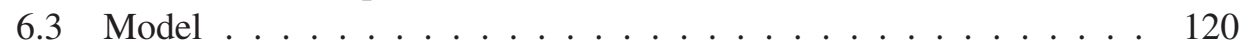

6.4 Results . . . . . . . . . . . . . . . . . . . . . . . . . . . . . . . 125

6.5 Summary and conclusions ................. 131

7 Oscillations of a meniscus of a gas filled cavity 135

7.1 Introduction . . . . . . . . . . . . . . . . . 135

7.2 Model . . . . . . . . . . . . . . . . . . 137

7.3 Numerical method . . . . . . . . . . . . . . . . . . . . . . . . . . . . . . . . . 138

7.4 Validation . . . . . . . . . . . . . . . . . 143

7.5 Results . . . . . . . . . . . . . . . . . . . . . . . . . . . . . . . . . . . . . . . . . . . . .

7.6 Summary . . . . . . . . . . . . . . . . . . . . . . . . . . . . . . . . . . 154

7.7 Appendix A . . . . . . . . . . . . . . . 155

8 Interacting bubble clouds and their sonochemical production $\quad 167$

8.1 Introduction . . . . . . . . . . . . . . . . . . . . . . . . . . . . 168

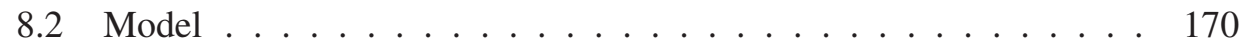

8.3 Results . . . . . . . . . . . . . . . . . . . . . . . . . . . . . . . 175

8.4 Conclusions .......................... 185

9 Conclusions 191

9.1 Conclusions and outlook . . . . . . . . . . . . . . 191

$\begin{array}{ll}\text { Summary } & 197\end{array}$

$\begin{array}{lr}\text { Samenvatting } & 199\end{array}$

$\begin{array}{ll}\text { Acknowledgements } & 201\end{array}$

$\begin{array}{ll}\text { About the author } & 203\end{array}$ 


\section{1 \\ Introduction}

\subsection{Cavitation and sonochemistry: a brief overview}

The word "cavitation" comes from the Latin word "cavus", hollow, and it was first coined by R. E. Froude. Cavitation is the creation of cavities inside a liquid, as a consequence of an abrupt decrease of the cohesive forces of the liquid itself, due to a rapid pressure drop. Although the phenomenon was already predicted by Euler in 1754 [1], the first real investigation dates back to 1895, when British torpedo boats were found to suffer from erosion and vibration associated with bubble formation [2]. The problem was temporarily solved by using slower and bigger propellers, but it came again to the attention in 1904, with the increase of propellers velocity. On request of the Royal Navy, Lord Rayleigh started to investigate the phenomenon and developed his pioneer work on the problem of the collapse of an empty cavity inside a liquid [3], thus providing the theoretical foundation for cavitation studying.

Some of the systems commonly adopted to induce cavitation are: the generation of a water flow through a local constriction, fast rotating propellers, the introduction of superheated steam into water and ultrasound driving. In the present work we will focus on the latter. Ultrasound is sound with a frequency above the human hearing threshold $(20 \mathrm{kHz})$. Sound is constituted by rarefaction and compression waves through a medium. In an ultrasonic apparatus this pressure wave is generated by means of a transducer, a device that converts one form of energy (e.g. electrical) to 


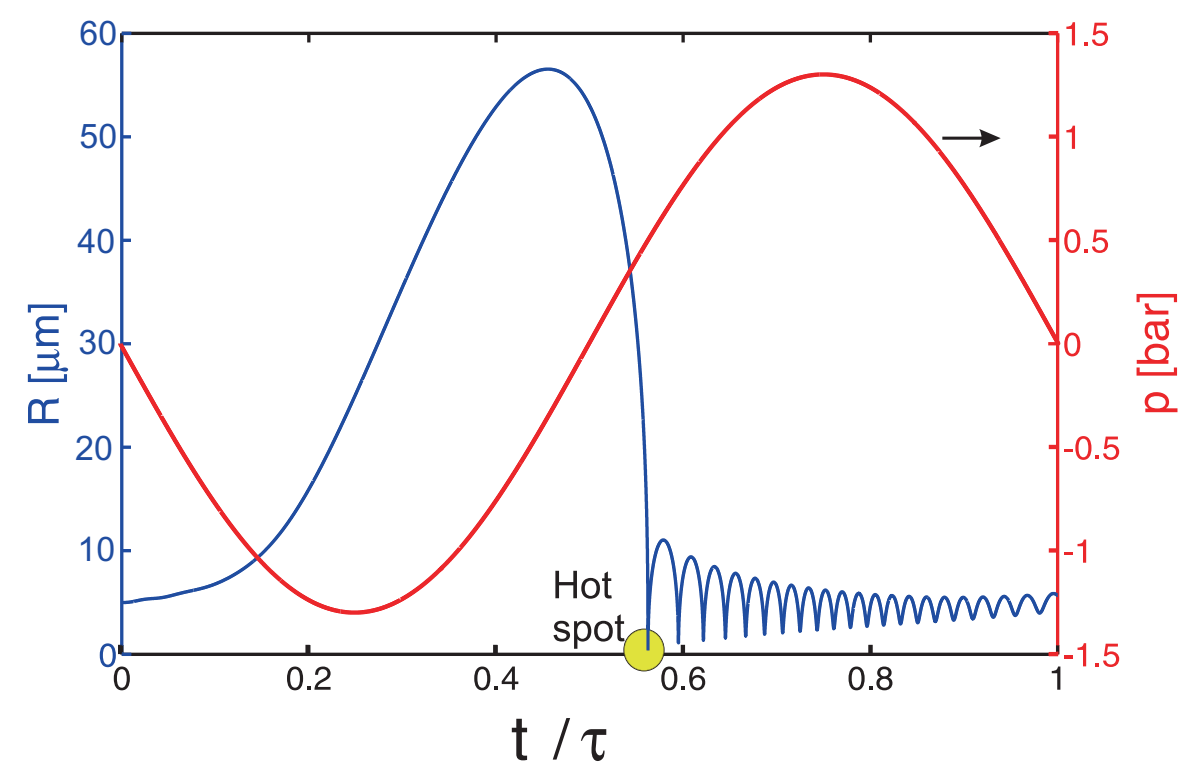

Figure 1.1: Radial dynamics (blue) of a bubble undergoing non-linear oscillations and the driving pressure (red). Parameters: $R_{0}=5 \mu \mathrm{m}, P_{a}=1.3 \mathrm{bar}, f=20 \mathrm{kHz}$

another (e.g. mechanical). The response of a bubble undergoing an acoustic forcing is governed by the well-known Rayleigh-Plesset equation $[4,5]$ and it can be of two different kinds, qualitatively speaking. For low driving amplitudes the bubble oscillates gently, like a harmonic oscillator (non-inertial cavitation) while, as the driving pressure increases and the dynamic Blake threshold is overcome [4, 6], it exhibits a nonlinear behavior (inertial cavitation). An example of a bubble undergoing nonlinear oscillations is shown in Fig.1.1. A first initial expansion is produced in the negative pressure phase, then followed by an abrupt collapse, similar to the Rayleigh collapse [3] as the pressure increases halting the expansion, and by a series of damped rebounces with essentially the eigenfrequency of the bubble (Minnaert frequency $[4,7])$. The abrupt collapse is associated with a huge energy focusing and with a sudden temperature increase, as the heat has no time to escape from the bubble and the process is almost adiabatic. Many interesting phenomena are associated to such an energy focusing: shock waves [8], jets (when the collapse happens next to a surface) $[9,10]$, emission of light (sonoluminescence) [11] and chemical reactions (sonochemistry) [12].

The chemical effects of ultrasound were reported for the first time by Wood and Loomis in 1927 [13], but the topic was then set aside for the following 50 years. It 
came to a renewed attention to the scientific community when inexpensive and reliable high-intensity ultrasound laboratory equipment started to appear, in the 1980s, with ultrasonic cleaning baths.

As a general definition, sonochemistry is the use of cavitation for achieving a chemical conversion. Chemical effects of cavitation can take place in different regions: in the liquid bulk, due to mechanical effects of the shear forces created by the shockwave at collapse [14] (mixing and suspended particle fragmentation), inside the bubble, which acts as a microreactor, and in the liquid shell around it. As we showed before, implosion of microbubbles (5-20 $\mu \mathrm{m}$ in size) can generate localized extreme temperatures of $10000 \mathrm{~K}$ and pressures up to $1000 \mathrm{bar}$, the conditions of the surrounding liquid remaining ambient [14-19]. Therefore high temperature chemical conversions can occur at ambient conditions, thus giving origin to highly reactive radical species. For example, when the liquid is water, $\mathrm{H}_{2} \mathrm{O}$ vapor is present inside the bubble. Its dissociation creates $\mathrm{OH}, \mathrm{O}$ and $\mathrm{H}$ radicals, $\mathrm{H}_{2} \mathrm{O}_{2}$ and $\mathrm{O}_{3}$. These chemical products then diffuse outside the bubble, dissolving in the surrounding liquid and easily oxidizing solutes. If volatile solutes are dissolved in the liquid, they enter the bubbles by evaporation and they are dissociated as well [20]. The reaction products can be used for synthesis of fine chemicals, food ingredients or pharmaceuticals, for degradation of water contaminants [21-24], for textile processing [25] or for cells disruption [26].

However, despite its vast potentiality, industrial application of sonochemistry has always been limited both by the difficulty of controlling the process and the energy inefficiency of large scale sonochemical reactors. In sonochemical devices, energy losses are difficult to prevent due to insufficient focus of energy transfer to the microbubble and to the bubble/bubble and bubble/wall interactions. Moreover erosion of the sonicator surfaces can emerge at the high operating power required for industrial-scale applications.

Sonochemical reactors currently developed are of two types: horn-type and standing wave-type. In horn-type reactors (Fig. 1.2a), an ultrasonic horn is immersed inside the liquid container and it radiates a quasi-spherical wave; thus a cloud of bubbles form around it. The driving pressure amplitude near the horn tip can reach values up to 10 atm [27], but it considerably decays and eventually vanishes as the distance from the horn tip increases. In standing wave reactors (Fig. 1.2b), the transducer is glued to the external surface of the container, either on the bottom or on the side walls, and it generates a standing wave inside the liquid. When the piezo is glued at the bottom (as in Fig. 1.2b), a pressure node will be located at the air-liquid interface. In this kind of reactors, the driving pressure amplitude is typically much lower than in horn reactors. Moreover, at high driving amplitude, the bubbles neither nucleate 


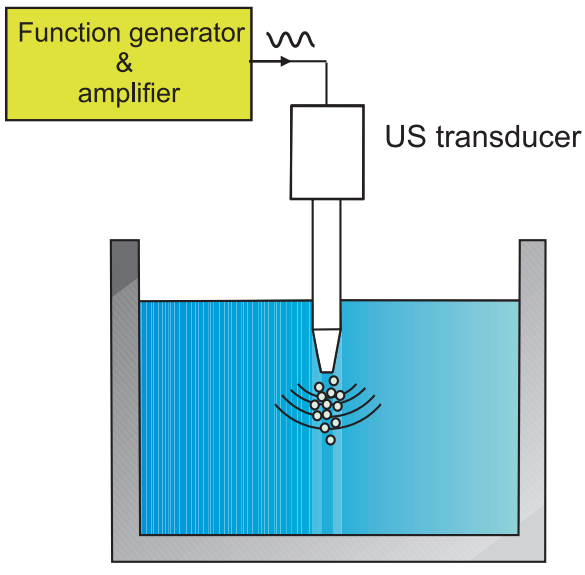

a)

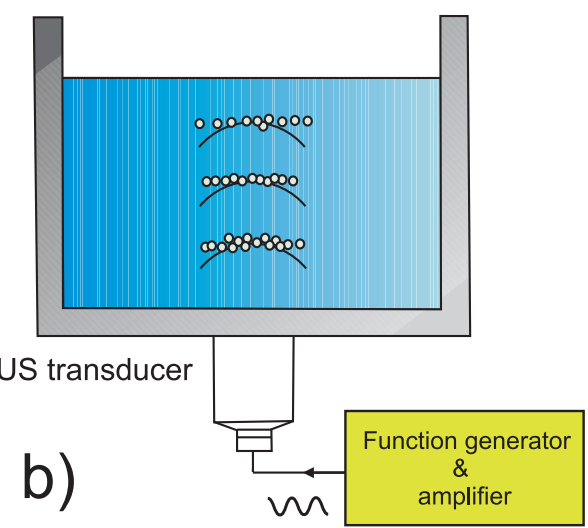

Figure 1.2: Different kinds of sonochemical reactors: horn-type reactor (a) and standing wave reactor (b).

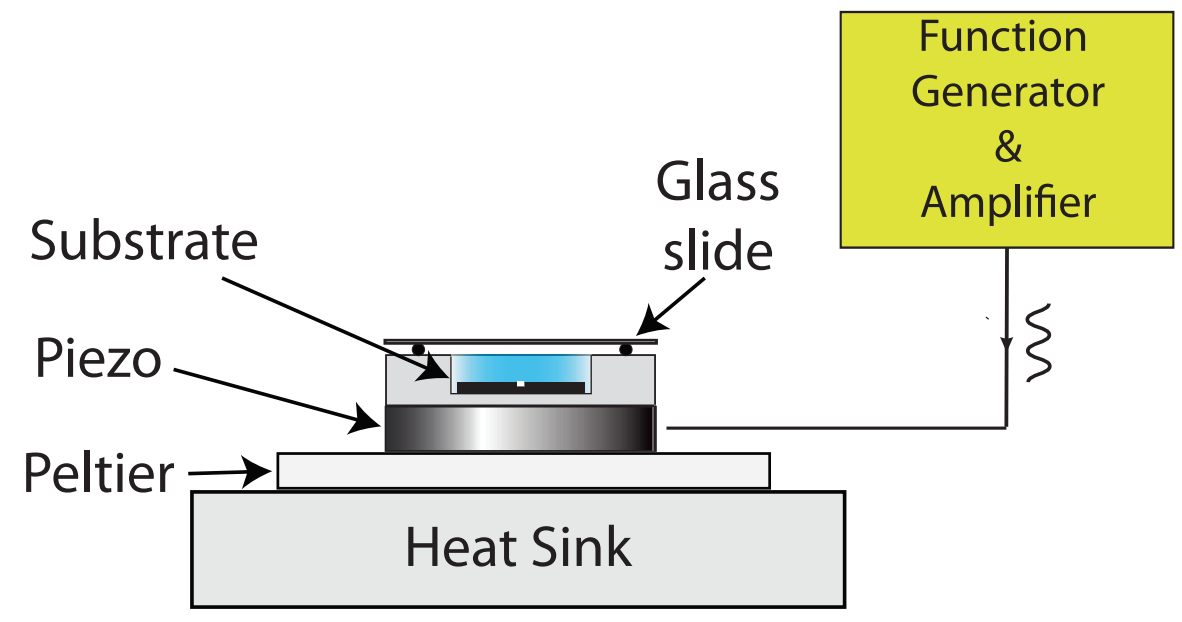

Figure 1.3: New efficient sonochemical microreactor of the kind described in Ref.[28, 29], based on the heterogeneous nucleation of bubbles from micromachined crevices over a silicon substrate 
nor gather where the pressure amplitude is maximal (antinodal planes), as expected, but tend to nucleate nearby the nodal planes and to cluster at regions in between the nodal and the antinodal planes, forming "jellyfish" structures [30].

When the project started, the challenge was to gain full control over the cavitation process and to improve the energy efficiency of the process, by miniaturizing sonochemical reactors. The leading idea was to increase the number of produced bubbles without loosing control of the process. It is known that cavitation can be favored by the presence of "weaknesses" inside the liquid (nucleation sites), where the tensile strength is lower. In the case of homogenous nucleation these weak points originate from thermal fluctuations, while in the case of heterogeneous nucleation, they originate from solid-liquid interfaces. Crevices, either on surfaces or on dispersed impurities, can entrap gas pockets thus favoring cavitation [7]. Bremond et al. $[31,32]$ showed that artificial crevices (pits) micromachined over a silicon surface can produce stable and monodisperse cavitation nuclei. Using this idea, Fernández Rivas et al [29, 33] developed a new kind of sonochemical microreactor of the standing wave type, where a silicon substrate with artificial micropits was placed at the bottom of the liquid cuvette (see Fig.1.3). Investigation with luminol showed that the bubbles ejected from the acoustically driven micropits were chemically active, as they produced $\mathrm{OH}$ radicals which reacted with luminol giving origin to light emission, process know as sonochemiluminescence (SCL) [34] (Fig. 1.4). This reactor presented an increase of the chemical yield of a factor 10 respect to the equivalent preexisting immersed-bath reactors and it currently represents the state of the art [29]. The present work is intended as a theoretical studying of that device, whose experimental investigation has been addressed in Ref. [28]. Some extensions to more fundamental aspects have also been addressed.

\subsection{Guide through the chapters}

The chemical reactivity depends on a number of experimentally tunable parameters, such as frequency, driving pressure, kind of solvent, liquid temperature, hydrostatic pressure and saturation conditions of the liquid. When one wishes to develop a comprehensive parametric study of the radical production, a full model would result rather complex and computationally expensive. Even neglecting the bubble-bubble interaction, i.e. in the case of single-bubble sonoluminescence, a complete description of the process should take into account spatial pressure and temperature distribution, mass and heat diffusion, evaporation/condensation phenomena, change in transport parameters due to thermal and compositional changes of the mixture, inertial effects and chemical reactions. Therefore the need of simplification. 


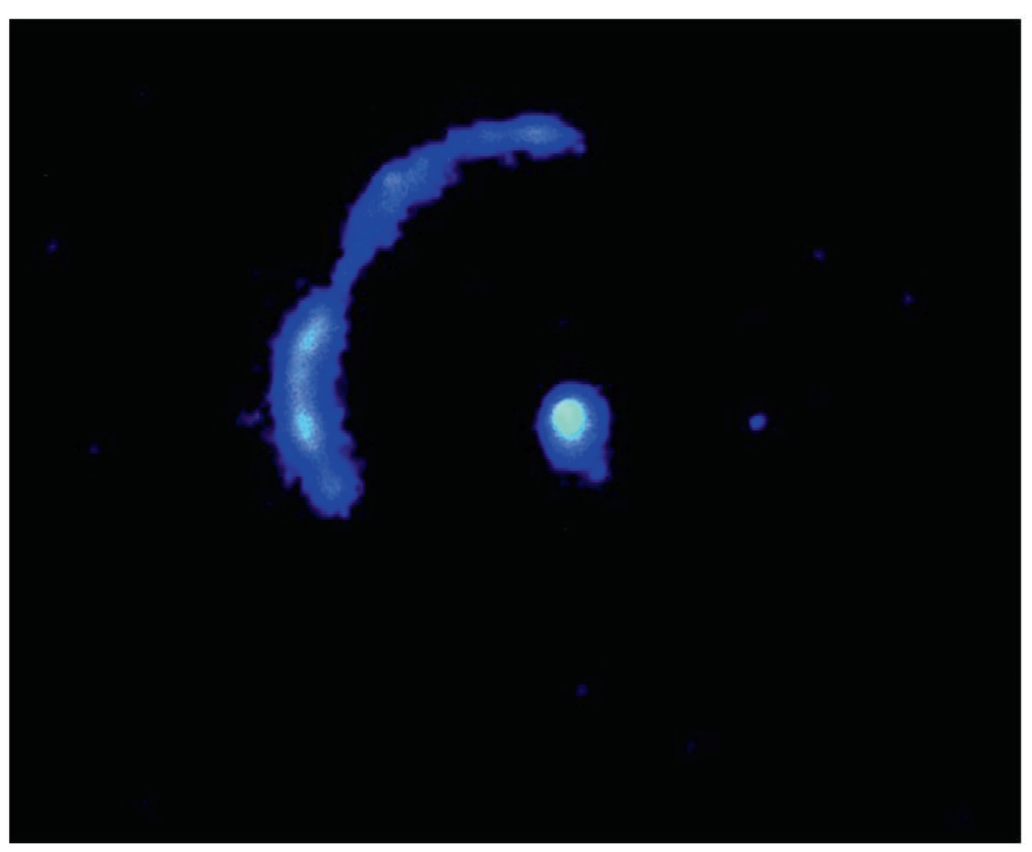

Figure 1.4: Area of enhanced radical production around one pit of a new reactor of the kind of Fig.1.3 [29]. The blue areas are produced by the light emission of luminol, reacting with the $\mathrm{OH}$ radicals generated at bubble collapse (sonochemiluminescence, SCL). Reproduced with permission from Ref.[28].

In Chap. 2, we validate a simplified ODE model for single-bubble sonoluminescence [35-37], based on the boundary layer assumption, by comparing it with a complete PDE model solving the heat advection-diffusion equation [38, 39]. While the latter takes into account the temperature field both outside and inside the bubble, the simplified model considers the bubble as thermally uniform. We focus on the peaks of temperatures and heat fluxes, because this is what governs the chemical kinetics, through Arrhenius law.

In Chap. 3, we characterize the sonochemical reactor described in Ref. [33], by means of the radial evolution of the bubble population that it produces. Using the simplified model validated in Chap.2, we evaluate immeasurable experimental parameters such as the pressure and we estimate the radical production in different configurations. 
In Chap. 4, we develop a parametric studying of the single-bubble radical production, by means of the simplified model validated in Chap. 2. We consider the case of transient cavitation, in order to match the experimental conditions of the reactor of Ref. [29, 33], where the bubbles were found to survive only few cycles due to abrupt splitting and recombination phenomena. In particular, we investigate the role of the frequency, the driving amplitude, the liquid temperature and the kind of gas dissolved inside the liquid, providing optimal working ranges. We also provide a further validation to the model, reproducing the explosive well known behavior of stoichiometric mixtures of $\mathrm{H}_{2}$ and $\mathrm{O}_{2}$.

In view of a future extension of the complete PDE model developed in Chap. 2, including both thermal and mass diffusion, evaporation and chemical reactions, in Chap. 5 we develop a model for bubbles growing by mass diffusion. We validate it comparing it both with the analytical solution in a test case and with experimental results regarding the growth of a diffusive a bubble attached to a pit. In order to compare the numerical results with the experimental ones, a geometrical correction is introduced to keep into account the disruption of the boundary layer due to the presence of the wall.

In Chap. 6 we develop a PDE model for vapor bubbles including both thermal and mass diffusion inside the liquid, verifying that, in the limiting case of pure mass diffusion, it gives the same results of the model of Chap. 5. We validate it by comparison with results from an acoustic droplet vaporization (ADV) experiment. In particular, we study the time evolution of an ultrasound driven vapor bubble of perfluoropentane (PFP) inside a droplet of the same liquid, immersed in a water medium superheated respect to the PFP boiling point. We show the fundamental role of gas diffusion in order to prevent the bubble recondensation at collapse.

In Chap. 7 we study the oscillations of a gas pocket trapped inside a micropits of the kind used in the reactor of Chap. 3. We develop a code based on the level set approach [40], to track the meniscus dynamics. In order to validate the pressure field evolution, we calculate the analytical solution of a mixed boundary problem, in the test case of flat meniscus, and we verify that it is in good agreement with the numerical results. We additionally validate the code against the analytic solution calculated by Gelderblom et $a l$, in the limiting case of Stokes and potential flow [41]. We address both the free and the forced oscillations of the meniscus, both far and close to a wall, deriving the eigenfrequency and the damping coefficient of the trapped gas pocket.

In Chap. 8 we investigate the collective behaviors of the bubble clusters originating in the microreactor of Chap. 3 , by incorporating the effect of the secondary Bjerknes forces inside the simplified model that we validated in Chap. 2. We study 
the transition between the different observed behaviors: clusters sitting upon their own pits, clusters pointing towards each other and clusters migrating towards the center point of the pit array. This last behavior is particularly undesired in sonochemical reactors as it was showed to be associated to lower chemical yields (see Chap. 3). We correlate the transition with different parameters, such as the size of clusters, the number and size of the bubbles, the driving pressure and the distance between the pits. We also show how the radical production is affected by the Bjerknes forces, providing a possible explanation of the observed phenomenon for which high applied powers do not improve the radical production, but sometimes even reduce it [33].

Chap. 9 forms the conclusion. It summarizes the modelling work which has been done, stressing the complexity of the investigated problem and the limitations of the adopted assumptions, but also the achieved milestones. 


\section{References}

[1] L. Euler, Histoire de l'Academie Royale des Sciences et Belle Letters (Memo.,R. 10, Berlin) (1754).

[2] S. W. Barnaby and S. J. Thornycroft, "Torpedo boat destroyers", Proc. Inst. Civ. Engrs. 122 (1895).

[3] L. Rayleigh, "On the pressure developed in a liquid during the collapse of a spherical cavity", Philos. Mag. 34, 94-98 (1917).

[4] T. G. Leighton, The acoustic bubble (Academic Press, London) (1994).

[5] M. S. Plesset, "Comment on 'Sonoluminescence from water containing dissolved gases' (J. Acoust. Soc. Am. 60, 100-103 (1976))”, J. Acoust. Soc. Am. 62, 470 (1977).

[6] S. Hilgenfeldt, M. P. Brenner, S. Grossmann, and D. Lohse, "Analysis of Rayleigh-Plesset dynamics for sonoluminescing bubbles”, J. Fluid Mech. 365, 171-204 (1998).

[7] C. E. Brennen, Cavitation and Bubble Dynamics (Oxford University Press, Oxford) (1995).

[8] R. Hickling and M. S. Plesset, "Collapse and rebound of a spherical bubble in water”, Phys. Fluids 7, 7-14 (1964).

[9] C. F. Naude and A. T. Ellis, "On the mechanism of cavitation damage by nonhemispherical cavities in contact with a solid boundary.", ASME, J. Basic Eng. 83, 648-656 (1961).

[10] Y. Tomita and A. Shima, "High-speed photographic observations of laserinduced cavitation bubbles in water", Acustica 71, 161-171 (1990).

[11] M. P. Brenner, S. Hilgenfeldt, and D. Lohse, "Single bubble sonoluminescence", Rev. Mod. Phys. 74, 425-484 (2002).

[12] K. Suslick and S. Doktycz, "Sounding out new chemistry", New Scientist 125, 50-53 (1990).

[13] R. W. Wood and A. L. Loomis, "The physical and biological effects of high frequency sound waves of great intensity”, Philos. Mag. 4, 414 (1927). 
[14] T. J. Mason and J. P. Lorimer, Applied sonochemistry, the uses of power ultrasound in chemistry and processing (Wiley-VCH, Weinheim) (2002).

[15] K. S. Suslick, “Sonochemistry”, Science 247, 1439-1445 (1990).

[16] K. S. Suslick, S. J. Doktycz, and E. B. Flint, “On the origin of sonoluminescence and sonochemistry”, Ultrasonics 28, 280-290 (1990).

[17] K. S. Suslick and G. J. Price, "Applications of ultrasound to materials chemistry”, Ann. Rev. Mat. Sci. 29, 295-326 (1999).

[18] L. A. Crum, T. J. Mason, J. L. Reisse, and K. S. Suslick, eds., See the articles in Sonochemistry and Sonoluminescence (Kluwer Academic Publishers, Dordrecht) (1999).

[19] K. S. Suslick and D. J. Flannigan, "Inside a collapsing bubble: Sonoluminescence and the conditions during cavitation”, Ann. Rev. Phys. Chem. 59, 659683 (2008).

[20] K. Yasui, "Effect of volatile solutes on sonoluminescence", Journ. Chem. Phys. 116, 2945-2954 (2002).

[21] H. Cheung, A. Bhatnagar, and G. Jansen, "Sonochemical destruction of chlorinated hydrocarbons in diluted aqueous solutions", Environ. Sci. Technol. 25, 1510-1512 (1991).

[22] A. Kotronarou, G. Mills, and M. R. Hoffmann, "Ultrasonic irradiation of paranitrophenol in aqueous-solutions”, J. Phys. Chem. 95, 3630-3638 (1991).

[23] A. Kotronarou, G. Mills, and M. R. Hoffmann, "Decomposition of parathion in aqueous solution by ultrasonic irradiation”, Environ. Sci. Technol. 26, 1460 1462 (1992).

[24] P. R. Gogate, S. Mujumdar, and A. B. Pandit, "Sonochemical reactors for waste water treatment comparison using formic acid degradation as a model reaction", Adv. Environ. Res. 7, 283-299 (2003).

[25] V. G. Yechmenev, E. J. Blanchard, and A. H. Lambert, "Study of the influence of ultrasound on enzymatic treatment of cotton fabric", Text. Color. Chem. Amer. Dyestuff Rep. 1, 47-51 (1999).

[26] I. Z. Shirgaonkar, R. R. Lothe, and A. B. Pandit, "Comments on the mechanism of microbial cell disruption in high pressure and high speed devices", Biotechnol. Prog. 14, 657-660 (1998). 
[27] K. Yasui, T. Tuziuti, and Y. Iida, "Dependence of the characteristics of bubbles on types of sonochemical reactors", Ultrason. Sonochem. 12 (2005).

[28] D. Fernández Rivas, "Taming acoustic cavitation", Ph.D. thesis, University of Twente (2012).

[29] D. Fernández Rivas, L. Stricker, A. Zijlstra, H. Gardeniers, D. Lohse, and A. Prosperetti, "Ultrasound artificially nucleated bubbles and their sonochemical radical production", Ultrason. Sonochem. 20, 510-524 (2013).

[30] R. Mettin, P. Koch, D. Krefting, and W. Lauterborn, Advanced observation and modeling of an acoustic cavitation structure, in Nonlinear Acoustics at the Beginning of the 21st Century (O. V. Rudenko \& O. A. Sapozhnikov, MSU, Moscow) (2002).

[31] N. Bremond, M. Arora, C. D. Ohl, and D. Lohse, "Controlled multibubble surface cavitation", Phys. Rev. Lett. 96, 224501 (2006).

[32] N. Bremond, M. Arora, S. M. Dammer, and D. Lohse, "Interaction of cavitation bubbles on a wall”, Phys. Fluids 18, 121505 (2006).

[33] D. Fernández Rivas, A. Prosperetti, A. G. Zijlstra, D. Lohse, and H. J. G. E. Gardeniers, "Efficient sonochemistry through microbubbles generated with micromachined surfaces", Angew. Chem. Int. Ed. 49, 9699-9701 (2010).

[34] H. N. McMurray and B. P. Wilson, "Mechanistic and spatial study of ultrasonically induced luminol chemiluminescence", J. Phys. Chem. A 103, 3955-3962 (1999).

[35] R. Toegel, B. Gompf, R. Pecha, and D. Lohse, "Does water vapor prevent upscaling sonoluminescence?", Phys. Rev. Lett. 85, 3165-3168 (2000).

[36] R. Toegel, S. Hilgenfeldt, and D. Lohse, "Suppressing dissociation in sonoluminescing bubbles: The effect of excluded volume", Phys. Rev. Lett. 88, 034301 (2002).

[37] R. Toegel and D. Lohse, "Phase diagrams for sonoluminescing bubbles: A comparison between experiment and theory", J. Chem. Phys. 118, 1863 (2003).

[38] V. Kamath and A. Prosperetti, "Numerical integration methods in gas-bubble dynamics", J. Acoust. Soc. Am. 85, 1538 (1989-1548). 
[39] V. Kamath, A. Prosperetti, and F. N. Egolfopoulos, "A theoretical study of sonoluminescence”, J. Acoust. Soc. Am. 94, 248-260 (1993).

[40] J. A. Sethian, Level set methods and fast marching methods (Cambridge University Press, Cambridge) (1999).

[41] H. Gelderblom, A. G. Zijlstra, L. van Wijngaarden, and A. Prosperetti, "Oscillations of a gas pocket on a liquid-covered solid surface", Phys. Fluids 24, 122101 (2012). 


\section{2 \\ Thermal models for acoustically driven bubbles *}

The chemical production of radicals inside acoustically driven bubbles is determined by the local temperature inside the bubbles. Therefore, modelling of chemical reaction rates in bubbles requires an accurate evaluation of the temperature field and the heat exchange with the liquid. The aim of the present work is to compare a detailed PDE model in which the temperature field is spatially resolved with an ODE model in which the bubble contents are assumed to have a uniform average temperature and the heat exchanges are modelled by means of a boundary layer approximation. The two models show good agreement in the range of pressure amplitudes in which the bubble is spherically stable.

\subsection{Introduction}

In acoustically driven microbubbles extreme conditions of temperature and pressure can emerge, giving rise to chemical reactions, involving the gas inside the bubbles and the surrounding liquid ("sonochemistry", see e.g. refs. [1-6]). Even without

*Published as: [Laura Stricker, Andrea Prosperetti and Detlef Lohse, Validation of an approximate model for the thermal behavior in acoustically driven bubbles, J. Acoust. Soc. Am., 130(5) SI, 32433251, DOI: 10.1121/1.3626132, Part 2, 2011]. 
bubble-bubble interaction - i.e. in the case of a single isolated acoustically trapped bubble as in single-bubble sonoluminescence [7-11] - the fluid- and thermodynamics is still rather complex. Even if such a bubble remains spherical (i.e., is small enough and weakly enough driven), a complete description of the process still must take into account spatial pressure and temperature distribution both inside and outside the bubble, mass and heat diffusion, evaporation/condensation phenomena, change in transport parameters due to thermal and compositional changes of the mixture, inertial effects, as well as all chemical reactions of the unstable species in the bubble. Various models with an increasing degree of sophistication exist, see e.g. refs. [1220] and for a review ref. [11]. Clearly, the complexity of the process implies the need of simplifications when addressing practical problems, such as studying the chemical output.

In this paper we focus on the thermal behavior (achieved temperatures, heat fluxes in and out of the bubble), which governs the chemical reactions by Arrhenius' law. We want to compare the results from the numerical solutions of the advectiondiffusion PDE for the temperature field inside the bubble as described by Prosperetti and coworkers $[12,21]$ and others [22-24] with the results from a thermal boundary layer approximation of the full dynamics, which leads to the ODE model which has been developed in Twente [19, 20, 25]. As such ODE models are computationally much cheaper than solving the full PDEs of the gas flow inside the bubble, they are highly desirable in order to get a quick overview on the thermal conditions inside the bubble and the resulting chemical reactions. However, such simplifying ODE models must be verified against the results from the solution of the full PDEs. Such a verification is the aim of the present paper. From a sonochemical point of view, there is a temperature range where the radical production is optimal, regardless of the ambient pressure [26]. Therefore a precise determination of applicable limits of ODE-type approximations plays a crucial role in correct quantitative estimates of production/destruction of radicals.

ODE type approximations of the gas dynamics inside acoustically driven bubbles have a tradition, see the reviews [11, 27-29]. A first attempt was the adoption of the adiabatic approximation for the gas transformation with artificial increase of the liquid viscosity [30], in order to keep into account the energy loss and the subsequent thermal damping. However, this solution was found unsatisfactory, as it overestimated the damping of non linear oscillations, especially the first nonlinear resonant peak. A second attempt was to consider a gas transformation with a variable isoentropic index $\kappa(t)$, depending on the instantaneous Peclet number $P e(t)=|\dot{R}(t)| R_{0}^{2} / R(t) D_{g}(t) \quad[12,31,32]$, but also this model had strong limitations [33], as it was based on linear oscillation approximation and it could not include the 
effects of subharmonic components in the response. Nonetheless, it has succesfully been used in the context of single bubble sonoluminescence [13, 14, 18, 24, 34, 35], often even only with an effective polytropic exponent.

In the present work we use the ODE model based on the thermal boundary layer approximation of refs. [19, 20, 25]. It will be described in detail in section 8.2. Roughly speaking, this ODE model includes the Rayleigh-Plesset equation for the radial dynamics of the bubble, van der Waals law for the inner pressure, and the energy equation for the temperature, where the heat flux is estimated from a boundary layer approximation.

The PDE model, also described in detail in section 8.2, includes the RayleighPlesset equation, an ODE equation derived from momentum and continuity equations for the evolution of the inner pressure [32], and a PDE for the temperature, both inside and outside the bubble.

In both models we assumed a perfect gas inside the bubble, low Mach number regimes, spherical symmetry and thus shape stability. However, while the first two assumptions are generally realistic, the last two are strictly dependent on the specific parameter regime that are considered, as large and strongly driven gas bubbles become shape unstable. This shape instability is meanwhile well understood, even quantitatively [11,36-45]. Obviously, strictly speaking our results cannot be applied to shape unstable bubbles, as such bubbles decay to smaller ones, and for those cases special care has to be paid when comparing numerical results with experimental data.

\subsection{Summary of the models}

Both models studied in this work make use of the Rayleigh-Plesset equation to describe the radial dynamics of the bubble:

$$
\left(1-\frac{\dot{R}}{c_{L}}\right) R \ddot{R}+\frac{3}{2}\left(1-\frac{\dot{R}}{3 c_{L}}\right) \dot{R}^{2}=\frac{1}{\rho_{L}}\left(1+\frac{\dot{R}}{c_{L}}+\frac{R}{c_{L}} \frac{d}{d t}\right)\left[p_{B}-p_{A}\right] .
$$

Here time derivatives are denoted by a dot, $R$ is the bubble radius, $c_{L}$ and $\rho_{L}$ are the speed of sound and the density of the liquid, $p_{B}$ is the liquid pressure just outside the bubble surface and $p_{A}$ the ambient pressure in the liquid assumed to be given by

$$
p_{A}=p_{\infty}-P_{a} \cos \omega t,
$$

in which $p_{\infty}$ is the static pressure and $P_{a}$ the acoustic driving pressure. The period of the driving sound field is given by $\tau_{d}=2 \pi / \omega$. An explicit expression for $p_{B}$ results from normal stress balance at the bubble wall

$$
p=p_{B}+4 \mu_{L} \frac{\dot{R}}{R}+\frac{2 \sigma}{R},
$$


with $\mu_{L}$ the dynamic viscosity of the liquid and $\sigma$ the surface tension coefficient. The gas pressure in the bubble, $p$, may be regarded as spatially uniform as long as the Mach number of the bubble wall motion is not too large. In the left-hand side of (6.3) we have neglected the very small contributions due to the gas viscosity and the vapor pressure. As will be shown below, the temperature of the liquid at the bubble surface remains sufficiently low for this to be an excellent approximation.

The two models differ significantly in the way in which the pressure and temperature of the bubble contents are calculated. Here we provide a summary of the two formulations referring the reader to several papers for additional details and derivations $[20,44,46,47]$.

\subsubsection{PDE model for $\mathbf{T}(\mathbf{t})$}

In the detailed model of Refs. $[44,46]$ the gas pressure is found by solving

$$
\dot{p}=\frac{3}{R}\left(\left.(\gamma-1) \lambda \frac{\partial T}{\partial r}\right|_{R}-\gamma p \dot{R}\right),
$$

where $T$ is the gas temperature, $\gamma$ is the ratio of the gas specific heats, $\lambda=\lambda(T)$ is the gas thermal conductivity and $r$ the radial coordinate measured from the bubble center. The temperature distribution inside the bubble is given by

$$
\frac{\gamma}{\gamma-1} \frac{p}{T}\left[\frac{\partial T}{\partial t}+\frac{1}{\gamma p}\left((\gamma-1) \lambda \frac{\partial T}{\partial r}-\frac{1}{3} r \dot{p}\right) \frac{\partial T}{\partial r}\right]-\dot{p}=\nabla \cdot(\lambda \nabla T) .
$$

The derivation of this equation (see e.g. [46]) treats the gas as perfect and its pressure as spatially uniform.

The temperature in the liquid $T_{L}(r, t)$ is described by the standard constant-properties convection-diffusion equation neglecting compressibility effects and viscous dissipation:

$$
\rho_{L} c_{p, L}\left(\frac{\partial T_{L}}{\partial t}+\frac{R^{2} \dot{R}}{r^{2}} \frac{\partial T_{L}}{\partial r}\right)=\lambda_{L} \nabla^{2} T_{L}
$$

Here $c_{p, L}$ and $\lambda_{L}$ are the liquid specific heat and thermal conductivity.

At the bubble surface continuity of temperatures and heat fluxes are assumed:

$$
\begin{gathered}
T(R(t), t)=T_{L}(R(t), t) \\
\lambda \frac{\partial T}{\partial r}(R(t), t)=\lambda_{L} \frac{\partial T_{L}}{\partial r}(R(t), t)
\end{gathered}
$$

The gas temperature is assumed to be regular at the bubble center $r=0$ and the liquid temperature to remain undisturbed at the initial value $T_{\infty}$ far from the bubble. 


\subsubsection{ODE model for $\mathbf{T}(\mathbf{t})$}

This model $[19,20]$ makes no attempt to describe the spatial distribution of the gas temperature inside the bubble. Rather, it is formulated in terms of a volume-averaged value $\langle T\rangle$ determined by a global balance over the bubble volume expressing the first principle of thermodynamics:

$$
c_{\nu} m_{g}\langle\dot{T}\rangle=\dot{Q}-p \dot{V}
$$

where $m_{g}$ is the mass of gas inside the bubble, $c_{v}$ is the constant-volume specific heat of the gas and $V=\frac{4}{3} \pi R^{3}$ is the bubble volume. The net heat absorbed by the bubble per unit time is modelled as

$$
\dot{Q}=4 \pi R^{2} \lambda \frac{T_{\infty}-\langle T\rangle}{l_{t h}}
$$

with $l_{t h}$ an estimate of the thickness of the thermal boundary layer in the liquid. A correct prescription for this quantity is crucial for the physical realism of the model. The general properties of diffusion processes suggest

$$
l_{t h}=\sqrt{D \tau_{t h}}
$$

in which $D$ is the gas thermal diffusivity evaluated for $T=T_{\infty}$ and $\tau_{t h}$ an appropriate time scale which is chosen as $\tau_{t h}=R /|\dot{R}|$. A cutoff is required when $\dot{R}$ becomes too small. A consideration of the Fourier series solution of the diffusion equation in a bubble of constant radius (which is appropriate when $\dot{R}$ is small) leads to the estimate $l_{t h}=R / \pi$. In conclusion, the final expression for the estimate of the boundary layer is $[19,20]$

$$
l_{t h}=\min \left(\sqrt{\frac{R D}{|\dot{R}|}}, \frac{R}{\pi}\right)
$$

The gas pressure is obtained from a form of the van der Waals equation of state modified to take into account inertial effects of the gas:

$$
p=\frac{N_{t o t} k_{B}\langle T\rangle}{V-N_{t o t} B}-\frac{1}{2}\langle\rho\rangle R \ddot{R}
$$

where $\langle\rho\rangle$ is the volume-averaged gas density, $N_{\text {tot }}$ the total number of gas molecules, $k_{B}$ the Boltzmann constant and $B$ the molecular co-volume. 


\subsection{Numerical method}

The gas energy equation (5.14) of the detailed model is first reduced to a more manageable form by introducing the auxiliary variable

$$
\tilde{T}:=\frac{1}{\lambda\left(T_{\infty}\right)} \int_{T_{\infty}}^{T} \lambda\left(T^{\prime}\right) d T^{\prime} .
$$

After this step, the numerical solution of the model is carried out by first transforming it into a set of ordinary differential equations by a collocation procedure as described in ref. [12] and, in greater detail, in ref. [21]. We set

$$
\frac{\tilde{T}}{T_{\infty}} \approx \sum_{k=0}^{N} a_{k}(t) T_{2 k}(y),
$$

where $y=r / R(t)$ and the $T_{2 k}$ are even Chebyshev polynomials. The variable $y$ fixes the boundary at $y=1$ and the use of even polynomials guarantees a vanishing gradient at the bubble center $y=0$. The expansion (2.15) is substituted into the gas energy equation written in terms of $\tilde{T}$ and the result evaluated at the Gauss-Lobatto collocation points $y_{k}$

$$
y_{k}=\cos (\pi k / 2 N), \quad k=0,1, \ldots, N .
$$

Before subjecting the liquid energy equation to a similar treatment, the semiinfinite range $R(t) \leq r<\infty$ is mapped onto the finite range $1 \geq \xi \geq 0$ by the coordinate transformation

$$
\frac{1}{\xi}=1+\frac{r / R(t)-1}{l} .
$$

The length $l$ is a measure of the thermal diffusion length in the liquid and is taken as

$$
l=\ell \frac{\sqrt{D_{L} / \omega}}{R_{0}},
$$

with $D_{L}$ the liquid thermal diffusivity $D_{L}=\lambda_{L} / c_{p, L} \rho_{L}$ and $\ell$ a numerical constant. On the basis of the results described in ref. [21] a value of $\ell=20$ has been used in this work. After recasting the liquid energy equation (6.8) in terms of the new variable $\xi$, the liquid temperature is expanded in a truncated Chebyshev series similar to (2.15):

$$
\frac{T_{L}}{T_{\infty}} \approx \sum_{k=0}^{M} b_{k}(t) T_{2 k}(\xi),
$$


substituted into the equation and the result evaluated at the Gauss-Lobatto collocation points $\xi_{j}$

$$
\xi_{j}=\cos (\pi j / 2 M), \quad j=0,1, \ldots, M .
$$

Use of the even polynomials in (2.19) enforces the temperature condition at infinity in the form $\partial T_{L} / \partial r \rightarrow 0$ as $\xi \rightarrow 0$, i.e. as $r \rightarrow \infty$

The interface conditions (2.7) and (2.8), as written, are algebraic constraints among the unknown coefficients of the expansions (2.15) and (2.19). For numerical purposes it proves convenient to differentiate them with respect to time to find

$$
\begin{gathered}
\frac{\lambda\left(T_{\infty}\right)}{\lambda\left(T_{S}\right)} \sum_{k=0}^{N} \dot{a}_{k}=\sum_{j=0}^{M} \dot{b}_{j}, \\
\sum_{\ell=0}^{N} 4 \ell^{2} \dot{a}_{\ell}=-\frac{1}{l} \frac{\lambda_{L}}{\lambda\left(T_{\infty}\right)} \sum_{n=1}^{M} 4 n^{2} \dot{b}_{n},
\end{gathered}
$$

where $T_{S}$ is the bubble surface temperature.

These steps reduce the detailed model to a system of ordinary differential equations, the $N$ equations for $a_{k}$ arising from the collocation of the gas energy equation, the $M$ equations for $b_{k}$ arising from the collocation of the liquid energy equation, the two boundary conditions (2.21) and (2.22), the Rayleigh-Plesset radial equation (6.1) and the pressure equation (5.13). These equations (and notably those including the time derivatives of the temperature expansion coefficients) constitute a coupled linear system which is first solved for the derivatives by Gaussian elimination and then integrated in time by using the 6-th order Gear stiff solver implemented in the IMSL libraries [48].

In order to ascertain the accuracy of the time integration we monitored the ratios of the coefficients of the last to the first terms in the expansions, $\left|a_{N} / a_{1}\right|$ and $\left|b_{M} / b_{1}\right|$, checking that they remained smaller than $10^{-6}$ and $10^{-4}$, respectively, at all times. We found that 20 and 30 terms, respectively, for the gas and liquid temperature fields were sufficient to meet these condition.

To simplify the inverse mapping between the modified and original gas temperatures $\tilde{T}$ and $T$ the temperature dependence of the gas thermal conductivity was approximated by a linear relation

$$
\lambda=A+C T .
$$

The values $A=0.01165 \mathrm{~W} / \mathrm{mK}$ and $C=5.528 \times 10^{-5} \mathrm{~W} / \mathrm{mK}^{2}$ approximate the measured thermal conductivity of air over the range $200 \mathrm{~K} \leq T \leq 3000 \mathrm{~K}$ [12].

The other numerical values used in the simulations described in the next section were $c_{L}=1481 \mathrm{~m} / \mathrm{s}, \rho_{L}=1000 \mathrm{~kg} / \mathrm{m}^{3}, \mu_{L}=10^{-3} \mathrm{~kg} / \mathrm{ms}, \sigma=0.072 \mathrm{~N} / \mathrm{m}, c_{p, L}=$ 
$4182 \mathrm{~J} / \mathrm{kg} \mathrm{K}, \lambda_{L}=0.59 \mathrm{~W} / \mathrm{mK}$ and $B=5.1 \times 10^{-29} \mathrm{~m}^{3}$. These values are appropriate for an air-water system at normal temperature and pressure, $T_{\infty}=293.15 \mathrm{~K}$ and $p_{\infty}=$ $101.3 \mathrm{kPa}$.

\subsection{Results}

The results that follow refer to a sound frequency of $20 \mathrm{kHz}$, which is typical of much sonochemical work [49]. According to the theoretical results of ref. [29, 39, 41, 44] which later got experimentally confirmed [43, 45], at this frequency a 50 and a 100 $\mu$ m-radius bubble become spherically unstable at pressure amplitudes of the order of 30 and $15 \mathrm{kPa}$, respectively. At pressure amplitudes slightly above this threshold the bubble will develop shape oscillations superimposed on the volume mode. These oscillations lead to a break-up of the bubble at still higher amplitudes which it is difficult to quantify as they depend on various factors such as the perturbations induced by other bubbles, liquid motion and others. Even in the regime of weak shape oscillations a spherically symmetric model will capture the major effect responsible for the heating of the gas, namely the compression of the bubble. For this reason, and in order to bring out more clearly the differences between the two models, we will use pressure amplitudes of both $20 \mathrm{kPa}$ and $70 \mathrm{kPa}$.

The latter case $P_{a}=70 \mathrm{kPa}$ is shown in figure 2.1 for $R_{0}=130 \mu \mathrm{m}$ and $f=20 \mathrm{kHz}$. These conditions are close to resonance as for a $R_{0}=130 \mu \mathrm{m}$ bubble the linear natural frequency is approximately $24.4 \mathrm{kHz}$. The bubble executes strong volume pulsations with a maximum radius of about 3 times $R_{0}$, which corresponds to a maximum volume more than two orders of magnitude larger than the equilibrium volume. In contrast, for $P_{a}=20 \mathrm{kHz}$ only very gentle oscillations are observed (not shown). In both cases the differences between the ODE model and the PDE model are very small as can be seen in figure 2.1 for the $P_{a}=70 \mathrm{kPa}$ case (for the $P_{a}=20 \mathrm{kPa}$ case the differences are hardly detectable).

We now consider the effect of variations of the liquid temperature on the gas temperature and the radial dynamics of the bubble. The temperature $T_{S}$ of the liquid at the bubble surface was estimated in [12] as

$$
\frac{T_{s}-T_{\infty}}{T_{\text {center }}-T_{s}}=\sqrt{\frac{\lambda c_{p} \rho}{\lambda_{L} c_{p, L} \rho_{L}}}
$$

with $T_{\text {center }}$ the gas temperature at the bubble center, $c_{p}$ the gas specific heat at constant pressure and $\rho$ a measure of the gas density. On this basis the expected liquid temperature increase can be estimated to be small, but it is useful to go beyond estimates and determine quantitatively the actual importance of this effect. 


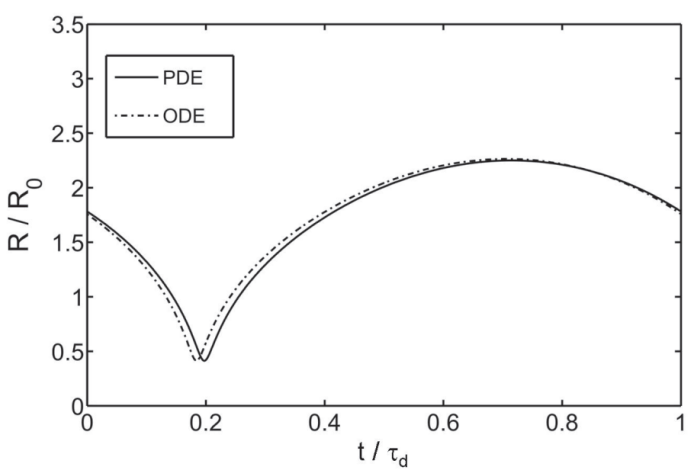

Figure 2.1: Comparison between the temporal evolution of the normalized radius during the steady oscillations of an air bubble with an equilibrium radius of $130 \mu \mathrm{m}$ driven at $70 \mathrm{kPa}$ and $20 \mathrm{kHz}$ as predicted by the detailed (solid line) and simplified models.

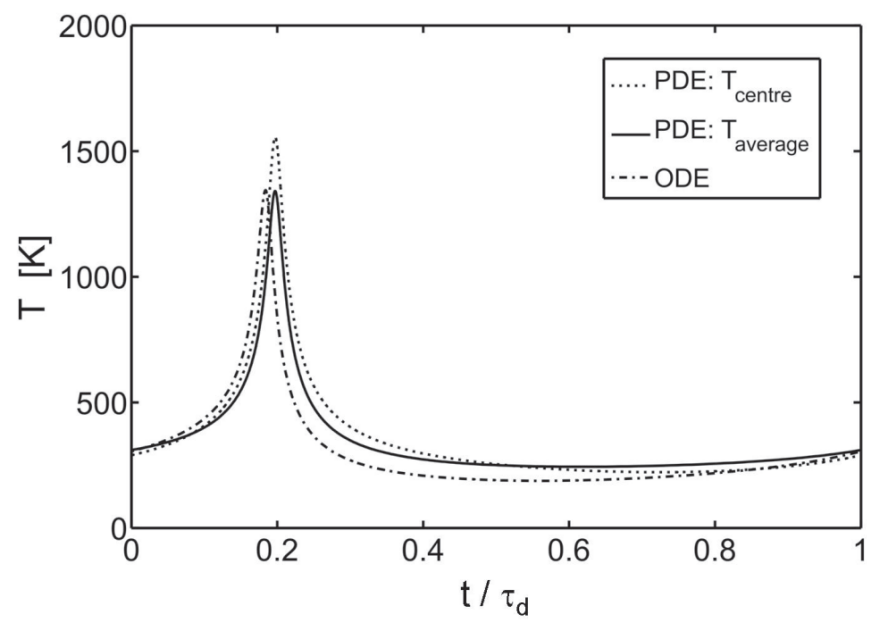

Figure 2.2: Comparison between the temporal evolutions of the average temperature according to the simplified model (dash-dot line) and the center (dotted line) and average (solid line) temperatures of the detailed model during steady oscillations for the same conditions as in figure 2.1

Figure $2.3 \mathrm{~b}$ shows the liquid temperature at the bubble surface as a function of time for the $130 \mu \mathrm{m}$-radius bubble driven at $20 \mathrm{kHz}$ with a pressure amplitude 
of $70 \mathrm{kPa}$. The temperature distribution in the liquid in correspondence of the peak surface temperature is shown in figure $2.3 \mathrm{~b}$. A space and time view of the temperature distribution in the gas and in the liquid in the course of a complete oscillation is provided in figure 2.4. It is seen that, even with oscillations of such relatively large amplitude, the maximum liquid temperature at the bubble surface increases by less than $15 \mathrm{~K}$ while the temperature at the core of the bubble becomes close to $1500 \mathrm{~K}$.

The effect of the liquid temperature on the gas temperature is demonstrated in figure 2.5 which compares the gas temperature distributions taken at the instant at which the peak values are predicted allowing or not allowing for variations of the liquid temperature. The detailed model provides the entire gas temperature distribution (solid line), which is seen to be very little affected by the neglect of the liquid temperature rise (dotted line). The simplified model only gives the average temperature without liquid temperature variations (dash-dot line), which is seen to be very close to the average temperatures calculated with the detailed model.

We conclude from these and other similar results not shown that temperature variations of the liquid have a negligible effect on the bubble gas temperature. This result is in line with the estimate (2.24) and the earlier results of ref. [12]. On this basis, in order to save computational time, in all the simulations described in the remainder of this paper we have kept the interface liquid temperature at the undisturbed value $T_{\infty}$. Correspondingly, we have replaced (2.21) by the simpler condition

$$
\sum_{k=0}^{N} \dot{a}_{k}=0 .
$$

An overall impression of how the two models compare can be obtained from figure 2.6 where the normalized maximum radius during steady oscillations is shown as a function of the equilibrium radius $R_{0}$ for driving pressure amplitudes of 20, 50 and $70 \mathrm{kPa}$; the sound frequency is $20 \mathrm{kHz}$ as before. As already noted, the spherical shape is expected to be unstable at $70 \mathrm{kPa}$, but we consider this value of the pressure amplitude to bring into clearer evidence the differences between the two models.

As could be expected, the main differences are localized around the linear and nonlinear resonance peaks and are seen to grow with the driving amplitude. In general it is observed that, as $R_{0}$ increases, the transition to a large-amplitude regime (signalled by the vertical or nearly vertical line; see e.g. ref. [30] for an explanation of the nature of this transition) occurs slightly earlier in the detailed model than in the simplified one. As a consequence, the maximum amplitude reached by the detailed model is slightly higher but the difference remains small for the pressure amplitudes studied.

For sonochemical applications, a key aspect of the phenomenon of bubble oscillations is the gas temperature. Figure 2.7 shows the maximum value of this quantity 


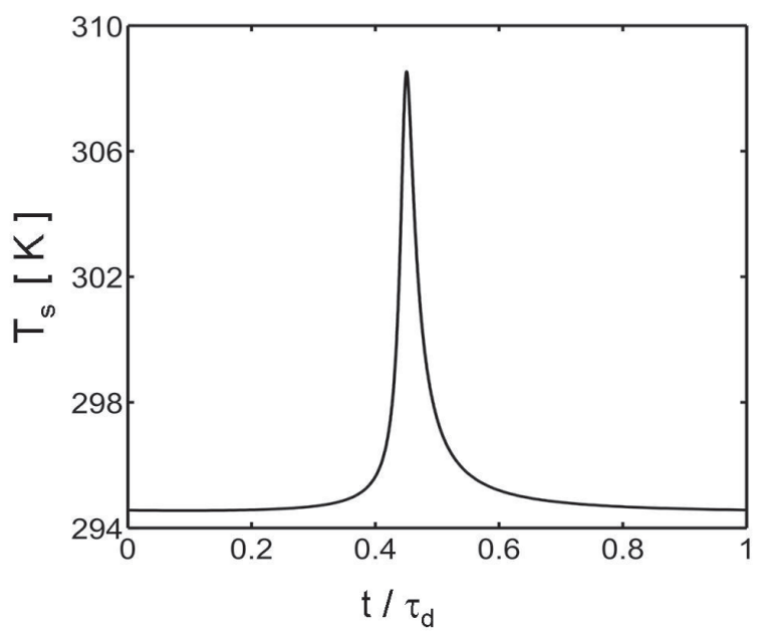

(a)

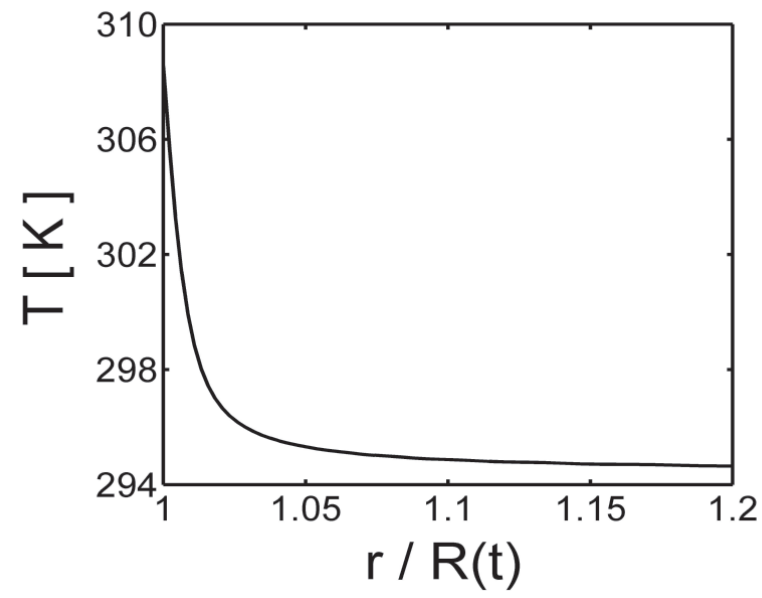

(b)

Figure 2.3: (a) Liquid temperature at the bubble surface during the steady oscillations of an air bubble in water with an equilibrium radius of $130 \mu \mathrm{m}$ driven at $20 \mathrm{kHz}$ by a sound field with a pressure amplitude of $70 \mathrm{kPa} ; \tau_{d}$ is the period of the sound field. (b) Liquid temperature distribution at the instant $t / \tau_{d}=0.45$ at which the bubble wall of the previous figure reaches its maximum temperature. 


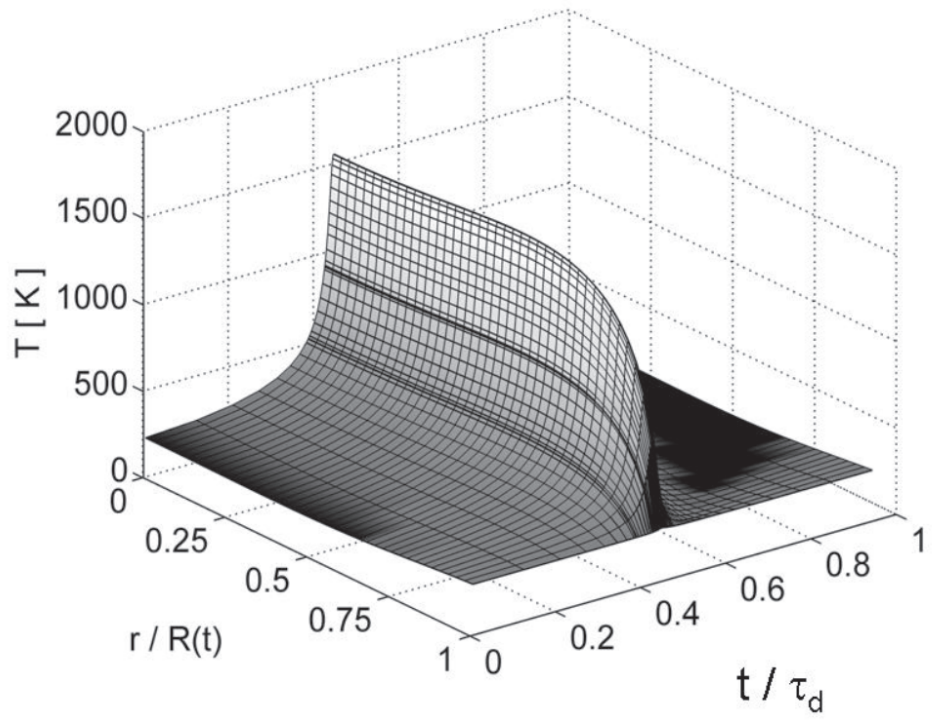

(a)

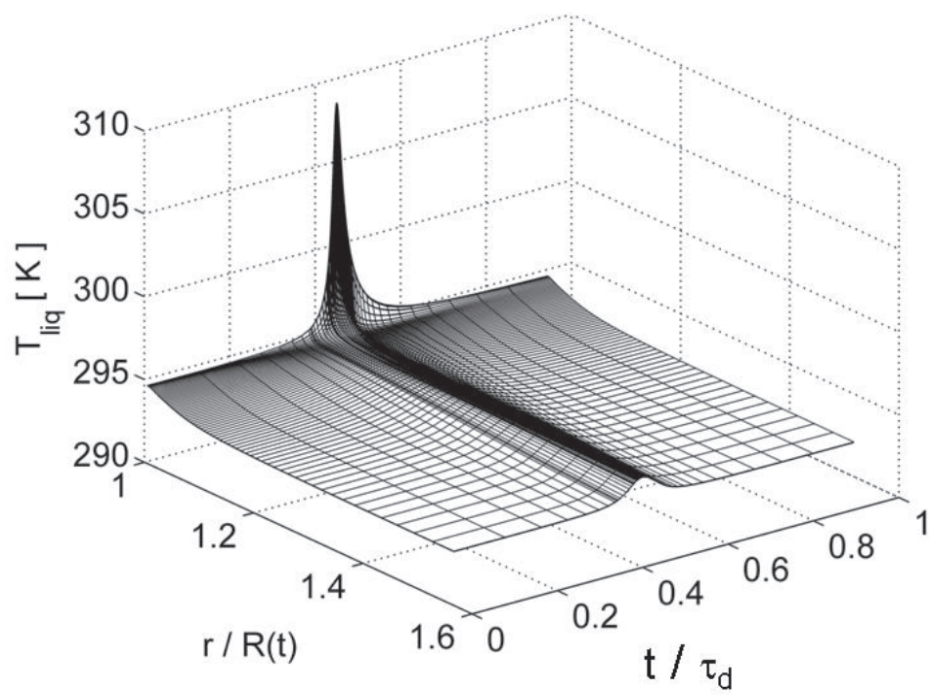

(b)

Figure 2.4: Temporal and spatial evolution of temperature inside (a) and outside (b) a steadily oscillating $130 \mu \mathrm{m}$ air bubble in water driven at $20 \mathrm{kHz}$ by a sound field with a pressure amplitude of $70 \mathrm{kPa}$. 


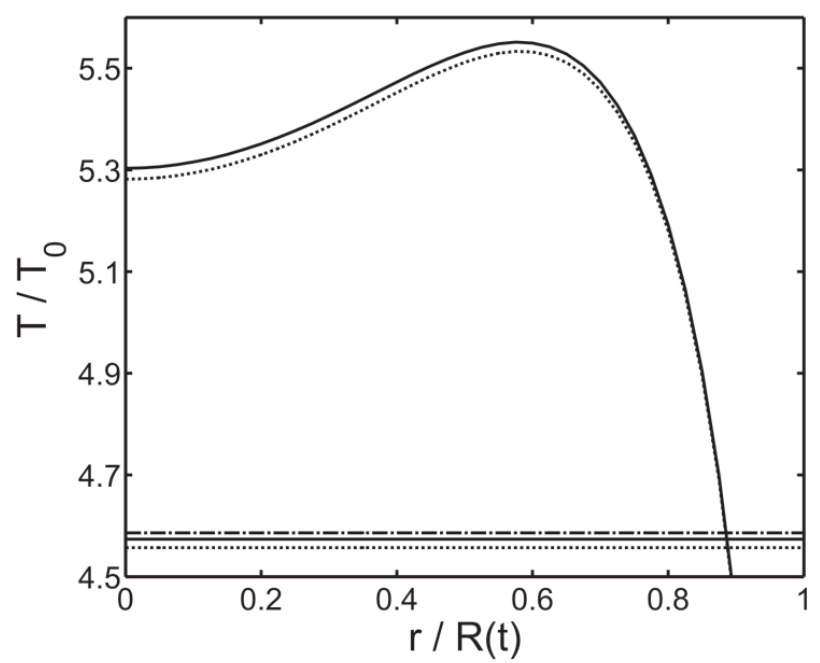

Figure 2.5: Gas temperature distribution with a fixed (solid line) and a variable (dotted line) liquid temperature according to the detailed model. The dash-dot line shows the (average) temperature according to the simplified model and the horizontal solid and dotted lies are the average values of the detailed model. The temperatures are shown at the instants at which the peak value is reached in each case.

as a function of $R_{0}$ for the same conditions as in figure 2.1. The center temperature for the detailed model is shown by the dotted line while the average temperature of the simplified model is indicated by the dash-dot line. The solid line is the volumeaveraged temperature predicted by the detailed model and calculated from

$$
\langle T\rangle=\frac{3}{R^{3}(t)} \int_{0}^{R} T(r, t) r^{2} d r .
$$

In correspondence with the larger maximum radius, the temperatures predicted by the detailed model are larger than that predicted by the simplified one, with a difference of a few hundred degrees attained in correspondence of slightly different radii near the main resonance at the largest driving amplitude. Just as in the case of the radius shown in figure 2.1, however, at the same value of the equilibrium radius the differences are not very large.

A more detailed view of the differences between the gas temperatures predicted by two models is shown in figure 2.2 for a $130 \mu$ m-radius bubble driven at $70 \mathrm{kPa}$ and $20 \mathrm{kHz}$. The average temperatures of the detailed (solid line) and simplified (dash-dot 


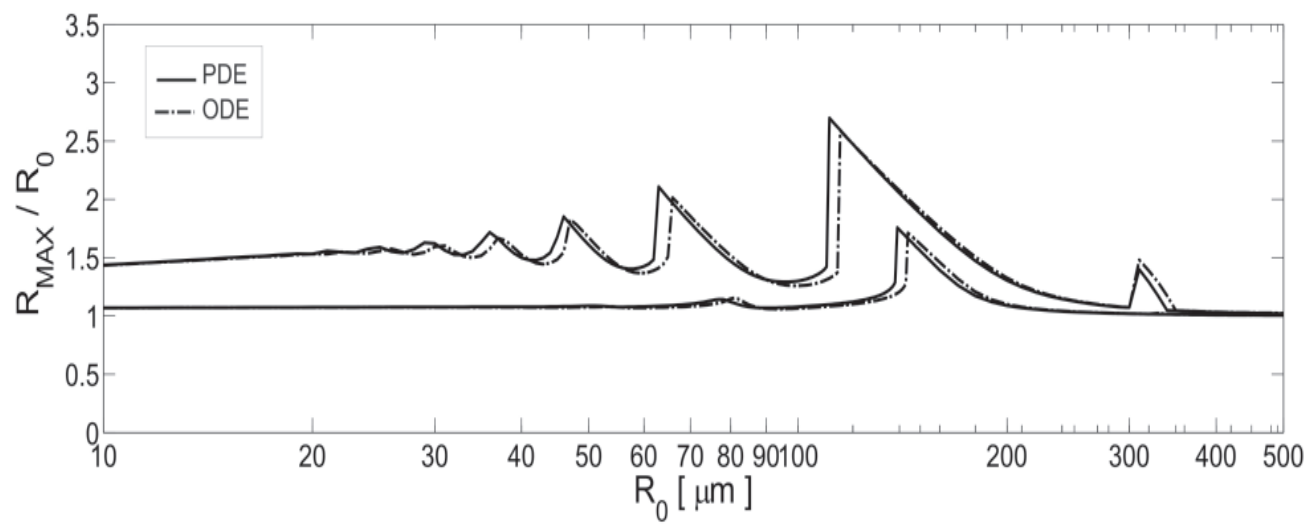

Figure 2.6: Normalized maximum radius during the steady oscillations of an air bubble in water driven at $20 \mathrm{kHz}$ as a function of the equilibrium radius $R_{0}$. The detailed and simplified model results are shown by the solid and dashed lines respectively. In ascending order, the driving pressure amplitudes are 20 and $70 \mathrm{kPa}$.

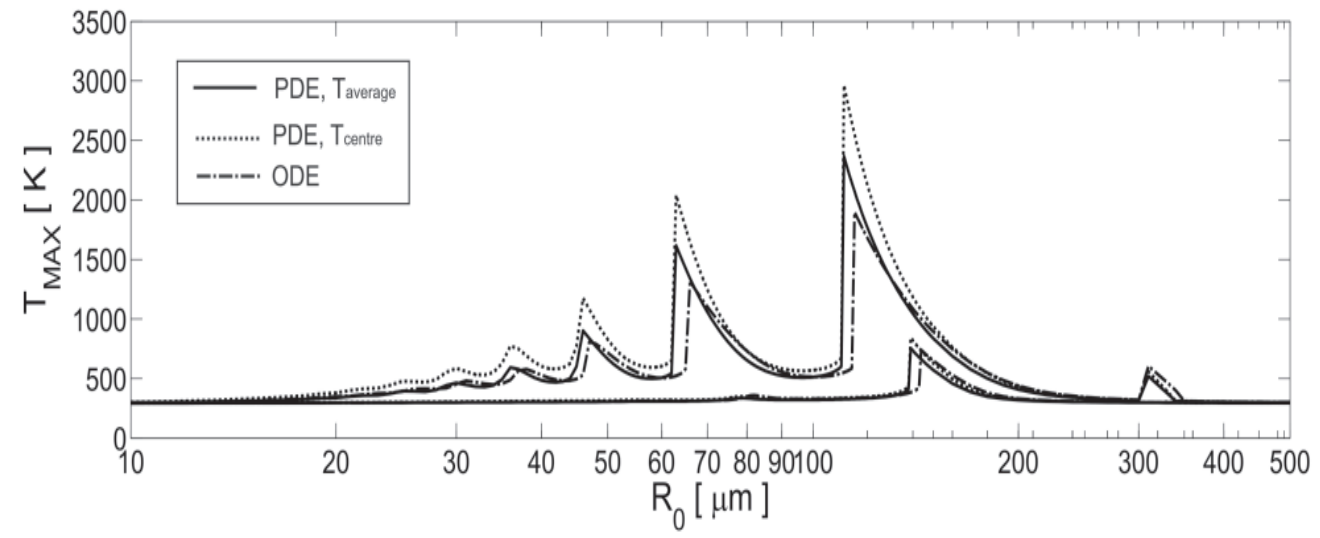

Figure 2.7: Gas temperature at the center of the bubble (dotted line), and mean temperatures according to the detailed (solid line) and simplified (dash-dot line) models during the steady oscillations of an air bubble in water driven at $20 \mathrm{kHz}$ as a function of the equilibrium bubble radius $R_{0}$. In ascending order, the driving pressure amplitudes are 20 and $70 \mathrm{kPa}$. 
line) models are nearly identical, while the center temperature of the detailed model peaks at a slightly higher value for a very short amount of time.

Figure 2.9 shows the normalized maximum and minimum temperature distributions inside bubbles with equilibrium radii of 30,130 and $305 \mu \mathrm{m}$ driven at $70 \mathrm{kPa}$ and $20 \mathrm{kHz}$. The solid and dotted lines are the local and average temperatures of the detailed model while the dash-dot lines are the average temperatures of the simplified model. The upper three lines refer to the instants at which the maximum average temperatures are reached in each model, and the lower three lines to the instants at which the minimum average temperatures are attained. The gas temperature distribution inside the largest bubble is approximately uniform except for a boundary layer near the wall. The temperature in the smallest bubble, on the other hand, exhibits a significant variation throughout the bubble volume. In this case the mean temperatures are very close, but the detailed distribution shows that this result comes about because the temperature in the inner region of the detailed model is offset by the relatively cool gas near the bubble wall. A good fraction of the gas is at a temperature about 20-30\% higher than the mean value. Given the at least approximate Arrhenius-law dependence of reaction rates, this difference in principle could have some observable effects in the sonochemical yield.

Related to the temperature distribution is the heat exchanged with the liquid which is given by eq. (4.4) in the simplified model and by

$$
Q=4 \pi R^{2}\left[\lambda \frac{\partial T}{\partial r}\right]_{r=R(t)}
$$

in the detailed model. The peak values of this quantity which, as defined, is positive when the transfer is directed from the liquid to the bubble, are shown in figure 2.10. The upper and lower diagrams show the heat lost and gained by the bubble respectively. A major qualitative difference between the two diagrams is the respective orders of magnitude. The heat lost by the bubble is more than one order of magnitude larger than that gained. This feature is at the root of the dominance of thermal energy losses over other dissipative mechanisms affecting the oscillations of bubbles below and around the resonance frequency (provided the radius is not too small as to make viscous losses significant). The heat losses predicted by the detailed model (solid line, upper diagram) are close to those of the simplified model except in a narrow radius range near the fundamental resonance for the highest driving pressure, where they are seen to be around $40 \%$ smaller. This is a large difference, but it occurs only during the brief instants in which the bubble is close to its minimum radius, as shown in figure 2.8. The differences among the incoming heat flow rates are much larger, particularly from the second harmonic region on up, but the absolute values are small. 
The distribution in time of the heat flow rate for the steady oscillations of a 130 $\mu \mathrm{m}$-radius bubble driven at $70 \mathrm{kPa}$ and $20 \mathrm{kHz}$ is shown in figure 2.8 . The solid line is the detailed model prediction and the dashed line that of the approximate model. The spike exhibited by the latter model near the point of maximum radius is an effect of the cutoff (2.12) applied when the boundary layer thickness becomes too large near the points of low radial velocity. This effect is highly localized in time and it is unlikely to have major consequences. In spite of the differences between the peak values shown in figure 2.10, one notices a substantial consistency between the two results over the complete course of an oscillation.

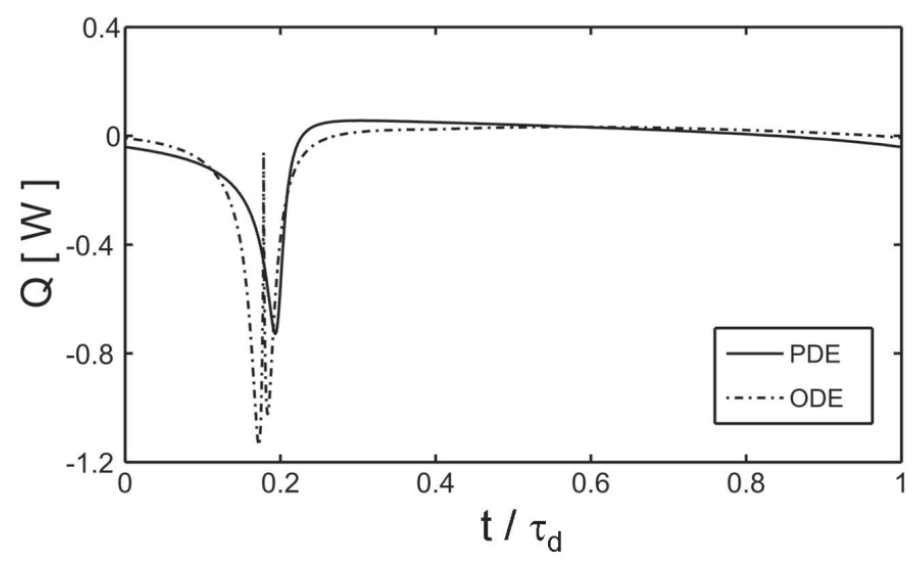

Figure 2.8: Heat flow rate into the bubble as a function of time during the steady oscillations of a $130 \mu \mathrm{m}$-radius air bubble in water with driven at $70 \mathrm{kPa}$ and $20 \mathrm{kHz}$ as predicted by the detailed (solid line) and simplified models. 


\subsection{Summary and conclusions}

In this paper we have compared two models of the forced oscillations of gas bubbles in liquids, devoting particular attention to the gas temperature in view of its importance for sonochemistry. The two models differ in their ability to capture details of the process. One accounts for the temperature distribution in the bubble and in the surrounding liquid, while the other one treats the bubble as a spatially homogeneous system. We have found that when the oscillation amplitude is moderate, namely at pressure amplitudes up to $70 \mathrm{kPa}$ or, for larger pressures, away from linear and nonlinear resonances, the two models are in very good agreement. Thus, in this parameter range, the simpler model can be used with confidence with the advantage of simpler programming and shorter execution times. For strong driving or near resonances we have found some differences, but it is then doubtful that bubbles would retain their integrity in view of their susceptibility to shape instabilities and break-up.

We have focused on the single driving frequency of $20 \mathrm{kHz}$ which is common in applications. At higher frequencies the picture would remain very similar provided radii are approximately shifted in inverse proportion to the frequency. Smaller bubbles, however, also tend to be more isothermal, with a consequent increase in energy loss. This feature is expected to reduce the difference between the two models at higher frequencies. The expectation is the opposite at lower frequencies, but larger bubbles are even more shape-unstable and, therefore, it is likely that neither model would be relevant except at rather low pressure amplitudes. 


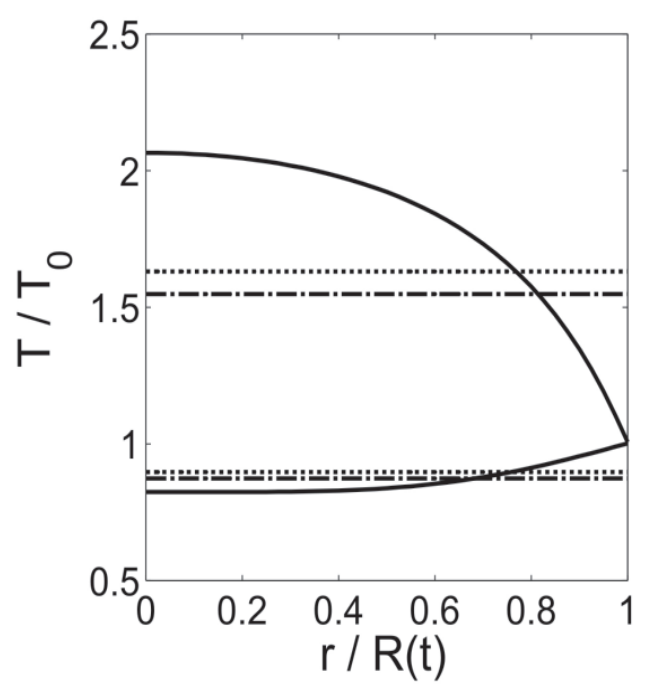

(a)

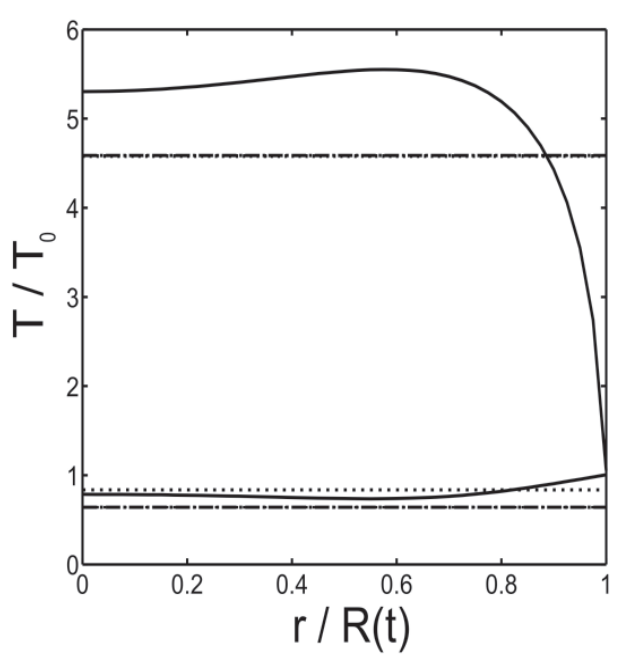

(b)

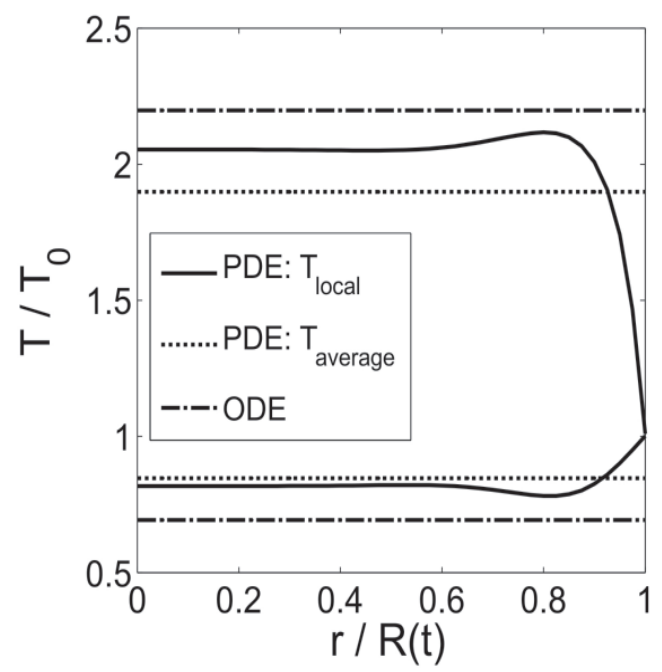

(c)

Figure 2.9: Normalized temperature distribution inside air bubbles in water with equilibrium radii of 30,130 and $305 \mu \mathrm{m}$ driven at $20 \mathrm{kHz}$ by a sound pressure amplitude of $70 \mathrm{kPa}$. In each figure the upper and lower groups of three lines refer to the instants at which the peak and minimum average temperatures are attained. The solid lines are the results of the detailed model, the horizontal dash-dot line the average temperature from the simplified model and the dotted horizontal lines the average temperature of the detailed model calculated from (2.26). 


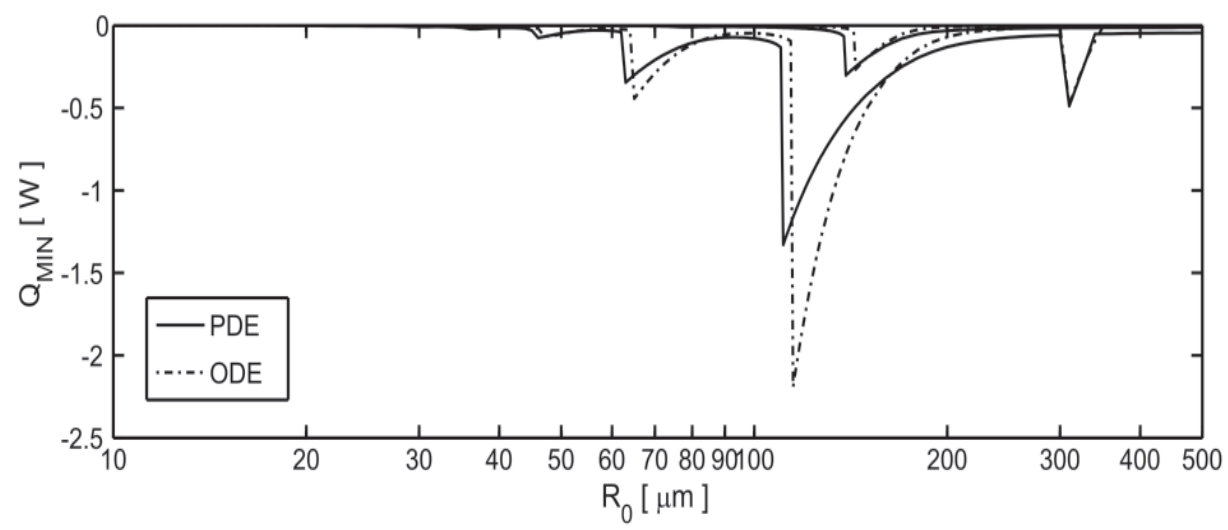

(a)

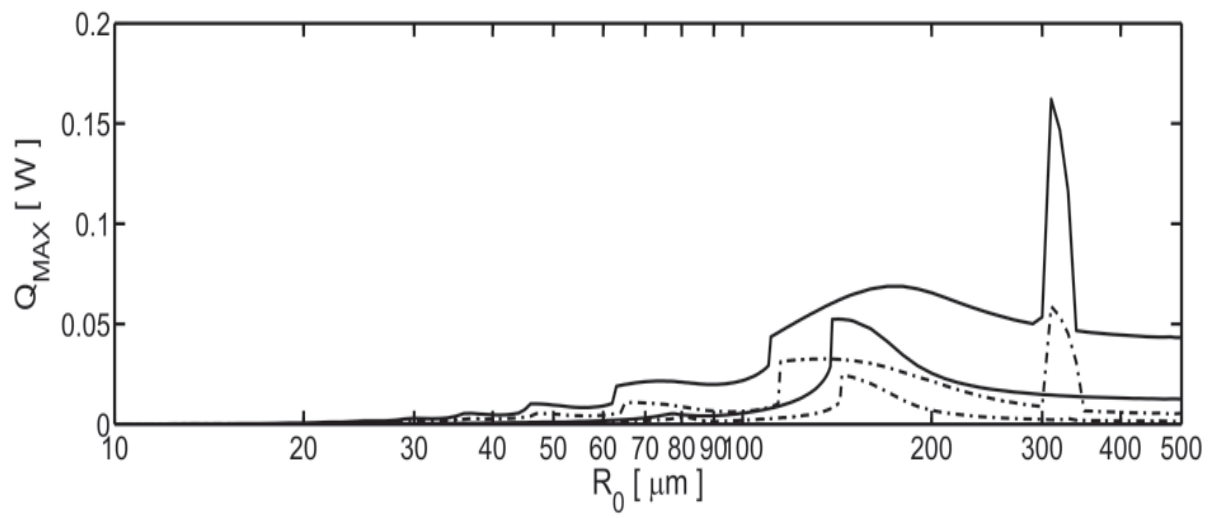

(b)

Figure 2.10: Peak values of the heat lost (upper figure) and gained by a steadily oscillating air bubble in water driven at $20 \mathrm{kHz}$ as a function of the equilibrium bubble radius $R_{0}$ according to the detailed (solid line) and simplified (dash-dot line) models. The sound pressure amplitudes are 20 and $70 \mathrm{kPa}$. 


\section{References}

[1] K. S. Suslick, "Sonochemistry”, Science 247, 1439-1445 (1990).

[2] K. S. Suslick, S. J. Doktycz, and E. B. Flint, "On the origin of sonoluminescence and sonochemistry”, Ultrasonics 28, 280-290 (1990).

[3] K. S. Suslick and G. J. Price, "Applications of ultrasound to materials chemistry", Ann. Rev. Mat. Sci. 29, 295-326 (1999).

[4] L. A. Crum, T. J. Mason, J. L. Reisse, and K. S. Suslick, eds., See the articles in Sonochemistry and Sonoluminescence (Kluwer Academic Publishers, Dordrecht) (1999).

[5] T. J. Mason and J. P. Lorimer, Applied sonochemistry, the uses of power ultrasound in chemistry and processing (Wiley-VCH, Weinheim) (2002).

[6] K. S. Suslick and D. J. Flannigan, "Inside a collapsing bubble: Sonoluminescence and the conditions during cavitation", Ann. Rev. Phys. Chem. 59, 659683 (2008).

[7] D. F. Gaitan, L. A. Crum, C. C. Church, and R. A. Roy, "Sonoluminescence and bubble dynamics for a single, stable, cavitation bubble", J. Acoust. Soc. Am. 91, 3166-3183 (1992).

[8] L. A. Crum, "Sonoluminescence”, Phys. Today 47, 22-29 (1994).

[9] B. P. Barber, R. A. Hiller, R. Löfstedt, S. J. Putterman, and K. R. Weninger, "Defining the unknowns of sonoluminescence", Phys. Rep. 281, 65-143 (1997).

[10] T. J. Matula, "Inertial cavitation and single-bubble sonoluminescence", Philos. Trans. R. Soc. London, Ser. A 357, 225-249 (1999).

[11] M. P. Brenner, S. Hilgenfeldt, and D. Lohse, "Single bubble sonoluminescence", Rev. Mod. Phys. 74, 425-484 (2002).

[12] V. Kamath, A. Prosperetti, and F. N. Egolfopoulos, "A theoretical study of sonoluminescence”, J. Acoust. Soc. Am. 94, 248-260 (1993).

[13] D. Lohse, M. P. Brenner, T. F. Dupont, S. Hilgenfeldt, and B. Johnston, "Sonoluminescing air bubbles rectify argon”, Phys. Rev. Lett. 78, 1359-1362 (1997).

[14] K. Yasui, "Alternative model of single-bubble sonoluminescence", Phys. Rev. E 56, 6750-6760 (1997). 
[15] K. Yasui, "Chemical reactions in a sonoluminescing bubble", J. Phys. Soc. Jpn. 66, 2911-2920 (1997).

[16] W. C. Moss, D. A. Young, J. A. Harte, J. L. Levatin, B. F. Rozsnyai, G. B. Zimmerman, and I. H. Zimmerman, "Computed optical emissions from a sonoluminescing bubble”, Phys. Rev. E 59, 2986-2992 (1999).

[17] B. D. Storey and A. J. Szeri, "Water vapour, sonoluminescence and sonochemistry”, Proc. Roy. Soc. London A 456, 1685-1709 (2000).

[18] B. D. Storey and A. J. Szeri, "A reduced model of cavitation physics for use in sonochemistry", Proc. Roy. Soc. London A 457, 1685-1700 (2001).

[19] R. Toegel, S. Hilgenfeldt, and D. Lohse, "Suppressing dissociation in sonoluminescing bubbles: The effect of excluded volume", Phys. Rev. Lett. 88, 034301 (2002).

[20] R. Toegel and D. Lohse, "Phase diagrams for sonoluminescing bubbles: A comparison between experiment and theory", J. Chem. Phys. 118, 1863 (2003).

[21] V. Kamath and A. Prosperetti, "Numerical integration methods in gas-bubble dynamics", J. Acoust. Soc. Am. 85, 1538 (1989-1548).

[22] V. Q. Vuong and A. J. Szeri, "Sonoluminescence and diffusive transport", Phys. Fluids 8, 2354-2364 (1996).

[23] H. Y. Cheng, M.-C. Chu, P. T. Leung, and L. Yuan, "How important are shock waves to single-bubble sonoluminescence?", Phys. Rev. E 58, 2705-2708 (1998).

[24] H. Lin and A. J. Szeri, "Shock formation in the presence of entropy gradients", J. Fluid Mech. 431, 161-188 (2001).

[25] R. Toegel, B. Gompf, R. Pecha, and D. Lohse, "Does water vapor prevent upscaling sonoluminescence?”, Phys. Rev. Lett. 85, 3165-3168 (2000).

[26] K. Yasui, T. Tuziuti, Y. Iida, and H. Mitome, "Theoretical study of the ambientpressure dependence of sonochemical reactions", J. Chem. Phys. 119, 347-356 (2003).

[27] M. S. Plesset and A. Prosperetti, "Bubble dynamics and cavitation", Annu. Rev. Fluid Mech. 9, 145-185 (1977). 
[28] T. G. Leighton, The acoustic bubble (Academic Press, London) (1994).

[29] C. E. Brennen, Cavitation and Bubble Dynamics (Oxford University Press, Oxford) (1995).

[30] A. Prosperetti, "Nonlinear oscillations of gas bubbles in liquids: steady-state solutions", J. Acoust. Soc. Am. 56, 878-885 (1974).

[31] A. Prosperetti, "Thermal effects and damping mechanisms in the forced radial oscillations of gas bubbles in liquid", J. Acoust. Soc. Am 61, 17-27 (1977).

[32] A. Prosperetti, L. A. Crum, and K. W. Commander, "Nonlinear bubble dynamics", J. Acoust. Soc. Am. 83, 502-514 (1988).

[33] A. Prosperetti and Y. Hao, "Modelling of spherical gas bubble oscillations and sonoluminescence", Philos. Trans. R. Soc. London, Ser. A 357, 203-223 (1999).

[34] D. Lohse and S. Hilgenfeldt, "Inert gas accumulation in sonoluminescing bubbles”, J. Chem. Phys. 107, 6986-6997 (1997).

[35] S. Hilgenfeldt, S. Grossmann, and D. Lohse, "A simple explanation of light emission in sonoluminescence", Nature 398, 402-405 (1999).

[36] M. Plesset, "On the stability of fluid flows with spherical symmetry", J. Appl. Phys. 25, 96 (1954).

[37] A. Prosperetti, "Viscous effects on perturbed spherical flows", Quart. Appl. Math. 34, 339-350 (1977).

[38] M. P. Brenner, D. Lohse, and T. F. Dupont, "Bubble shape oscillations and the onset of sonoluminescence”, Phys. Rev. Lett. 75, 954-957 (1995).

[39] S. Hilgenfeldt, D. Lohse, and M. P. Brenner, "Phase diagrams for sonoluminescing bubbles", Phys. Fluids 8, 2808-2826 (1996).

[40] R. G. Holt and D. F. Gaitan, "Observation of stability boundaries in the parameter space of single bubble sonoluminescence", Phys. Rev. Lett. 77, 3791-3794 (1996).

[41] M. P. Brenner, T. F. Dupont, S. Hilgenfeldt, and D. Lohse, "Reply to comment by Putterman and Roberts, Phys. Rev. Lett. 80, 3666-3667 (1998)”, Phys. Rev. Lett. 80, 3668-3669 (1998). 
[42] E. H. Trinh, D. B. Thiessen, and R. G. Holt, "Driven and freely decaying nonlinear shape oscillations of drops and bubbles immersed in a liquid: Experimental results", J. Fluid Mech. 364, 253-272 (1998).

[43] D. F. Gaitan and R. G. Holt, "Experimental observations of bubble response and light intensity near the threshold for single bubble sonoluminescence in an air-water system”, Phys. Rev. E 59, 5495-5502 (1999).

[44] Y. Hao and A. Prosperetti, "The effect of viscosity on the spherical stability of oscillating gas bubbles", Phys. Fluids 11, 1309-1317 (1999).

[45] M. Versluis, D. E. Goerts, P. Palanchon, I. L. Heitman, S. M. van der Meer, B. Dollet, N. de Jong, and D. Lohse, "Microbubble shape oscillations excited through ultrasonic parametric driving”, Phys. Rev. E 82, 026321 (2010).

[46] A. Prosperetti, "The thermal behavior of oscillating gas bubbles", J. Fluid Mech. 222, 587 (1991).

[47] R. Toegel, S. Hilgenfeldt, and D. Lohse, "The effect of surfactants on single bubble sonoluminescence", Phys. Rev. Lett. 84, 2509-2512 (2000).

[48] Visual Numerics, "IMSL Numerical Libraries", http://www.vni.com/ products/imsl/ (2010).

[49] L. H. Thompson and L. K. Doraiswamy, "Sonochemistry: Science and engineering”, Ind. Eng. Chem. Res. 38, 1215-1249 (1999). 


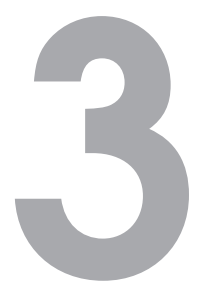

\section{Efficient sonochemical reactors $* \dagger$}

We describe the ejection of bubbles from air-filled pits micromachined on a silicon surface when exposed to ultrasound at a frequency of approximately $200 \mathrm{kHz}$. As the pressure amplitude is increased the bubbles ejected from the micropits tend to be larger and they interact in complex ways. With more than one pit, there is a threshold pressure beyond which the bubbles follow a trajectory parallel to the substrate surface and converge at the center point of the pit array. We have determined the size distribution of bubbles ejected from one, two and three pits, for three different pressure amplitudes and correlated them with sonochemical $\mathrm{OH}$ radical production. Experimental evidence of shock wave emission from the bubble clusters, deformed bubble shapes and jetting events that might lead to surface erosion are presented. We describe numerical simulations of sonochemical conversion using the empirical bubble size distributions, and compare the calculated values with experimental results.

*Published as: [David Fernandez Rivas*, Laura Stricker*, Aaldert G. Zijlstra, Han J.G.E. Gardeniers, Detlef Lohse and Andrea Prosperetti, Ultrasound artificially nucleated bubbles and their sonochemical radical production, Ultrason. Sonochem., 20(1), 510-524, 2013]; *these authors contributed equally to the present work.

${ }^{\dagger}$ The experimental data present in this chapter are entirely due to David Fernández Rivas. 


\subsection{Introduction}

Sonochemistry, the use of ultrasound in chemistry and processing [1], is a very promising field with applications in e.g. nanomaterials synthesis, degradation of contaminants in water treatment and the food industry [2-4]. In a sonochemical reactor each bubble acts as a single reactor in itself [5, 6], but the spatial distribution of these bubbles is normally not homogeneous, forming filamentary patterns or clusters $[7,8]$. Establishing the correlation between the sonochemical yield and the size, location, and dynamics of the bubbles in these clusters, may provide the knowledge to improve the efficiency of sonochemical reactors.

There is a considerable amount of experimental and theoretical work on single bubbles $[5,9,10]$ but, for practical applications, where the interest normally resides in multibubble systems, it turns out to be very difficult to extract bubble sizes and spatial distributions $[11,12]$. So far only holographic or laser techniques were successful in providing such information while also valuable information was obtained from bubble dissolution measurement [7, 13-19]. No study combining bubble distribution, sonochemical conversion and corresponding numerical simulations has been published.

At the root of this problem lies the fact that cavitation and its inception are extremely complex and very difficult to control. Once the conditions for cavitation inception exist, many phenomena have an influence on the functioning of the sonochemical reactor: bubble-bubble interaction, coalescence, surfactants or impurities dissolved in the liquid altering the bubble population and liquid properties, recirculation of the liquid inside the reactor, energy losses due to viscous heating, degassing of the liquid, energy conversion losses (e.g., electrical-to-mechanical) among others [20-28]. In this work we present a continuation of our first efforts to increase the efficiency of sonochemical reactors $[29,30]$. The concept is based on small predefined crevices in which stabilized gas pockets remain entrapped when introduced into the liquid [31-34]. When exposed to ultrasound, these gas pockets continuously generate chemically active cavitation bubbles at their location. These bubbles form peculiar and stable clouds in the reactor that do not occur in the absence of the pits.

A major advantage of this method is that the location of the bubbles is stable, known a priori, and coincides with the region of high intensity ultrasound. This feature is in contrast with the usual sonoreactors where bubbles occur randomly over large volumes. The reproducible cavitation structures generated in our system enable us to study the relation between the bubble size distribution, number of bubbles, spatial distributions, and chemical production rates. The latter are determined using dosimetry of $\mathrm{OH}$ radicals while the former are obtained using a nanosecond flashphotography technique. In this paper, the acquired rates in terms of radical production 
per bubble per acoustic cycle are discussed. Additionally, calculations provide further insight into some of the observed phenomena.

\subsection{Materials and Methods}

\subsubsection{Silicon micromachining}

The bubbles were generated from the gas entrapped in pits with a diameter of $30 \mu \mathrm{m}$ and a depth of $10 \mu \mathrm{m}$ etched in square silicon chips with a side of $10 \mathrm{~mm}$. These substrates were micromachined under clean room conditions on double-side polished silicon wafers with (100) crystallographic orientation. The pits were etched by means of a plasma dry-etching machine (Adixen AMS 100 SE, Alcatel). Pits arranged in three different configurations were used: single pits, two pits separated by a distance of $1 \mathrm{~mm}$, and three pits arranged at the corners of an equilateral triangle with sides of $1 \mathrm{~mm}$ (see Fig. 3.1).

\subsubsection{Set-up for US experiments and imaging technique}

A sketch of the experimental arrangement is shown in Fig. 3.2.

The cavitation cell was a glass container of $25 \mathrm{~mm}$ outer diameter, $15 \mathrm{~mm}$ inner diameter and $6 \mathrm{~mm}$ depth. The thickness of the cell bottom was $6 \mathrm{~mm}$ and matched one quarter of the wavelength at the operation frequency of $200 \pm 5 \mathrm{kHz}$ generated by a piezo Ferroperm PZ27 $6 \mathrm{~mm}$ thick and $25 \mathrm{~mm}$ in diameter glued to the cell bottom. To prevent loss of water by evaporation and gas by acoustic degassing the cell was covered by a glass slide resting on a rubber ring.

Two types of experiments were conducted, the measurement of sonochemical reaction rates and the imaging of the bubbles and their size distribution. For the first

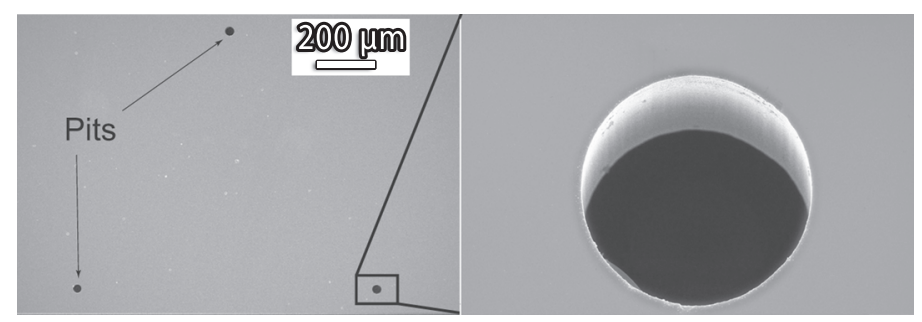

Figure 3.1: Pits micromachined on a silicon substrate by deep reactive ion etching. Top view (left) and a zoom-in perspective view (right). The diameter of the pit on the right is $30 \mu \mathrm{m}$. 


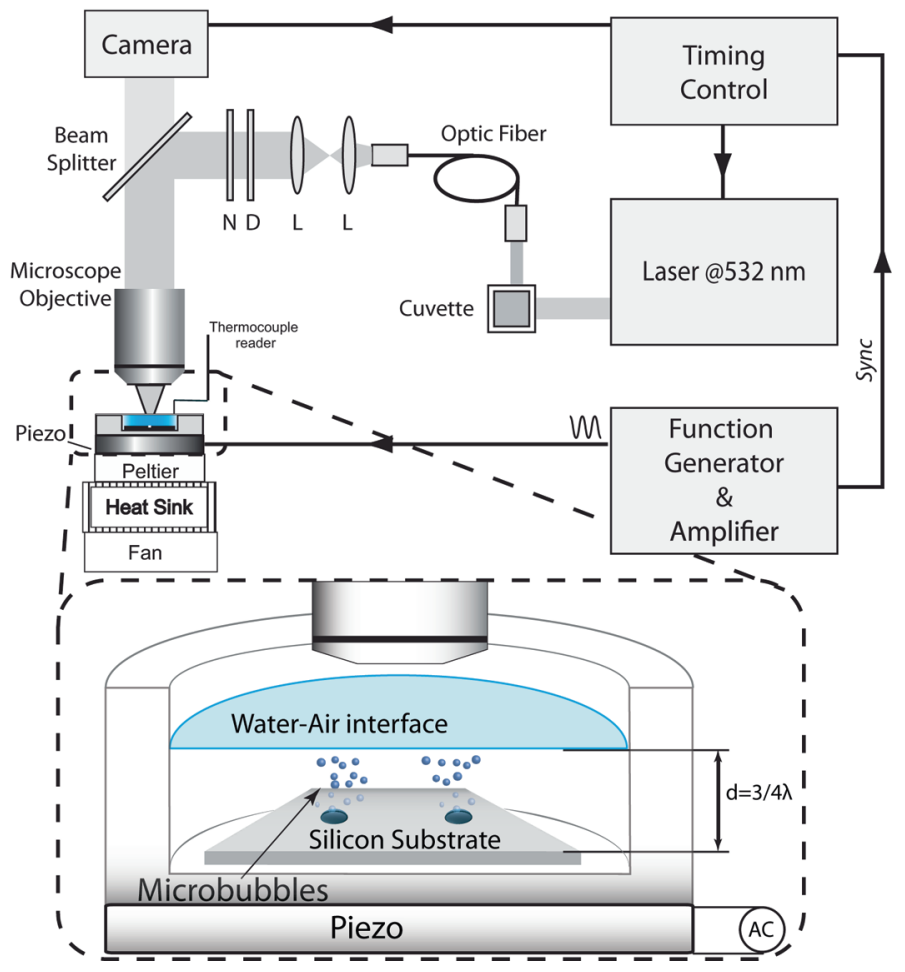

Figure 3.2: Experimental setup used for short exposure imaging and inset showing a detailed sketch of the cavitation cell.

type, the acoustic excitation was a sinusoidal signal provided by a Hewlett Packard 33120A arbitrary waveform generator amplified by means of a SONY TA-FB740R QS amplifier. The voltage and current provided to the piezo were measured with a Tektronix DPO 4034 oscilloscope and Tektronix current probe CTA2. The bottom surface of the piezo was kept in contact with a Peltier element (Marlow Industrial) to maintain the temperature at a constant value of $25^{\circ} \mathrm{C}$. At low and medium power the temperature increase during each experiment was measured to be no more than $1 \mathrm{~K}$, while in the high-power case the temperature increased no more than $3.8 \mathrm{~K}$, mainly during the first minute, and then remained constant. To study the radical production three different power settings were used with all three pit arrangements: low (0.074 $\mathrm{W})$, medium $(0.182 \mathrm{~W})$ and high $(0.629 \mathrm{~W})$.

The arrangement was similar for the bubble imaging experiments except for the use of a Krohn-Hite model 7602 wide-band amplifier and a Tabor Electronics Ltd. model WW1072 function generator. The different amplifiers resulted in differences 


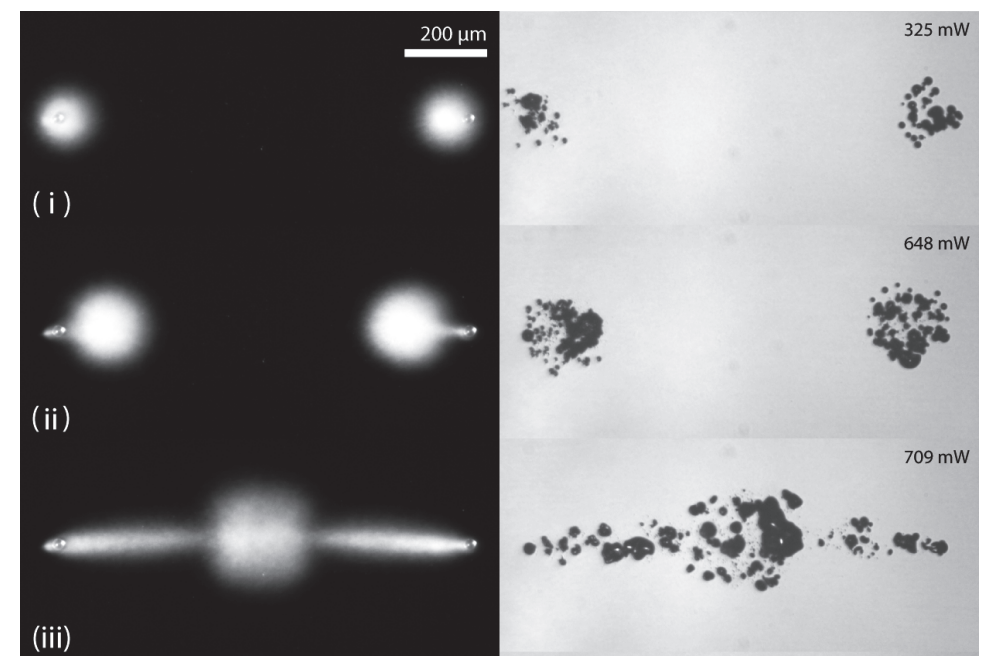

Figure 3.3: Bubble clouds originating from a two-pits substrate. Each row corresponds to a different acoustic amplitude increasing from top to bottom. The left column illustrates the time averaged extent of the cavitation cloud imaged at normal speed. The right column are single snapshots of $7 \mathrm{~ns}$ exposure time, in which individual bubbles can be distinguished at approximately maximum expansion.

in the electrical power supplied to the piezo element, which were $0.194 \mathrm{~W}$ (low), $0.654 \mathrm{~W}$ (medium) and $0.981 \mathrm{~W}$ (high). In this case space constraints prevented the use of the Peltier element.

Imaging was carried out with two cameras, one Photron model SA1.1 recording movies at normal speed ( $25 \mathrm{fps}$ ), the other one used for single snapshots was a Lumenera LM165 with a sensitive Sony EXview HAD CCD sensor. The Olympus microscope was equipped with two long working distance (WD) objectives, the LMPLFLN 10×, (WD $21 \mathrm{~mm} / \mathrm{NA}=0.25)$ and the SMPLFLN 20× (WD $25 \mathrm{~mm} / \mathrm{NA}=$ 0.25 ) both Olympus. Illumination was provided by a bright laser induced fluorescence pulse of $7 \mathrm{~ns}$ duration (full width at half maximum) which, amounting to about one thousandth of the acoustic period, enabled us to obtain high-resolution images without motion blur. The minimum radius detectable in the experiment was $\sim 0.5$ $\mu \mathrm{m}$. Additional details on this method can be found elsewhere [35]. Examples of the images obtained in this way are shown in Figures 3.3 and 3.4.

The purpose of these images was to obtain information on the number and sizes of the bubbles at various phases of the sound excitation. The difficulty to overcome was that the bubble density was high so that in many cases the images of different 
bubbles overlapped. To identify the individual bubbles it was therefore necessary to develop a special Matlab-based software using the so-called watershed segmentation algorithm [36]. After identification, the bubbles were counted and the image of each bubble was processed to extract the projected area and, with the assumption of a spherical shape, the equivalent diameter.

\subsubsection{Pressure amplitudes}

The quantity of interest for bubble oscillations is the acoustic pressure amplitude rather than the electrical power provided to the transducer. Unfortunately the conversion from one to the other is a notoriously difficult step. We attempted to measure the acoustic pressure amplitude by using a hydrophone (Onda HNR-500) with the tip positioned $65 \mu \mathrm{m}$ above the center point of the chip. The estimated error affecting these values according to the manufacturer is $20 \%$. The values found in this way were $165 \mathrm{kPa}$ for $0.074 \mathrm{~W}, 225 \mathrm{kPa}$ for $0.182 \mathrm{~W}$, and $350 \mathrm{kPa}$ for $0.629 \mathrm{~W}$. These values are uncertain due to several possible factors, electrical interference, the presence of waves on the surface of the liquid, bubble formation at the hydrophone tip and others. Furthermore, bubbles detune the cell and shield each other, therefore these values are provided only for reference purposes. A better estimate will be derived in Section 8.2 with the aid of numerical simulations.

\subsubsection{Radical dosimetry}

Conversion of terephthalic acid to 2-hydroxyterephthalic acid (HTA) was taken as a measure for the concentration of $\mathrm{OH}$ radicals. A calibration graph of fluorescence intensity as a function of HTA concentration was obtained following steps similar to those described in the literature [37]. Fluorescence was measured using a spectrofluorometer (TECAN Sapphire ${ }^{2 T M}$ ) with an excitation wavelength of 310 $\mathrm{nm}$. The fluorophore had a peak emission wavelength of $429 \mathrm{~nm}$, but the emission was scanned between 350 and $600 \mathrm{~nm}$. Following the method reported by Iida [38], the acid solution was prepared by mixing $2.0 \times 10^{-3} \mathrm{~mol} / \ell(0.332 \mathrm{~g})$ of terephthalic acid (Sigma-Aldrich) $5.0 \times 10^{-3} \mathrm{~mol} / \ell(0.200 \mathrm{~g})$ of $\mathrm{NaOH}$ and phosphate buffer $(\mathrm{pH} 7.4)$, prepared from $4.4 \times 10^{-3} \mathrm{~mol} / \ell(0.589 \mathrm{~g})$ of $\mathrm{KH}_{2} \mathrm{PO}_{4}$ and $\mathrm{Na}_{2} \mathrm{HPO}_{4}(7.0$ $\times 10^{-3} \mathrm{~mol} / \ell(0.981 \mathrm{~g})$ ) (all from Riedel - de Haën). The resulting solution was then made up to $1 \ell$ with milliQ water.

An amount of $300 \mu \ell$ of the solution measured with an Eppendorf micropipette was used in each experiment. At the end of the experimental run, the solution was pipetted out of the cell and stored in dark in sterilized vials (manufactured by Brand) for spectroscopic analysis. This step was conducted with $200 \mu \ell$ taken from the 
stored solution pipetted into the wells of a Corning Inc. well assay plate. The spectrophotometer settings for the analysis of the samples were: gain 40, height from the well, $9000 \mu \mathrm{m}$, integration time $100 \mu \mathrm{s}, 10$ reads per well.

Each experiment was repeated six to ten times and the mean concentration value was calculated. The largest standard deviation occurring for the case of three pits at maximum power, was about $30 \%$ of the average value.

Calculation of the radical formation rate was carried out according to:

$$
\frac{\Delta N_{\text {rad }}}{\Delta t}=\frac{\left(c_{\text {end }}-c_{\text {initial }}\right) N_{A} V}{\Delta t}
$$

in which $c_{\text {end }}$ and $c_{\text {initial }}=0$ are the final and initial concentrations of HTA in moles per volume, $N_{A}=6.02 \times 10^{23} \mathrm{~mol}^{-1}$ is Avogadro's number and $V$ is the liquid volume exposed to the US $(300 \mu \ell)$.

\subsubsection{Theoretical bubble dynamics and radical production calculation}

The model used to theoretically describe the bubble dynamics is based on an extended Rayleigh-Plesset-type equation, adopting the boundary layer approximation to estimate diffusive and thermal fluxes and assuming spherical symmetry for the bubble [39-42]. Temperature evolution over time is given by a global energy balance. The gas inside the bubble is taken as air with a van der Waals type equation of state. Changes in the transport parameters in accordance with compositional changes of the mixture are also included in the model. A driving frequency of $200 \mathrm{kHz}$ was taken, in agreement with experiments. Interaction between bubbles due to Bjerknes forces as well as coalescence effects were neglected.

\subsection{Results and Discussion}

\subsubsection{Description of the observed phenomena}

When the US is switched on, a continuous generation of bubbles is observed issuing from the gas stabilized in the pits on the silicon surface. These bubbles are likely caused by an instability of the liquid free surface at the pit mouth which undergoes large-amplitude oscillations under the action of the US. A similar process taking place at the surface of a free bubble has been described in the literature [7, 43, 44].

The resonance frequency of the pits is of the order of $150 \mathrm{kHz}$ and the radius of bubbles resonating at the applied frequency of $200 \mathrm{kHz}$ is about $15 \mu \mathrm{m}[45,46]$. Thus one would expect that, under the action of Bjerknes forces, bubbles smaller than this size would be repelled by the pits while larger ones would be attracted. At low 

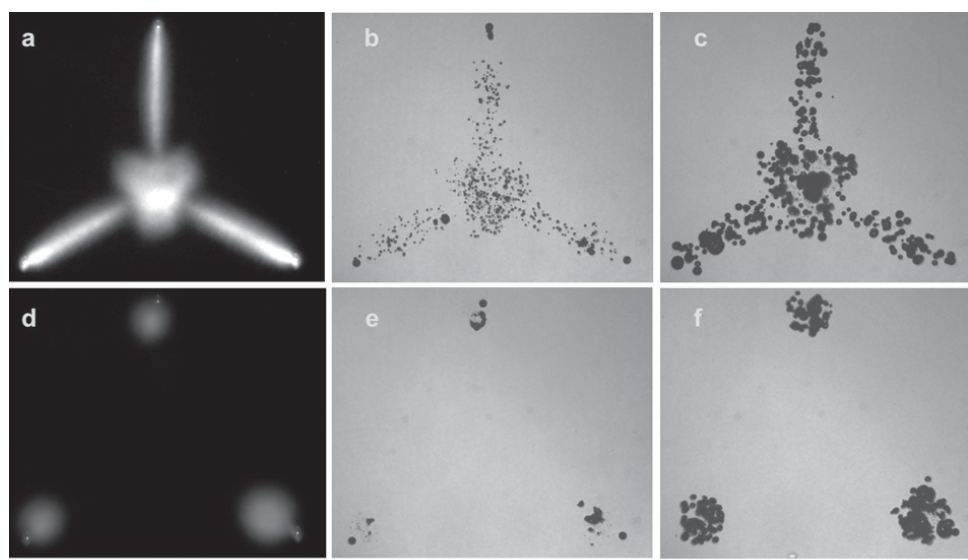

$1 \mathrm{~mm}$

Figure 3.4: Images of a substrate with three pits at different powers and at two selected points in the acoustic cycle. The upper row corresponds to high power $(0.981$ $\mathrm{W})$ and the lower row to low power $(0.194 \mathrm{~W})$. Visible with normal illumination conditions ( $\mathrm{a}$ and $\mathrm{d}$ ) and corresponding short exposure images: $\mathrm{b}$ and $\mathrm{e}$ in the compression; $\mathrm{c}$ and $\mathrm{f}$ in the expansion phase of the acoustic cycle.

power, the observed behavior is compatible with this expectation as shown in Figures 3.3 and 3.4. A few bubbles are observed at any given time. They move some distance away from the pit and survive a few cycles before disappearing [45].

As the power is increased, the ejected bubbles become more numerous forming clouds of increasingly larger size around the pits. At still higher powers, with more than one pit, the clouds appear to be attracted to each other until, at a fairly reproducible power level, they travel towards the central area of the pit array where they collect forming a single cloud (see Figures 3.3 and 3.4). As shown in Ref. [29], and as can be seen in the last frame of Fig. 3.8 below, these clouds when observed from the side resemble the conical clouds formed at the tip of sonotrodes [47, 48]. These structures are very different from the filamentary structures, sometimes called acoustic Lichtenberg figures [49], which are common in normal acoustic cavitation at high acoustic amplitudes.

The cloud is continually fed by new bubbles arriving from the pits under the action of the complex interplay of primary and secondary Bjerknes forces and microstreaming acting on the bubbles. The bubbles in the cloud are observed to break up seeding the liquid with their fragments which then grow into additional bubbles that join the cloud. The processes responsible for the accretion and loss of bubbles 

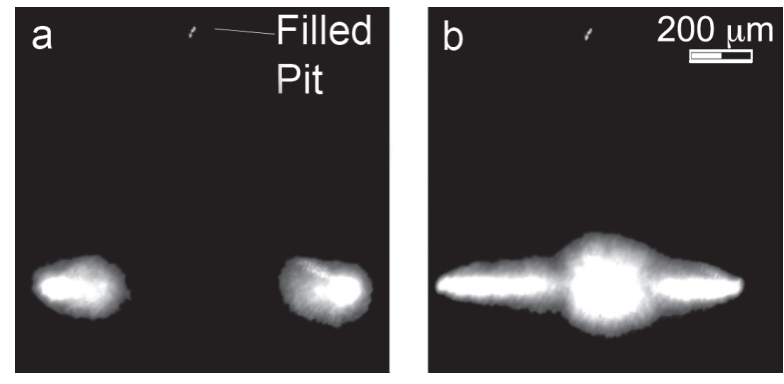

Figure 3.5: When a pit is filled with water in a three-pit substrate, the bubbles nucleated by the remaining active pits attain the same configuration as for a two-pit substrate: a, Medium power $(0.654 \mathrm{~W})$ and b, High power $(0.981)$.

eventually reach a steady state and the cloud is not observed to grow further.

The transition from clouds localized near the pits to a central cloud exhibits hysteresis, with the pressure amplitude necessary to return to separated clouds lower than that needed to bring them together.

The three-pits arrangement of Fig. 3.4 shows transition to a pattern similar to that of Fig. 3.3 for two pits when one of the three pits is rendered inactive (see Fig. 3.5). This observation suggests that liquid flow does not play a major role in the observed cloud attraction, which therefore must be mostly due to Bjerknes forces.

At high power a region of damaged silicon substrate forms near the pits within 5 minutes of exposure to the US (see Fig. 3.6). The shape of the individual damage sites coincides with the crystal planes of the crystalline structure of the silicon wafers. Damage to other parts of the chip and, in particular, under the large cloud forming over the center of the pit array, is much more modest and takes a significantly longer time. For more details see Ref. [30]

We have found that this localized acoustic bubble generation can be sustained for at least several hours if temperature is kept constant and gas loss is prevented by means of the glass slide mentioned before. Therefore we must conclude that the loss of gas associated to the bubble generation does not deactivate the pits, which is evidence of a process of rectified gas diffusion into the pits similar to the well-known process taking place with free bubbles.

The emission of shocks by collapsing bubbles is a known feature [50, 51]. An example in our case is shown in Fig. 3.7.

When bubbles are close to each other or to the solid surface, which preferentially occurs at higher power, they tend to deform and to interact. When bubbles are close enough, they develop liquid jets towards each other (see as an example [52]). Fig. 

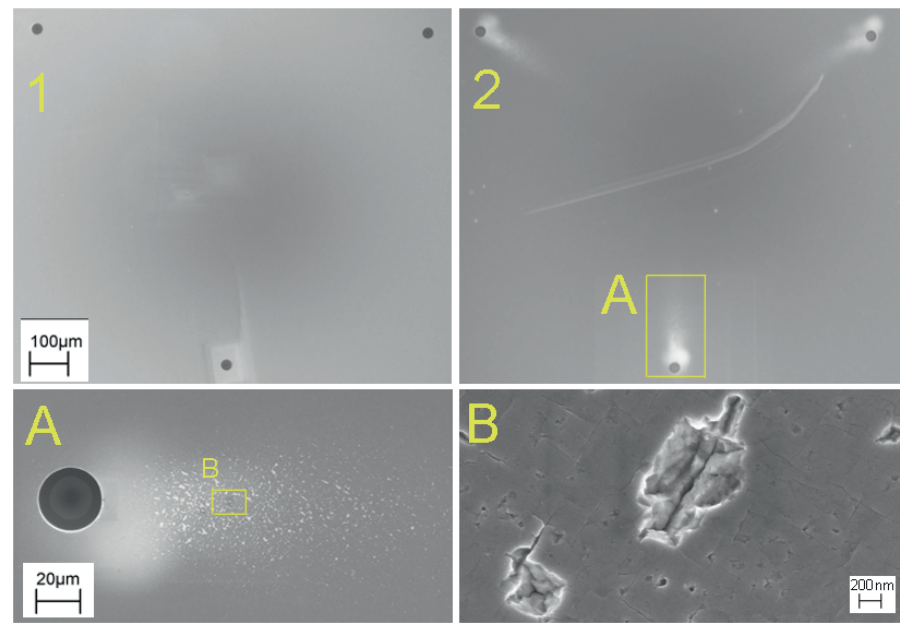

Figure 3.6: SEM image affording a comparison of a substrate before (1) and after (2) use in ultrasonic experiments. The damage pattern and zoom-in views of it, show that the eroded surface coincides with the $\{111\}$ silicon crystal planes.

3.8 shows several examples of such deformations and interactions.

In interpreting these images it must be kept in mind that bubbles smaller than resonance size expand during the acoustic compression phase, while bubbles bigger than resonance size contract. At high powers these latter bubbles may become unstable and split, generating the significantly smaller bubbles seen in the figure. The actual stability of these bubbles will also be influenced by their distance from the solid substrate and the proximity of other bubbles. Some of the smaller bubbles may also become unstable and fragment during collapse, but this would happen during the acoustic expansion phase.

We have estimated the translational velocity of typical bubbles on the basis of some preliminary experiments by imaging the acoustic field at $1.4 \mathrm{Mfps}$. We found typical velocities of about $10 \mathrm{~m} / \mathrm{s}$ and corresponding lifetimes of $100 \mu \mathrm{s}$, which is of the same order as that reported for other typical sonoreactors at similar frequencies [18] where bubbles had a lifetime of the order of $350 \mu \mathrm{s}$.

\subsubsection{Bubble size distribution}

As explained before, in order to characterize the bubble size distribution and its evolution in time over an acoustic period, we took snapshots of the cavitating region with an exposure of $7 \mathrm{~ns}$. The repetition time of the laser did not allow us to take consec- 

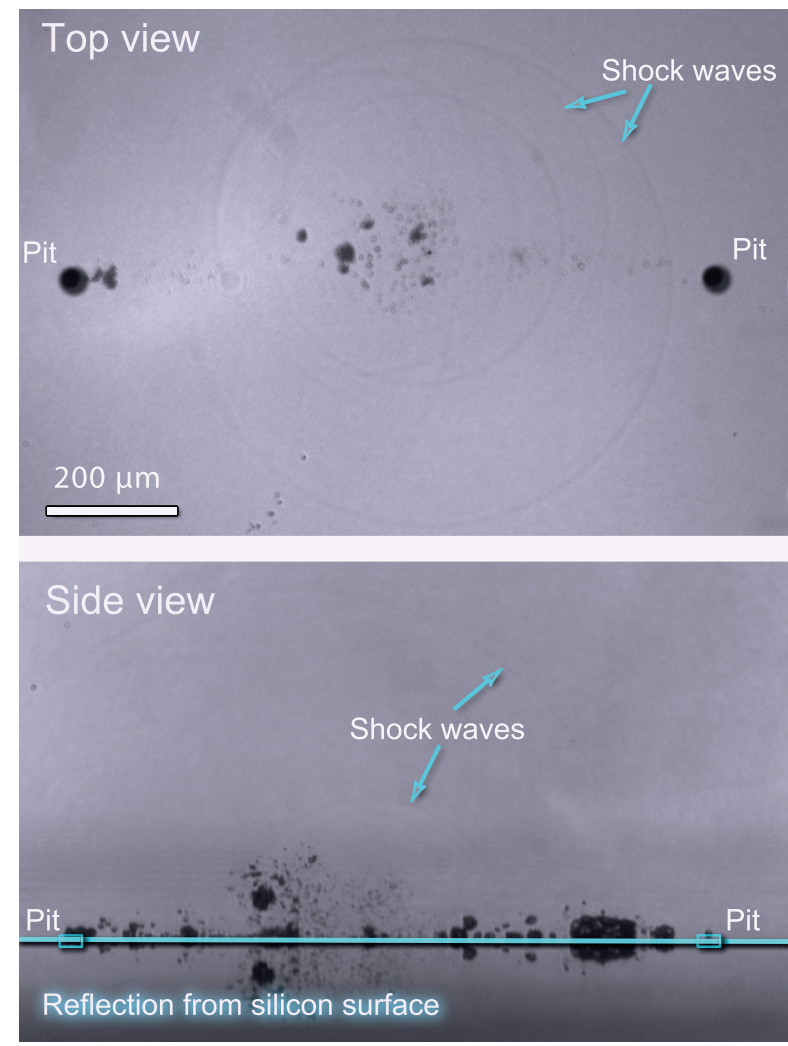

Figure 3.7: Shock waves emitted in the two-pit, high power situation $(0.981 \mathrm{~W})$. Several shock waves are observed in top and side views.

utive images faster than about 1 per second, i.e., every 200,000 acoustic cycles. The laser timing was set so as to generate a flash at a fixed phase of the sound field. We selected 10 phases and took 10 images per phase to improve the statistics.

As shown in Table 3.1, at the low power settings the number of bubbles in each frame was relatively small, typically 3-5 per pit and sometimes even less. Furthermore, the occasional overlaps of the bubble and pit images made it difficult to accurately count these bubbles. The measured low-power bubble populations, therefore, are not very accurate but are presented for reference as a lower bound; an example is provided in Fig. 3.9.

At the medium power settings the statistics improves since the bubbles tend to be more numerous, to grow larger and to move away from the pits. An example of a probability density function (PDF) for medium power is shown in Fig. 3.10. 


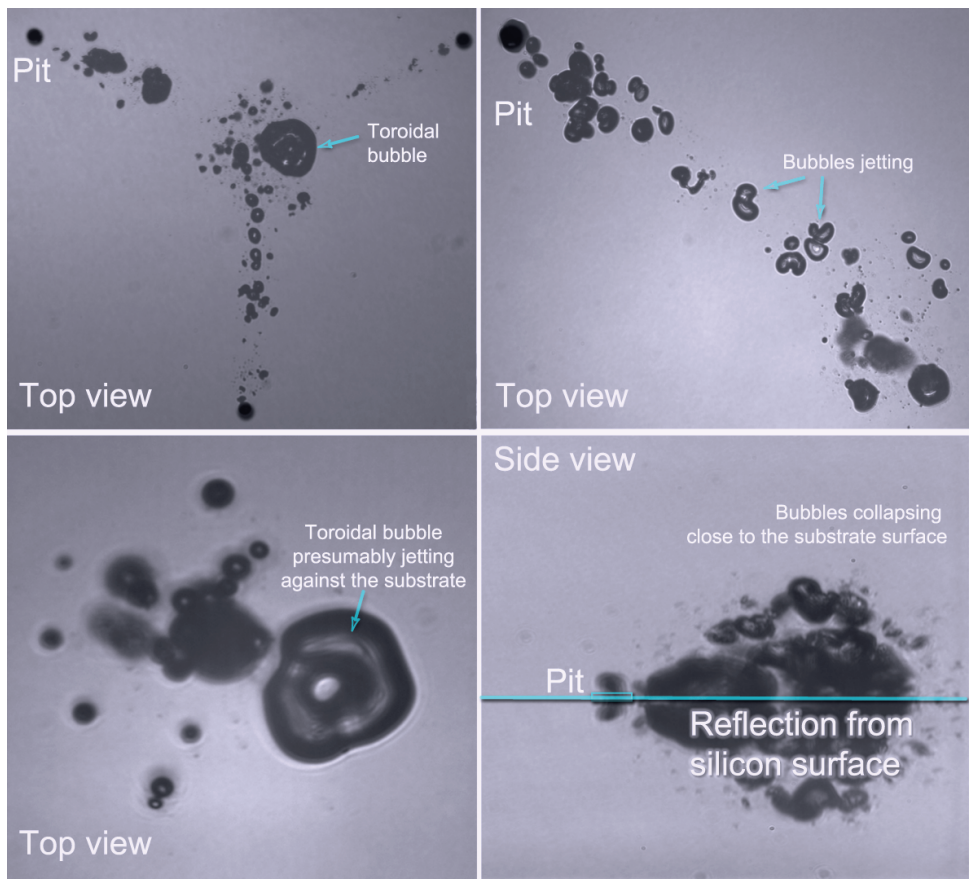

Figure 3.8: Deformed bubbles at high power settings $(0.981 \mathrm{~W})$. Note how bubbles tend to deform in all cases. When two bubbles are close, they develop liquid jets towards each other as in the upper right picture.

At high power $(0.981 \mathrm{~W})$ and one-pit configuration the expansion and collapse of the ejected bubble are very dramatic and their shape is highly deformed. The equivalent radii, therefore, are less representative of the actual size of a corresponding spherical bubble. The number of bubbles can also be under-estimated since some large bubbles overlap and the post-processing code can consider two or a blob of several bubbles as a single one. Nevertheless it provides a qualitative measure of their number. A typical PDF for high power is depicted in Fig. 3.11.

The bubble size distribution for a certain acoustic phase is the distribution of equivalent radii of the total number of bubbles over the 10 frames taken at that phase. The average radius is obtained by averaging the radii of all the bubbles in the 10 frames and the most probable radius is that with most occurrences at that phase. For example, a bubble size distribution such as that in Fig. 3.11 for $t / T=0$ is right-skewed i.e. more frequent smaller equivalent radii (R). The most probable radius is $\sim 2 \mu \mathrm{m}$, but the average radius is larger, as the distribution includes a range of radii up to 35 


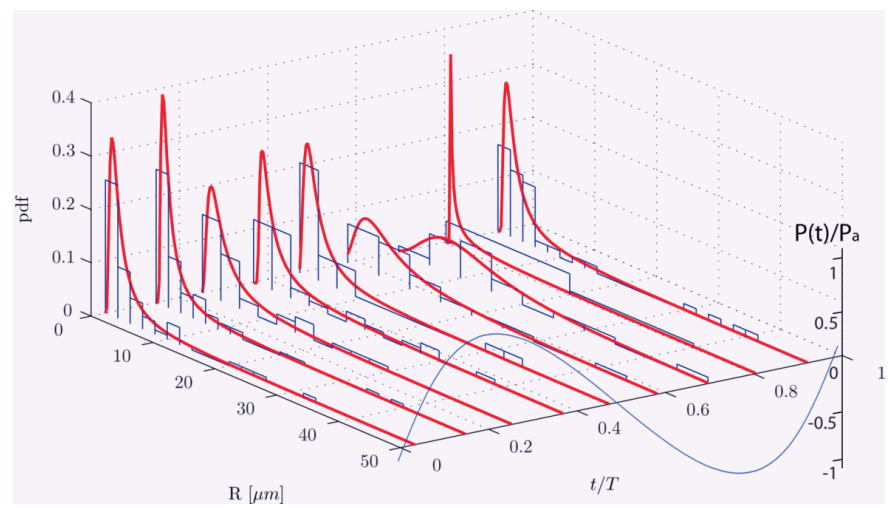

Figure 3.9: Bubble size distribution histograms at a power of $0.194 \mathrm{~W}$ for one pit. The axis to the extreme right represents the normalized pressure for the acoustic cycle.

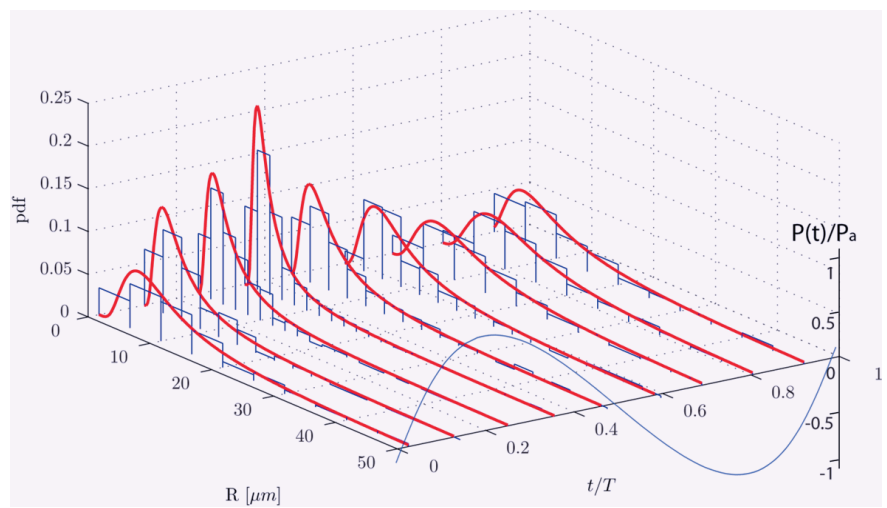

Figure 3.10: Bubble size distribution histograms for a power of $0.654 \mathrm{~W}$ for two pits. The axis to the extreme right represents the normalized pressure for the acoustic cycle. 


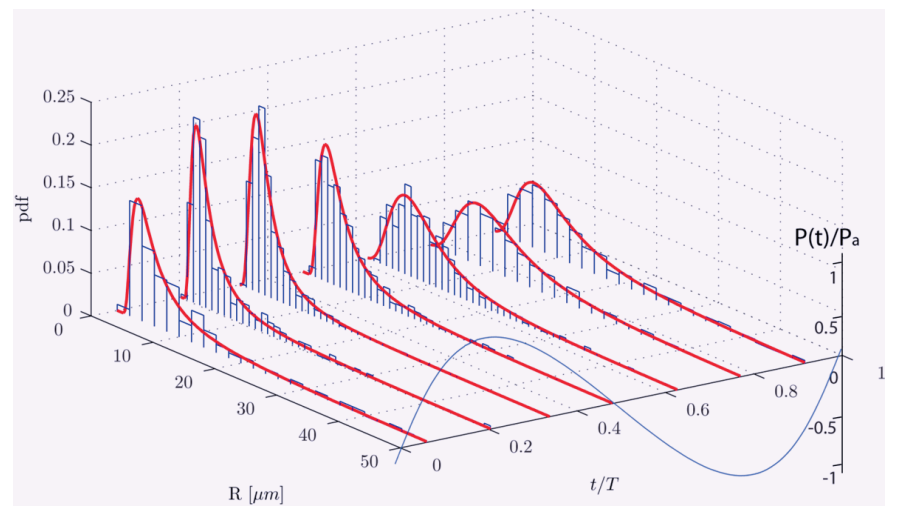

Figure 3.11: Bubble size distribution histograms at a power of $0.981 \mathrm{~W}$ for three pits. The axis to the extreme right represents the normalized pressure for the acoustic cycle.

$\mu \mathrm{m}$. See Fig. 3.12 for more details.

Table 3.1 presents a compilation of the experimental data on several measures of bubble size and average number per cycle. As noted above, the radius of resonant bubbles at the experimental frequency is about $15 \mu \mathrm{m}$. The measured average bubble radius is of this order ( 8 to $16 \mu \mathrm{m}$ ) and the most probable radius is between 2 and 5 $\mu \mathrm{m}$. Figure 3.12 shows an example of the evolution of the most probable and average radii, together with the number of bubbles during one acoustic cycle at $0.981 \mathrm{~W}$ for three pits. It is seen in the bottom panel of this figure that the number of bubbles peaks shortly after the maximum pressure. This feature may reflect the expansion of the smaller bubbles which are below the optical resolution of our apparatus during the rest of the acoustic cycle.

The average number of bubbles instantaneously present is shown in Fig. 3.13. These data were obtained by summing the number of all bubbles visible in all frames (10 frames each for 10 phases) and dividing by a hundred. For the one-pit configuration, as the power is increased the number of bubbles increases as would be expected. The results shown for low and medium power correspond to isolated bubble clouds while for two and three pits at high power the clouds have merged as explained before. When this happens, the observed bubble number does not increase, possibly due to bubble-bubble interactions and the finite amount of gas available [53-55]. 

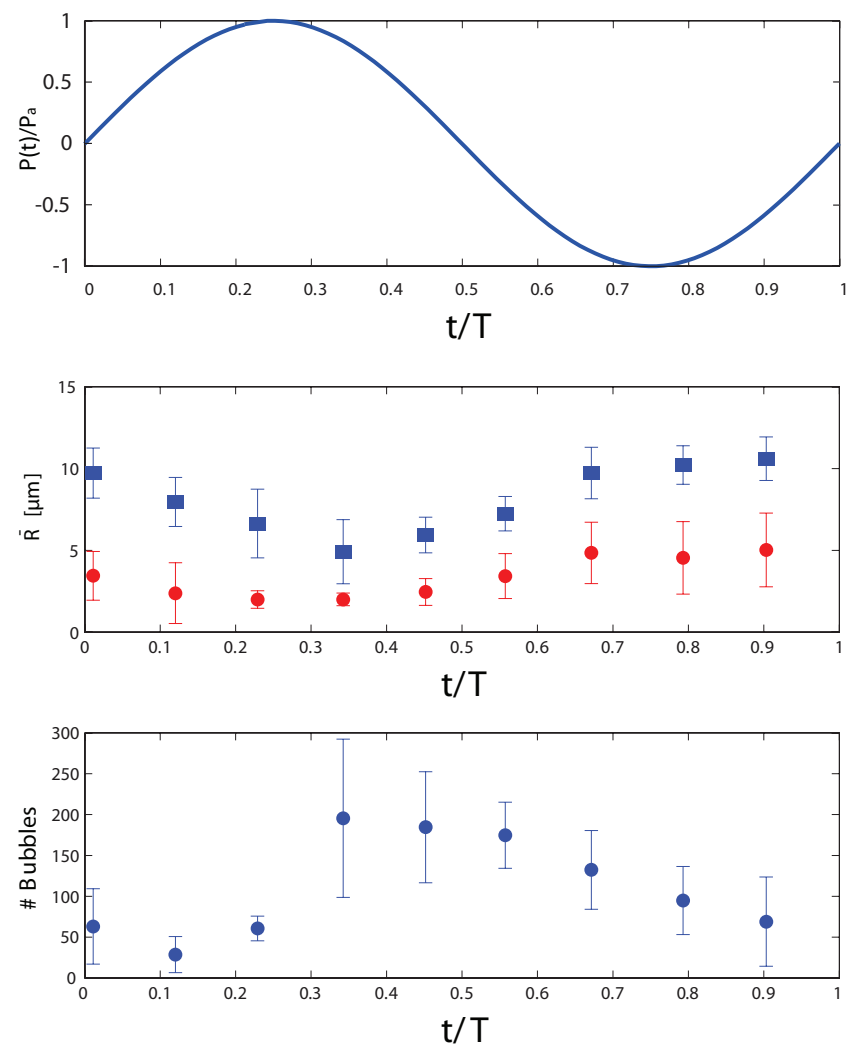

Figure 3.12: Top: Normalized pressure for the acoustic cycle. Center: Most probable radius (circles) and average radius (squares); Bottom: Average number of bubbles at different phases at a power of $0.981 \mathrm{~W}$ for three pits.

\subsubsection{Experimental radical production rates}

It was shown earlier [29] that the duration of the experiment does not seem to affect the reaction rates up to 30 minutes of operation. The radical production rate defined in Equation 3.1 is shown as a function of the ultrasonic power in Fig. 3.14. It is important to note that there is no significant effect of the US power when no pits are present. It is clear that the presence of the pits is essential for for the generation of sonochemically active bubbles: the pits enhance cavitation by facilitating the appearance of a larger number of bubbles, at a location in the reaction cell that otherwise would have no detectable bubbles or none at all.

For the lowest power the total radical production rates are around $5 \mathrm{nM} \mathrm{s}^{-1}$, but 
Table 3.1: Bubble population characteristics in a cycle.

\begin{tabular}{cccc}
\hline Low power $(0.194 \mathrm{~W})$ & & & \\
& 1 pit & 2 pits & 3 pit \\
\hline Most probable radius $[\mu \mathrm{m}]$ & 17 & 3 & 2 \\
Average bubble radius $[\mu \mathrm{m}]$ & 16 & 10 & 12 \\
$N_{\text {bubble }}$ & 3 & 5 & 17 \\
\hline Medium power $(0.654 \mathrm{~W})$ & & & \\
& 1 pit & 2 pits & 3 pit \\
\hline Most probable radius $[\mu \mathrm{m}]$ & 2 & 3 & 3 \\
Average bubble radius $[\mu \mathrm{m}]$ & 9 & 12 & 8 \\
$N_{\text {bubble }}$ & 4 & 29 & 113 \\
\hline High power $(0.981 \mathrm{~W})$ & 1 pit & 2 pits & 3 pit \\
\hline Most probable radius $[\mu \mathrm{m}]$ & 2 & 3 & 5 \\
Average bubble radius $[\mu \mathrm{m}]$ & 8 & 9 & 10 \\
$N_{\text {bubble }}$ & 50 & 102 & 96 \\
\hline
\end{tabular}

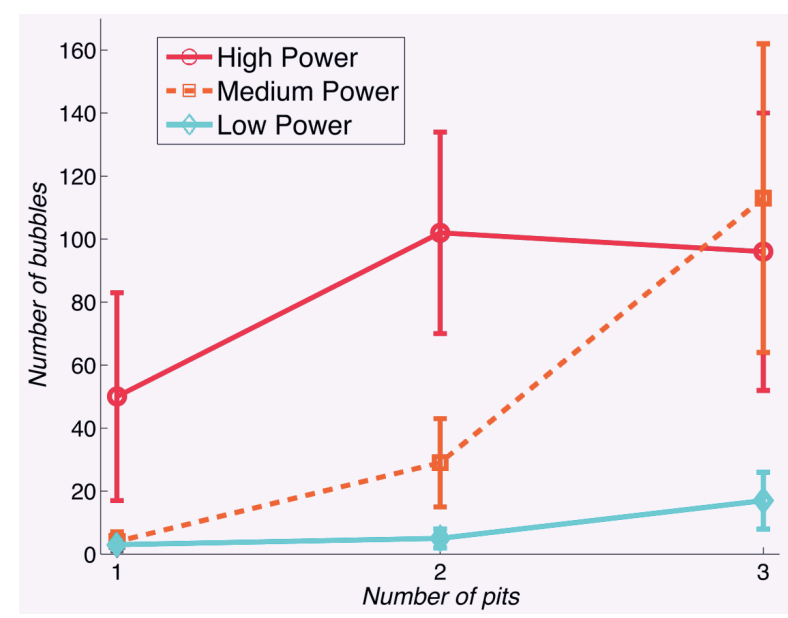

Figure 3.13: Average number of bubbles in the cavitation field for the different power settings: Low $(0.194 \mathrm{~W})$, Medium $(0.654 \mathrm{~W})$ and High $(0.981 \mathrm{~W})$.

as the power is increased, the reaction rate increases as well. The maximum value corresponds to the three-pits configuration at high power.

When the amount of radicals formed per second is divided by the number of pits we see an interesting trend depicted in Fig. 3.15. For one pit the rate of radical formation is higher than for two- and three-pits in all cases. Possible factors contributing to 


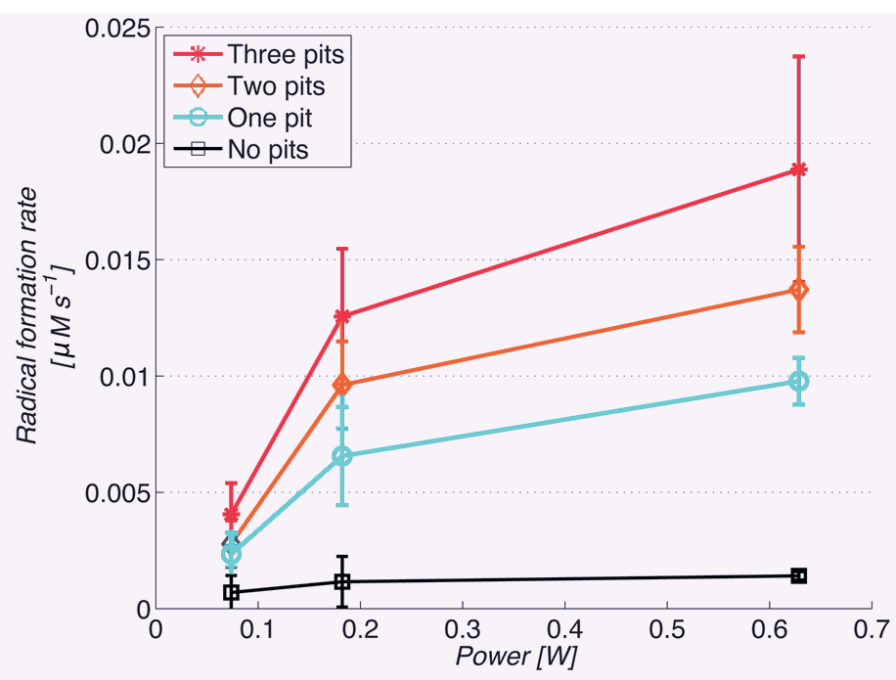

Figure 3.14: Radical production rates for the different power settings.

this result are the mutual shielding of the bubbles, a detuning of the cell and change in the sound power distribution due to the larger gas volume fraction, the availability of dissolved gas in the liquid and possibly others.

If we calculate the radical production per cycle and divide it by the average number of bubbles per cycle, we can get an estimate to be compared with single bubble radical production measured by Didenko and Suslick [6] who reported data for a single bubble of maximum radius of $28.9 \mu \mathrm{m}$ driven at $52 \mathrm{kHz}$. They measured an $\mathrm{OH} \cdot$ radical generation rate of $6.6 \times 10^{5}$ per cycle. Our data, which are shown in Table 3.2, are of the same order of magnitude despite the differences in the experimental parameters. We expected lower rates due to the smaller volume of our bubbles (itself a consequence of the higher frequency), to mutual shielding and bubble deformation. A possible explanation for our results is radical production by bubbles smaller than $0.5 \mu \mathrm{m}$, which our experimental technique cannot resolve.

\subsubsection{Discussion of the calculated efficiencies}

In principle, a measure suitable for the quantification of the sonochemical conversion could be defined as:

$$
X_{U S}=\frac{\Delta H\left(\Delta N_{\text {rad }} / \Delta t\right)}{P_{U S}},
$$




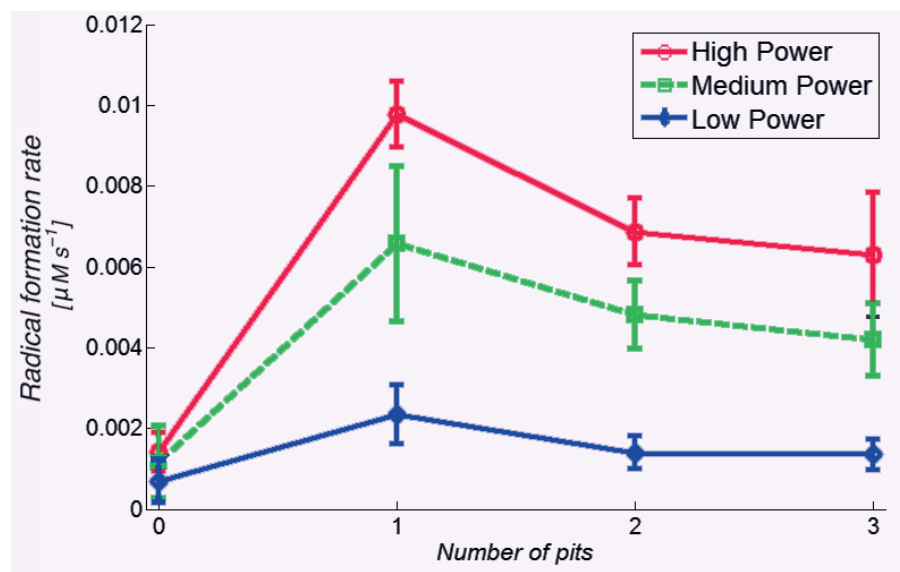

Figure 3.15: Radical production rates for the different power settings (Low $0.074 \mathrm{~W}$, Medium $0.182 \mathrm{~W}$ and High $0.629 \mathrm{~W}$ ) divided by the number of pits.

Table 3.2: Radical production per detected bubble per cycle

\begin{tabular}{cccc}
\hline Low power & & & \\
$(0.074-0.194 \mathrm{~W})$ & 1 pit & 2 pits & 3 pit \\
& $7.06 \times 10^{5}$ & $5.03 \times 10^{5}$ & $2.15 \times 10^{5}$ \\
\hline Medium power & & & \\
$(0.182-0.654 \mathrm{~W})$ & 1 pit & 2 pits & 3 pit \\
& $1.48 \times 10^{6}$ & $2.99 \times 10^{5}$ & $1.00 \times 10^{5}$ \\
\hline High power & & & \\
$(0.629-0.981 \mathrm{~W})$ & 1 pit & 2 pits & 3 pit \\
& $1.76 \times 10^{5}$ & $1.21 \times 10^{5}$ & $1.78 \times 10^{5}$ \\
\hline
\end{tabular}

where $P_{U S}$ is the power absorbed by the transducer and $\Delta H$ is the energy required for the formation of $\mathrm{OH}$ radicals $\left(5.1 \mathrm{eV}=8.2 \times 10^{-19} \mathrm{~J}\right)$, which is equal to the enthalpy of formation associated with the following chemical reaction:

$$
\mathrm{H}_{2} \mathrm{O} \stackrel{\Delta H=-5.1 \mathrm{eV}}{\rightarrow} \mathrm{OH}+\mathrm{H}^{\cdot}
$$

The problem with this definition is that only a small fraction of the power provided to the piezo is responsible for the sonochemical effect [56]. As a mater of fact, by comparing the power absorbed with and without pits and, therefore, in the presence or absence of intense bubble activity, we have found very small differences. This leads us to conclude that the majority of the power absorbed by the system is lost in the electrical-to-mechanical conversion. Nevertheless we will use the definition (3.2) as 


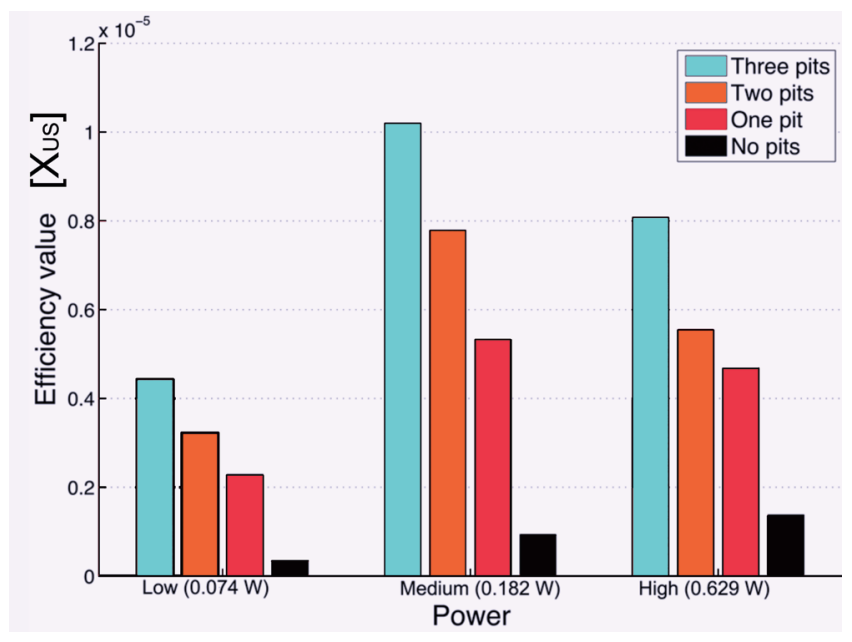

Figure 3.16: Nominal experimental efficiency values $\left(X_{U S}\right)$ for different number of pits and different US powers calculated from Eq. (3.2). The presence of pits for each power makes a clear difference with an increase in efficiency as the number of pits is increased. In the case of no pits, an increase in power produces a slow increase in efficiency. From low to medium power the trends increase for any number of pits. For the high power case, there appears to be a decrease in efficiency for any number of pits. It is not clear whether this result corresponds to true differences in the acoustic and sonochemical processes or to a decreased efficiency of conversion from electrical to mechanical power.

a convenient dimensionless measure of the sonochemical conversion efficiency. The electric power absorbed by the transducer $P_{U S}$ was determined from the measured voltage and current, and their phase difference.

The efficiency values calculated with Eq. (3.2) are shown in Fig. 3.16. As presented before [29], the most efficient setting appears to correspond to medium power. However, for the reasons mentioned before, the efficiencies shown in the figure may not reflect purely acoustic features of the system. Nevertheless we present this data following the prevailing custom in the sonochemical literature $[27,56]$.

\subsubsection{Obtaining numerical effective bubble size distributions and effec- tive driving pressures}

As explained in Section 3.2.5, one can estimate the production of radicals in a single bubble on the basis of existing models. In order to apply these models to the complex 
bubble fields studied in the present experiments, it is necessary to know the distribution of bubble radii and the effective pressure amplitude to which each bubble is subjected.

For this purpose we relied on measured probability distributions of bubble sizes at each phase of the sound field. These PDF were obtained by collecting 10 snapshots of the bubble field for each phase of the pressure. Each snapshot can be considered as statistically equivalent and independent from the others as the separation between successive ones was $2 \times 10^{5}$ cycles which is much longer than the expected lifetime of the individual bubbles. The idea of the simulation was to determine a pressure amplitude and bubble equilibrium radius distribution that matched the measured radii distribution at each phase.

We started from the measured distribution at a particular phase chosen so as to have a large number of bubbles, which generally coincided with a small average radius and small standard deviation. For each one of these bubble radii we integrated the extended Rayleigh-Plesset equation assuming a vanishing initial radial velocity and using different pressure amplitudes [39]. We found that the nominal pressure amplitudes applied in the experiment produced bubble size distributions quite different from the measured ones, as shown in Fig. 3.17.

In order to match the measured distributions (see Figs. 3.9, 3.10 and 3.11) it was necessary to adjust the pressure amplitudes used in the simulations, which resulted in much lower values than the nominal experimental ones. The adjustment was done by minimizing the cumulative squared difference between experimental and calculated PDF for each phase (see Fig. 3.18).

The pressure levels deduced in this way were in the range between $110 \mathrm{kPa}$ and $170 \mathrm{kPa}$. Since the minimum radius detectable in the experiment was about $0.5 \mu \mathrm{m}$, a larger number of small bubbles appeared in simulations than in the experiment. The fit between experimental and calculated PDF was best for high applied power and more pits, i.e. for high numbers of nucleated bubbles (see Fig. 3.19 and 3.20).

At low applied power the number of bubbles detected in the experiments was too small to provide good statistics, especially in the one-pit configuration. Moreover, at low power, the standard deviations of both the experimental and the calculated PDF normalized by the average radii were higher during the whole oscillation cycle, even if the average radii themselves were larger. This means that the data were more scattered than in the high power case, both in absolute and relative terms.

In the one-pit case, if we increased the applied power, the effective pressure derived from simulations monotonically increased (see Fig. 3.21) together with the number of bubbles measured in the experiments, while their average radii and normalized standard deviations decreased. In the two- and three-pits cases, a similar 

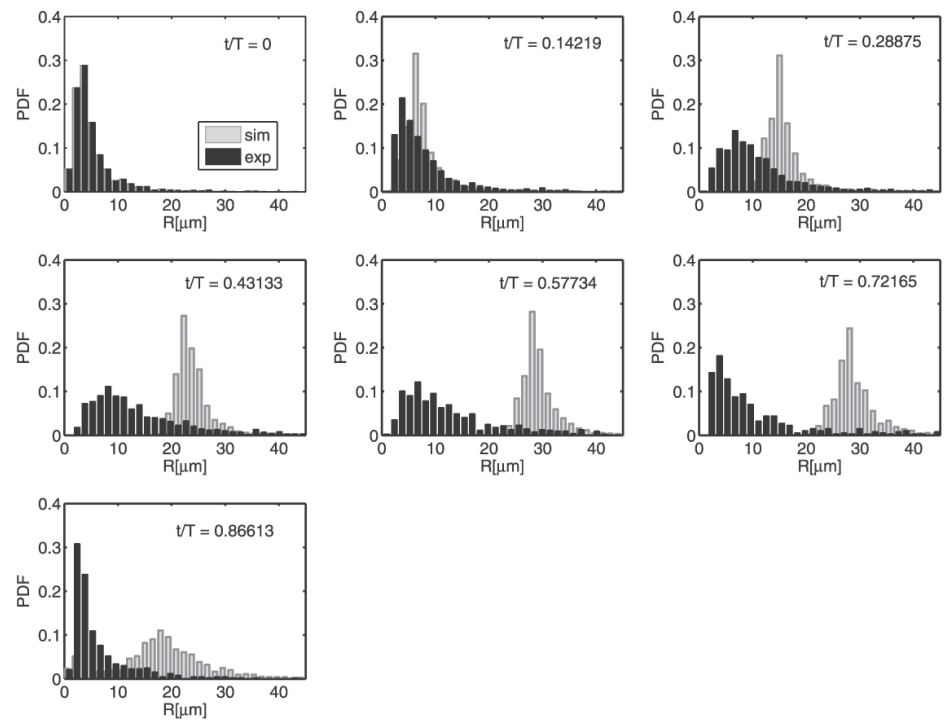

Figure 3.17: Bubble size distribution from experiment (black) and simulation (grey), for high power $(0.981 \mathrm{~W})$ and the three-pit configuration. It appears that the pressure amplitude measured with the hydrophone overestimated the effective value felt by the bubble population. Simulations carried out using these values were not able to describe the evolution in time of the radii distribution, as the average radius was overestimated during the whole cycle, the expansion phase was much longer than in experiments and the distributions were almost symmetric, instead of right-skewed.

behavior was observed, with the effective pressure rising with the applied power and the average radius decreasing.

This trend, however, changed in correspondence of the pressure levels at which the observed bubble distribution switched from separate clouds surrounding each pit to the complex bubble field structure observed in Fig. 3.3 and Fig. 3.4. Beyond this threshold, the measured bubble size distribution changed significantly and the pressure necessary to fit the data abruptly decreased. This supports our view that the mutual shielding of the bubbles leads to a reduction in the effective pressure seen by the bubbles.

\subsubsection{Calculation of the radical production}

On the basis of the bubble sizes and pressure amplitudes determined in the way described in Subsection 8.2, we calculated then the number of $\mathrm{OH}$. radicals produced 


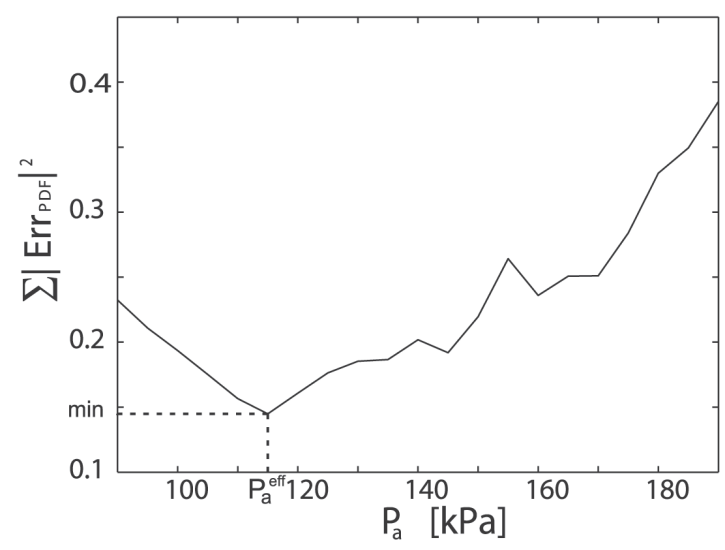

Figure 3.18: Cumulative squared error (over all bubble sizes and acoustic phases) between the PDF of the radii from experiments and from the simulations, as a function of the pressure values used in the simulations, for three-pit configuration, at high power $(0.981 \mathrm{~W})$. The effective driving pressure felt by the bubble population was $115 \mathrm{kPa}$ and was extracted by minimizing the cumulative squared error.

by singles bubble with the equilibrium radius corresponding to the experimental distribution.

The bubble population at the instant at which the average radius over the 10 frames was minimum was taken as the initial and equilibrium distribution for the simulations. This generally coincided with the instant when the highest number of bubbles was found.

For this calculation, one hundred equi-spaced bubble equilibrium radii were chosen for each $10 \mu \mathrm{m}$ size bin. For the pressure conditions given by the fitting procedure, the number of radicals diffusing inside the liquid over the first cycle was calculated for each value of the radius. The results were then averaged over all the radii belonging to the same bin.

As a general behavior, the calculated chemical output was higher at higher effective pressure but, in the two- and three-pits configurations, the radical production exhibited a significant dependence on the bubble size distribution, which was different before and after the transition from individual bubble clouds to complex patterns. Thus, a higher radical production was observed in the latter cases even for the same calculated effective pressure (see Fig. 3.22).

The transition to the complex bubbles patterns affects the acoustic field and the liquid flow in the cell, with possible consequences for the sonochemical activity as 

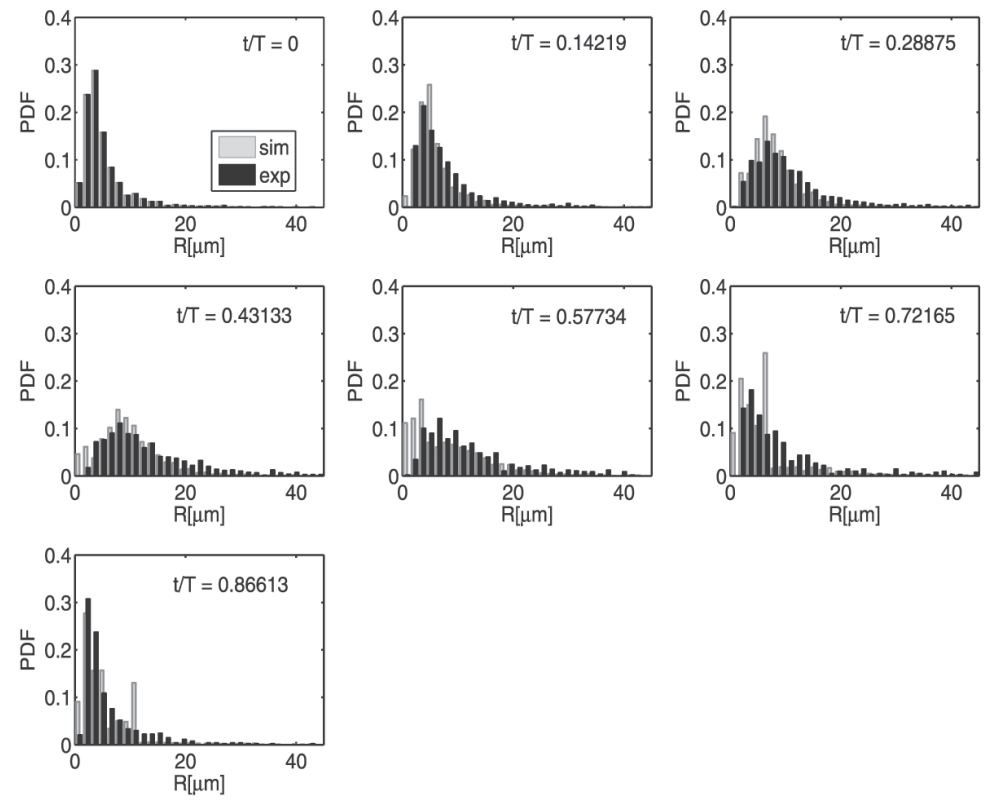

Figure 3.19: Bubble size distribution from experiment (black) and simulation (grey), for high power $(0.981 \mathrm{~W})$ and the three-pit configuration. The effective pressure amplitude extracted of $115 \mathrm{kPa}$ provided a good description of the dynamics of the system. The agreement between experiments and simulations was best for more pits and high applied power, i.e. for high number of nucleated bubbles. As expected, a higher number of small sized bubbles appeared in simulations than in experiments, as the minimum bubble radius that the experimental apparatus could detect was 0.5 $\mu \mathrm{m}$.

found in earlier studies [57].

In all the cases examined the whole $\mathrm{OH}$ - radical production was found to come from bubbles smaller than resonance size, i.e. smaller than $15 \mu \mathrm{m}$, and mainly from those with a radius around $5 \mu \mathrm{m}$ (see Fig. 3.23, 3.24, 3.25). In particular, higher effective pressure amplitudes resulted in smaller sizes of the bubbles giving the peak chemical production. This effect is dominant with respect to differences in bubble volume and the surface area of the bubble across which radicals diffuse.

The experimental rates span over one decade, while the theoretical rates shown in Figure 3.22 span six decades. The reason for this is that the experimental precision is much lower than that of the numerical calculations, hence the strength of combining these two approaches. 

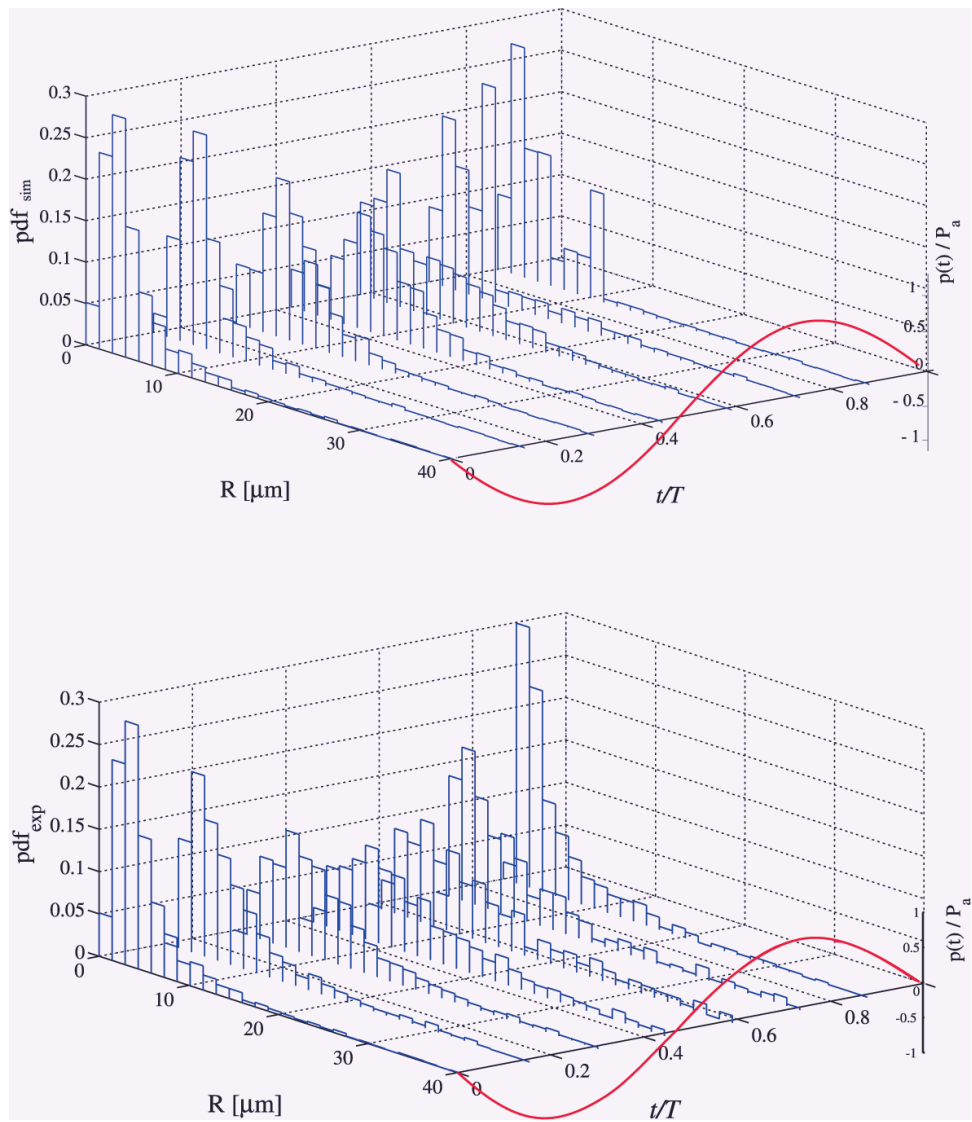

Figure 3.20: Bubble size distribution histograms at high power $(0.981 \mathrm{~W})$ for three pits. From experiments (lower figure), and from simulations (upper figure) with the deduced effective pressure $(115 \mathrm{kPa})$, which gives the best fit to the observed bubble distribution. 


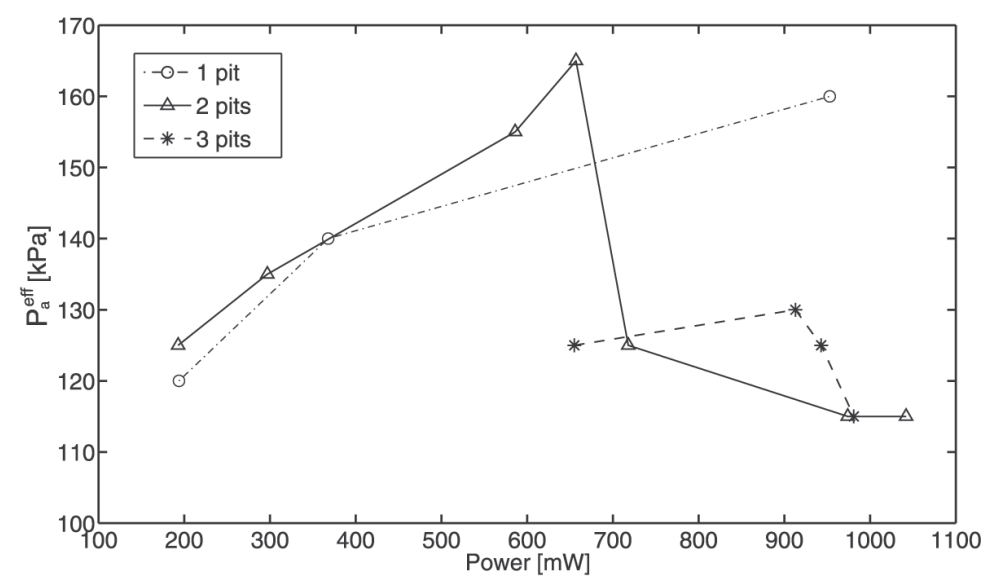

Figure 3.21: Effective driving pressure felt by the bubble population as a function of the applied power. These values were derived from our fitting procedure between experimental and calculated PDF of the radii (see Fig. 3.18). For different number of pits, bubbles started to appear for different applied powers in experiments, although corresponding to the same deduced effective pressure (around $125 \mathrm{kPa}$ ). In the onepit configuration, the effective pressure monotonically grew with the electric power. In the two- and three-pit cases the effective pressure initially had a similar behavior, but it abruptly decreased when the bubbles started to migrate towards the center point of the pit array above $0.657 \mathrm{~W}$ and $0.914 \mathrm{~W}$ respectively.

It must be noted that our spherical bubble model overestimates the reaction rates measured in the experiments. This is perhaps not surprising given that the many factors that induce non-spherical collapse and therefore limit the compression of the gas are absent from the simulation. The simulations should be regarded as establishing an upper limit to the reaction rates. In principle, it may be thought that non-spherical bubble models could reduce the gap between simulations and experiments, although the complexity of the experimental situation will probably lie beyond any practical model in the foreseeable future. 

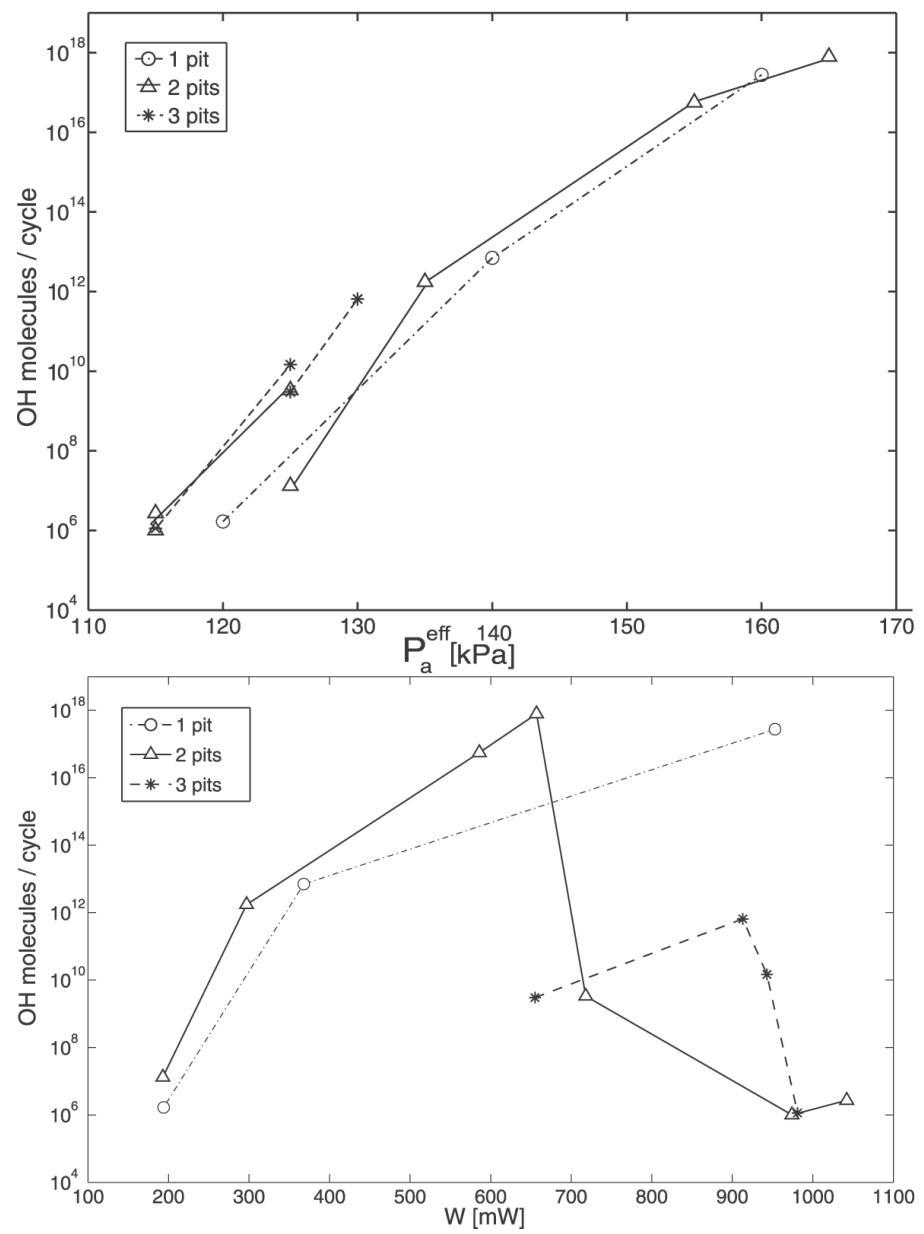

Figure 3.22: Number of $\mathrm{OH}$ - radicals diffusing into the liquid, in each acoustic cycle, as a function of pressure (upper figure) and applied power (lower figure), both calculated from simulations. The calculated chemical production increased with the effective pressure, but also depended on the PDF of the radii. In the two- and three-pit cases, when the bubbles migrated towards the center point of the pit array (at 0.657 $\mathrm{W}$ and $0.914 \mathrm{~W}$ ), more radicals were produced than when they remain near the pits , although the effective pressure was the same $(125 \mathrm{kPa})$. In the upper figure this corresponds to the first branch of the two- and three-pit curves (below $125 \mathrm{kPa}$ ). The right branches correspond to the initial rise of the power. 

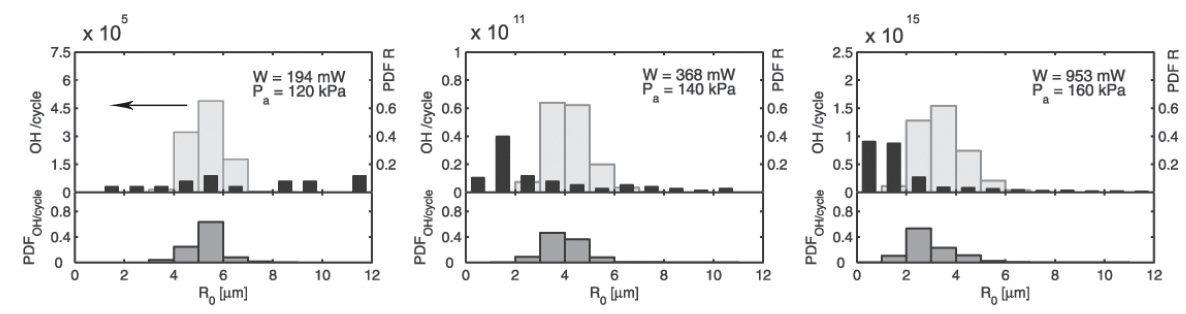

Figure 3.23: One-pit case. Upper figures, light grey: calculated number of $\mathrm{OH}$. radicals diffusing into the liquid at each acoustic cycle per bubble, as a function of the initial radius $\mathrm{R}_{0}$ for a single bubble (see Section 7.3 for the details). Upper figures, black: PDF of the initial experimental distributions of the radii in the one-pit case, for different applied powers. Lower figures, dark grey: PDF of the $\mathrm{OH} \cdot$ radicals diffusing into the liquid in each acoustic cycle, as a function of the initial radius. These values were obtained by normalizing the number of the $\mathrm{OH} /$ cycle produced by every bin of initial radii over the total number of $\mathrm{OH} /$ cycle from the specific distribution. These lower figures can be seen as a combination of the two upper graphs. Simulations showed that the most radical production came from bubbles smaller than resonant size $(15 \mu \mathrm{m})$. In particular, higher effective pressure values corresponded to smaller sizes of the most active bubbles. 

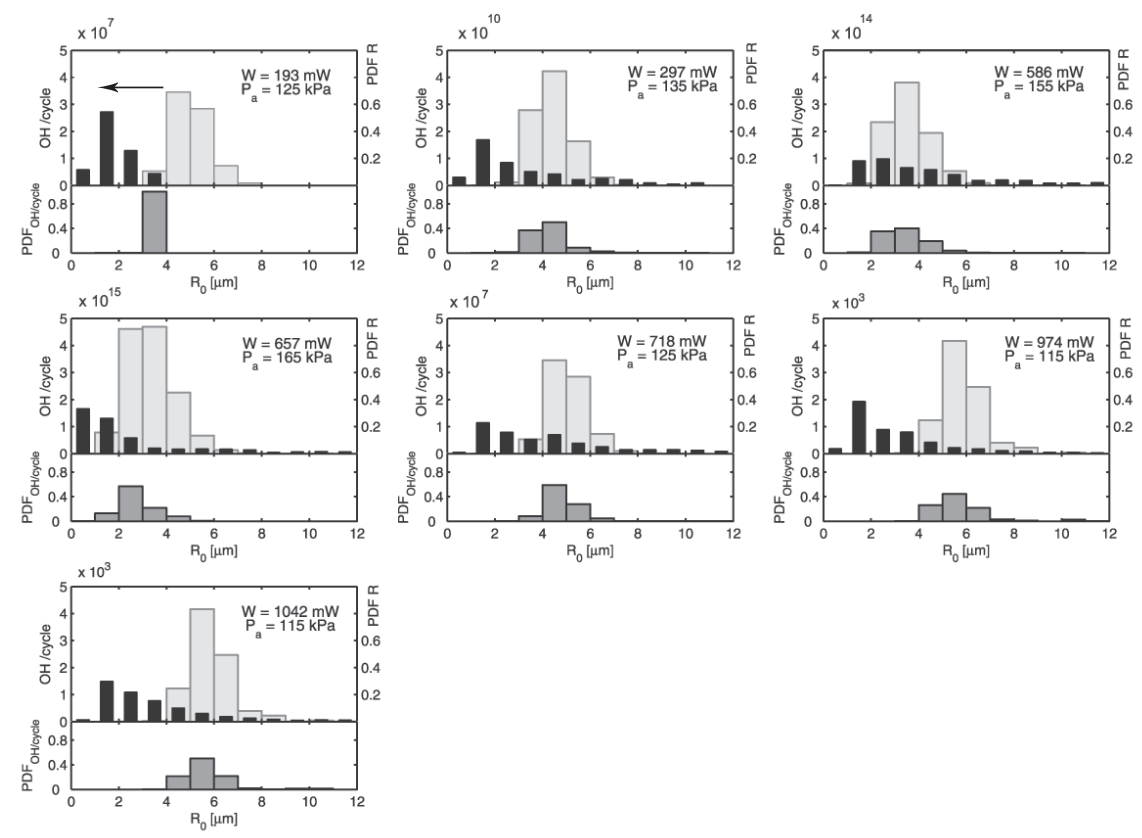

Figure 3.24: Same as Fig. 3.23 for the two-pit case. 

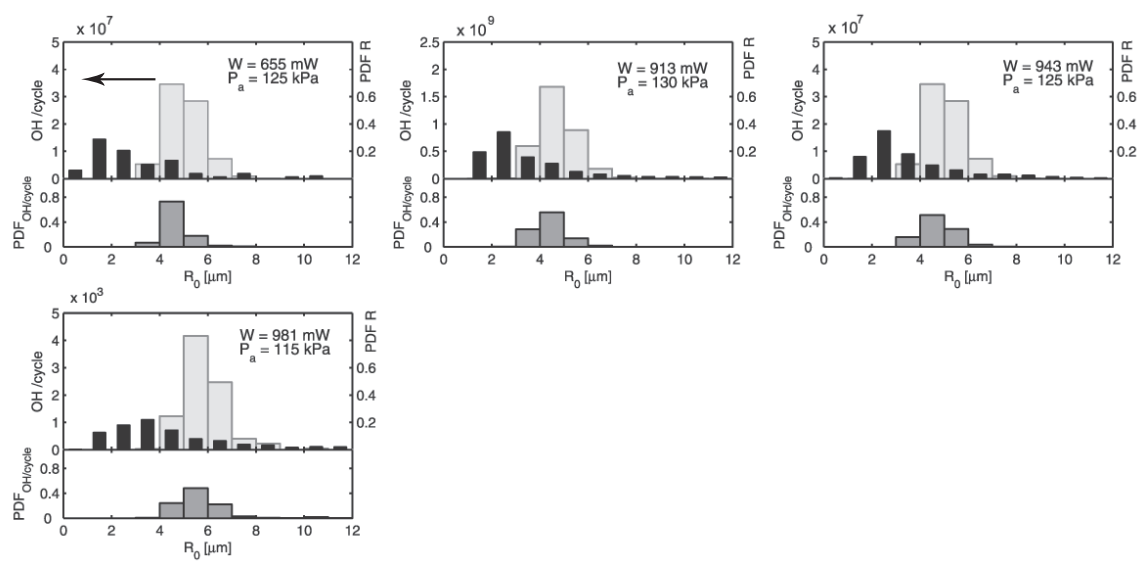

Figure 3.25: Same as Fig. 3.23 for the three-pit case.

\subsection{Summary and Conclusions}

We achieved a more controllable and more efficient sonoreactor design by microfabricating small pits on a silicon chip immersed in a small sonoreactor of the ultrasonic bath type. Under the action of an ultrasonic field, the air trapped in the pits gives rise to a stream of small bubbles which are responsible for the intense sonochemical activity. A rectified diffusion process is responsible for replenishing the air and maintaining the activity of the pit.

We have shown that this modification of the solid surface yields considerably higher conversion rates. We have measured the bubble size distribution at different power settings and characterized its evolution during the acoustic cycle.

Simulations were carried out in order to give an estimate of the radical production in the different power settings. By fitting the experimental and the calculated PDF of the radii and minimizing the cumulative squared differences between the two, an estimate of the effective forcing pressure felt by the bubbles was found. In the two- and three-pit cases the effective pressure dropped when bubbles started to migrate towards the center point of the pit array. Simulations also allowed us to give a description of the temporal evolution of the smaller bubbles below $3 \mu$ m radius, compatible with the experimental PDF. This turned out to be particularly relevant, as calculations showed that the most $\mathrm{OH}$. radical production came from the smaller bubbles around $5 \mu \mathrm{m}$ radius. As a general trend, the calculated chemical rate increased monotonically with the effective pressure.

Even though the numerical model considers spherical bubbles without interac- 
tion, an important strength lies in the possibility of taking the actual experimental bubble size distribution (with its equivalent radius corresponding to non-spherical bubbles) and giving information on the smallest bubbles that are below the experimental observability. This computation can be considered as a useful complement to the experiment since it permits one to estimate the local pressure value to which the bubbles are exposed and which cannot be directly measured with the available pressure sensors.

It may be possible to extend the present surface modification to larger scale sonoreactors by supplying similar nucleation sites on larger surfaces and in a much greater number. As a guide towards the development and optimization of these types of reactors, it will be useful to investigate in greater detail several aspects such as pit density, dimensions, mutual interaction and many others. 


\section{References}

[1] M. Ashokkumar, T. J. Mason, Sonochemistry, John Wiley \& Sons, Inc., 2000.

[2] A. Gedanken, Sonochemistry and its application to nanochemistry, Curr. Sci. 85 (12) (2003) 1730-1734.

[3] N. Mahamuni, Y. Adewuyi, Advanced oxidation processes (AOPs) involving ultrasound for waste water treatment: A review with emphasis on cost estimation, Ultrason. Sonochem. 17 (6) (2010) 990-1003.

[4] M. Ashokkumar, D. Sunartio, S. Kentish, R. Mawson, L. Simons, K. Vilkhu, C. Versteeg, Modification of food ingredients by ultrasound to improve functionality: A preliminary study on a model system, Innov. Food Sci. Emerg, Technol. 9 (2) (2008) 155-160.

[5] D. Lohse, Sonoluminescence-Cavitation hots up, Nature 434 (2005) 33-34.

[6] Y. T. Didenko, K. S. Suslick, The energy efficiency of formation of photons, radicals and ions during single-bubble cavitation, Nature 418 (6896) (2002) 394-397.

[7] U. Parlitz, R. Mettin, S. Luther, I. Akhatov, M. Voss, W. Lauterborn, Spatiotemporal dynamics of acoustic cavitation bubble clouds, Phil. Trans. R. Soc. Lond. A 357 (1751) (1999) 313.

[8] J. Appel, P. Koch, R. Mettin, D. Krefting, W. Lauterborn, Stereoscopic highspeed recording of bubble filaments, Ultrason. Sonochem. 11 (1) (2004) 39-42.

[9] M. P. Brenner, S. Hilgenfeldt, D. Lohse, Single-bubble sonoluminescence, Rev. Mod. Phys. 74 (2002) 425-484.

[10] K. S. Suslick, D. J. Flannigan, Inside a collapsing bubble: Sonoluminescence and the conditions during cavitation, Annu. Rev. Phys. Chem. 59 (2008) 659683.

[11] K. Suslick, N. Eddingsaas, D. Flannigan, S. Hopkins, H. Xu, Extreme Conditions during Multibubble Cavitation: Sonoluminescence as a Spectroscopic Probe, Ultrason. Sonochem. 18 (2011) 842 - 846.

[12] R. Pflieger, H. Brau, S. Nikitenko, Sonoluminescence from $\mathrm{OH}(\mathrm{C} 2 \Sigma+)$ and $\mathrm{OH}$ $(\mathrm{A} 2 \Sigma+)$ Radicals in Water: Evidence for Plasma Formation during Multibubble Cavitation, Chem. Eur. J. 16 (39) (2010) 11801-11803. 
[13] F. Burdin, N. Tsochatzidis, P. Guiraud, A. Wilhelm, H. Delmas, Characterisation of the acoustic cavitation cloud by two laser techniques, Ultrason. Sonochem. 6 (1-2) (1999) 43-51.

[14] S. Luther, R. Mettin, P. Koch, W. Lauterborn, Observation of acoustic cavitation bubbles at 2250 frames per second, Ultrason. Sonochem. 8 (3) (2001) 159-162.

[15] N. Tsochatzidis, P. Guiraud, A. Wilhelm, H. Delmas, Determination of velocity, size and concentration of ultrasonic cavitation bubbles by the phase-Doppler technique, Chem. Eng. Sci. 56 (5) (2001) 1831-1840.

[16] S. Labouret, J. Frohly, Bubble size distribution estimation via void rate dissipation in gas saturated liquid. Application to ultrasonic cavitation bubble fields, Eur. Phys. J. AP 19 (01) (2002) 39-54.

[17] W. Chen, T. Matula, L. Crum, The disappearance of ultrasound contrast bubbles: observations of bubble dissolution and cavitation nucleation, Ultrasound Med. Biol 28 (6) (2002) 793-803.

[18] D. Sunartio, M. Ashokkumar, F. Grieser, Study of the coalescence of acoustic bubbles as a function of frequency, power, and water-soluble additives, J. Am. Chem. Soc. 129 (18) (2007) 6031-6036.

[19] A. Brotchie, F. Grieser, M. Ashokkumar, Effect of power and frequency on bubble-size distributions in acoustic cavitation, Phys. Rev. Lett. 102 (8) (2009) 84302 .

[20] C. E. Brennen, Cavitation and Bubble Dynamics, Oxford University Press, Oxford, 1995.

[21] T. G. Leighton, The acoustic bubble, Academic Press, London, 1994.

[22] M. Ashokkumar, The Characterization of Acoustic Cavitation Bubbles-An Overview, Ultrason. Sonochem. 2010.

[23] R. Tronson, M. Ashokkumar, F. Grieser, Comparison of the effects of watersoluble solutes on multibubble sonoluminescence generated in aqueous solutions by 20 -and 515-kHz pulsed ultrasound, J. Phys. Chem. B 106 (42) (2002) $11064-11068$.

[24] K. Okitsu, T. Suzuki, N. Takenaka, H. Bandow, R. Nishimura, Y. Maeda, Acoustic multibubble cavitation in water: A new aspect of the effect of a rare 
gas atmosphere on bubble temperature and its relevance to sonochemistry, J. Phys. Chem. B 110 (41) (2006) 20081-20084.

[25] T. Tuziuti, K. Yasui, Y. Iida, Spatial study on a multibubble system for sonochemistry by laser-light scattering, Ultrason. Sonochem. 12 (1-2) (2005) 73-77.

[26] S. Hatanaka, K. Yasui, T. Kozuka, T. Tuziuti, H. Mitome, Influence of bubble clustering on multibubble sonoluminescence, Ultrasonics 40 (1-8) (2002) 655660 .

[27] M. W. A. Kuijpers, M. F. Kemmere, J. T. F. Keurentjes, Calorimetric study of the energy efficiency for ultrasound-induced radical formation, Ultrasonics 40 (1-8) (2002) 675-678.

[28] Z. Zeravcic, D. Lohse, W. van Saarloos, Collective oscillations in bubble clouds, J. Fluid Mech. 680 (2011) 114-149.

[29] D. Fernández Rivas, A. Prosperetti, A. G. Zijlstra, D. Lohse, H. J. G. E. Gardeniers, Efficient sonochemistry through microbubbles generated with micromachined surfaces, Angew. Chem. Int. Ed. 49 (50) (2010) 9699-9701.

[30] D. Fernández Rivas, Taming acoustic cavitation, Ph.D. thesis, University of Twente (2012).

[31] N. Bremond, M. Arora, C. D. Ohl, D. Lohse, Controlled multi-bubble surface cavitation, Phys. Rev. Lett. 96 (2006) 224501.

[32] N. Bremond, M. Arora, S. M. Dammer, D. Lohse, Interaction of cavitation bubbles on a wall, Phys. Fluids 18 (12) (2006).

[33] P. Marmottant, J. P. Raven, H. Gardeniers, J. G. Bomer, S. Hilgenfeldt, Microfluidics with ultrasound-driven bubbles, J. Fluid Mech. 568 (2006) 109-118.

[34] B. M. Borkent, S. Gekle, A. Prosperetti, D. Lohse, Nucleation threshold and deactivation mechanisms of nanoscopic cavitation nuclei, Phys. Fluids 21 (10) (2009).

[35] A. van der Bos, A. Zijlstra, E. Gelderblom, M. Versluis, iLIF: illumination by Laser-Induced Fluorescence for single flash imaging on a nanoseconds timescale, Exp. Fluids 51 (2011) 1283-1289.

[36] F. Meyer, Topographic distance and watershed lines, Signal Process. 38 (1994) $113-125$. 
[37] T. J. Mason, J. P. Lorimer, D. M. Bates, Y. Zhao, Dosimetry in sonochemistry - the use of aqueous terephthalate ion as a fluorescence monitor, Ultrason. Sonochem. 1 (2) (1994) S91-S95.

[38] Y. Iida, K. Yasui, T. Tuziuti, M. Sivakumar, Sonochemistry and its dosimetry, Microchem. J. 80 (2) (2005) 159-164.

[39] A. Prosperetti and A. Lezzi, Bubble dynamics in a compressible liquid. Part 1. First-order theory, J. Fluid Mech. 168 (1986) 457-478.

[40] R. Toegel, S. Hilgenfeldt, D. Lohse, Suppressing dissociation in sonoluminescing bubbles: The effect of excluded volume, Phys. Rev. Lett. 88 (2002) 034301.

[41] R. Toegel, D. Lohse, Phase diagrams for sonoluminescing bubbles: A comparison between experiment and theory, J. Chem. Phys. 118 (2003) 1863.

[42] L. Stricker, A. Prosperetti, D. Lohse, Validation of an approximate model for the thermal behavior in acoustically driven bubbles, J. Acoust. Soc. Am. 130 (5) (2011) 3243-3251.

[43] T. G. Leighton, Bubble population phenomena in acoustic cavitation, Ultrason. Sonochem. 2 (2) (1995) S123-S136.

[44] E. A. Neppiras, Acoustic cavitation, Phys. Rep. 61 (3) (1980) 159-251.

[45] A. Zijlstra, Acoustic surface cavitation, Ph.D. thesis, University of Twente (2011).

[46] H. Gelderblom, A. Zijlstra, L. van Wijngaarden, A. Prosperetti, Oscillations of a gas pocket on a liquid-covered solid surface, Phys. Fluids 24 (2012) 122101.

[47] A. Moussatov, C. Granger, B. Dubus, Cone-like bubble formation in ultrasonic cavitation field, Ultrason. Sonochem. 10 (4-5) (2003) 191-195.

[48] A. Moussatov, R. Mettin, C. Granger, T. Tervo, B. Dubus, W. Lauterborn, Evolution of acoustic cavitation structures near larger emitting surface, in: Proc. 5th World Congress on Ultrasonics, Paris, France, 2003, pp. 955-958.

[49] R. Mettin, S. Luther, C. Ohl, W. Lauterborn, Acoustic cavitation structures and simulations by a particle model, Ultrason. Sonochem. 6 (1-2) (1999) 25-29.

[50] R. Pecha, B. Gompf, Microimplosions: Cavitation collapse and shock wave emission on a nanosecond time scale, Phys. Rev. Lett. 84 (2000) 1328-1330. 
[51] M. P. Brenner, S. Hilgenfeldt, D. Lohse, Single bubble sonoluminescence, Rev. Mod. Phys. 74 (2002) 425-484.

[52] N. Bremond, M. Arora, S. M. Dammer, D. Lohse, Interaction of cavitation bubbles on a wall, Phys. Fluids 18 (12) (2006) 121505.

[53] S. Uzel, M. A. Chappell, S. J. Payne, Modeling the cycles of growth and detachment of bubbles in carbonated beverages, J. Phys. Chem. B 110 (14) (2006) 7579-7586.

[54] J. B. Fowlkes, L. A. Crum, Cavitation threshold measurements for microsecond length pulses of ultrasound, J. Acoust. Soc. Am. 83 (6) (1988) 2190-2201.

[55] L. Crum, Nucleation and stabilization of microbubbles in liquids, Appl. Sci. Res. 38 (1) (1982) 101-115.

[56] J. Berlan, T. J. Mason, Sonochemistry: from research laboratories to industrial plants, Ultrasonics 30 (4) (1992) 203 - 212.

[57] S. Hatanaka, H. Mitome, K. Yasui, S. Hayashi, Multibubble sonoluminescence enhancement by fluid flow, Ultrasonics 44 (2006) e435-e438. 


\section{4}

\section{Radical production inside an acoustically driven microbubble *}

The chemical production of radicals inside acoustically driven bubbles is determined by the local temperature inside the bubbles and by their composition at collapse. By means of a previously validated ODE model [1], based on boundary layer assumption for mass and heat transport, we study the influence of different parameters on the radical production. We perform different simulations by changing the driving frequency and pressure, the temperature of the surrounding liquid and the composition of the gas inside the bubbles. In agreement with the experimental conditions of new generation sonochemical reactors, where the bubbles undergo transient cavitation oscillations [2], we mainly concentrate on the initial chemical transient and we suggest optimal working ranges for technological applications. The importance of the chemical composition at collapse is reflected in the model, including the role of entrapped water vapor. We in particular study the exothermal reactions taking place in $\mathrm{H}_{2}$ and $\mathrm{O}_{2}$ mixtures. At the exact stoichiometric mixture 2:1 the highest internal bubble temperatures are achieved.

*Submitted to Ultrason. Sonochem. as: [Laura Stricker and Detlef Lohse, Radical production inside an acoustically driven microbubble]. 


\subsection{Introduction}

An acoustically driven microbubble can produce extreme conditions of temperature and pressure, thus giving rise to emission of light ("sonoluminescence", SL ) [3-7] and chemical conversions, involving the gas inside the bubble and the surrounding liquid ("sonochemistry") [8-13]. Chemical reactions also play the main role in stabilizing single sonoluminescent air bubbles [14, 15]

The chemical output, as well as the light emission, is highly influenced by the experimental parameters such as the driving pressure and frequency, the initial saturation concentration, the liquid temperature and pressure and the kind and amount of dissolved gas. For technological applications, a deep understanding of these dependencies is crucial in order to optimize the radical production.

A number of studies of the frequency effect have been published, some with conflicting results. Experiments on multi-bubble systems (MBSL) reported an increase in $\mathrm{OH}$ radical production with increasing frequency $[16,17]$ up to an optimal value around $300-400 \mathrm{kHz}[18,19]$. On the other side, experiments on single sonoluminescent bubbles (SBSL) revealed that the radical production decreased as the frequency increased [20]. These two results may seem contradictory but can be explained considering that, at low frequency, the bubbles grow bigger and their collapses are more violent, often leading to transient cavitation. Therefore each bubble undergoes only few oscillations before fragmentation at these low frequencies and it is mainly filled with water vapor $[21,22]$. Conversely, at high frequency stable cavitation is generally the main form of cavitation: bubbles exist for a long period of time and they contain mainly the gas originally dissolved in water [21, 23]. Moreover, the different sizes of the nucleated bubbles have to be considered: the bubbles are smaller at higher frequency and therefore possibly more chemically active [2]. Bubble-bubble interaction and its induced flow could also play a role on the chemical activity, which is however not yet understood.

Other relevant parameters to the radical production are the conditions of the liquid. It has been calculated that there is an optimal bulk temperature for radical production both with air [24] and argon bubbles [25]. This trend is related to the competing effects of the entrapped water vapor, that decreases the bubble temperature at collapse [26] but also increases the amount of reactants. This trend was retrieved in experiments both with water $[16,18,27]$ and organic solvents, such as methanol/water mixtures [28].

The dependence of the radical production on the ambient pressure has been studied both experimentally [29, 30], and theoretically [31], showing that decreasing the ambient pressure has the same effects than increasing the driving amplitude, as a higher radical production follows $[18,20]$. Some authors reported an asymptotic be- 
havior of the oxidants production with increasing driving pressures and ascribed it to the entrapped vapor [32].

Many authors examined the effect of the kind and composition of the dissolved gas, both experimentally [18, 19, 33-35] and numerically [25]. Studies of different kinds of noble gases [33, 34] have established a correlation between the light emission, and therefore the temperature at collapse, and the thermal conductivity of the gas; as the latter increases, more heat can escape from the bubble at collapse, thus reducing the peak temperature. Investigations over different mixtures of a noble and a diatomic gas reported higher reaction rates for large percentages of the noble gas, both from experiments $[18,19]$ and numerics [25]. This behavior was ascribed to the larger adiabatic exponent $\gamma$ of the mixture: as $\gamma$ rises, so does the temperature at collapse.

However, direct quantitative comparisons between the mentioned theoretical studies on single bubble sonochemistry and experiments have always been problematic. Few experimental data are available on the radical production of a single air bubble in water $[36,37]$. Such experiments are difficult to perform in controlled conditions, because of the low amount of radicals produced per cycle and the long operating time required to detect any chemical output (some hours). In order to compare calculations with experiments, theoretical studies mainly concentrate on steady cavitation [38]. Some numerical papers include data on the early transient phase [39, 40] but, to our knowledge, no systematic parametric study has been published on that yet.

Nevertheless, we believe that such a study can be crucial for technological applications, as in new generation efficient sonochemical multibubble reactors the bubbles are nucleated from oscillating menisci and present a lifetime of few acoustic cycles [2]. In our previous work [2], we considered each bubble as an independent sonochemical reactor and we used the bubbles themselves to measure the effective driving pressure. In the present work we concentrate on the transient state and we carry on an extensive scan of the parametric range. We also examine the temperatures inside the bubbles and we try to correlate it to the radical production for different gases. Yasui et al. $[32,40]$ calculated that, for air bubbles, there is an optimal range of bubble temperature at collapse (between 4000-6500 K) where oxidants production is maximum. If the temperature raises too much (above $7000 \mathrm{~K}$ ) the sonochemical rate decreases due to oxidizing nitrogen reactions. Our results confirm this observation. Moreover, we found that under that threshold, the relevant element to chemical conversion is the temperature, as its increase always corresponds to an increase in the chemical output. Over that threshold, the dependence is not so clear anymore and other elements, such as the reactants concentration, become more relevant.

In our study, we use the simplified ODE model based on boundary layer approx- 
imation, previously developed in Refs. [26, 41, 42] and validated in Ref. [1], which was also found to be in good agreement with results by Storey and Szeri [43]. This model includes the Rayleigh-Plesset equation for the radial dynamics of the bubble, van der Waals law for the inner pressure, the energy equation for the temperature, heat and mass fluxes, water evaporation/condensation, changes of transport parameters according to changes of the mixture composition and a complete set of non-equilibrium chemical reactions for gas and water.

Despite the high level of simplification, this model manages to reproduce various features of chemical reactions, such as the explosive nature of stoichiometric mixtures of oxygen and hydrogen. Therefore we are confident that this approach could lead to realistic estimates of the radical production rate of single spherical collapsing bubbles and considerable increased efficiencies.

\subsection{Model}

We use an ODE model, based on the boundary layer approximation, previously developed in [22, 41, 42] and validated in [1]. We give here a summarizing description and we refer the reader to those papers for additional details.

We assume that the bubble contains a perfect gas with a spatially uniform temperature and pressure, i.e. low Mach numbers. We also assume spherical symmetry and shape stability, thus restricting our study to a range of parameters where these assumptions are reasonable [44, 45]

We use the Rayleigh-Plesset equation to describe the radial dynamics of the bubble:

$$
\begin{aligned}
& \left(1-\frac{\dot{R}}{c}\right) R \ddot{R}+\frac{3}{2}\left(1-\frac{\dot{R}}{3 c}\right) \dot{R}^{2} \\
& \quad=\frac{1}{\rho}\left(1+\frac{\dot{R}}{c}\right)\left(p-p_{\infty}-P(t)\right)+\frac{R \dot{p}}{\rho c}-\frac{4 v \dot{R}}{R}-\frac{2 \sigma}{\rho R}
\end{aligned}
$$

The dot denotes time derivatives, $R$ is the bubble radius, $c$ is the speed of sound, $\rho$ and $v$ are the density and the kinematic viscosity of the liquid respectively, $\sigma$ is the surface tension, $p_{\infty}$ is the static pressure and $P(t)=P_{a} \cos \omega t$, with $P_{a}$ the acoustic driving amplitude and $f=\omega / 2 \pi$ the frequency.

The gas pressure inside the bubble $p$ is calculated from a modified van der Waals equation of state taking into account the gas inertia [42]:

$$
p=\frac{N_{t o t} k_{B} T}{V-N_{t o t} B}-\frac{1}{2} \rho_{B} R \ddot{R},
$$


in which $N_{t o t}$ is the total number of gas molecules, $\rho_{B}$ is the gas density, $k_{B}$ the Boltzmann constant, and $B$ the molecular co-volume.

The temperature $T$ is determined by means of an energy balance over the bubble volume:

$$
\dot{E}=\dot{Q}-p \dot{V}+\sum_{i}\left(h_{w, i}+e_{\text {form }, i}\right) \dot{N}_{i}^{d}
$$

where $E$ is the total energy of the bubble, $V$ is the bubble volume, $\dot{N}_{i}^{d}$ the number of particles of component $i$ diffusing inside the bubble per unite time, $h_{w, i}$ the molecular enthalpy of component $i$ at the cold bubble wall and $e_{f o r m, i}$ its enthalpy of formation. The net heat absorbed by the bubble per unit time $\dot{Q}$ is provided by

$$
\begin{aligned}
\dot{Q} & =4 \pi R^{2} \lambda_{m i x} \frac{T_{0}-T}{l_{t h}}, \\
l_{t h} & =\min \left(\sqrt{\frac{R \chi}{|\dot{R}|}}, \frac{R}{\pi}\right)
\end{aligned}
$$

with $\lambda_{\text {mix }}$ the thermal conductivity of the gas mixture, $l_{t h}$ an estimate of the thickness of the thermal boundary layer inside the bubble [41, 42] and $T_{0}$ the temperature of the liquid. Similarly the diffusive flux of component $i$ is expressed by

$$
\begin{aligned}
\dot{N}_{i}^{d} & =4 \pi R^{2} D_{i} \frac{n_{i, 0}-n_{i}}{l_{i, d}}, \\
l_{i, d} & =\min \left(\sqrt{\frac{R D_{i}}{|\dot{R}|}}, \frac{R}{\pi}\right)
\end{aligned}
$$

with $D_{i}$ the mass diffusivity of specie $i$ in the mixture of all the other species, $l_{i, d}$ its mass diffusive boundary layer thickness, $n_{i}$ the time dependent concentration of particles of specie $i$ per unit volume and $n_{i, 0}$ the respective equilibrium concentration at bubble wall. For the moderately soluble species, i.e. $\mathrm{H}_{2}, \mathrm{O}_{2}, \mathrm{~N}_{2}$ and $\operatorname{argon}, n_{i, 0}=n_{i, \infty}$ derived from the saturation of the liquid at infinite. For the highly soluble species, that is all the others except water, we take $n_{i, 0}=0$. For water, the equilibrium concentration $n_{i, 0}$ is derived from the saturation pressure of the vapor at temperature $T_{0}$ : $n_{H_{2} O, 0}=P_{v}\left(T_{0}\right) / k_{B} T_{0}$. This treatment incorporates both evaporation/condensation at bubble wall and vapor/water diffusion from the wall to the core of the bubble. Details of calculation of transport parameters $\lambda_{\text {mix }}, \chi$ and $D_{i}$ can be found in Ref. [42].

As chemical reactions take place, the total energy of the bubble $E$ is not purely thermal but it is given by:

$$
E=\sum_{i}\left(e_{t h, i}+e_{f o r m, i}\right) N_{i}
$$


with $e_{t h, i}$ the thermal energy per molecule, calculated following Ref.[42]. Therefore Eq.(4.3) becomes

$$
\dot{T} \sum_{i} \frac{\partial e_{t h, i}}{\partial T} N_{i}=\dot{Q}-p \dot{V}+\sum_{i}\left(h_{w, i}+e_{f o r m, i}\right) \dot{N}_{i}^{d}+V \sum_{j} r_{j} \Delta E_{j}-\sum_{i} e_{t h, i} \dot{N}_{i}^{c},
$$

where $\Delta E_{j}$ is the energy variation associated to reaction $j$, taken as positive for exothermal reactions, negative for endothermal ones. $N_{i}^{c}$ is the change in the number of particles of specie $i$ per unit time, due to chemical reactions, expressed as

$$
\dot{N}_{i}^{c}=V \sum_{j} \alpha_{i, j} r_{j}
$$

in which $\alpha_{i, j}$ is the stoichiometric change of specie $i$ in reaction $j$ and $r_{j}$ is the net rate of reaction $j$, given by

$$
r_{j}=r_{f, j}-r_{b, j}
$$

Here $r_{f, j}$ and $r_{b, j}$ are the forward and the backward rate respectively, calculated by means of Arrhenius law, following the reaction scheme of Ref. [42]. For eg., for a reaction of the kind $\mathbf{A}+\mathbf{B} \leftrightarrow \mathbf{C}+\mathbf{D}$

$$
\begin{aligned}
& r_{f, j}=k_{f, j} n_{A} n_{B} T^{c_{f, j}} \exp \left(-\frac{E_{f, j}}{k_{B} T}\right), \\
& r_{b, j}=k_{b, j} n_{C} n_{D} T^{c_{b, j}} \exp \left(-\frac{E_{b, j}}{k_{B} T}\right),
\end{aligned}
$$

where $k_{f, j}, k_{b, j}, c_{f, j}$ and $c_{b, j}$ are listed constants.

\subsection{Results}

The radical production is strongly affected by the driving parameters as well as by the composition of the gas mixture inside the bubble. Though the dynamic and the thermal equilibrium are generally reached quite quickly ( $\leq 30$ cycles), the chemical steady state can require hundreds or thousand cycles to be achieved. By definition, when the equilibrium is reached, the net flux of all chemical species from the bubble to the liquid becomes zero. Therefore the number of radicals diffusing inside the liquid strongly depends on the considered part of the transient. In the present work, we mainly focused on the early transient because in last generation multi-bubble sonochemical reactors the bubble life time was estimated around one acoustic cycle, due to splitting and recombination phenomena [2].Two different quantities were examined to estimate the radical production: the peak value and the diffusion rate, i.e. the 
number of radicals diffusing into the liquid per time unit. The peak value provides a good upper approximation for the radical outcome in one cycle, as most bubbles will disappear after implosion, thus releasing their content inside the liquid. Nevertheless, the diffusion rate is the most relevant parameter, when we wish to compare the efficiency of devices operating at different driving frequencies or to study the long term behavior.

\subsubsection{Effects of the type of gas}

\section{Temperature, composition at collapse and $\mathrm{O}, \mathrm{OH}$ and $\mathrm{H}$ production}

We studied bubbles initially composed of argon, hydrogen, oxygen, air and nitrogen. Depending on the operating frequency and the kind of radicals that one is interested in, it can be more convenient to use one or another gas. Among these gases, for $\mathrm{H}$ radicals, the most effective one, at any frequency is argon, for $\mathrm{OH}$ radicals it is $\mathrm{O}_{2}$ below $40 \mathrm{kHz}$ and argon above, for $\mathrm{O}$ radicals it is $\mathrm{O}_{2}$ under $15 \mathrm{kHz}$ and argon above, see Fig. 4.1, in which we plot the peak $\mathrm{OH}$ production for different gases together with their peak temperature. When the temperature at collapse is low (as it happens at high frequency), the radical production mainly depends on the temperature itself, when it is high (as it happens at low frequency), the main factor contributing to the radical production is the number of reactants at collapse and, in particular, water vapor. Above $10 \mathrm{kHz}$, the gas with the highest temperature is argon, because it is the only monatomic gas of the analyzed ones. Therefore the adiabatic exponent is 1.67 , as compared to 1.4 for diatomic gases. However, at very low frequency (under $10 \mathrm{kHz}$ ) argon bubbles do not result in the highest temperatures at collapse anymore, because also water vapor and exothermal reactions of $\mathrm{H}, \mathrm{O}$ and $\mathrm{OH}$ enhanced by the dissociation of $\mathrm{H}_{2}$ and $\mathrm{O}_{2}$ play a role. The peak temperature follows the order: $\mathrm{H}_{2}>$ air $>\mathrm{Ar} \geq \mathrm{O}_{2}>\mathrm{N}_{2} . \mathrm{N}_{2}, \mathrm{O}_{2}$ and air have the same adiabatic exponent but different peak temperatures at low frequency. It is therefore clear that the difference in the adiabatic exponent is not the only source of difference in the thermal behavior of the considered gases. Other relevant parameters are the thermal and mass diffusive coefficients. To understand these trends we have to consider the diffusive properties of the gases. From our calculations for binary mixtures we found that the mass diffusivity coefficients of water vapor inside the examined gases during the collapse were $D_{H 2}>D_{\text {air }} \geq D_{O 2} \geq D_{N 2}>D_{A r}$ and the thermal conductivities were $K_{H 2}>K_{O 2}>K_{\text {air }} \geq K_{N 2}>K_{A r}$. A higher value of vapor diffusivity corresponds to a lower quantity of water vapor entrapped inside the bubble at collapse (see Fig. 4.2) and therefore to a higher peak temperature [22]. The reasons are both the lower polytropic exponent of vapor respect to gas and the endothermic nature of wa- 

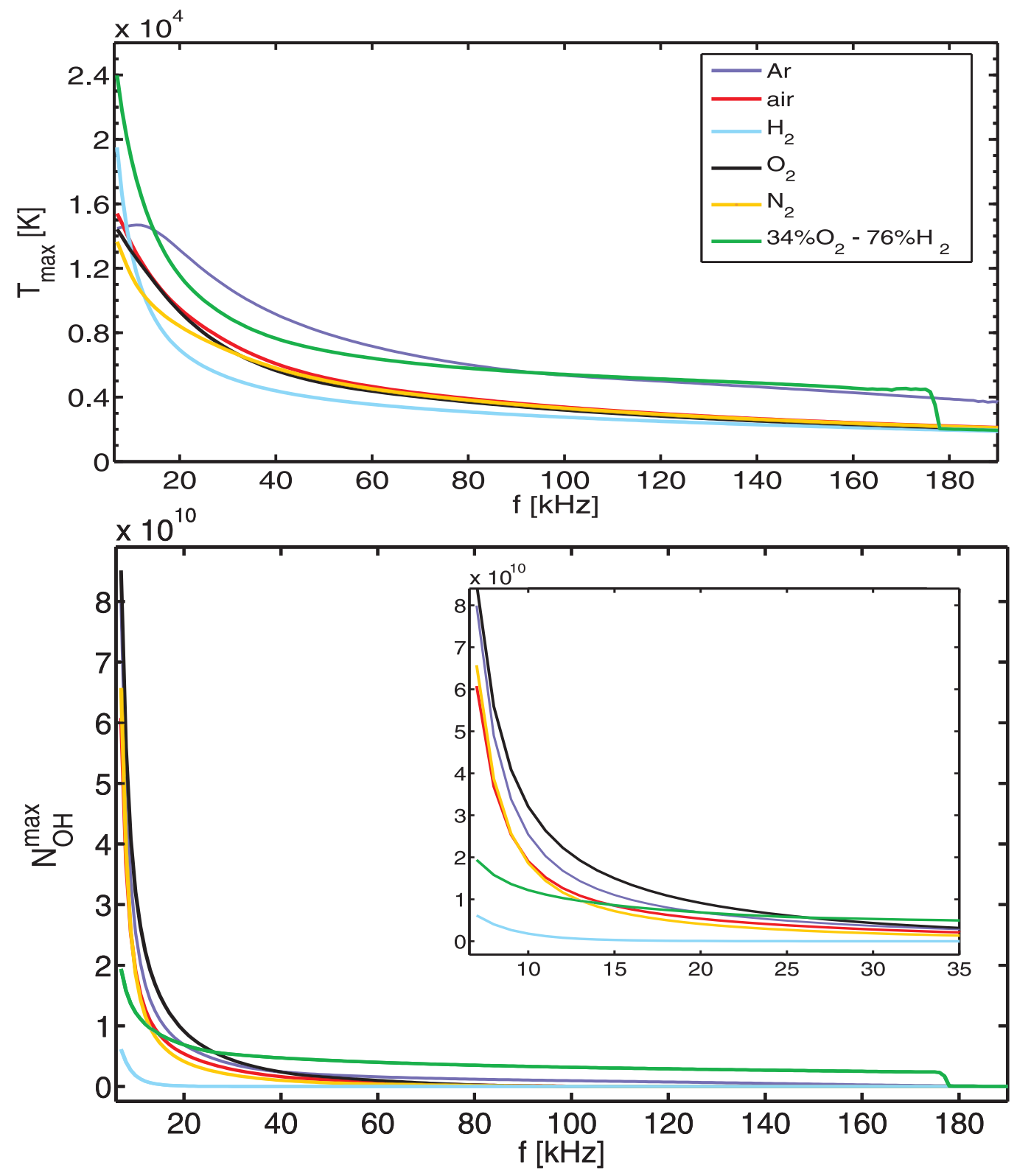

Figure 4.1: Maximum temperature (top) and number of $\mathrm{OH}$ molecules (bottom) during the first oscillating cycle of a bubble with the initial composition as indicated in the legend, as function of frequency $\left(\mathrm{R}_{0}=6 \mu \mathrm{m}, \mathrm{Pa}=1.4 \mathrm{~atm}\right)$. Above $40 \mathrm{kHz}$, the gas with the highest radical production is argon, under $40 \mathrm{kHz}$ it is $\mathrm{O}_{2}$. However, above $30 \mathrm{kHz}$, using a mixture of $\mathrm{H}_{2}-\mathrm{O}_{2}$ with stoichiometric water composition gives the highest chemical output, due to the activation of strongly exothermal hydrogen and oxygen chain reactions. 


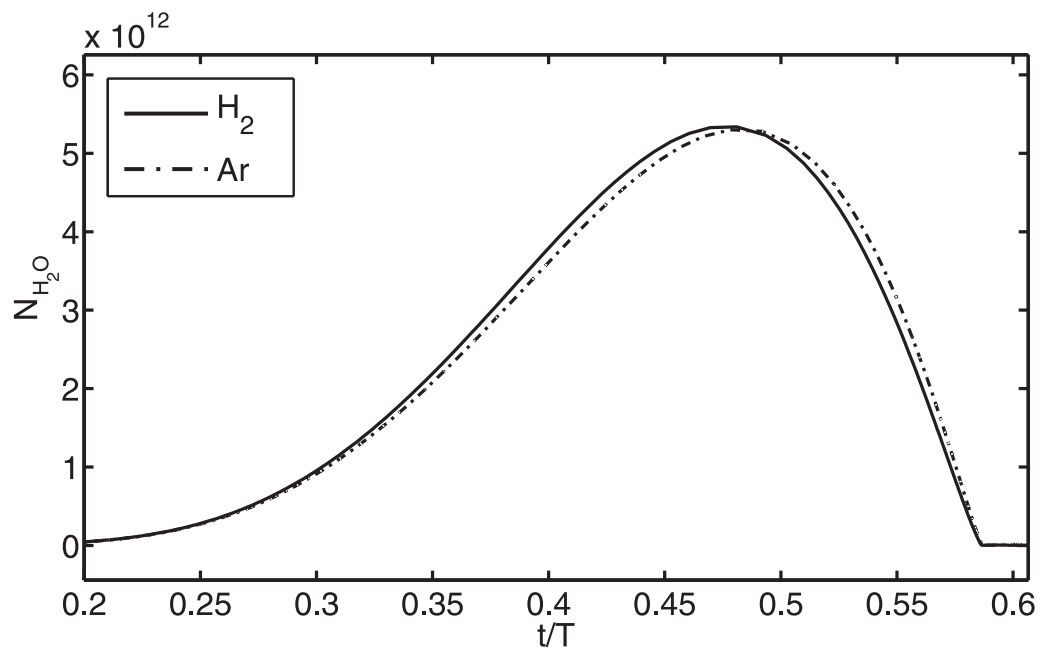

Figure 4.2: Number of water vapor molecules as function of time during the first oscillating cycle of a bubble initially composed of $\mathrm{H}_{2}$ (solid) and argon (dash-dot) with $R_{0}=6 \mu \mathrm{m}$, driven at $P_{a}=1.4 \mathrm{~atm}, f=7 \mathrm{kHz}$, with $T_{\infty}=20^{\circ} \mathrm{C}$. The diffusivity of water in $\mathrm{H}_{2}$ is higher than in argon, therefore less vapor is trapped in $\mathrm{H}_{2}$ bubbles at collapse. At low frequency, when the vapor has more time to diffuse inside the bubble during its expansion, this turns into a higher temperature peak for $\mathrm{H}_{2}$ bubbles (see Fig. 4.1).

ter dissociating reactions. Conversely, a higher thermal conductivity should provide a higher heat flux escaping from the bubble at collapse, therefore a lower temperature. In the case of $\mathrm{H}_{2}$ and argon bubbles, the temperature at collapse is lower for the latter and this is a proof of the higher effect of water mass diffusion respect to heat diffusion. The diffusivity of vapor inside $\mathrm{H}_{2}$ is almost 10 times larger than in argon, having an overwhelming effect respect to the different polytropic exponents and thermal conductivities. This becomes relevant at low frequencies, when the water vapor has more time to enter the bubble [22]. The dissociation reactions of $\mathrm{H}_{2}$ and $\mathrm{O}_{2}$, activated by the high temperatures reached at low frequency, also have a role, as they provide further reactants for exothermal recombination reactions of $\mathrm{H}, \mathrm{O}$ and $\mathrm{OH}$. Despite their high activation energy, the overall absorbed energy $\Delta E$ of $\mathrm{H}_{2}$ and $\mathrm{O}_{2}$ dissociation reactions is much lower than the overall released energy of $\mathrm{H}, \mathrm{O}$ and $\mathrm{OH}$ exothermal reactions. Therefore the global effect is an increase in temperature. This phenomenon is more relevant when the adopted gas is $\mathrm{H}_{2}$ or $\mathrm{O}_{2}$. Conversely to what was previously believed [34], the maximum temperature reached during the 
cycle is not always the main factor to determine the sonochemical production. This is true only at low temperatures. At high temperatures, the most relevant element is the amount of reactants available for dissociation, that is water vapor, $\mathrm{O}_{2}$ and $\mathrm{H}_{2}$. Therefore we can conclude that, at low frequencies (corresponding to high temperatures), the sonochemical production of $\mathrm{O}$ and $\mathrm{OH}$ radicals is mainly affected by the composition of the bubble at collapse, while, at high frequencies (corresponding to low temperatures), it mainly depends on the peak temperature itself.

Effects of argon percentage in air - argon mixtures: role of temperature and composition at collapse on $\mathbf{N}$ compound production

We examined a range of mixtures of air and argon from $0 \%$ to $100 \%$ Ar. At $200 \mathrm{kHz}$ the temperature raises monotonically because the polytropic exponent of the mixture increases with the argon percentage. The chemical activity starts only for argon percentages higher than $40 \%$, corresponding to a temperature at collapse of $2200 \mathrm{~K}$ (see Fig. 4.3). The production of $\mathrm{O}, \mathrm{OH}$ and $\mathrm{H}$ radicals follows the temperature trend, while the production of $\mathrm{N}, \mathrm{NH}, \mathrm{NH}_{2}, \mathrm{NH}_{3}, \mathrm{NO}$ and $\mathrm{NO}_{2}$ has a maximum at $90 \% \mathrm{Ar}$ and decreases to 0 for pure argon mixtures, as no nitrogen is then inside the system. This result is particularly relevant for technological applications, as most sonochemical reactors operate around this frequency. It is also remarkable that, in this range of temperatures (below $5000 \mathrm{~K}$ ), when the rate of nitrogen reactions is still very low, the key factor to nitrogen products is indeed the peak temperature and not the amount of reactants, as one could expect. At $20 \mathrm{kHz}$, this trend changes, because the temperatures are much higher. In agreement with Yasui et al. [40], we found that, after $7000 \mathrm{~K}$, nitrogen reactions are enhanced, becoming more relevant than water dissociation (see Fig. 4.4). Correspondingly, the amount of reactants for nitrogen reactions becomes more important and the optimal peaks of radical production are shifted towards lower amount of argon in the mixture. After $7000 \mathrm{~K}$, the amount of H,O and $\mathrm{OH}$ as well as the temperature, still grows with the argon percentage, though at a lower pace, because endothermal nitrogen oxidation reactions consume them. The maximum amount of $\mathrm{NO}$ and $\mathrm{NO}_{2}$ is found at $20 \% \mathrm{Ar}$, while the peak production of $\mathrm{N}$ and $\mathrm{NH}_{x}$ comes from $60 \%$ Ar mixtures. Considering all the total radical production, the optimal argon percentage range at $20 \mathrm{kHz}$ is between $40 \%$ and $60 \%$ Ar. For industrial application, one should remember that, lowering the frequency, the optimal concentration of argon decreases. 

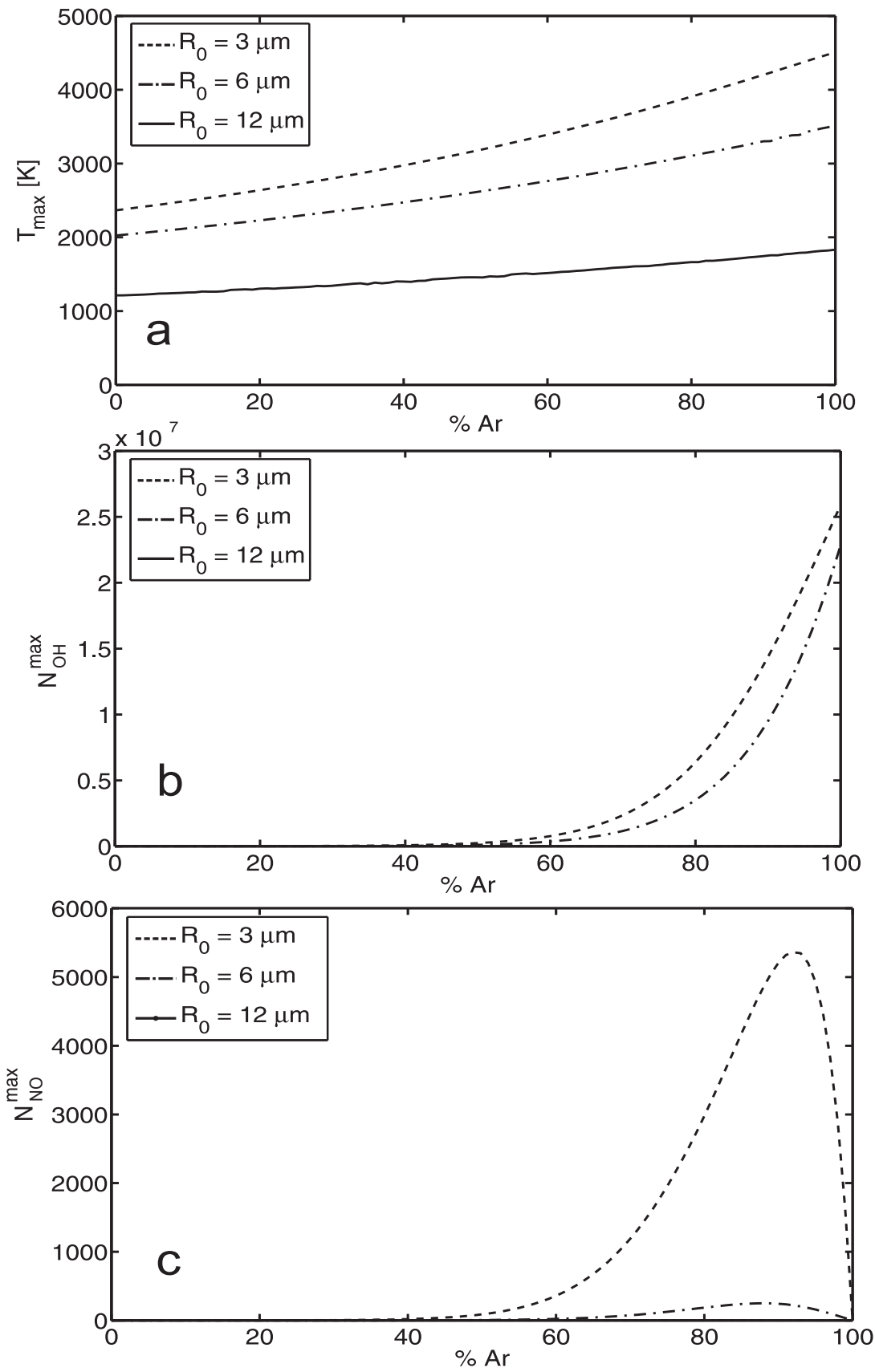

Figure 4.3: Maximum temperature (a), number of $\mathrm{OH}$ molecules (b) and number of NO molecules (c) during the first cycle of an air-argon bubble, as function of $\mathrm{Ar} \%$, at $200 \mathrm{kHz}\left(R_{0}=6 \mu \mathrm{m}, P_{a}=1.4 \mathrm{~atm}, T_{\infty}=20^{\circ} \mathrm{C}\right)$. At high frequency (corresponding to low temperatures), the radical production is mainly influenced by the peak temperature. Therefore, the optimal composition for radical production is around 90\% Ar. 

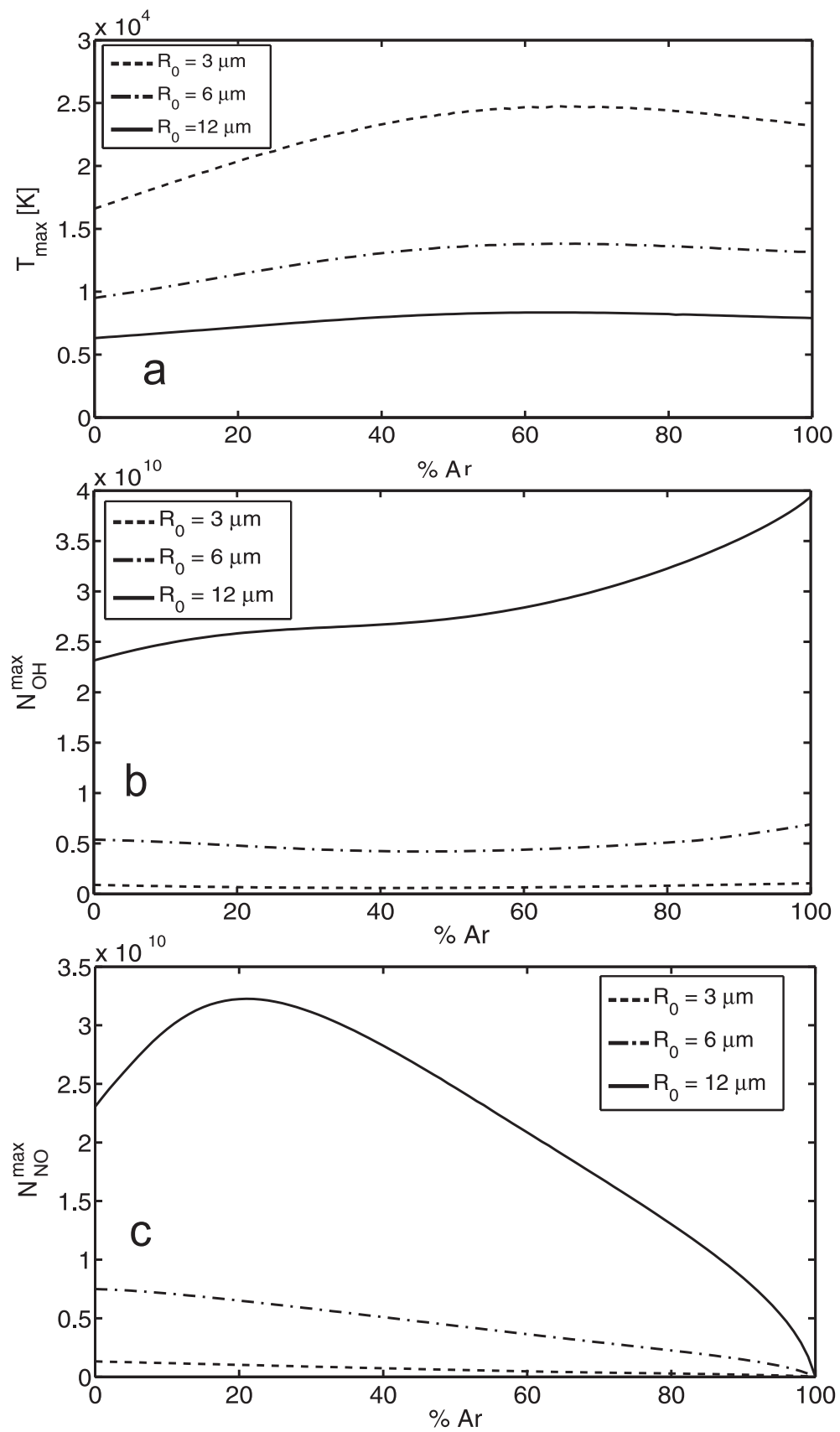

Figure 4.4: Maximum temperature (a), number of $\mathrm{OH}$ molecules (b) and number of NO molecules (c) during the first cycle of an air-argon bubble, as function of $\mathrm{Ar} \%$, at $20 \mathrm{kHz}\left(R_{0}=6 \mu \mathrm{m}, P_{a}=1.4 \mathrm{~atm}, T_{\infty}=20^{\circ} \mathrm{C}\right)$. At low frequency (corresponding to high temperatures), the radical production is mainly influenced by the composition of the bubble. Therefore, the optimal composition for radical production is around $20 \%$ Ar. 


\section{Effects of $\mathrm{O}_{2}$ percentage in $\mathrm{H}_{2}-\mathrm{O}_{2}$ mixtures: role of $\mathrm{H}_{2}$ and $\mathrm{O}_{2}$ dissociating reactions}

We examined the whole range of binary gas compositions, from pure $\mathrm{H}_{2}$ to pure $\mathrm{O}_{2}$. At $200 \mathrm{kHz}$, when the temperature remains lower than $2000 \mathrm{~K}$ for any composition, no reactions are initiated inside the bubble. Thus the only effect of adding $\mathrm{O}_{2}$ to the mixture is changing its diffusive properties, in particular lowering the thermal conductivity, as $\mathrm{O}_{2}$ has a lower thermal conductivity than $\mathrm{H}_{2}$. The water mass diffusivity into the gas changes as well, but it is a relevant parameter mainly at low frequencies (lower than $20 \mathrm{kHz}$ ), when the vapor has more time to diffuse inside the bubble during its expansion [26]. At $200 \mathrm{kHz}$, when the $\mathrm{O}_{2} \%$ increases, the reduction in thermal conductivity results into a slightly higher temperature at collapse, because the heat produced inside the bubble cannot escape easily (see Fig. 4.5).

At $20 \mathrm{kHz}$ a completely different behavior is found: the maximum temperature is reached for the a mixture of $2 / 3 \mathrm{H}_{2}$ and $1 / 3 \mathrm{O}_{2}$ in volume, that is the mixture with the stoichiometric composition of water vapor. In $6 \mu \mathrm{m}$ bubbles, such a mixture reaches a peak temperature of $11900 \mathrm{~K}$, which is higher than those of both pure $\mathrm{O}_{2}$ $(9322 \mathrm{~K})$ and pure $\mathrm{H}_{2}(6938 \mathrm{~K})$. In order to explain this phenomenon, we examine in the following a simplified example of chain reaction occurring in a $\mathrm{H}_{2}-\mathrm{O}_{2}$ mixture

\begin{tabular}{clccc}
\hline $11 \mathrm{j}$ & Reaction & $c_{j}$ & $E_{j} / k$ & $\Delta E_{j}$ \\
\hline 1 & $\mathrm{H}_{2}+\mathrm{M} \rightarrow \mathrm{M}+\mathrm{H}+\mathrm{H}$ & -0.8 & 52177 & -436 \\
2 & $\mathrm{O}_{2}+\mathrm{M} \rightarrow \mathrm{M}+\mathrm{O}+\mathrm{O}$ & -1.3 & 59893 & -498 \\
3 & $\mathrm{H}+\mathrm{O}_{2} \rightarrow \mathrm{O}+\mathrm{OH}$ & -0.7 & 8576 & -70 \\
4 & $\mathrm{O}+\mathrm{H}_{2} \rightarrow \mathrm{H}+\mathrm{OH}$ & 2.7 & 2200 & -8 \\
5 & $\mathrm{OH}+\mathrm{H}_{2} \rightarrow \mathrm{H}+\mathrm{H}_{2} \mathrm{O}$ & 1.5 & 1726 & 62 \\
6 & $\mathrm{H}+\mathrm{H}+\mathrm{M} \rightarrow \mathrm{H}_{2}+\mathrm{M}$ & -1 & 0 & 436 \\
7 & $\mathrm{O}+\mathrm{O}+\mathrm{M} \rightarrow \mathrm{O}_{2}+\mathrm{M}$ & -1 & 0 & 498 \\
8 & $\mathrm{H}+\mathrm{O}+\mathrm{M} \rightarrow \mathrm{OH}+\mathrm{M}$ & -1 & 0 & 428 \\
9 & $\mathrm{H}+\mathrm{OH}+\mathrm{M} \rightarrow \mathrm{H}_{2} \mathrm{O}+\mathrm{M}$ & -2 & 0 & 498
\end{tabular}

Table 4.1: Example of chain reactions in a $\mathrm{H}_{2}-\mathrm{O}_{2}$ mixture. $c_{j}$ are the power coefficients of the temperature in Eqs. (4.12), (4.13), $E_{j} / k$ are the activation temperatures, expressed in $\mathrm{K}$, and $\Delta E_{j}$ are the reaction energies in $\mathrm{kJ} / \mathrm{mol}$, positive for exothermal reactions and negative for endothermal ones. 

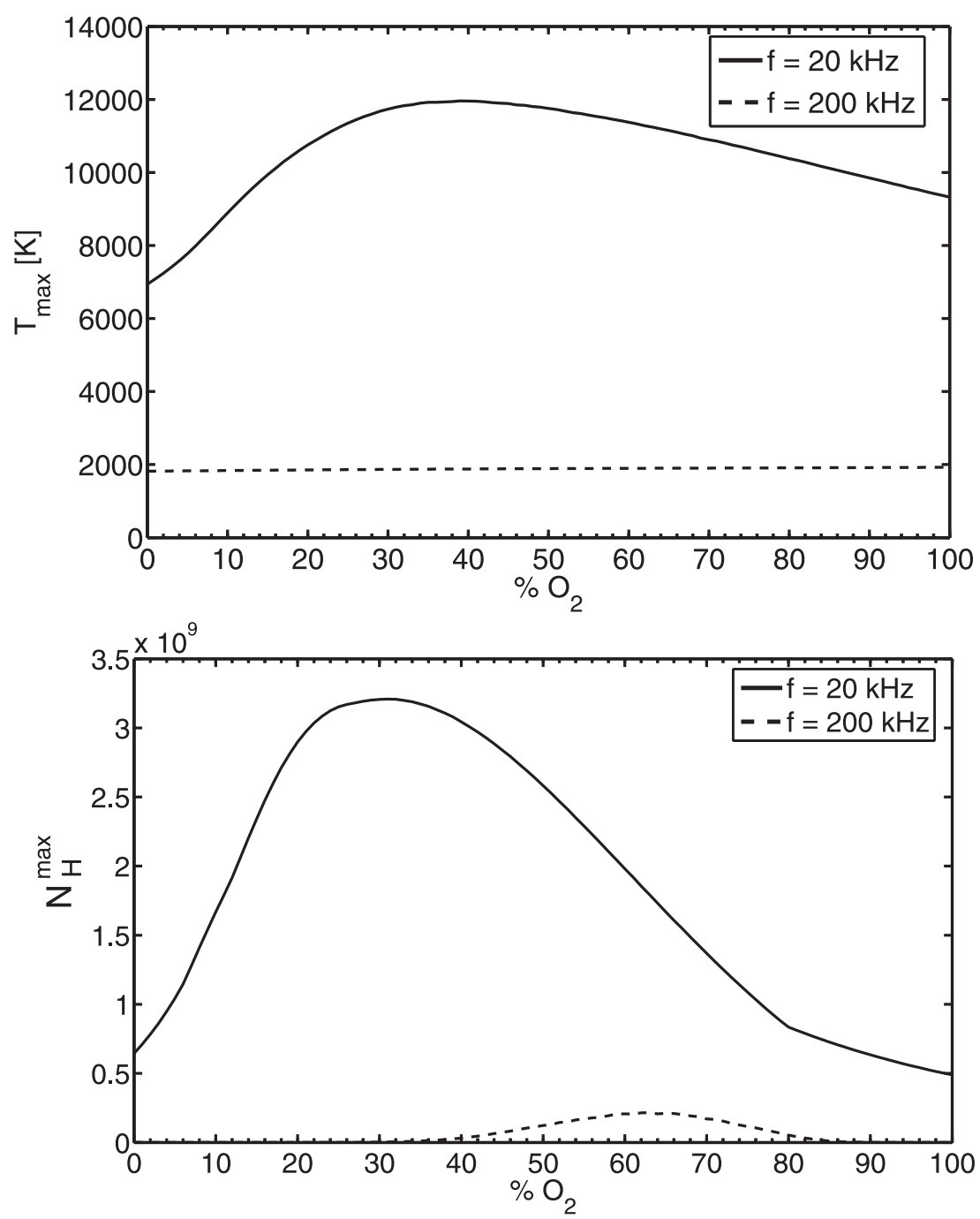

Figure 4.5: Maximum temperature (top) and number of $\mathrm{H}$ molecules (bottom) during the first cycle of a $\mathrm{H}_{2}-\mathrm{O}_{2}$ bubble, as function of the $\mathrm{O}_{2}$ percentage in the initial bubble, at $20 \mathrm{kHz}$ (solid) and $200 \mathrm{kHz}$ (dashed) $\left(R_{0}=6 \mu \mathrm{m}, P_{a}=1.4 \mathrm{~atm}, T_{\infty}=20^{\circ} \mathrm{C}\right)$. At $20 \mathrm{kHz}$, when the temperature is high enough to activate the strongly exothermal dissociating reactions of $\mathrm{H}_{2}$ and $\mathrm{O}_{2}$, the mixture with the stoichiometric composition of water $\left(2 / 3 \mathrm{H}_{2}, 1 / 3 \mathrm{O}_{2}\right)$ provides the highest temperature and radical production. This mimics the well-known explosive nature of such mixtures. 
(see Tab. 4.3.1) and we refer the reader to Ref. [42] for a more complete scheme of reactions taking place inside a bubble with this initial composition. Reactions 1 and 2 are the initiating endothermic steps. Given their high activation energies $E_{1} / k_{B}=52177 \mathrm{~K}$ and $E_{2} / k_{B}=59893 \mathrm{~K}$, it is crucial that a minimum temperature is reached (around $2000 \mathrm{~K}$ and $2200 \mathrm{~K}$ respectively). Under these values the coefficients $\exp \left(-E_{j} / k_{B} T\right)$ of Arrhenius law are too low $\left(<10^{-11}\right)$ for dissociation to take place. Conversely, when the temperatures is high enough to allow the initial dissociation, the produced $\mathrm{H}$ and $\mathrm{O}$ radicals become reactants for the propagation reactions 3-6, among which 3 and 4 are branching reactions, as they increase the chain carriers $(\mathrm{H}, \mathrm{O}$, $\mathrm{OH})$. At the same time, exothermic termination reactions 6-9 are initiated, removing chain carriers. The overall effect of the chain reaction is a strong release of energy with the consequent increase in temperature that we observe in simulations. The termination reactions have a very low inversely proportional temperature dependency, as their activation energy $E_{j} / k_{B}$ is zero and their $c_{j}$ coefficient is negative, while the initiation reactions are strongly dependent on the temperature. Therefore, when the temperature increases, the propagation reactions overcome the termination reactions, resulting into an increased number of $\mathrm{H}, \mathrm{OH}$ and $\mathrm{O}$ radicals.

This results appears particularly relevant, as it reproduces the explosive well known behavior of stoichiometric mixtures of $\mathrm{H}_{2}$ and $\mathrm{O}_{2}$, thus providing a further strong validation for the code. Moreover, it can be used in technological applications for optimization purposes. The peak value of $\mathrm{H}$ radicals follows indeed the temperature trend, having a maximum in mixtures around $34 \% \mathrm{O}_{2}$ while $\mathrm{O}$ and $\mathrm{OH}$ radical curves present a shift, with a maximum respectively at $70 \% \mathrm{O}_{2}$ and $60 \% \mathrm{O}_{2}$. Therefore we can conclude that in low frequency reactors there is an optimal range of composition for the global radical production, between $40 \%$ and $60 \% \mathrm{O}_{2}$.

\subsubsection{Effects of the driving frequency}

We examined a range of ultrasound frequencies from $7 \mathrm{kHz}$ to $200 \mathrm{kHz}$. When the ultrasound frequency increases, the peak production of all chemical species decreases, together with the maximum temperature reached during the cycle (see Fig. 4.6). In argon bubbles a maximum peak temperature appears at $15 \mathrm{kHz}$ (see Fig. 4.1). The explanation relies on the fact that, when a lower frequency is applied, the bubble has more time to grow, generating a more violent collapse afterwards. However, below a certain frequency, the longer duration of the expansion gives the bubble the time to cool and the lower inner pressure enhances water vapor entrapment. As the number of particles grows, the heat capacity raises, lowering the heat entering the bubble at the following collapse therefore the peak temperature. This phenomenon was first theoretically explained and at the same time observed experimentally as a reduction 

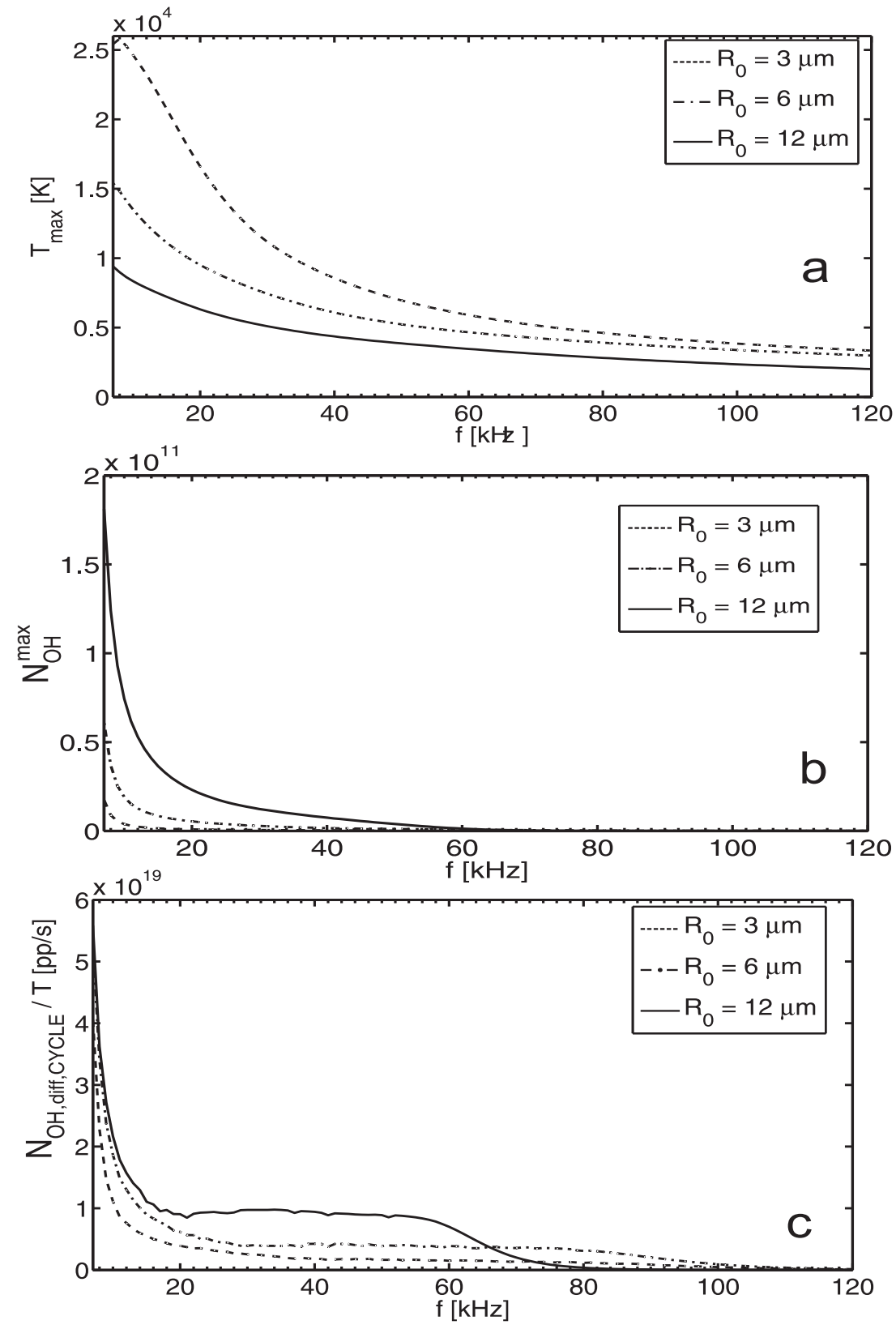

Figure 4.6: Maximum temperature (a), number of $\mathrm{OH}$ molecules (b) and $\mathrm{OH}$ diffusion rate (c) during the first oscillating cycle of an air bubble, as a function of frequency $\left(R_{0}=6 \mu \mathrm{m}, P_{a}=1.4 \mathrm{~atm}, T_{\infty}=20^{\circ} \mathrm{C}\right)$. The peak temperature and radical production decrease with the frequency, because the bubble has less time to grow and the collapse is weaker. However, the number of collapses per unit time increases with the frequency. Therefore high sonochemical rates can be achieved up to $60 \mathrm{kHz}$. 
of the emitted light in SBSL [26]. From the sonochemical point of view, the increased amount of water vapor provides a higher number of reactants for water dissociation, leading to an increased production of $\mathrm{H}, \mathrm{OH}$ and $\mathrm{O}$. Despite the behavior of air, $\mathrm{O}_{2}$, $\mathrm{H}_{2}$ and $\mathrm{N}_{2}$ seems qualitatively different, as no peak appears in the considered range of frequencies, this is not the case, as a similar peak appears at lower frequencies. We did not include those results because such frequencies are not used in sonochemical reactors, due to technological problems (temperature control, noise, etc.).

However, for a long term analysis, if we wish to compare sonochemical reactors operating at different frequencies, the relevant quantity is the sonochemical rate, i.e. the number of radicals diffusing into the liquid per unit time. This quantity will depend both on the absolute peak production at each collapse (decreasing with the frequency) and the number of collapses per unit time (increasing with the frequency). The combination of these two competing effects, leads to a maximum in radical production rate curves in certain ranges of frequencies, changing with the bubble size. As the ultimate task of our analysis is to make recommendations for multibubble reactors, we will point out the optimal frequency range considering the curves for different bubble sizes and different chemical species. For $\mathrm{O}, \mathrm{H}$ and $\mathrm{OH}$ radicals the optimal production rate is localized in the range between 7 and $60 \mathrm{kHz}$, while for $\mathrm{NO}_{x}$ it is between 20 and $40 \mathrm{kHz}$ (see Fig. 4.6). For $\mathrm{N}, \mathrm{NH}, \mathrm{NH}_{2}$ and $\mathrm{NH}_{3}$, the radical rate does not present any maximum, but monotonically decreases when the frequency increases, as the peak production does. Therefore we can conclude that the optimal operating range for such single-bubble based sonochemical reactors is between $20 \mathrm{kHz}$ and $40 \mathrm{kHz}$.

\subsubsection{Effects of the ambient radius}

We considered the range between $1 \mu \mathrm{m}$ and $12 \mu \mathrm{m}$, that is the range of interest in new generation efficient multibubble reactors of Ref. [2]. We found that, given a fixed driving pressure, the ambient radius of the most chemically active bubbles decreases when the frequency increases (see Fig. 4.7) and, given a fixed driving frequency, it decreases when the driving pressure increases 4.8. Therefore, from the technological point of view, it would be desirable to control the size of the nucleated bubbles; for eg., with reactors working at $200 \mathrm{kHz}$ the optimal size would be 6-7 $\mu \mathrm{m}$. In the case of bubbles generated from acoustically driven air pockets entrapped inside artificial crevices [2], this should be achievable by changing the dimension and the shape of the crevices themselves 


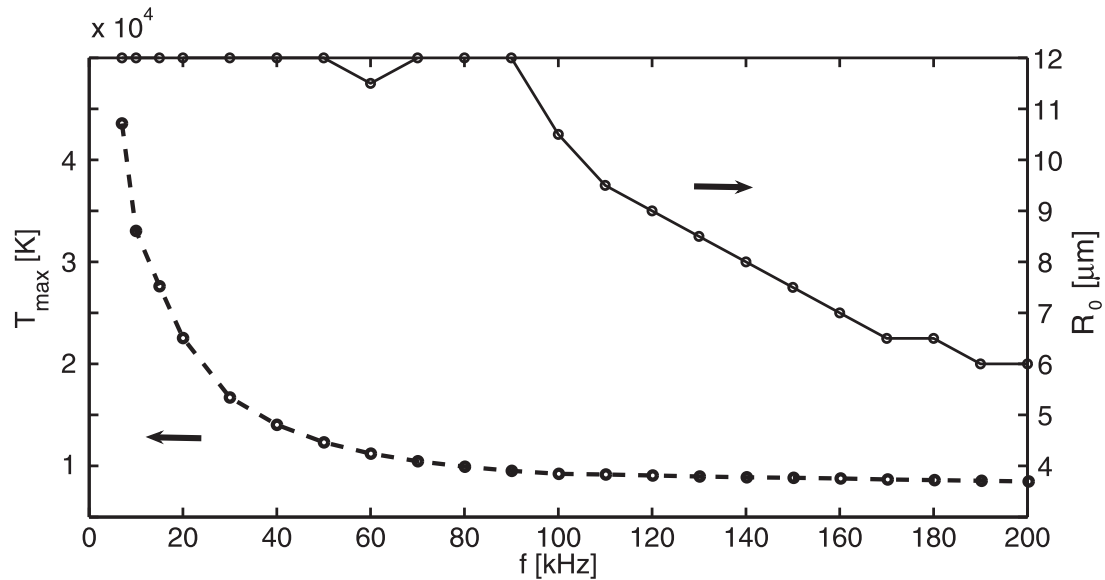

Figure 4.7: Ambient radius (solid) and temperature (dashed) corresponding to the highest $\mathrm{H}$ radicals production during the first oscillating cycle of an air bubble $\left(P_{a}=\right.$ $1.4 \mathrm{~atm}, T_{\infty}=20^{\circ} \mathrm{C}$ ). As the frequency increases, the most active bubbles become smaller.

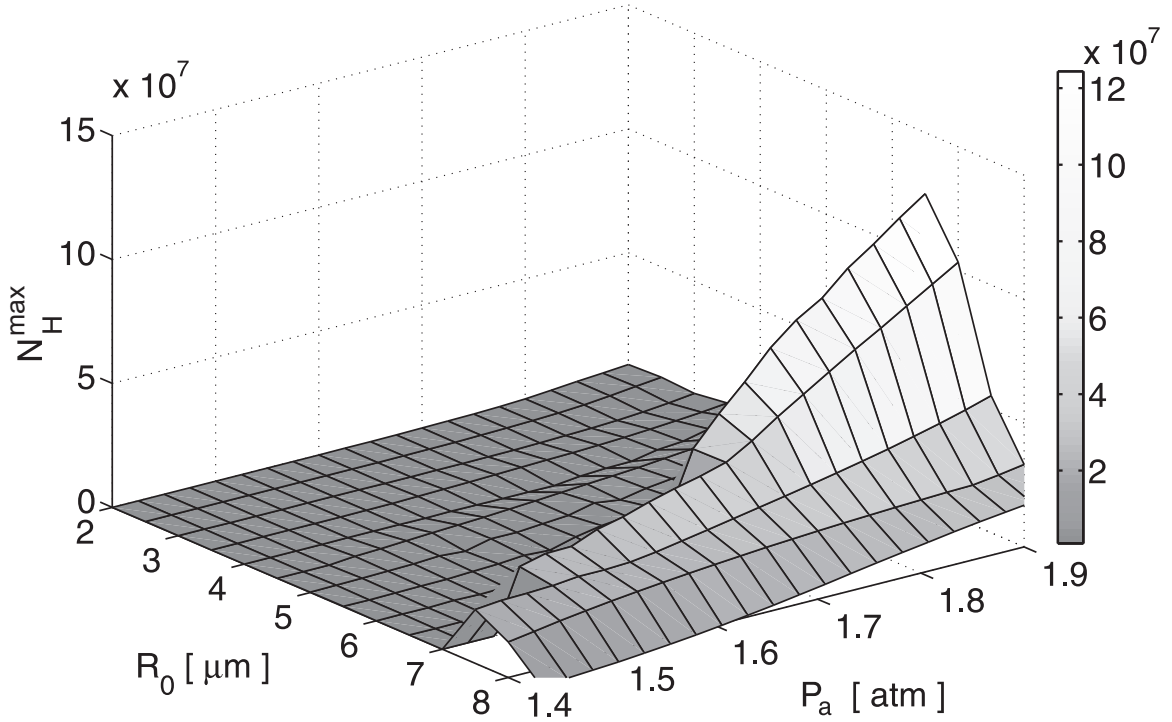

Figure 4.8: Maximum number of $\mathrm{H}$ radicals during the first oscillating cycle of an air bubble, as function of the driving pressure and the ambient radius, at $f=200 \mathrm{kHz}$. As the pressure increases, the most active bubbles become smaller. 

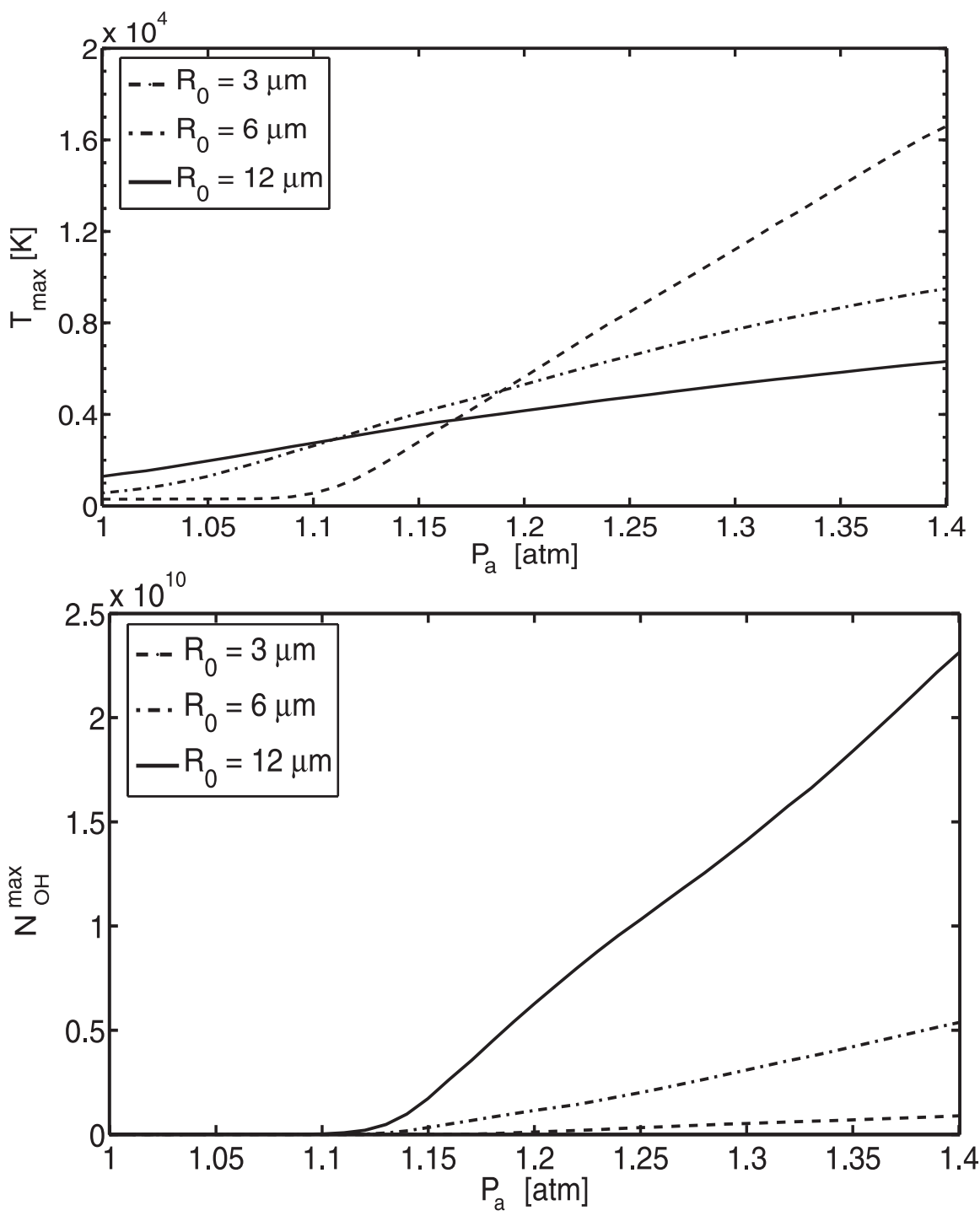

Figure 4.9: Maximum temperature (top) and number of $\mathrm{OH}$ molecules (bottom) during the first oscillating cycle of an air bubble, as function of the driving pressure ( $f$ $=20 \mathrm{kHz}, T_{\infty}=20^{\circ} \mathrm{C}$ ). The chemical production and the temperature increase with the driving. Below $1.1 \mathrm{~atm}$, corresponding to a collapse temperature of $2200 \mathrm{~K}$, no radicals are produced. 


\subsubsection{Effects of the driving pressure}

We examined a range of driving pressures from 1 to 1.4 atm both at $20 \mathrm{kHz}$ and $200 \mathrm{kHz}$. The peak production of all the chemical species increases with the driving. Under a certain pressure threshold, rising with the frequency, no radicals are produced. In air bubbles, at $20 \mathrm{kHz}$ this threshold is around $1.1 \mathrm{~atm}$, while at $200 \mathrm{kHz}$ it is around $1.3 \mathrm{~atm}$ (see Fig. 4.9). Both these conditions correspond to peak temperatures around $2200 \mathrm{~K}$. Under this value no chemical activity was recorded, because the coefficient $\exp \left(-E_{j} / k_{B} T\right)$ in Eq.(4.12) for the first reaction of water dissociation, $\mathrm{H}_{2} \mathrm{O}+\mathrm{M} \rightarrow \mathrm{H}+\mathrm{OH}+\mathrm{M}$, was too low $\left(<10^{-11}\right)$. The same trends were found in $\mathrm{O}_{2}$ bubbles, with a small shift in the pressure thresholds for sonochemical activity inception towards higher values, due to the slightly lower temperatures reached with this gas.

\subsubsection{Effects of the liquid temperature}

We examined a range of temperatures from $5^{\circ} \mathrm{C}$ to $90^{\circ} \mathrm{C}$. When the liquid temperature increases, also the pressure of the vapor inside the bubble does, while both viscosity and surface tension decrease, together with the forces they oppose to the bubble growth. Therefore the maximum radius grows bigger, more vapor enters the bubble and is entrapped at the following collapse. The higher number of water molecules reduces the temperature at collapse. Moreover the radius after the collapse remains bigger, further decreasing the maximum temperature. Conversely, the big amount of water molecules provides many reactants for water dissociation reactions. At $20 \mathrm{kHz}$, for a $3 \mu \mathrm{m}$ air bubble, the combination of these two competing factors leads to a peak in $\mathrm{H}, \mathrm{OH}$ and $\mathrm{O}$ production at $T_{0}=65^{\circ} \mathrm{C}$. These value shifts to lower ones as the bubble size increases (see Fig. 4.10). Conversely, they shift towards higher temperatures when the frequency increases. At $200 \mathrm{kHz}$, the peak for a $3 \mu \mathrm{m}$ bubble is around a liquid temperatures of $75^{\circ} \mathrm{C}$ because the time that bubbles have to entrap vapor is lower and therefore a higher temperature is requested.

We examined also $\mathrm{O}_{2}$ and argon bubbles, in order to exclude the nitrogen chemistry for understanding its role. In pure $\mathrm{O}_{2}$ bubbles, we found exactly the same trends than in air bubbles. This supports the idea that the change in radical production with the ambient temperature is not due to the nitrogen chemistry but mainly to the water vapor entrapped in the system at collapse and the chemical reactions involving it, i.e. oxygen and hydrogen chemistry.

With argon bubbles, at high frequency $(200 \mathrm{kHz})$ we found the same trends as with air bubbles, both for temperature and radical production, with a shift of the peaks towards lower liquid temperatures (between $45^{\circ} \mathrm{C}$ and $60^{\circ} \mathrm{C}$ for a $3 \mu \mathrm{m}$ bub- 

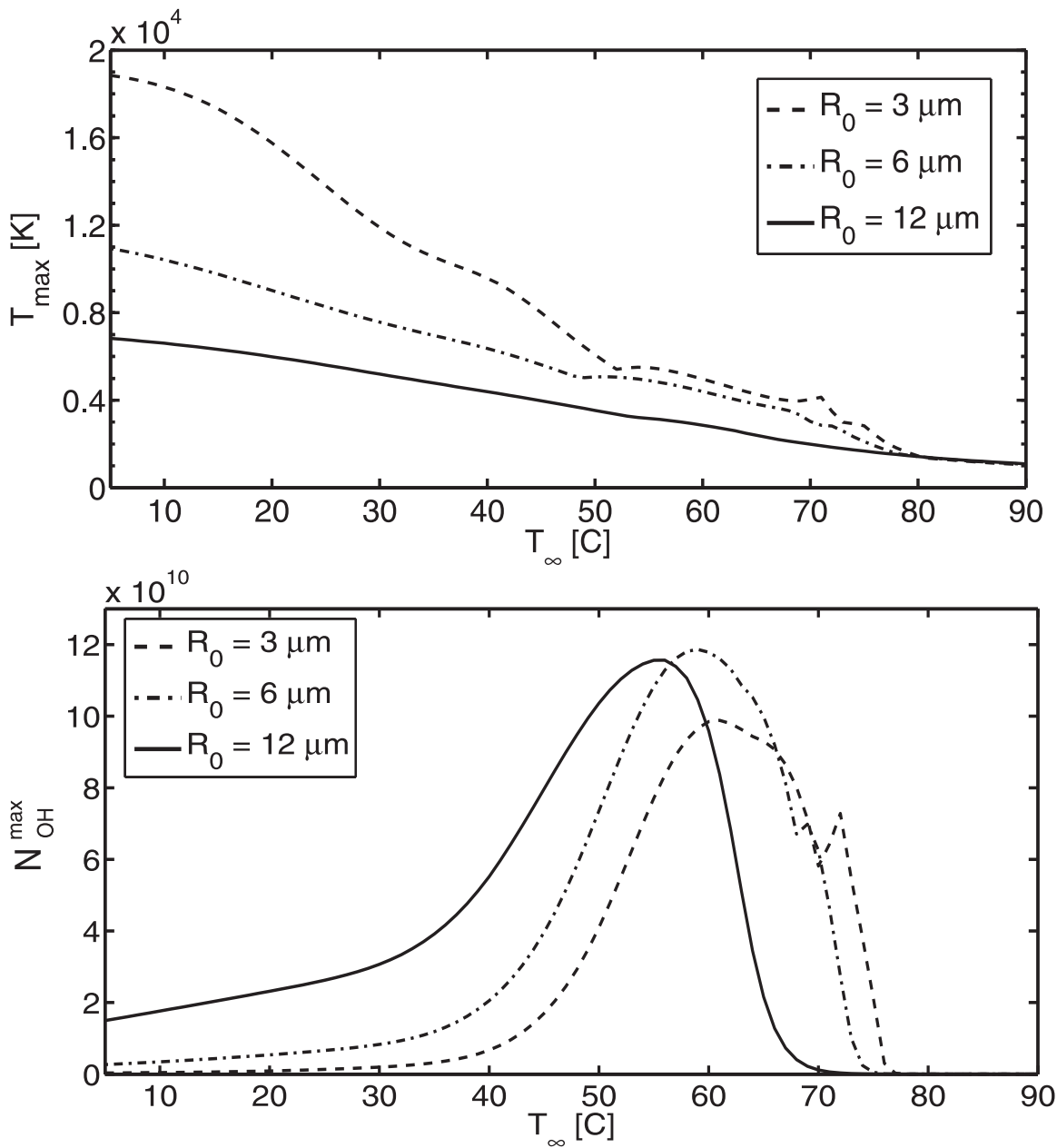

Figure 4.10: Maximum temperature (top), and number of $\mathrm{OH}$ molecules (bottom) during the first oscillating cycle of an air bubble, as a function of the liquid temperature $\left(f=20 \mathrm{kHz}, P_{a}=1.4 \mathrm{~atm}\right)$. As the liquid temperature increases, more vapor enters the bubble, lowering the collapse temperature but providing a higher number of reactants for water dissociation. The combination of these two competing effects gives an optimal liquid temperature around $60^{\circ} \mathrm{C}$. 

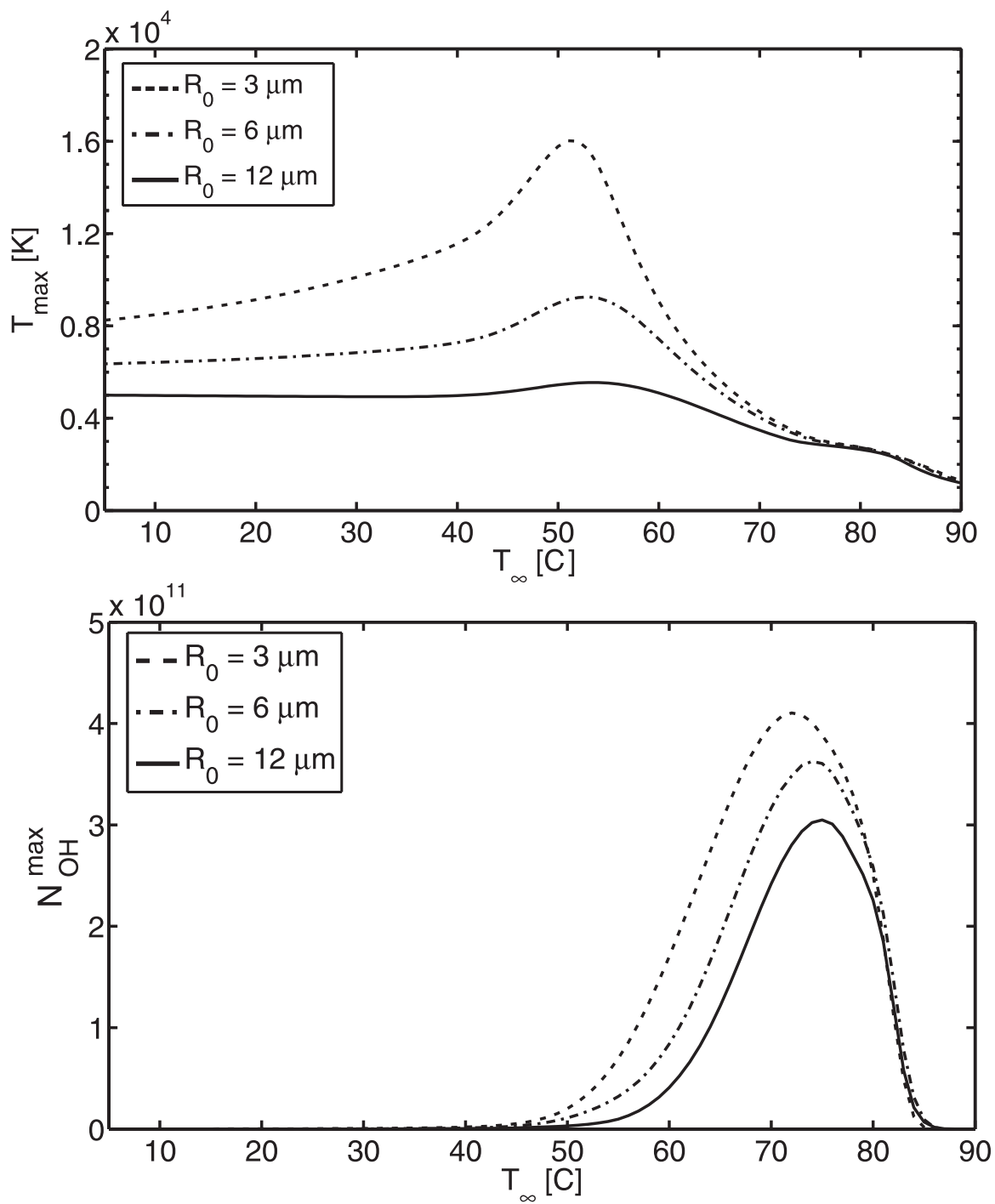

Figure 4.11: Maximum temperature (top), and number of $\mathrm{OH}$ molecules (bottom) during the first oscillating cycle of an argon bubble, as a function of the liquid temperature $\left(f=20 \mathrm{kHz}, P_{a}=1.4 \mathrm{~atm}\right)$. As the liquid temperature increases, more vapor enters the bubble, providing a higher number of reactants for water dissociation. However the bubble temperature does not decrease, due to the activation of $\mathrm{H}_{2}$ and $\mathrm{O}_{2}$ strongly exothermal dissociating reactions. The combination of these effects gives an optimal liquid temperature around $70^{\circ} \mathrm{C}$. 
ble). As the temperature reached inside the bubble is higher with argon than with air bubbles, more heat is available for evaporating the surrounding water and therefore lower liquid temperatures are required. However, at low frequency $(20 \mathrm{kHz})$, when the temperature at collapse rises over $7000 \mathrm{~K}$, a completely different thermal behavior appears with argon bubbles as compared to air and $\mathrm{O}_{2}$ bubbles: the bubble temperature presents a maximum when the liquid temperature is around $50^{\circ} \mathrm{C}$. Consequently, also the chemical peaks are shifted towards higher liquid temperatures (between $60^{\circ} \mathrm{C}$ and $80^{\circ} \mathrm{C}$ for a $3 \mu \mathrm{m}$ bubble) (see Fig. 4.11). In order to understand this unexpected behavior, we artificially turned off, one by one, all the chemical reactions and we found that this temperature peak is due to the highly exothermal hydrogen and oxygen chain reactions of the kind described in Tab. 4.3.1. In particular, these reactions become important when the temperature is enough high to activate the initiating $\mathrm{H}_{2}$ and $\mathrm{O}_{2}$ dissociating reactions (reaction 1 and 2 in Tab.4.3.1) and when the number of reactants is enough high and close to the stochiometric composition to allow the chain propagation.

As the diffusivity of water inside argon is much lower than in air, a higher amount of water molecules are entrapped inside the argon bubble. Below a certain threshold of entrapped vapor, this turns into lower temperature as compared to air and $\mathrm{O}_{2}$. When the liquid temperature rises and so does the amount of the vapor, the number of $\mathrm{H}$ and $\mathrm{OH}$ radicals produced and recombining into $\mathrm{H}_{2}$ and $\mathrm{O}_{2}$ at low temperature becomes relevant. When they are newly dissociated due to the high temperature, they give origin to exothermal chain reactions thus abruptly increasing the peak temperature. Once again the effect of exothermal hydrogen and oxygen chain reactions appears crucial to understand the thermal behavior of the bubbles and its chemical feedback, when the temperature is high enough to activate them, i.e. at low frequency.

For technological applications, we can conclude that, at high frequency $(200 \mathrm{kHz})$, the optimal range of the liquid temperature is between $60^{\circ} \mathrm{C}$ and $80^{\circ} \mathrm{C}$ for pure air, $40^{\circ} \mathrm{C}$ and $60^{\circ} \mathrm{C}$ for pure argon, thus around $60^{\circ} \mathrm{C}$ for argon - air mixtures. At low frequency $(20 \mathrm{kHz})$, the optimal range is between $50^{\circ} \mathrm{C}$ and $70^{\circ} \mathrm{C}$ for pure air and from $60^{\circ} \mathrm{C}$ and $80^{\circ} \mathrm{C}$ for pure argon, therefore between $60^{\circ} \mathrm{C}$ and $70^{\circ} \mathrm{C}$ for argonair mixtures. In both cases a liquid temperature around $60^{\circ} \mathrm{C}$ can enhance chemical production.

\subsection{Summary and conclusions}

We examined the radical production during the early transient of a single cavitation bubble. This choice was made in order to match the operating conditions of a new 
generation of sonochemical reactors [2], where the bubble life is of few acoustic cycles. We wanted both to understand the mechanisms leading to radical formation and to provide optimal operating conditions for technological applications.

We found that the chemical production is strongly affected by two factors: the gas temperature and the composition of the bubble at collapse. Below $2200 \mathrm{~K}$, there are hardly any chemical reactions taking place. Between $2200 \mathrm{~K}$ and $7000 \mathrm{~K}$, the most relevant factor is the temperature, while above $7000 \mathrm{~K}$ it is the composition of the bubble at collapse. In particular, in this temperature range, we underlined the importance of water vapor, $\mathrm{H}_{2}$ and $\mathrm{O}_{2}$ dissociating reactions, that all have feedback on the temperature. The dissociation of water is a strongly endothermal process and reduces the temperature of the bubble, while the dissociation of $\mathrm{H}_{2}$ and $\mathrm{O}_{2}$ are the initiating steps of globally strongly exothermal chain reactions that increase abruptly the temperature, thus enhancing, in return, other chemical reactions. We found that mixtures of $\mathrm{H}_{2}$ and $\mathrm{O}_{2}$ with the stoichiometric composition of water give origin to a peak in temperature respect to the other compositions, whenever the temperatures are high enough for $\mathrm{H}_{2}$ and $\mathrm{O}_{2}$ dissociation to take place thus initiating the exothermal chain reactions (low frequency). This reproduces the well known chemical explosive behavior of such mixtures and provides a strong evidence of the reliability of the adopted model.

In sonochemical reactors operating at low frequency (below $20 \mathrm{kHz}$ ), where the maximum temperatures at collapse are above $7000 \mathrm{~K}$, a good strategy to enhance the radical production is to use such mixtures of $\mathrm{H}_{2}$ and $\mathrm{O}_{2}$. Conversely, in high frequency reactors (around $200 \mathrm{kHz}$ ), where the maximum reached temperatures are below $7000 \mathrm{~K}$, a good strategy to enhance the radical production is to add a large amount of argon (around 90\%), thus increasing the polytropic exponent of the mixture and therefore the peak temperature.

From the study of the size of the most active bubbles, we discovered that an other strategy could be to promote the nucleation of big bubbles in low frequency reactors and small bubbles in high frequency reactors, for example by changing the size of the pits from where the bubbles detach in reactors based on heterogeneous nucleation from artificial crevices [2].

We also studied the effects of the driving pressure, the frequency and the liquid temperature. We found that higher driving pressures correspond to higher temperature and radical production, while higher frequencies correspond to lower temperature at collapse and therefore lower radical production. The reason of this behavior is that, when the frequency increases, the bubble has less time to grow, so the collapse is less violent. However, the definition of sonochemical efficiency requires the evaluation of the radical diffusion rate, i.e. the number of radicals diffusing into the liquid 
per unit time, in order to optimize the frequency. When we increase the frequency, competing effects appear between the lower production per collapse and the higher number of collapses per unit time. The combination of these two elements leads to an optimal operating range between $20 \mathrm{kHz}$ and $40 \mathrm{kHz}$.

For what concerns the optimal conditions of the liquid, competing effects appear as well. When we raise the liquid temperature, the amount of water vapor entrapped in the bubble at collapse increases. On one hand, this reduces the peak temperature, because of the highly endothermic $\mathrm{H}_{2} \mathrm{O}$ dissociating reactions and the lower polytropic exponent of the water vapor as compared to diatomic and monatomic gases. On the other hand, the amount of reactants for water dissociation is also increased. At high frequency $(200 \mathrm{kHz})$, where collapse temperatures are below $3000 \mathrm{~K}$, the combination of these two competing effects results into an optimal liquid temperature around $50^{\circ} \mathrm{C}-70^{\circ} \mathrm{C}$ for air and oxygen bubbles, shifted to $60^{\circ} \mathrm{C}-80^{\circ} \mathrm{C}$ for argon bubbles. At low frequency $(20 \mathrm{kHz})$, where temperatures are higher than $7000 \mathrm{~K}$, a third factor comes into play, namely the effect of exothermal $\mathrm{H}_{2}$ and $\mathrm{O}_{2}$ dissociating reactions, particularly evident in argon bubbles because of the higher amount of entrapped water as compared to air bubbles. As a general indication, heating a sonochemical reactor to around $60^{\circ} \mathrm{C}$ should increase its radical production.

The present study could provide good indications to tune the working conditions of a sonochemical reactor of the kind presented in Ref. [2] in order to enhance its efficiency. 


\section{References}

[1] L. Stricker, A. Prosperetti, and D. Lohse, "Validation of an approximate model for the thermal behavior in acoustically driven bubbles", J. Acoust. Soc. Am. 130, 3243-3251 (2011).

[2] D. Fernández Rivas, L. Stricker, A. Zijlstra, H. Gardeniers, D. Lohse, and A. Prosperetti, "Ultrasound artificially nucleated bubbles and their sonochemical radical production”, Ultrason. Sonochem. 20, 510-524 (2013).

[3] D. F. Gaitan, L. A. Crum, C. C. Church, and R. A. Roy, "Sonoluminescence and bubble dynamics for a single, stable, cavitation bubble", J. Acoust. Soc. Am. 91, 3166-3183 (1992).

[4] L. A. Crum, "Sonoluminescence”, Phys. Today 47, 22-29 (1994).

[5] B. P. Barber, R. A. Hiller, R. Löfstedt, S. J. Putterman, and K. R. Weninger, "Defining the unknowns of sonoluminescence", Phys. Rep. 281, 65-143 (1997).

[6] T. J. Matula, "Inertial cavitation and single-bubble sonoluminescence", Philos. Trans. R. Soc. London, Ser. A 357, 225-249 (1999).

[7] M. P. Brenner, S. Hilgenfeldt, and D. Lohse, "Single bubble sonoluminescence", Rev. Mod. Phys. 74, 425-484 (2002).

[8] K. S. Suslick, "Sonochemistry”, Science 247, 1439-1445 (1990).

[9] K. S. Suslick, S. J. Doktycz, and E. B. Flint, "On the origin of sonoluminescence and sonochemistry”, Ultrasonics 28, 280-290 (1990).

[10] K. S. Suslick and G. J. Price, "Applications of ultrasound to materials chemistry”, Ann. Rev. Mat. Sci. 29, 295-326 (1999).

[11] L. A. Crum, T. J. Mason, J. L. Reisse, and K. S. Suslick, eds., See the articles in Sonochemistry and Sonoluminescence (Kluwer Academic Publishers, Dordrecht) (1999).

[12] T. J. Mason and J. P. Lorimer, Applied sonochemistry, the uses of power ultrasound in chemistry and processing (Wiley-VCH, Weinheim) (2002).

[13] K. S. Suslick and D. J. Flannigan, "Inside a collapsing bubble: Sonoluminescence and the conditions during cavitation", Ann. Rev. Phys. Chem. 59, 659683 (2008). 
[14] D. Lohse, M. P. Brenner, T. F. Dupont, S. Hilgenfeldt, and B. Johnston, "Sonoluminescing air bubbles rectify argon”, Phys. Rev. Lett. 78, 1359-1362 (1997).

[15] D. Lohse and S. Hilgenfeldt, "Inert gas accumulation in sonoluminescing bubbles”, J. Chem. Phys. 107, 6986-6997 (1997).

[16] T. J. Mason, J. P. Lorimer, D. M. Bates, and Y. Zhao, "Dosimetry in sonochemistry: the use of aqueous terephthalate ion as a fluorescence monitor", Ultrason. Sonochem. 1, S91-S95 (1994).

[17] T. Mason, A. Cobley, J. Graves, and D. Morgan, "New evidence for the inverse dependence of mechanical and chemical effects on the frequency of ultrasound", Ultrason. Sonochem. 18, 226-230 (2011).

[18] G. Mark, A. Tauber, R. Laupert, H.-P. Schuchmann, D. Schulz, A. Mues, and C. von Sonntag, "OH-radical formation by ultrasound in aqueous solution - Part II: Terephthalate and Fricke dosimetry and the influence of various conditions on the sonolytic yielde", Ultrason. Sonochem. 5, 41-52 (1998).

[19] M. A. Beckett and I. Hua, "Impact of ultrasonic frequency on aqueous sonoluminescence and sonochemistry”, J. Phys. Chem. A 105, 3796-3802 (2001).

[20] S. Hatanaka, "Relationship between radical production and cavitation dynamics of a sonoluminescing bubble", in 8th International Symposium on Cavitation Proceedings, 242 (2012).

[21] K. Yasui, "Influence of ultrasonic frequency on multibubble sonoluminescence”, J. Acoust. Soc. Am. 112, 1405-1413 (2002).

[22] R. Toegel, S. Hilgenfeldt, and D. Lohse, "The effect of surfactants on single bubble sonoluminescence", Phys. Rev. Lett. 84, 2509-2512 (2000).

[23] G. Price, M. Ashokkumar, and F. Grieser, "Sonoluminescence quenching of organic compounds in aqueous solution: Frequency effects and implications for sonochemistry", J. Am. Chem. Soc. 126, 2755-2762 (2004).

[24] S. Sochard, A. Wilhelm, and H. Delmas, "Modelling of free radicals production in a collapsing gas-vapour bubble", Ultrason. Sonochem. 4, 77-84 (1997).

[25] B. D. Storey and A. J. Szeri, "A reduced model of cavitation physics for use in sonochemistry”, Proc. Roy. Soc. London A 457, 1685-1700 (2001). 
[26] R. Toegel, B. Gompf, R. Pecha, and D. Lohse, "Does water vapor prevent upscaling sonoluminescence?”, Phys. Rev. Lett. 85, 3165-3168 (2000).

[27] T. J. Mason, J. P. Lorimer, and D. M. Bates, "Quantifying sonochemistry: casting some light on a 'black art"', Ultrasonics 30, 40-42 (1992).

[28] D. N. Rassokhin, G. V. Kovalev, and L. T. Bugaenko, “Temperature effect on the sonolysis of methan/water mixtures”, J. Am. Chem. SOC 117, 344-347 (1995).

[29] T. Tuziuti, S. Hatanaka, K. Yasui, T. Kozuka, and H. Mitome, "Effect of ambient-pressure reduction on multibubble sonochemiluminescence", J. Chem. Phys. 16, 6221-6227 (2002).

[30] M. A. Henglein, "Sonochemistry and sonoluminescence: Effects of external pressure", J. Phys. Chem. 97, 158-162 (1993).

[31] K. Yasui, T. Tuziuti, Y. Iida, and H. Mitome, "Theoretical study of the ambientpressure dependence of sonochemical reactions”, J. Chem. Phys. 119, 347-356 (2003).

[32] K. Yasui, T. Tuziuti, and Y. Iida, "Optimum bubble temperature for the sonochemical production of oxidants”, Ultrasonics 42, 579-584 (2004).

[33] F. R. Young, "Sonoluminescence from water containing dissolved gases", J. Acoust. Soc. Am. 60, 100-104 (1976).

[34] T. Kondo, J. Gamson, J. Mitchell, and P. Riesz, "Free radical formation and cell lysis induced by ultrasound in the presence of different rare gases", Int.J.Radiat.Biol. 54, 955-962 (1988).

[35] C. Petrier, A. Jeunet, J. L. Luche, and G. Reverdy, "Unexpected frequency effects on the rate of oxidative processes induced by ultrasound", J. Am. Chem. Soc. 14, 3148-3150 (1992).

[36] Y. T. Didenko and K. S. Suslick, "The energy efficiency of formation of photons, radicals and ions during single-bubble cavitation", Nature 418, 394-397 (2002).

[37] S. Koda, K.Tanaka, H. Sakamoto, T. Matsuoka, and H. Nomura, "Sonochemical efficiency during single-bubble cavitation in water", J. Phys. Chem. 108, 11609-11612 (2004).

[38] K. Yasui, T. Tuziuti, M. Sivakumar, and Y. Iida, "Theoretical study of singlebubble sonochemistry", J. Chem. Phys. 122, 224706 (2005). 
[39] V. Kamath, A. Prosperetti, and F. N. Egolfopoulos, "A theoretical study of sonoluminescence", J. Acoust. Soc. Am. 94, 248-260 (1993).

[40] K. Yasui, T. Tuziuti, T. Kozuka, A. Towata, and Y. Iida, "Relationship between the bubble temperature and main oxidant created inside an air bubble under ultrasound", J. Chem. Phys. 127, 154502 (2007).

[41] R. Toegel, S. Hilgenfeldt, and D. Lohse, "Suppressing dissociation in sonoluminescing bubbles: The effect of excluded volume", Phys. Rev. Lett. 88, 034301 (2002).

[42] R. Toegel and D. Lohse, "Phase diagrams for sonoluminescing bubbles: A comparison between experiment and theory", J. Chem. Phys. 118, 1863 (2003).

[43] B. D. Storey and A. J. Szeri, "Water vapour, sonoluminescence and sonochemistry”, Proc. Roy. Soc. London A 456, 1685-1709 (2000).

[44] S. Hilgenfeldt and D. Lohse, "Predictions for upscaling sonoluminescence", Phys. Rev. Lett. 82, 1036-1039 (1999).

[45] S. Hilgenfeldt, S. Grossmann, and D. Lohse, "A simple explanation of light emission in sonoluminescence", Nature 398, 402-405 (1999). 


\section{5 \\ Bubble growth by gas diffusion *}

Given the importance of diffusive phenomena when one wishes to study bubble dynamics and sonochemistry, as a first step to include these phenomena in a full PDE model, we developed a model to track the evolution of a bubble growing purely by mass diffusion. We validated the code against the analytic solution and also against experimental results, for the case of a bubble growing next to a wall. To this aim, we introduced a geometric correction to the model, in order to keep into account the disruption of the boundary layer due to the presence of the wall, and we found good agreement with the experimental data.

\subsection{Model}

We consider a spherical bubble of gas immersed inside an infinite liquid medium. The radial motion of the bubble is described by the Rayleigh-Plesset equation:

$$
\left(1-\frac{\dot{R}}{c_{L}}\right) R \ddot{R}+\frac{3}{2}\left(1-\frac{\dot{R}}{3 c_{L}}\right) \dot{R}^{2}=\frac{1}{\rho_{L}}\left(1+\frac{\dot{R}}{c_{L}}+\frac{R}{c_{L}} \frac{d}{d t}\right)\left[p-p_{\infty}-4 \mu_{L} \frac{\dot{R}}{R}-\frac{2 \sigma}{R}\right] .
$$

Here time derivatives are denoted by a dot, $R$ is the bubble radius, $c_{L}$ and $\rho_{L}$ are the speed of sound and the density of the liquid, $\mu_{L}$ is the dynamic viscosity of the liquid,

\footnotetext{
*The experimental data present in this chapter are entirely due to Oscar R. Enriquez Paz Y Puente.
} 
$\sigma$ is the surface tension coefficient, $p_{\infty}$ is the liquid ambient pressure and $p$ is the gas pressure inside the bubble. We neglect the contribution of the vapor and the total pressure inside the bubble $p$ is regarded as spatially uniform, which is acceptable for small Mach numbers. The gas inside the bubble is considered as a perfect gas and viscous dissipations inside the bubble are neglected

In order to derive an equation for the pressure, we follow the procedure adopted in Refs. [1]. We write the enthalpy equation for the gas inside the bubble in the standard form [2]:

$$
\frac{\partial}{\partial t}(\rho h-p)+\nabla \cdot(\rho h \mathbf{v})=-\nabla \cdot \mathbf{q},
$$

where $\rho$ is the density of the gas, $h$ is its enthalpy, $\mathbf{v}$ is its velocity field and $\mathbf{q}$ is the heat flux vector from the bubble to the liquid. The perfect gas law equation holds, therefore

$$
\rho h-p=\frac{1}{\gamma-1} p,
$$

in which $\gamma$ is the adiabatic index. By substituting (5.3) inside (5.2) we get

$$
\dot{p}=-(\gamma-1) \nabla \cdot[\rho h \mathbf{v}+\mathbf{q}] .
$$

Upon integration over the radial coordinate $r$, we find

$$
v=-\frac{1}{\gamma p}\left[(\gamma-1) q+\frac{1}{3} r \dot{p}\right],
$$

where $q$ and $v$ are the radial components of $\mathbf{q}$ and $\mathbf{v}$ respectively. By evaluating (5.5) at the bubble wall $r=R$, we retrieve [1]:

$$
\dot{p}=-\frac{3}{R}[\gamma p v+(\gamma-1) q]_{r=R}
$$

Unlike in Ref. [1], here we cannot assess that $v(R, t)=\dot{R}$, because a gas flux is present. The rate of change of the mass of gas inside the bubble $m_{G}$ is given by

$$
\dot{m}_{G}=4 \pi R^{2} \rho[\dot{R}-v(R, t)],
$$

therefore the velocity of the gas at the bubble wall can be expressed as

$$
\left.v\right|_{r=R}=\dot{R}-\frac{\dot{m}_{G}}{4 \pi R^{2} \rho} .
$$

We substitute it inside (5.5) and use the perfect gas law

$$
p=\rho \mathscr{R} T,
$$


with $\mathscr{R}$ the universal gas constant divided by the molecular weight of the gas and $T$ the temperature of the gas, thus getting

$$
\dot{p}=-\frac{3}{R}\left[\gamma\left(-\frac{\mathscr{R} T_{\infty} \dot{m}_{G}}{4 \pi R^{2}}+p \dot{R}\right)+(\gamma-1) q\right]_{r=R} .
$$

The mass growth of the bubble is given by the concentration gradient at the interface,

$$
\dot{m}_{G}=\left.4 \pi R^{2} D \frac{\partial c}{\partial r}\right|_{r=R}
$$

in which $c(r, t)$ is the gas concentration in the liquid, expressed in $\mathrm{kg} / \mathrm{m}^{3}$ and $D$ is the mass diffusivity of the gas inside the liquid. The heat flux at the bubble wall can be expressed as

$$
\left.q\right|_{r=R}=-\left.k \frac{\partial T}{\partial r}\right|_{r=R},
$$

where $k=k(T)$ is the gas thermal conductivity. Upon substitution of (6.15) and (5.12) inside (5.10), we find the equation for the evolution of the gas pressure:

$$
\dot{p}=\frac{3}{R}\left[\left.(\gamma-1) k \frac{\partial T}{\partial r}\right|_{R}+\gamma\left(-p \dot{R}+\left.\mathscr{R} T_{\infty} D \frac{\partial c}{\partial r}\right|_{r=R}\right)\right],
$$

The temperature distribution inside the bubble is given by the energy equation (see Chap. 2)

$$
\frac{\gamma}{\gamma-1} \frac{p}{T}\left[\frac{\partial T}{\partial t}+\frac{1}{\gamma p}\left((\gamma-1) k \frac{\partial T}{\partial r}-\frac{1}{3} r \dot{p}\right) \frac{\partial T}{\partial r}\right]-\dot{p}=\nabla \cdot(k \nabla T) .
$$

This equation has to be solved imposing the two boundary conditions that the temperature is regular at the bubble center and it remains undisturbed, equal to the temperature of the liquid, $T_{\infty}$ at the bubble wall:

$$
\begin{gathered}
\left.\frac{\partial T}{\partial r}\right|_{r=0}=0, \\
\left.T\right|_{r=R(t)}=T_{\infty} .
\end{gathered}
$$

The transport of dissolved gas in the liquid is described by the standard constantproperties convection-diffusion equation:

$$
\frac{\partial c}{\partial t}+\frac{R^{2} \dot{R}}{r^{2}} \frac{\partial c}{\partial r}=D \nabla^{2} c
$$


This equation is solved subject to Henry's law at the bubble wall:

$$
c(R, t)=H p(R, t)
$$

where $\mathrm{H}$ is the Henry's constant. For $\mathrm{CO}_{2}$ at $20^{\circ} \mathrm{C}$ in water, $\mathrm{H}=1.517 \times 10^{-5}$ $\mathrm{kg} /\left(\mathrm{m}^{3} \mathrm{~Pa}\right)$. Far from the bubble, the gas concentration is assumed to remain undisturbed at the initial value $c_{\infty}$. The following boundary condition is adopted:

$$
\frac{\partial c}{\partial r}(\infty, t)=0
$$

For the solution of the heat and gas diffusion equations (5.14), (6.13) we express both $T(r, t)$ and $c(r, t)$ as truncated Chebyshev series

$$
\begin{gathered}
\frac{T}{T_{\infty}} \approx \sum_{k=0}^{M} a_{k} T_{2 k}, \\
\frac{c}{c_{\infty}} \approx \sum_{k=0}^{N} b_{k} T_{2 k},
\end{gathered}
$$

and we use the same method described in Chap. 2 and in Ref. [3], i.e. a pseudospectral collocation method. For details the reader is referred to that chapter.

\subsection{Validation: analytical test case}

In order to validate the present model, we derive the analytical solution of the diffusion equation in the liquid, outside of a sphere with constant radius $R$ :

$$
\frac{\partial c}{\partial t}=D \nabla^{2} c
$$

valid for $R<r<\infty$. At $t=0$, an initial concentration field $c(r, 0)=c_{0}(r)$ is prescribed. At the surface of the sphere, the boundary condition $c(R, t)=c_{s}(t)$ holds. Assuming shperical symmetry, we recast (5.22) as

$$
\partial_{t}(r c)=D \partial_{r}^{2}(r c)
$$

and we perform the Laplace transform, finding

$$
\partial_{r}^{2}(r \hat{c})-\frac{s}{D}(r \hat{c})=-\frac{r c_{0}(r)}{D}
$$


in which $s$ is the Laplace variable and $\hat{c}$ is the Laplace transform of $c$. By means of the variation of parameters method [4] eq. (5.24) can be solved to find

$$
\begin{aligned}
\hat{c}=( & \left.\alpha_{1}+\frac{1}{2} \sqrt{\frac{D}{s}} \int_{R}^{r} \frac{x c_{0}(x)}{D} e^{x \sqrt{s / D}} d x\right) \frac{e^{-r \sqrt{s / D}}}{r} \\
& +\left(\alpha_{2}-\frac{1}{2} \sqrt{\frac{D}{s}} \int_{\infty}^{r} \frac{x c_{0}(x)}{D} e^{-x \sqrt{s / D}} d x\right) \frac{e^{r \sqrt{s / D}}}{r} .
\end{aligned}
$$

Here $x$ is an integration variable, $\alpha_{1}$ and $\alpha_{2}$ are two constants, to be determined by substituting in (5.25) the transformed boundary conditions

$$
\begin{gathered}
\hat{c}(R, s)=\hat{c}_{s}(s), \\
\hat{c}(r \rightarrow \infty, s)=c_{\infty} / s .
\end{gathered}
$$

The solution is

$$
\begin{aligned}
\hat{c}=\frac{R \hat{c}_{s}}{r} e^{-\sqrt{s / D}(r-R)}+\frac{1}{2 r \sqrt{D s}}[ & \int_{R}^{\infty} x e^{-\sqrt{s / D}|r-x|} c_{0}(x) d x \\
& \left.-e^{-\sqrt{s / D}(r-R)} \int_{R}^{\infty} x e^{-\sqrt{s / D}(x-R)} c_{0}(x) d x\right] .
\end{aligned}
$$

After inverting the transform, an expression for $c(r, t)$ is found:

$$
\begin{aligned}
c(r, t)=\frac{R(r-R)}{2 r \sqrt{\pi D}} & \int_{0}^{t} c_{s}(t-\tau) \exp \left[-\frac{(r-R)^{2}}{4 D \tau}\right] \tau^{-3 / 2} d \tau \\
+ & \frac{1}{2 r \sqrt{\pi D t}}\left\{\int_{R}^{\infty} x|r-x| c_{0}(x) \exp \left[-\frac{(r-R)^{2}}{4 D t}\right] d x\right. \\
& \left.-\int_{R}^{\infty} x(x+r-2 R) c_{0}(x) \exp \left[-\frac{(x+r-2 R)^{2}}{4 D t}\right] d x\right\} .
\end{aligned}
$$

By substituting into (5.29) a constant value as initial and boundary condition $c_{0}(r)=$ $c_{s}(t)=C_{0}$, a constant expression is retrieved: $c(r, t)=C_{0}$, as expected.

The correct time scale to use for the non-dimensionalization of the problem is given by the analysis of the mass flux entering the bubble:

$$
\frac{d}{d t}\left(\frac{4}{3} \pi R^{3} \rho_{G}\right) \simeq 4 \pi R^{2} D \frac{\left.c\right|_{R}-c_{\infty}}{\delta},
$$

in which $\delta$ is the thickness of the boundary layer evaluated as $\delta=\sqrt{D t}$. By assumption that $\rho_{G}$ remains approximately constant, i.e. neglecting surface tension overpressure, we find

$$
\rho_{G} \frac{d R}{d t} \simeq \sqrt{\frac{D}{t}}\left(\left.c\right|_{R}-c_{\infty}\right) .
$$


By integration from $t=0$ to $\infty$, assuming that $\left.c\right|_{R}$ remains constant, i.e. neglecting again surface tension, the following expression is retrieved:

$$
R(t) \simeq R_{0}-2 \sqrt{D t} \frac{c_{\infty}-\left.c\right|_{R}}{\rho_{G}}
$$

The total dissolution time $t_{\text {diss }}$, which is also the time scale for the problem, is found by setting $R=0$

$$
t_{d i s s} \simeq \frac{1}{4}\left(\frac{\rho_{G}}{c_{\infty}-\left.c\right|_{R}}\right)^{2} \frac{R_{0}^{2}}{D}
$$

In order to validate the code, we considered a specific test case:

$$
\begin{aligned}
& c_{S}(t)=C+B t \\
& c(r, 0)=C R / r
\end{aligned}
$$

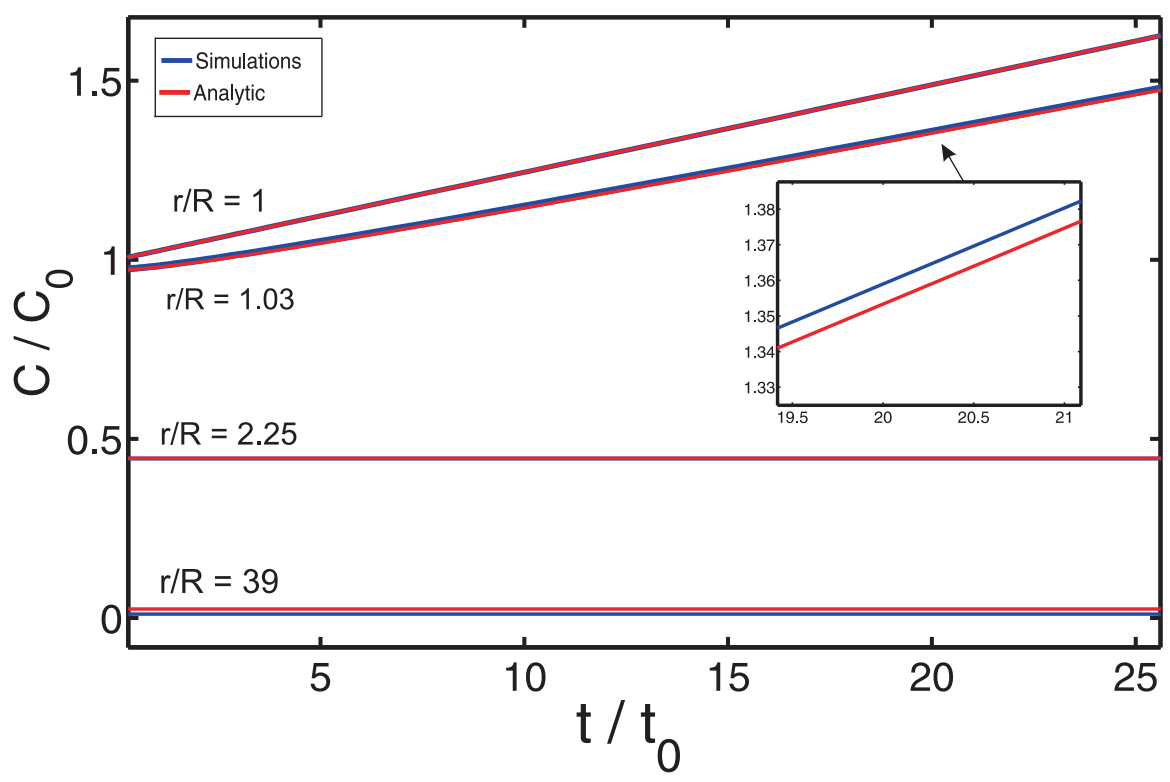

Figure 5.1: Evolution of the normalized gas concentration field in the liquid vs the normalized time, in the test case $(5.34),(5.35)$ from simulations (blue) and from analytical solution (red), for different $r / R=$ const; the normalization constants for concentration and time are $c_{0}=C$ and $t_{0}=t_{\text {diss }}$ respectively. 


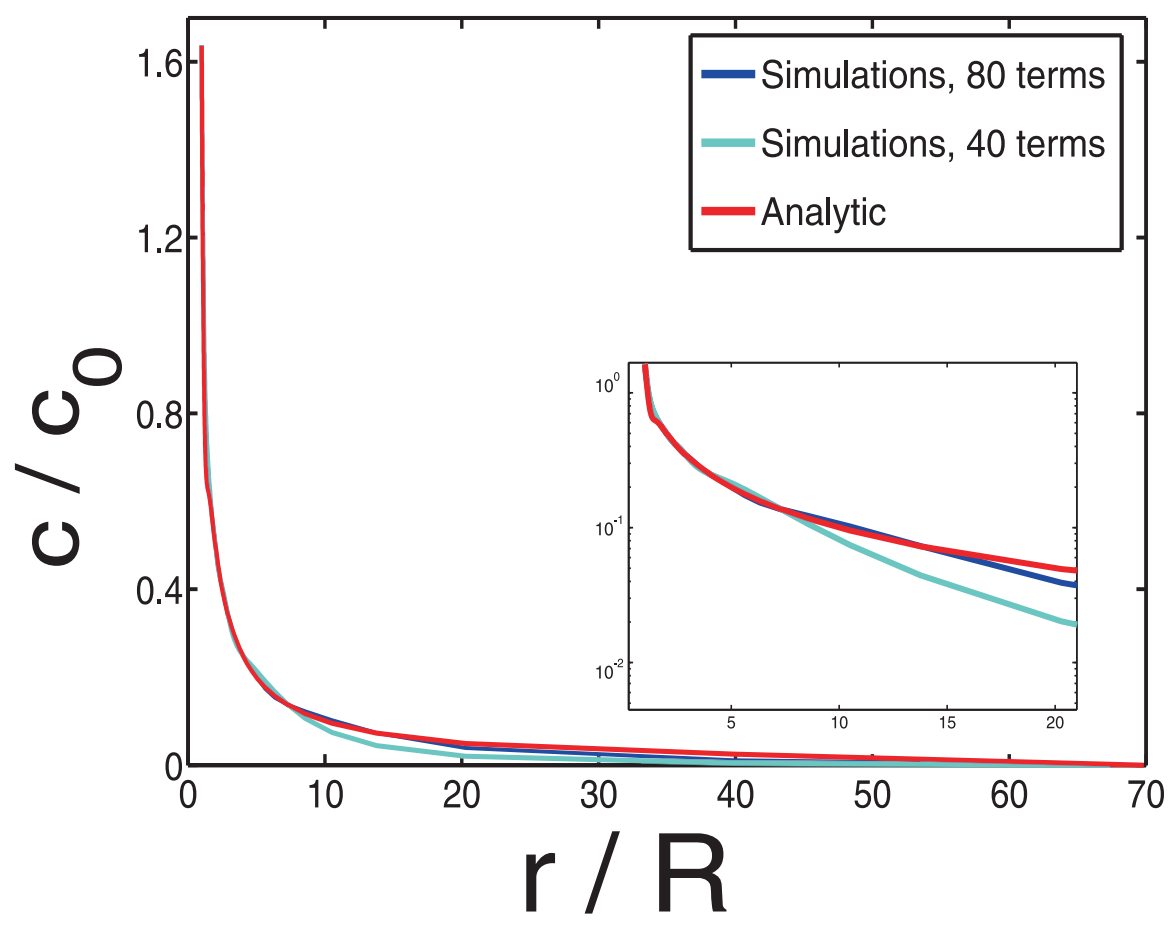

Figure 5.2: Normalized gas concentration field in the liquid at $t / t_{0}=26.1$ in the test case (5.34),(5.35). $r / R=1$ corresponds to the bubble wall; the normalization constants for concentration and time are $c_{0}=C$ and $t_{0}=t_{\text {diss }}$ respectively. Convergence is verified, as an increase from 40 terms (light blue) to 80 terms (blue) in (6.20) results into an almost perfect agreement between simulations and analytic results (red).

Upon substitution of (5.34),(5.35) inside (5.29), the following expression is found for $c(r, t)$ :

$$
\begin{aligned}
c(r, t)=\frac{R C}{r}+\frac{B R}{r}\{ & \operatorname{erfc}\left(\frac{r-R}{2 \sqrt{D t}}\right)\left[t+\frac{(r-R)^{2}}{2 D}\right] \\
& \left.-\frac{r-R}{\sqrt{D \pi}} \sqrt{t} \exp \left[-\frac{(r-R)^{2}}{4 D t}\right]\right\}
\end{aligned}
$$

We consider a $\mathrm{CO}_{2}$ bubble with fixed radius $R=3 \mu \mathrm{m}$. The values of the constants in (5.34) and (5.35) are chosen as $C=H p_{0}$ with $p_{0}=p_{\infty}+2 \sigma / R_{0}$ in which $p_{\infty}=1$ atm and $B=1 / \tau_{\text {diss }}$. Convergence was verified as the number of terms in (6.20) and (6.19) increased (see Fig. 5.2) and a satisfactory agreement was found between analytical and numerical solution (see Fig. 5.1). 


\subsection{Bubble nucleating next to a wall}

\subsubsection{Experimental setup}

A sketch of the experimental setup is shown in Fig. 5.3.

The system is composed of two stainless-steel tanks with volumes of 7 and 1.3 liters respectively. The larger one, the mixing tank, serves as a reservoir where a solution of water saturated with gas at pressure $p_{1}$ is prepared and stored. This mixture is then transferred to the smaller observation tank where the experiments of controlled bubble growth take place. A system of steel pipes and pneumatic valves connects the tanks to each other, to the water and gas sources as well as to the drainage system of the lab. At the beginning of the experiment, the observation tank is filled with fully gas-saturated liquid at pressure $p_{1}$. Then the pressure in the observation tank is dropped to the value $p_{2}<p_{1}$, therefore the liquid becomes supersaturated and bubbles start to grow at various possible nucleation sites. On the bottom of the tank a silicon wafer with artificial nucleation sites of radius $R_{p i t}=10 \mu \mathrm{m}$, has been placed, similar to the substrates used in the experiments of Refs. [5-8]. These substrates have shown to provide bubble nucleation at well controlled locations. The gas dissolved is $\mathrm{CO}_{2}$ and the liquid is water. This mixture is convenient due to the high solubility of $\mathrm{CO}_{2}$ in water $\left(\sim 1.6 \mathrm{~g}_{\mathrm{CO} 2}: 1 \mathrm{~kg}_{\mathrm{H} 2 \mathrm{O}}\right.$ at $\mathrm{T}=20^{\circ} \mathrm{C}$ and $p=1$ bar) compared to other gases.

The pressure $p_{1}$ in the mixing tank is controlled through a regulator placed on the $\mathrm{CO}_{2}$ line of the laboratory and it is measured with a pressure sensor read out by a multi-parameter transmitter communicating with the general control interface.

The pressure in the observation tank $p_{2}$ is measured and controlled by a pressure regulator and a flow controller. Since this type of control requires a certain controlled volume, an extra volume of $500 \mathrm{ml}$ is placed between the measurement vessel and the flow controller to enable a smooth regulation of the pressure.

\subsubsection{Comparison between experimental results and simulations}

When the pressure in the observation tank is lowered thus bringing the liquid to a supersaturation condition, the bubble nucleates attached to the wall, pinched to the edge of the pit. In the initial phase the bubble cannot be regarded as spherical, therefore the model hereby developed cannot give reliable predictions. However, after $\sim 100 \mathrm{~s}$, the bubble becomes almost spherical and it remains such until the moment it starts to deform just some seconds before detachment (see Fig. 5.4.f). We found that, with the exception of the initial stage, the present model is able to give a good prediction of the bubble growth, provided that a proper geometrical correction is introduced to 


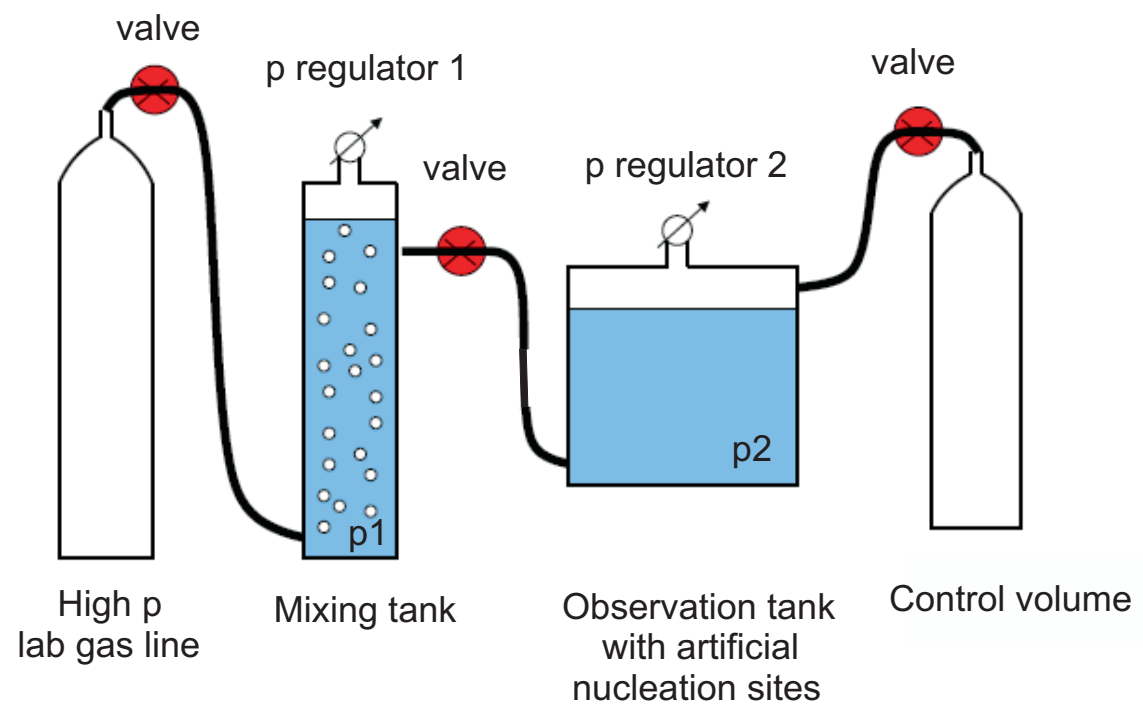

Figure 5.3: Experimental setup

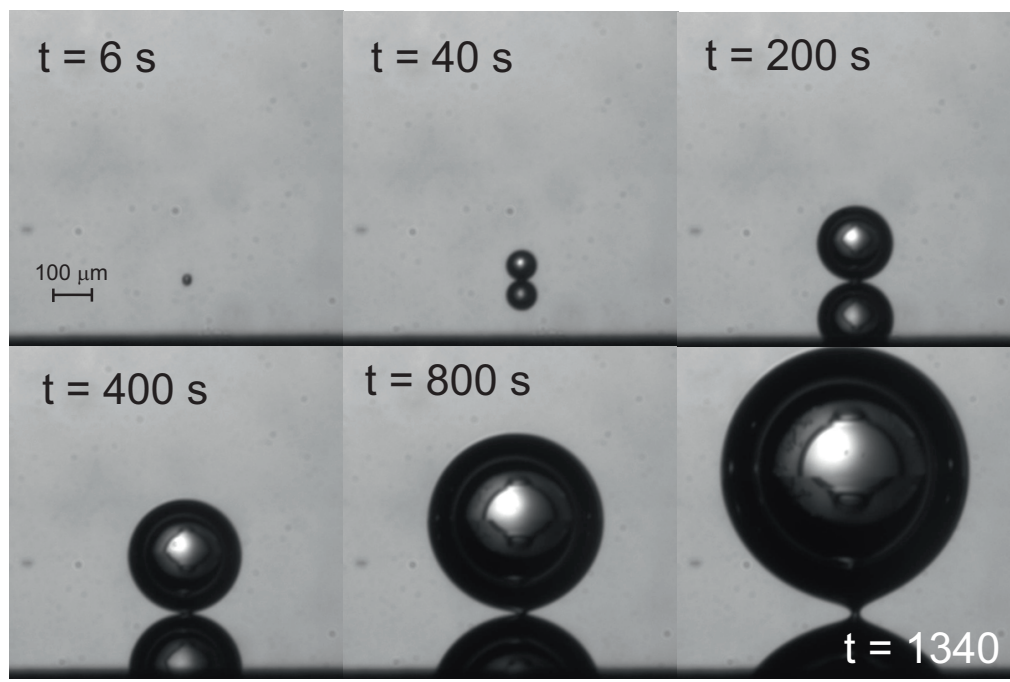

Figure 5.4: Snapshots of the nucleating bubble at different instants, from the moment that $p_{2}$ is dropped to 6 bar; $T_{\infty}=20^{\circ} \mathrm{C}, c_{\infty}=1.08 \times c_{s a t}$. 


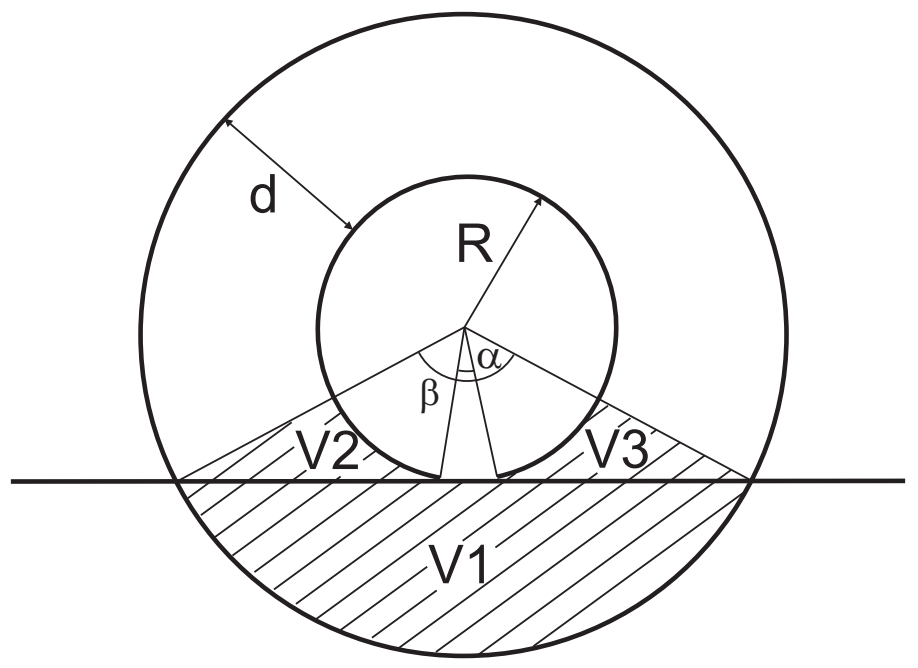

Figure 5.5: Sketch of the nucleated bubble and its boundary layer $d(t) . \alpha$ is the angle denoting the area excluded from the diffusive process because of the presence of the pit, $\beta$ is the angle denoting the excluded area because of the boundary layer disruption at the wall.

keep into account the presence of the wall.

We verified that the effect of the area excluded from the diffusive process due to the presence of the pit (i.e. the area subtending the angle $\alpha$ in Fig. 5.5) is not relevant in the considered time frame, as the pit has a small radius compared to the bubble, which can therefore be regarded as a perfect sphere tangent to the nucleation plane. However, the effect of the disruption of the boundary layer due to the presence of the wall turned out to be of primary importance in explaining the dynamics of the bubble. We introduced a geometrical correction in order to exclude the area subtending the angle $\beta$ in Fig. 5.5, thus transforming the mass conservation law (6.15) into

$$
\dot{m}_{G}=\left.\left(4 \pi R^{2}-A_{e x c l}\right) D \frac{\partial c}{\partial r}\right|_{R},
$$

where $A_{\text {excl }}=2 \pi R^{2}\left(1-\cos \frac{\beta}{2}\right)$ is the excluded area due to the boundary layer disruption. The angle $\beta(t)$ has been calculated with basic geometric considerations from a linear estimate of the thickness of the boundary layer $d(\mathrm{t})$ :

$$
d(t)=\frac{c_{\infty}-\left.c\right|_{R}}{\left.\frac{\partial c}{\partial r}\right|_{R}},
$$


in which $\left.\frac{\partial c}{\partial r}\right|_{R}$ is the gradient of the concentration at the bubble interface for a spherical bubble in an infinite medium. This correction reflects the fact that introducing a wall forces the gradient of the concentration to vanish. If the wall is introduced far from the bubble, where the concentration field is almost flat as compared to the radial coordinate $r$, its effect will be negligible. On the contrary, if it is introduced next to the bubble wall, where $\frac{\partial c}{\partial r}$ is maximal, its effect will be important. Strictly speaking, only the mass of gas contained inside the volume $V_{1}$ in Fig. 5.5 should be excluded from the mass flux entering the bubble, while the contribution of $V_{2}$ and $V_{3}$ should be considered. However, we assumed for simplicity that the effect of excluding $V_{2}$ and $V_{3}$ was negligible.

In Fig. 5.6, we track the time evolution of a $\mathrm{CO}_{2}$ bubble created by lowering the pressure in the observation tank from $p_{1}=7$ bar to $p_{2}=6$ bar, corresponding to a supersaturation degree of 1.08 , and we compare it with numerical results. In the simulations we took as initial radius $R_{0}=130 \mu \mathrm{m}$, and as initial concentration field

$$
c_{0}(r, t)=c_{\infty}-\left(c_{s}-c_{\infty}\right) \exp \left(-\frac{r-R_{0}}{d_{0}}\right) .
$$

Here $d_{0}$ is the thickness of the boundary layer when the simulation starts, estimated as $d_{0}=\sqrt{\pi D t_{0}}$, with $t_{0}$ the instant when the bubble has dimension $R_{0}$ in the experiment. This estimate comes from the solution of the standard diffusion equation [9]. With the geometrical correction, we found a satisfactory agreement between experimental and numerical results, that could not be retrieved without. We also managed to reproduce the non-square-root-like behavior emerging from the experiments and differing from the one expected after the short initial surface tension and inertia dominated phases, for a bubble growing by pure diffusion inside an infinite medium [10] (see Fig. 5.7). 


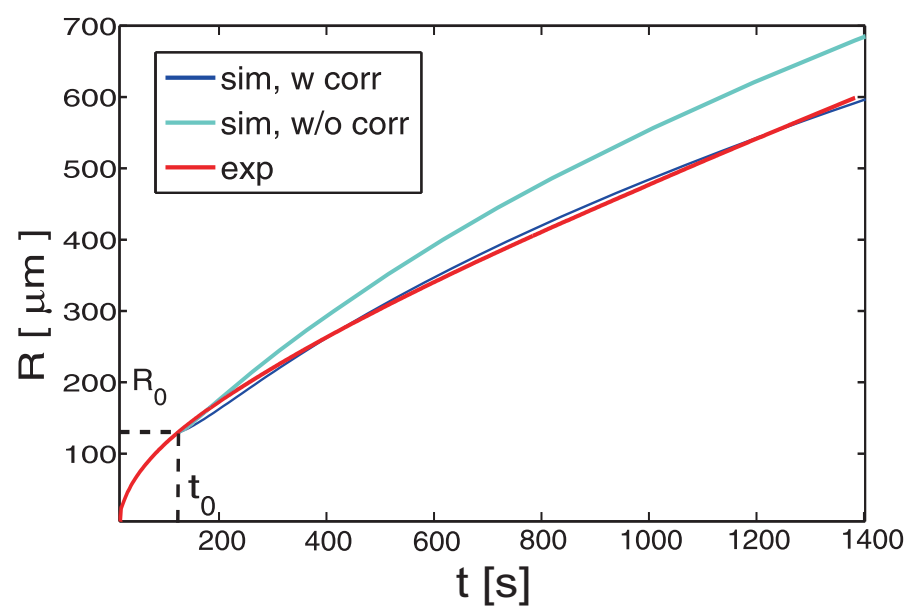

Figure 5.6: $\mathrm{R}(\mathrm{t})$ curves from experiments (red) and simulations, both with (blue) and without (light blue) geometric correction. Parameters: $T_{\infty}=20^{\circ} \mathrm{C}, c_{\infty}=1.08 \times c_{s a t}$, $t_{0}=120 \mathrm{~s}, R_{0}=130 \mu \mathrm{m}$.

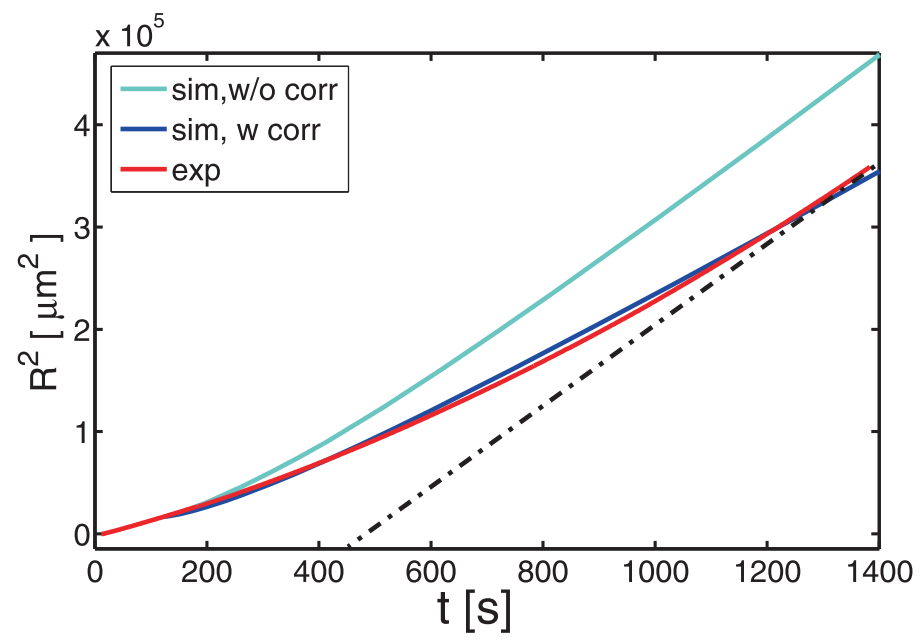

Figure 5.7: $\mathrm{R}^{2}(\mathrm{t})$ curves from experiments (red) and simulations, both with (blue) and without (light blue) geometric correction. Parameters: $T_{\infty}=20^{\circ} \mathrm{C}, c_{\infty}=1.08 \times c_{\text {sat }}$, $t_{0}=120 \mathrm{~s}, R_{0}=130 \mu \mathrm{m}$. The black line represents the slope of the square root growth of a bubble growing purely by mass diffusion inside an infinite medium. 


\subsubsection{Conclusions}

In view of a future extension of the PDE model presented in Chap. 2, in the present chapter we developed a model for a cavitation bubble growing by mass diffusion, including both the gas concentration field inside the liquid and the temperature field inside the bubble. We validated it by comparing it both with the analytical solution in a test case and with the experimental results regarding the time evolution of a bubble growing purely by mass diffusion, attached to a pit. We introduced a geometric correction to keep into account the disruption of the boundary layer due to the presence of the wall. Though the very first phase of the bubble growth cannot be reproduced by the model, due to the asymmetry and lack of sphericity of the observed phenomenon, this correction allows to predict the evolution of the bubble with a high accuracy, as the spherical symmetry of the bubble is regained. In particular, it allows to reproduce the non-square-root-like behavior observed in the experiments. This result is particularly interesting because it differs from classical square-root behavior of a bubble growing purely by mass diffusion inside an infinite medium [10]. 


\section{References}

[1] A. Prosperetti, L. A. Crum, and K. W. Commander, "Nonlinear bubble dynamics", J. Acoust. Soc. Am. 83, 502-514 (1988).

[2] G. K. Batchelor, An Introduction to Fluid Dynamics (Cambridge University Press, Cambridge) (1970).

[3] L. Stricker, A. Prosperetti, and D. Lohse, "Validation of an approximate model for the thermal behavior in acoustically driven bubbles", J. Acoust. Soc. Am. 130, 3243-3251 (2011).

[4] A. Prosperetti, Advanced mathematics for applications (Cambridge Univ. Press, Cambridge) (2011).

[5] N. Bremond, M. Arora, C. D. Ohl, and D. Lohse, "Controlled multibubble surface cavitation”, Phys. Rev. Lett. 96, 224501 (2006).

[6] N. Bremond, M. Arora, S. M. Dammer, and D. Lohse, "Interaction of cavitation bubbles on a wall”, Phys. Fluids 18, 121505 (2006).

[7] B. Borkent, S. Gekle, A. Prosperetti, and D. Lohse, "Nucleation threshold and deactivation mechanisms of nanoscopic cavitation nuclei”, Phys. Fluids 102003 (2009).

[8] D. Fernández Rivas, L. Stricker, A. Zijlstra, H. Gardeniers, D. Lohse, and A. Prosperetti, "Ultrasound artificially nucleated bubbles and their sonochemical radical production”, Ultrason. Sonochem. 20, 510-524 (2013).

[9] A. Bejan, Heat transfer (John Wiley and Sons, Inc., New York) (1993).

[10] M. S. Plesset and S. A. Zwick, "The growth of vapor bubbles in superheated liquids”, J. Appl. Phys. 25, 493-499 (1954). 


\section{6 \\ The role of gas in ultrasonically driven vapor bubble growth ${ }^{*} \dagger$}

In the present work we study both experimentally and theoretically the dynamics of an ultrasound driven vapor bubble of perfluoropentane (PFP) inside a droplet of the same liquid, immersed in a water medium superheated respect to the PFP boiling point. We determine the temporal evolution of the bubble radius with ultra-high speed imaging at 20 million frames per second. In addition we model the vapor-gas bubble dynamics, based on a Rayleigh-Plesset-type equation, including thermal and gas diffusion inside the liquid. We compare the numerical results with the experimental data and find good agreement. We underline the fundamental role of gas diffusion in order to prevent total recondensation of the bubble at collapse.

\subsection{Introduction}

Current chemotherapeutical cancer treatment has severe side effects caused by the adverse outcome of the drugs on healthy tissue. Therefore it is desirable to create

*Submitted to Phys. Med. Biol. as: [Oleksandr Shpak*, Laura Stricker*, Michel Versluis and Detlef Lohse, The role of gas in ultrasonically driven vapor bubble growth. *These authors contributed equally to the present work].

$\dagger$ The experimental data present in this chapter are entirely due to Oleksandr Shpak. 
drug delivery agents, which will release the drug locallly at the tumor site. Recent studies have shown that liquid emulsion phase-change droplets composed of perfluorocarbons (PFC) such as perfluoropentane (PFP) have the potential to be a highly efficient system for local drug delivery $[1,2]$. PFP liquid has a boiling temperature of $29^{\circ} \mathrm{C}$. However, when stabilized the submicron- and micron-sized PFP droplets do not spontaneously vaporize when injected in vivo at $37^{\circ} \mathrm{C}$ temperature $[3,4]$. A phase transition can be triggered by application of an acoustic pusle of sufficiently high pressure $[3,5]$. An explosive evaporation takes place and the drug is released from the liquid core [6]. The advantage of the so-called acoustic droplet vaporization (ADV) technique are its non-invasive and inexpensive character. Moreover, the resulting bubbles become acoustically active ultrasound contrast agents, which are detectable for at least one microsecond after nucleation [7]. Although the success of this approach for localized drug delivery has been demonstrated [1], the physical mechanisms underlying the ADV process have not been well described until now [8].

In this article we use ultra-high speed optical imaging to study experimentally the ultrasound driven vapor bubble growth in the superheated liquid at nanoseconds timescale. We capture the vaporization dynamics of a single emulsion system: PFP droplets of a radius of $5 \mu \mathrm{m}$ immersed in water. This system is simpler to model and to track experimentally with respect to multiple phase emulsions used for localized drug delivery $[6,9]$. Some theoretical work has already been done on the modelling of acoustic droplet vaporization at microseconds time scale, in the frame of gas embolotherapy, a promising cancer treatment involving tumor occlusion with gas microbubbles selectively formed from the liquid droplets [10]. Here we focus on the nanoseconds time scale in order to capture the complex interaction of the ultrasound with the vapor bubble. We develop a PDE model based on a Rayleigh-Plesset-type equation, including thermal and gas diffusion inside the PFP liquid. We compare the experimental data with the numerical results, finding a good agreement. Such a model can therefore provide a valuable instrument for practical purposes, where one wants to control the size of resulting bubbles as a function of time, for example when they are used as ultrasound contrast agents [7]. We underline the fundamental role of gas diffusion, in order to prevent total recondensation of the bubble at collapse, provided that the periodic ultrasound driving is present. Recondensation of a vapor bubble was thought as a possibility to induce reiterated phase transition of the same droplet. For applications such as sonoporation and tumor imaging this possibility may be of a crucial importance in order to avoid repeated injections of ADV agents.

Mathematical models for spherically symmetric vapor-gas bubbles have already been formulated in Refs. [11-17], and their small oscillations have been analytically investigated in detail in Refs. $[12,17]$. In the present work we will show that 
our approach has two great advantages with respect to the earlier models. First, it is simpler, as it does not include diffusion of the gas inside the vapor, though still able to capture the relevant experimental features. And secondly, it does not present fitting parameters such as the accommodation coefficient for the description of the evaporative process $[11,12]$.

\subsection{Materials and Experimental Methods}

The PFP droplet emulsion was prepared with bovine serum albumin using the same method adopted in Refs.[18, 19]. The resulting highly diluted suspension of droplets was injected into an OptiCell (Thermo Fisher Scientific, Waltham, MA, USA) in order to observe the droplets individually. The OptiCell was submerged in a 1 liter temperature-controlled water tank. A schematic of the experimental setup is shown in Figure 6.1. Microdroplets were phase-transitioned with a focused 1.5-inch diameter single-element transducer (A381S; Olympus Panametrics, Antwerpen, Belgium, 3.5 MHz center frequency, NA 1.0).

The acoustic driving pulse was generated by an arbitrary waveform generator (Tabor 8026; Tabor Electronics, Tel Hanan, Israel) amplified by a RF-amplifier (ENI 350L, Electronic Navigation Industries, Inc., Rochester, NY, USA). Droplet samples were vaporized with a single ultrasound pulse, consisting of a burst of 6 cycles and a driving pressure $P_{a}$ of $4.5 \mathrm{MPa}$. The pressure value was calibrated in the same setup at the position of the droplets using a $0.2 \mathrm{~mm}$ PVDF probe hydrophone (DC27/000658, Precision Acoustics Ltd., Dorchester, UK). An Olympus microscope (Olympus; Zoeterwoude, The Netherlands) with a 40x water immersed objective (NA $=0.7$ ) was focused on an OptiCell providing a spatial resolution of $0.269 \mu \mathrm{m}$ per pixel. The radial response of the insonified droplets was recorded with an ultra-fast recording camera, the Brandaris 128 (Chin et al. 2003). The camera was set to record 6 movies of 128 frames in a single run at a frame rate up to 20 million frames per second (fps), thus providing an interframe time as low as 50 nanoseconds. The first recording was always done without ultrasound to estimate the noise level of the system. The region of interest was illuminated with a $65 \mathrm{~mJ}$ Xenon flash pulse $(30 \mu \mathrm{s}$ duration) through the light guide (SCHOTT AG, Mainz, Germany) from below. From the movies, the radii $R(t)$ of the resulting droplets as a function of time were measured using custom-designed image analysis software based on a minimum cost function. The droplet radius was calculated as the mean distance from the center of mass to the edge of the droplet in a radial coordinate system averaged over the full $2 \pi$ angle. The vapor bubble dynamics model used here (see Sect. 3) assumes spherical symmetry of the problem. However, due to the homogeneous bubble nucleation nature of ADV, 


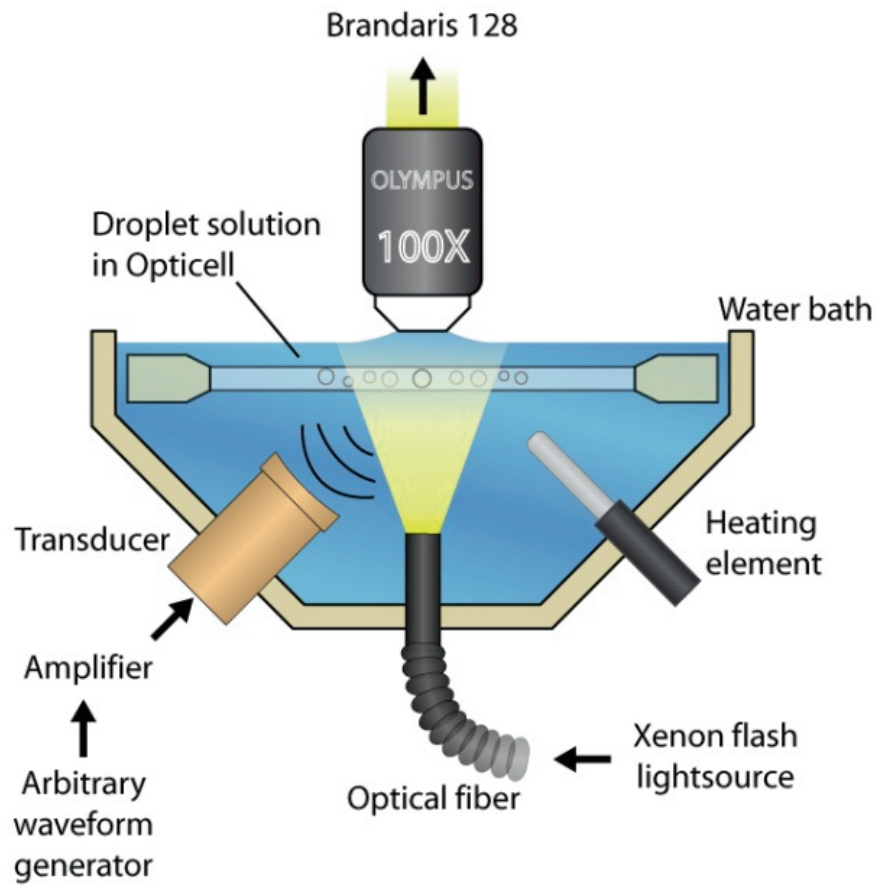

Figure 6.1: Schematic view of the experimental setup. An OptiCell polystyrene container with the microdroplet suspension was positioned inside a water tank kept to a constant temperature $T_{\infty}$, superheated with respect to the PFP boiling point. A focused transducer sent the US-pulses from a side with an inclination of $45^{\circ}$ with respect to the plane of the Opticell. The samples were imaged from the top with an upright microscope coupled to the Brandaris 128 high-speed camera and irradiated from below with a Xenon flash light.

the vapor nucleus appears not necessarily in the center of the droplet. In order to be able to compare the numerical results with experimental data, we chose a case in which the nucleation occured well centered within the droplet.

\subsection{Model}

In the model we consider a spherical bubble of PFP inside an infinite medium of liquid PFP, neglecting the surrounding water. We consider both the vapor and the gas inside the bubble and we model their dynamics independently, i.e. we neglect the diffusion of gas inside the vapor in the bubble. Both the gas and the vapor inside the 


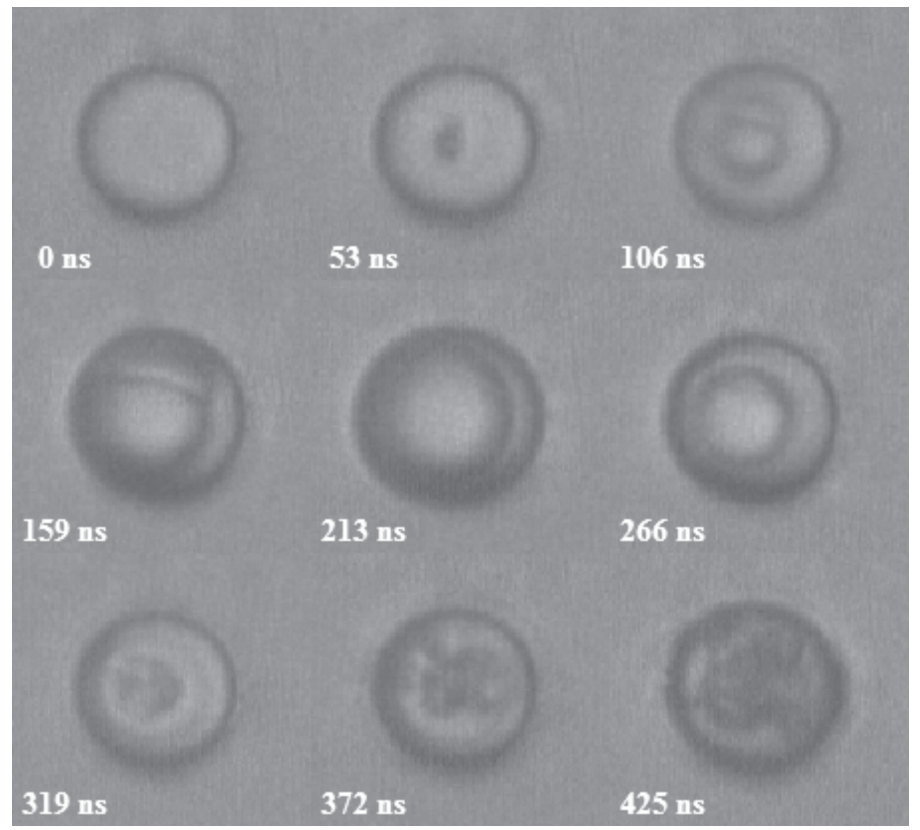

Figure 6.2: A set of consecutive images showing acoustic droplet vaporization at $15^{\circ} \mathrm{C}$ superheat, taken at a frame rate of $18.81 \mathrm{Mfps}$. Driving parameters: frequency $f=3.5 \mathrm{MHz}$, pressure $\mathrm{Pa}=4.5 \mathrm{MPa}$. The initial radius of the droplet is $R_{0}=5.38 \mu \mathrm{m}$. The vapor bubble inside the droplet undergoes vaporization/(re-)condensation cycles with the frequency of the applied ultrasound.

bubble are treated as perfect gases in saturated conditions. For a complete description of the vapor dynamics and the evolution of the thermal field, we refer the reader to Refs.[20, 21] and Ref. [22] respectively. We assume a spatially uniform pressure inside the bubble, low Mach number regimes, spherical symmetry and shape stability. The gas considered in the simulations is air with standard composition.

The radial dynamics of the bubble is described by a Rayleigh-Plesset-type equation with the formulation used in Ref. [22]:

$$
\left(1-\frac{\dot{R}}{c_{L}}\right) R \ddot{R}+\frac{3}{2}\left(1-\frac{\dot{R}}{3 c_{L}}\right) \dot{R}^{2}=\frac{1}{\rho_{L}}\left(1+\frac{\dot{R}}{c_{L}}+\frac{R}{c_{L}} \frac{d}{d t}\right)\left[p_{B}-p_{A}\right],
$$

in which $R$ is the bubble radius, $\rho_{L}$ is the density of the liquid, $c_{L}$ is the speed of sound and the dot is used to indicate time derivatives. The ambient pressure in the liquid, $p_{A}$, is calculated as

$$
p_{A}=p_{\infty}-P_{a} \cos \omega t
$$


with $P_{a}$ the acoustic driving pressure, $f=\omega / 2 \pi$ the driving frequency and $p_{\infty}$ the static pressure. Such a phase of the driving pressure has been taken because the bubble nucleation is most likely to occur at the negative pressure peak, when the stretching of the liquid is maximal [19]. The pressure at the bubble surface on the liquid side, $p_{B}$, is derived from the normal stress balance at the bubble wall

$$
p=p_{B}+4 \mu_{L} \frac{\dot{R}}{R}+\frac{2 \sigma}{R},
$$

where $\mu_{L}$ is the dynamic viscosity of the liquid and $\sigma$ is the surface tension. The total pressure inside the bubble, $p$, can be expressed as the sum of the partial pressures of gas $p_{G}$ and vapor $p_{V}$ :

$$
p=p_{V}+p_{G}
$$

The Clausius-Clapeyron relation holds [21]:

$$
\frac{d p_{V}}{d T_{S}}=\frac{L \rho_{V}}{T_{S}},
$$

where $L$ is the latent heat and $\rho_{V}$ the density of the vapor. An explicit expression for $p_{V}$ can be found by integrating Eq. (6.5) with the further use of the perfect gas law of the vapor

$$
p_{V}=P_{0} \exp \left(-\frac{L}{R_{V} T_{S}}\right) .
$$

Here $P_{0}=p_{V, 0} \exp \left(\mathrm{L}_{\mathrm{RT}}\right)$ is a constant and $R_{V}$ is the universal gas constant divided by the molecular mass of the vapor. The pressure of the gas $p_{G}$ is related to the concentration of the gas in the liquid at the bubble wall, $\left.\mathrm{c}\right|_{R}$, by Henry's law:

$$
p_{G}=\left.k_{H} c\right|_{R}
$$

where $\mathrm{k}_{H}$ is the Henry's constant, derived from air solubility data inside PFP.

We assume a uniform temperature field inside the bubble. This is a common assumption for vapor bubbles and its validity has been verified in Ref. [20]. In order to describe the evolution of the temperature field in the liquid $T(r, t)$ we use the standard convection-diffusion equation [22]:

$$
\rho_{L} c_{p, L}\left(\frac{\partial T}{\partial t}+\frac{R^{2} \dot{R}}{r^{2}} \frac{\partial T}{\partial r}\right)=k_{L} \nabla^{2} T
$$

where $r$ is the radial coordinate measured from the bubble center, while $c_{p, L}$ and $k_{L}$ are the specific heat and thermal conductivity of the liquid. Far from the bubble, the 
liquid temperature is assumed to remain undisturbed at the initial value $T_{\infty}$ and the following boundary condition is adopted:

$$
\frac{\partial T}{\partial r}(\infty, t)=0 .
$$

At the bubble wall, the temperature is continuous. Therefore:

$$
T(R(t), t)=T_{S}(t),
$$

i.e. $T_{S}$ - the temperature at the surface - equals the temperature inside the bubble. The energy balance at the bubble wall can be written as [20]:

$$
L \frac{d}{d t}\left(\rho_{V} V\right)+\rho_{V} V c_{s} \frac{d T_{S}}{d t}=\left.4 \pi R^{2} k \frac{\partial T}{\partial r}\right|_{R},
$$

with $c_{S}=c_{P, V}-L / T_{S}$ the specific heat of the saturated vapor, and $V$ the volume of the bubble. The first term in Eq. (6.11) is the heat employed to evaporate the liquid, the second is the change of vapor enthalpy in time due to temperature changes and the third one is the heat transferred by conduction from the liquid to the bubble. The change of gas enthalpy in time due to temperature changes has been neglected as we assumed $\rho_{G}<<\rho_{V}$. This assumption turned out to be justified because we found $\rho_{G} / \rho_{V} \sim 10^{-2}$, as will be shown later. We also neglected the heat flux by diffusion inside the bubble because of the assumption of uniform temperature. By making use of vapor mass conservation, the Clausius Clapeyron eq. and the perfect gas law for the vapor, Eq. (6.11) can be rewritten as

$$
\left(c_{P}+\frac{L^{2}}{R T_{S}^{2}}-\frac{2 L}{T_{S}}\right) \frac{d T_{S}}{d t}=\frac{3}{\rho_{V} R}\left(\left.k \frac{\partial T}{\partial r}\right|_{R}-\rho_{V} L \dot{R}\right)
$$

from which an explicit expression for $\frac{d T_{S}}{d t}$ can be extracted. For a detailed derivation of Eq. (6.12) we refer the reader to Ref. [21].

Similarly to the temperature, the concentration of the gas in the liquid $c(r, t)$ is described by the standard convection-diffusion equation:

$$
\frac{\partial c}{\partial t}+\frac{R^{2} \dot{R}}{r^{2}} \frac{\partial c}{\partial r}=D_{G} \nabla^{2} c
$$

Here $\mathrm{D}_{G}$ is the mass diffusivity of the gas inside the liquid, calculated by means of the Stokes-Einstein formula [23]: $D_{G}=k_{B} T /\left(6 \pi \mu_{L} r_{G}\right)$, where $k_{B}$ is the Boltzmann's constant and $r_{G}$ is the gas molecular radius calculated as half of the collisional diameter, following Ref. [24]. Far from the bubble, the gas concentration is assumed to 
remain undisturbed at the initial value $c_{\infty}=0.68 \mathrm{~kg} / \mathrm{m}^{3}$, equal to the solubility of air inside PFP, and obviously, as a further boundary condition:

$$
\frac{\partial c}{\partial r}(\infty, t)=0
$$

Mass conservation of the gas implies:

$$
\frac{d}{d t}\left(\rho_{G} V\right)=\left.4 \pi R^{2} D_{G} \frac{\partial c}{\partial r}\right|_{R},
$$

where $\rho_{G}$ is the gas density inside the bubble, related to the gas pressure by perfect gas law

$$
\frac{p_{G}}{\rho_{G}}=R_{G} T_{S}
$$

with $R_{G}$ the universal gas constant divided by the molecular weight of the gas. All the properties of PFP were taken from Refs.[25, 26].

For the solution of both the energy equation Eq. (6.8) and the gas diffusion equation Eq. (6.13) we used the method presented in Ref. [22], i.e. a pseudo-spectral collocation method. The reader is referred to that reference for details. We adopted the coordinate transformation

$$
\frac{1}{\xi}=1+\frac{r / R(t)-1}{l},
$$

in order to map the semi-infinite range $R(t) \leq r<\infty$ into the finite range $1 \geq \xi \geq 0$. In (6.17), the constant $l$ is related to the thermal diffusion length in the liquid, as thermal diffusion is the dominating process in the observed phenomenon and it has been taken as

$$
l=\ell \frac{\sqrt{D_{L} / \omega}}{R_{0}},
$$

where $D_{L}=\lambda_{L} / c_{p, L} \rho_{L}$ is the liquid thermal diffusivity and $\ell$ is a constant number. We adopted a value of $\ell=20$, in agreement with results of Ref. [27]. We reformulated the energy equation and the gas diffusion equation by substituting the new variable $\xi$ inside the Eqs. (6.8) and (6.13), and we expanded the liquid temperature and the gas concentration fields in truncated Chebyshev series:

$$
\begin{gathered}
\frac{T(t, \xi)}{T_{\infty}} \approx \sum_{k=0}^{N} a_{k}(t) T_{2 k}(\xi), \\
\frac{c(t, \xi)}{c_{\infty}} \approx \sum_{k=0}^{N} b_{k}(t) T_{2 k}(\xi),
\end{gathered}
$$


in which $T_{2 k}(\xi)$ are the even Chebyshev polynomials. The coupled equations for the various $a_{k}(t)$ and $b_{k}(t)$ arising upon substitution of Eqs.(6.19) and (6.20) into Eqs. (6.8) and (6.13) were solved by collocation. Convergence was reached by using 45 terms in the Chebyshev expansions with 45 Gauss-Lobatto collocation points. In the limiting cases of the absence of gas diffusion, we have verified that the code developed for this work gave the same results as those used in Refs. [20] and in Chap. 5 respectively, which had been both validated against analytical solutions. In the case of pure gas diffusion and no driving an additional validation was performed against experimental data in Chap. 5.

\subsection{Results}

In experiments we measured the external radius of the droplet as a function of time. The statistical error of the measurement of the droplet radii was $150 \mathrm{~nm}$. Assuming incompressibility for liquid PFP, we extracted the radius $R(t)$ of the bubble, using mass conservation: $\rho_{L}\left(V_{d}(t)-V(t)\right)+\rho_{V} V(t)=\rho_{L} V_{d, 0}$, where $V_{d}(t)$ and $V(t)$ are the volume of the droplet and the volume of the bubble, respectively, $V_{d, 0}$ is the initial volume of the droplet, before the bubble nucleates, and $\rho_{L}$ is the density of liquid PFP. Therefore

$$
R(t)=\sqrt[3]{\frac{\rho_{L}}{\rho_{L}-\rho_{V}}\left(R_{d}^{3}(t)-R_{d, 0}^{3}\right)}
$$

with $R_{d, 0}$ the initial radius of the droplet before the bubble nucleates and $R_{d}(t)$ the measured radius of the droplet. In Fig. 6.3 we compare the time evolution of the bubble radius extracted from the experimental data with simulations. We chose a case with one well isolated bubble remaining almost spherical and far from the droplet wall (see Fig. 6.2).

A 4.5 MPa pressure driving pulse with a frequency of $3.5 \mathrm{MHz}$ for the duration of six cycles was applied. The measurements of the driving pressure amplitude performed with the hydrophone were accurate within a relative error of up to $200 \mathrm{kPa}$, based on the standard deviation of the acoustic pressure amplitudes required to activate droplets of the same size [28]. We took as initial radius $R_{0}$ for the simulations the lowest value inferred from experiments, which is $1 \mu \mathrm{m}$. The water surrounding the droplet was kept at the constant temperature $T_{\infty}=44^{\circ} \mathrm{C}$, i.e. with a superheat of $15^{\circ} \mathrm{C}$ with respect to the boiling point of PFP $T_{b}=29^{\circ} \mathrm{C}$. The error of the temperature measurements was estimated to be $1^{\circ} \mathrm{C}$, due to the circulation of water above the heating element. As the nucleation of the vapor bubble is an abrupt phenomenon, ideally we should consider as initial condition a constant temperature field equal to the water temperature, in the whole liquid PFP and the boiling temperature of PFP at 
the bubble wall. For numerical reasons, we smeared out this step condition and we took instead an initial temperature field $T_{0}(r)$ as

$$
T_{0}(r)=T_{\infty}-\left(T_{\infty}-T_{b}\right) \exp \left(-\frac{r-R_{0}}{\delta-R_{0}}\right),
$$

where $\left(\delta-R_{0}\right)$ describes the thickness of the thermal boundary layer. We tried different values for $\delta, R_{0}<\delta \leq 2 R_{0}$ and we found that, as long as the initial boundary layer is restrained to one radius distance from the bubble wall, its effective thickness does not influence significantly the final result.

Similarly, for the gas concentration inside the liquid, we assumed an initial distribution $c_{0}(r)$, defined as

$$
c_{0}(r)=c_{\infty}-\left(c_{\infty}-c_{b}\right) \exp \left(-\frac{r-R_{0}}{\delta^{\prime}-R_{0}}\right)
$$

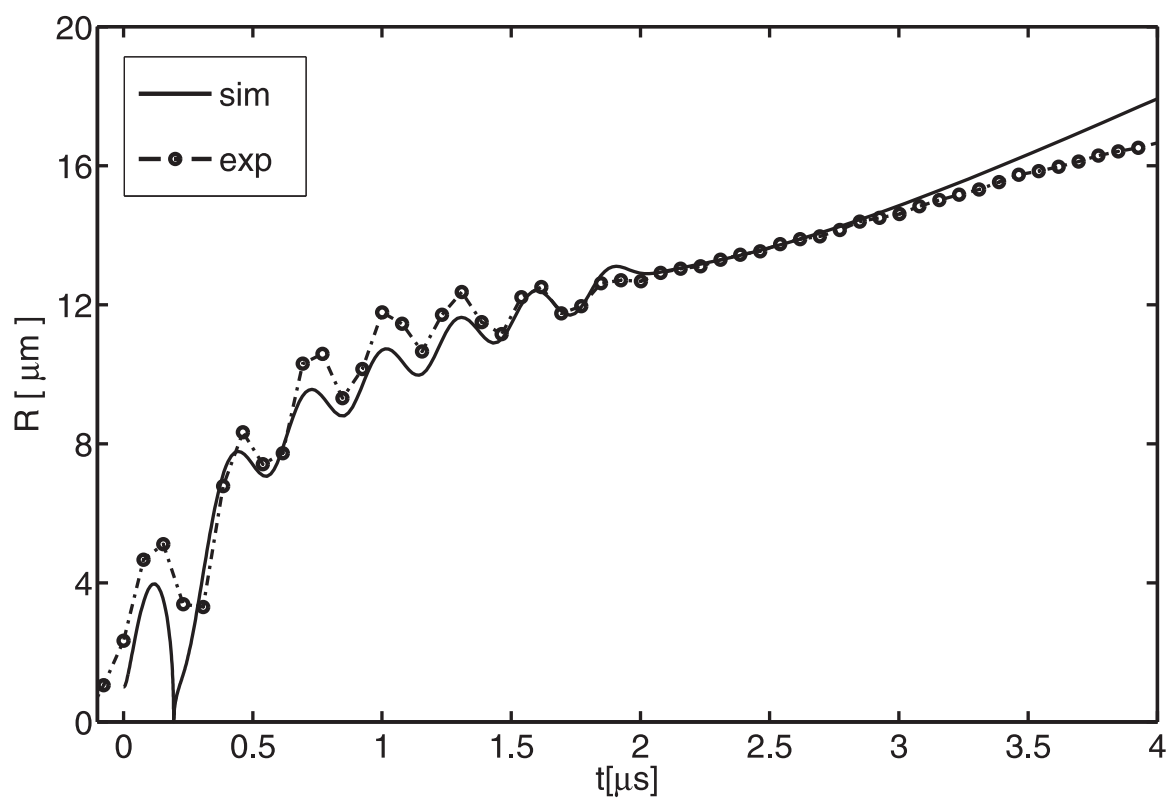

Figure 6.3: Radius-time curve $R(t)$ of a PFP bubble from experiments (dash-dot) and simulations (solid) at $15^{\circ} \mathrm{C}$ superheating. Driving parameters: frequency $f=$ $3.5 \mathrm{MHz}$, pressure $P a=4.5 \mathrm{MPa}$. After $2 \mu$ s the driving was turned off. The initial radius for simulations $R_{0}=1 \mu \mathrm{m}$ has been taken from the first value detected in experiments during the non-instantaneous nucleation phase. 


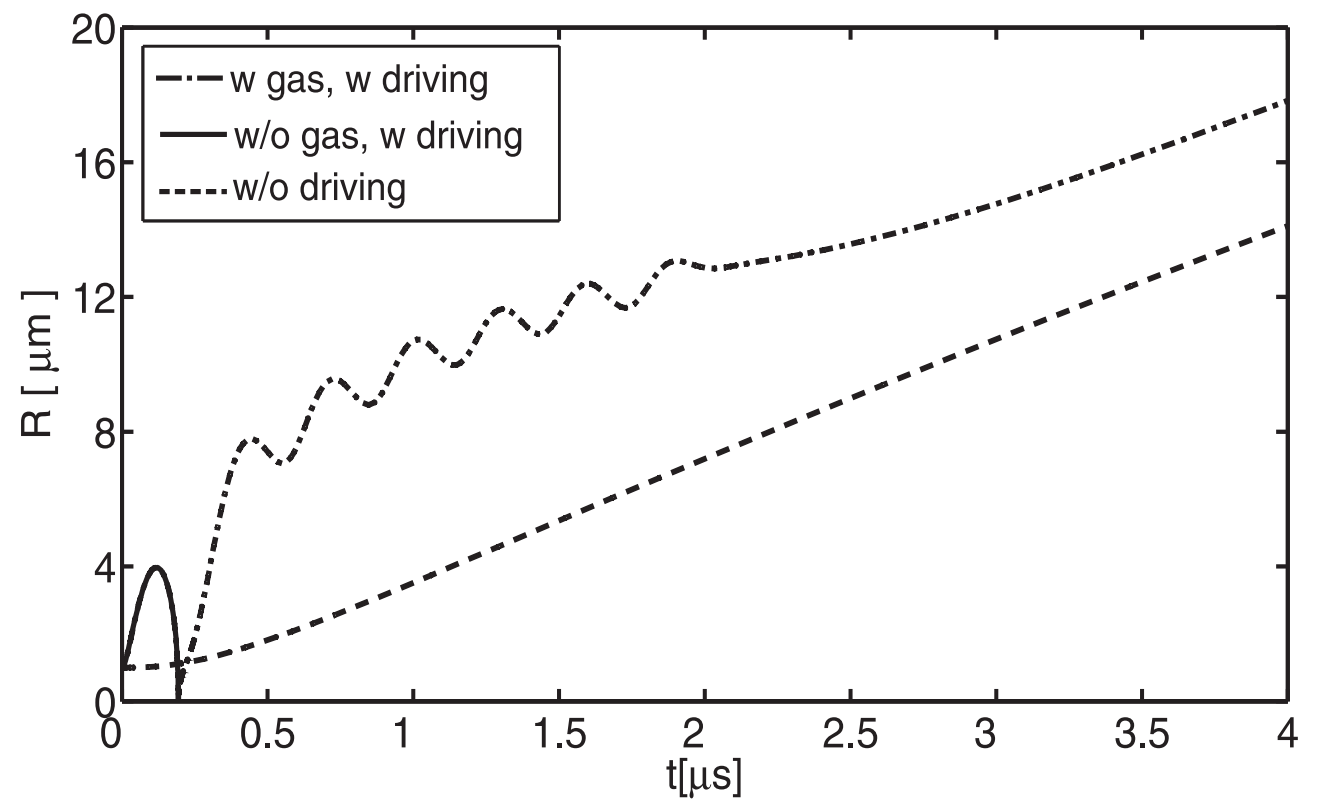

Figure 6.4: Radius time curve $R(t)$ from simulations for a $1 \mu \mathrm{m}$ bubble in the same conditions of Fig.6.3 without (dash) and with driving, both with (dash-dot) and without (solid) gas diffusion. When the bubble is forced and without gas diffusion the bubble undergoes complete condensation after the first collapse. When the bubble is unforced, gas diffusion has no effect on the bubble dynamics.

where $\left(\delta^{\prime}-R_{0}\right)$ describes the thickness of the mass diffusive boundary layer. Here $c_{\infty}=0.6846 \mathrm{~kg} / \mathrm{m}^{3}$ is the concentration of air inside PFP, given by the solubility of air inside PFP at $1 \mathrm{~atm}$, and $c_{b}$ is the initial concentration of gas at the bubble wall, set by Henry's law. We assumed the initial amount of gas trapped inside the bubble to be zero, therefore $c_{b}=0 \mathrm{~kg} / \mathrm{m}^{3}$. The same considerations reported for the initial temperature field apply also to the initial gas concentration field. At initial stages the discrepancies between experimental data and simulations may be attributed to the shape instabilities of the vapor bubble after the first rebounce. At later stages the vapor bubble approaches the boundary of a PFP droplet and the assumption of infinite PFP liquid is no longer valid.

The role of gas diffusion inside the bubble turns out to be of primary importance, provided acoustic forcing is present. Although the rate of gas to vapor mass $m_{G} / m_{V}$ is of order $10^{-2}$, gas diffusion is necessary to avoid complete condensation of the bubble after the first collapse of the vapor bubble. Fig. 6.4 shows that, without in- 


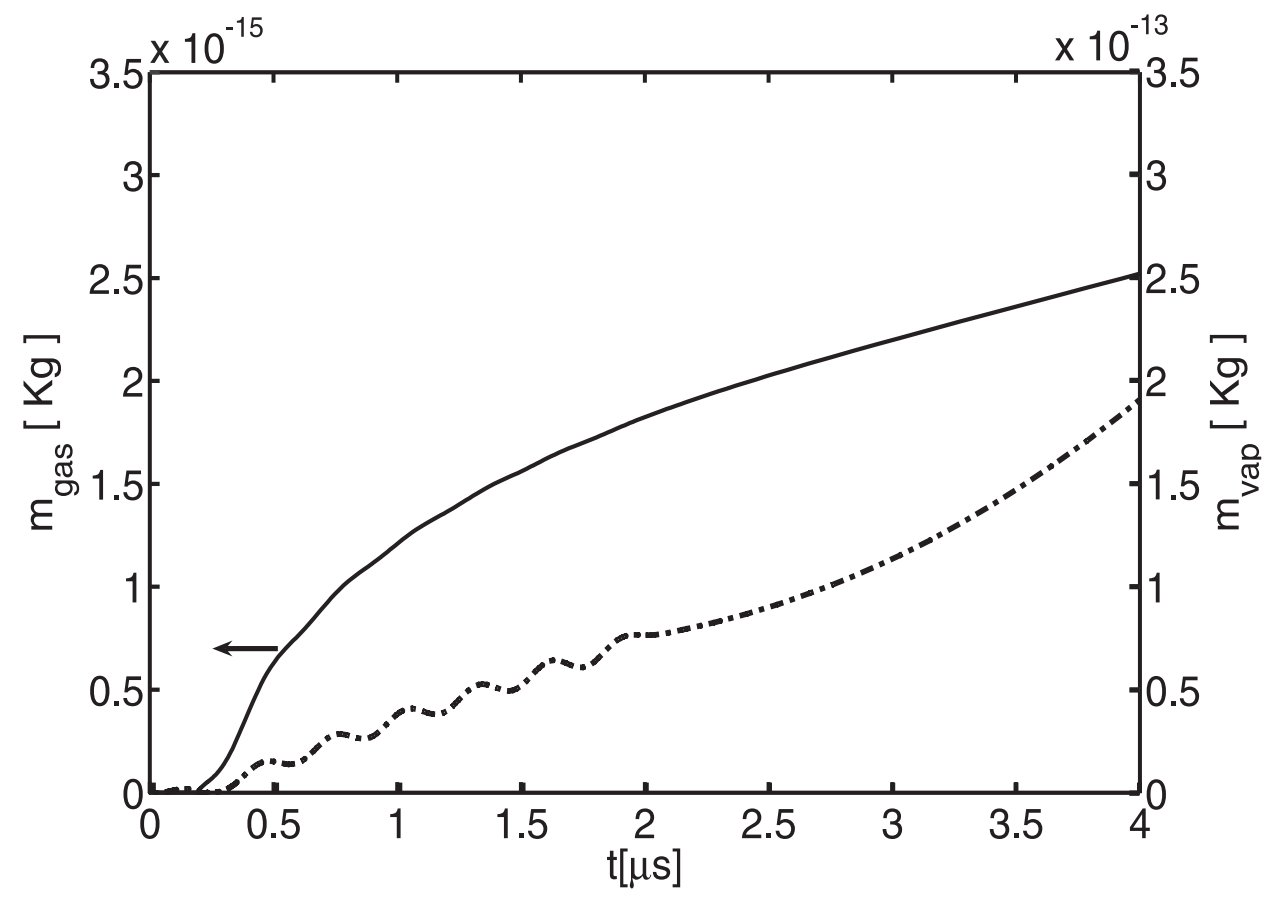

Figure 6.5: Mass of gas (solid) and vapor (dash-dot) inside a PFP bubble versus time, from simulations under the same conditions as in Fig. 6.3. Note the two order of difference between the vertical scales.

cluding gas diffusion, the bubble does not survive the first driving cycle. Simulations with different values of $c_{\infty}$, down to $10 \%$ of the solubility of air in PFP, quantitavely showed the same behavior. Below 10\%, the gas pressure is not high enough to prevent recondensation of the bubble.

The radial dynamics of the vapor bubble is governed by thermal diffusion. Removing the acoustic forcing (dashed line), on the considered time scale, we retrieve the same growth rate found with the acoustic forcing. In this limiting case, including gas diffusion did not have any significant effect on the bubble growth. With no ultrasound affecting the system, from the classical Plesset-Zwick theory [29], we expect a first phase with low growth rate due to surface tension (before $0.2 \mu$ s in Fig.6.4), followed by a linear growth phase dominated by inertia and eventually a squareroot growth dominated by diffusion. Considering the limiting case of purely inertial growth, with a uniform liquid temperature field equal to $T_{\infty}$, from the RayleighPlesset-type equation, assuming $\ddot{R}=0$, it is possible to estimate the radial inertial 
expansion as: $R_{\text {in }} \sim t \sqrt{(2 / 3) \Delta p / \rho_{L}}$, where $\Delta p=p_{\infty}-p_{V, \text { sat }}\left(T_{\infty}\right)$ with $p_{V, \text { sat }}$ imposed by the Clausius-Clapeyron theorem as the pressure of the saturated vapor at $T_{\infty}$. Considering the other limiting case of purely thermal diffusive growth with a uniform pressure field in the liquid equal to $p_{\infty}$, it is possible to estimate from the energy equation the radial thermal expansion as follows: $R_{t h} \sim k_{L} \Delta T /\left(L \rho_{V} \sqrt{D_{L}}\right) \sqrt{t}$ where $\Delta T=T_{\infty}-T_{V, s a t}\left(p_{\infty}\right)$ with $T_{V, \text { sat }}\left(p_{\infty}\right)$ again imposed by the Clausius-Clapeyron theorem as the temperature of the saturated vapor at $p_{\infty}$ [29]. At a specific instant $\bar{t}$, the smallest value of either $R_{t h}$ or $R_{i n}$ is the one that governs the dynamics. At the specific time which we are considering $(\bar{t}=4 \mu \mathrm{s}), R_{t h}$ and $R_{i n}$ are of the same order of magnitude, but with $R_{t h} \lesssim R_{i n}$, we are in a transitional regime between linear and square root-like radial growth.

In Fig. 6.5 we report the mass of gas (solid line) and the mass of vapor (dashdotted line) inside the bubble during the bubble growth. The ratio between the two $m_{G} / m_{V}$ is of order $10^{-2}$, thus justifying the assumptions of the model. The difference between the thermal diffusivity and the mass diffusivity constants, as well as the difference between the temperature gradient and the concentration gradient driving the two diffusive phenomena, leads to different time scales and therefore to different shapes of the curves $m_{G}(t)$ and $m_{V}(t)$.

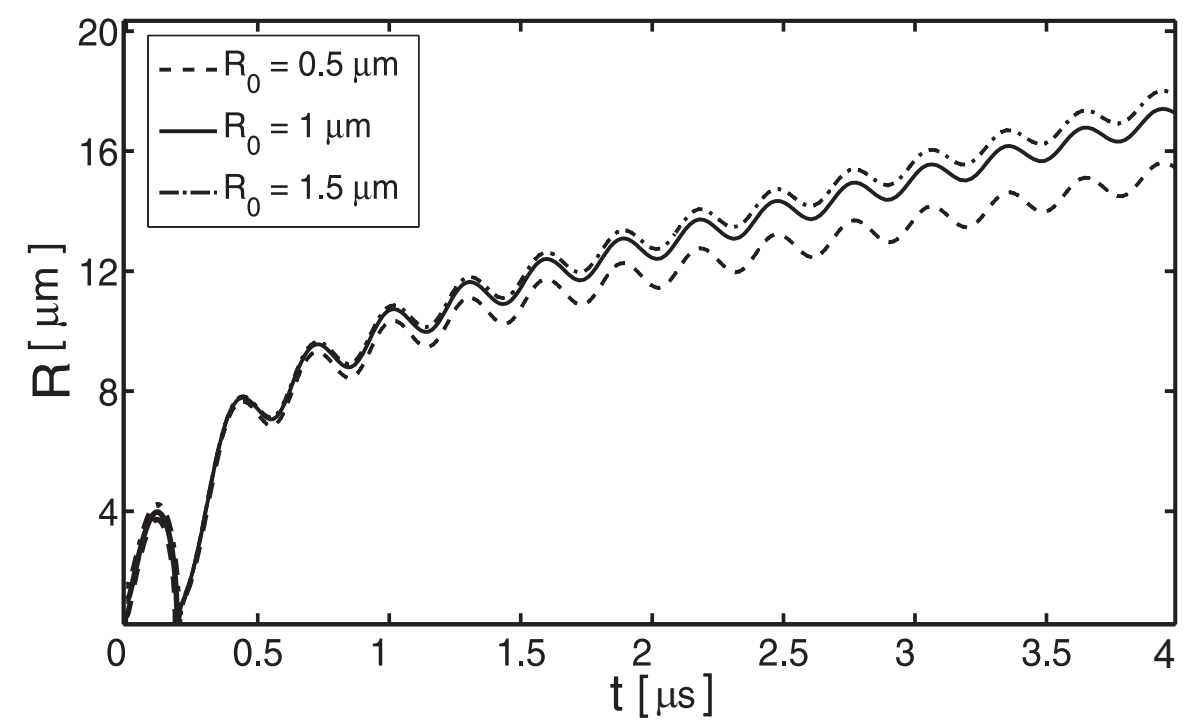

Figure 6.6: Radius-time curve $R(t)$ from simulations for a PFP bubble for different initial radii $R_{0}$, at $15^{\circ} \mathrm{C}$ superheat, driven at frequency $f=3.5 \mathrm{MHz}$, pressure $P_{a}=$ 4.5 MPa. 


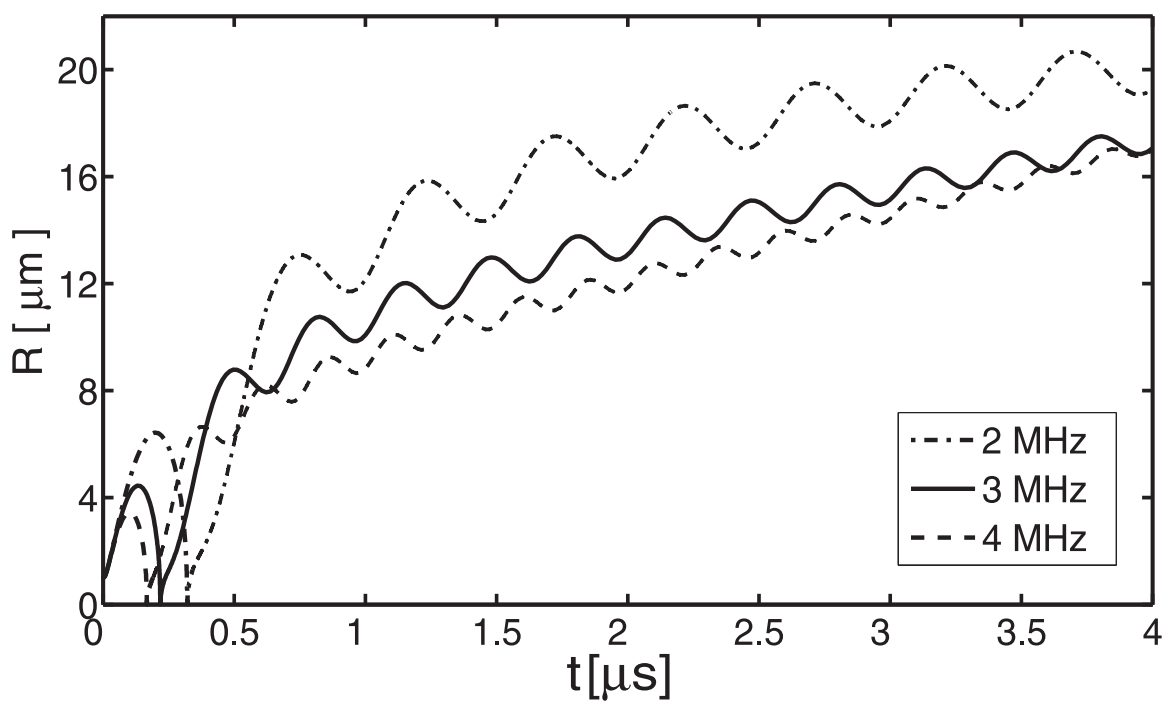

Figure 6.7: Radius-time curve $R(t)$ from simulations for a $1 \mu \mathrm{m}$ PFP bubble driven at different frequencies with pressure $P_{a}=4.5 \mathrm{MPa}$, at $15^{\circ} \mathrm{C}$ superheat.

In order to verify the robustness of predictions with respect to the initial radius chosen, we performed several calculations, varying $R_{0}$ (see Fig. 6.6). A change of this parameter by $50 \%$ in either way relative to the adopted value of $1 \mu \mathrm{m}$, inferred from the first available data point, resulted only into a small change of the growth rate of the bubble: after the first collapse it is between $+8 \%$ and $-20 \%$. The amplitude of the bubble oscillations was left unperturbed. Therefore we can conclude that the simulations are robust with respect to the choice of the initial radius.

We also explored the effects of the driving frequency on the bubble dynamics (see Fig. 6.7). At the relevant time scales, lower frequencies corresponded both to larger oscillations and larger average values of the radius. Larger oscillations at low frequencies are due to the fact that the bubble has more time to expand. Enhancement of rectified heat transfer [30-32] due to this large oscillation amplitude magnification can explain the faster growth rates at the initial stages (after $3 \mu \mathrm{s}$ ) at lower frequencies. However, the associated growth rates reverse their frequency dependence at later stages (after three microseconds). 


\subsection{Summary and conclusions}

In the presented work we follow the growth dynamics of a PFP vapor bubble nucleated and driven with ultrasound, inside a PFP droplet, contained in a water medium superheated with respect to the PFP boiling point. The model we introduced here, describing the evolution of a ultrasound driven vapor bubble inside a superheated infinite medium of the same substance, succeeded to reproduce the dynamics of such a bubble within a $10 \%$ error. It is important to note that our model does not contain any free fitting parameters. It included both thermal diffusion and mass diffusion of gas (air) inside the liquid. Simulations underlined the fundamental role of gas diffusion during acoustic forcing. Though the bubble dynamics is entirely governed by evaporation and condensation processes, as the rate between gas and vapor mass is $m_{G} / m_{V} \sim 10^{-2}$, a minimum amount of gas $(\sim 10 \%$ of the air solubility in PFP) is necessary in order to allow the bubble to survive the first collapse. When no driving is present, including or neglecting gas diffusion has no influence on the bubble growth. The analysis of this limiting case showed that, at time scales considered, we are in a transition region, in which thermal diffusion becomes more relevant than inertia.

Given the difficulties of experimentally monitoring the initial nucleation phase of the vapor bubble, we examined the role of the initial nucleation radius on the bubble dynamics by means of simulations. Larger initial nucleation radii lead to larger growth rates, although the oscillation amplitude is left unperturbed. We found that a change of $\pm 50 \%$ in $R_{0}$ induces a change of the bubble growth rate after the first collapse, within $30 \%$ of its original value. We also examined the effect of the driving frequency, and found that lower frequencies lead to larger oscillation amplitudes and larger bubble sizes at the relevant time scales. 


\section{References}

[1] N. Y. Rapoport, Z. Gao, and A. Kennedy, "Multifunctional nanoparticles for combining ultrasonic tumor imaging and targeted chemotherapy", J. Natl. Cancer. Inst. 99, 1095-1106 (2007).

[2] P. Mohan and N. Rapoport, "Doxorubicin as a molecular nanotheranostic agent: Effect of doxorubicin encapsulation in micelles or nanoemulsions on the ultrasound-mediated intracellular delivery and nuclear trafficking.", Mol. Pharmaceut. 7, 1959-1973 (2010).

[3] O. D. Kripfgans, C. Orifici, P. Carson, K. A. Ives, O. P. Eldevik, and J. B. Fowlkes, "Acoustic droplet vaporization for temporal and spatial control of tissue occlusion: a kidney study", IEEE 52, 1101-1110 (2005).

[4] M. Zhang, M. L. Fabiilli, K. J. Haworth, F. Padilla, S. D. Swanson, O. D. Kripfgans, P. Carson, and J. Fowlkes, "Acoustic droplet vaporization for enhancement of thermal ablation by high intensity focused ultrasound", Acad Radiol. 18, 1123-1132 (2011).

[5] P. S. Sheeran, V. P. Wong, S. Luois, R. J. McFarland, W. D. Ross, S. Feingold, T. Matsunaga, and P. Dayton, "Decafluorobutane as a phase-change contrast agent for low-energy extravascular ultrasonic imaging.", UMB 37, 1518-1530 (2011).

[6] O. Couture, M. Faivre, N. Pannacci, A. Babataheri, V. Servois, P. Tabeling, and M. Tanter, "Ultrasound internal tattooing", Med. Phys 38, 1116-1123 (2011).

[7] N. Reznik, R. Williams, , and P. N. Burns, "Investigation of vaporized submicron perfluorocarbon droplets as an ultrasound contrast agent", UMB 37, 1271-1279 (2011).

[8] Z. Wong, O. Kripfgans, A. Qamar, J. Fowlkes, and J. L. Bull, "Bubble evolution in acoustic droplet vaporization at physiological temperature via ultra-high speed imaging", Soft Matter 7, 4009-4016 (2011).

[9] M. Fabiilli, J. Lee, O. Kripfgans, P. Carson, and J. Fowlkes, "Delivery of watersoluble drugs using acoustically triggered perfluorocarbon double emulsions", Pharm. Res. 27, 2753-2765 (2010).

[10] A. Qamar, Z. Z. Wong, J. B. Fowlkes, and J. L. Bull, "Dynamics of acoustic droplet vaporization in gas embolotherapy", Appl. Phys. Lett. 96, 143702 (2010). 
[11] R. I. Nigmatulin and N. S. Khabeev, "Dynamics of vapour-gas bubbles", Izvestiya Akademii Nauk SSSR, Mekhanika Zhidkosti i Gaza 56-61 (1976).

[12] R. I. Nigmatulin, N. S. Khabeev, and F. B. Nagiev, "Dynamics, heat and mass transfer of vapour-gas bubbles in a liquid", Int. J. Heat Mass Transfer 24, 10331044 (1981).

[13] M. Fanelli, A. Prosperetti, and M. Reali, "Radial oscillations of gas-vapor bubbles in liquid. pt. 1. mathematical formulation", Acoustica 253-265 (1981).

[14] N. S. Khabeev, "Radial oscillations of vapor-gas bubbles", J. Appl. Mech. Tech. Phys. 6 (1987).

[15] N. S. Khabeev, "Diffusion effects in the oscillation of vaporgas bubbles in a sound field", Int. J. Heat Mass Tran. 50, 3556-3560 (2007).

[16] G. Dassie and M. Reali, "Dynamics of an oscillating spherical gas/vapor bubble", J. Acoust. Soc. Am. 100, 3088-3097 (1996).

[17] M. Al-Monnai and N. S. Khabeev, "Radial oscillations of soluble vapor-gas bubbles in a liquid”, Fluid. Dyn. 46, 286-290 (2011).

[18] M. Fabiilli, K. Haworth, N. Fakhri, O. Kripfgans, P. Carson, and J. Fowlkes, "The role of inertial cavitation in acoustic droplet vaporization", IEEE Trans. Ultrason. Ferroelec. Freq. Contr. 56, 1006-1017 (2009).

[19] O. Shpak, T. Kokhuis, Y. Luan, D. Lohse, N. de Jong, B. Fowlkes, M. Fabiilli, and M. Versluis, "Ultrafast dynamics of the acoustic vaporization of phasechange microdroplets", J. Acoust. Soc. Am. (submitted, 2013).

[20] Y. Hao and A. Prosperetti, "The dynamics of vapor bubbles in acoustic pressure fields", Phys. Fluids 11, 2008-2019 (1999).

[21] B. Yang and A. Prosperetti, "Vapor bubble collapse in isothermal and nonisothermal liquids", J. Fluid Mech. 601, 253 - 279 (2008).

[22] L. Stricker, A. Prosperetti, and D. Lohse, "Validation of an approximate model for the thermal behavior in acoustically driven bubbles", J. Acoust. Soc. Am. 130, 3243-3251 (2011).

[23] W. Sutherland, "A dynamical theory of diffusion for non-electrolytes and the molecular mass of albumin", Phil. Mag. 9, 781-785 (1905). 
[24] J. Loschmidt, "Zur größe der luftmoleküle", Sitzungsberichte der kaiserlichen Akademie der Wissenschaften Wien 52, 395-413 (1865).

[25] National Institute of Standards and Technology, "NIST repository", http:// wtt-pro.nist.gov/wtt-pro/\#perfluoropentane (2012).

[26] 3M, "Fluorinert liquids for electronics manufacturing, product information", http://multimedia. $3 \mathrm{~m} \cdot \mathrm{com} / \mathrm{mws} / \mathrm{mediawebserver?mwsId=}$ SSSSSufSevTsZxtUM8tZ18_9evUqevTSevTSevTSeSSSSSS--\&fn= prodinfo_fluorinert.pdf (2012).

[27] V. Kamath and A. Prosperetti, "Numerical integration methods in gas-bubble dynamics", J. Acoust. Soc. Am. 85, 1538 (1989-1548).

[28] T. D. Martz, P. S. Sheeran, D. Bardin, A. P. Lee, and P. A. Dayton, "Precision manufacture of phase-change perfluorocarbon droplets using microfluidics”, Ultrasound Med. Biol. 37, 1952-1957 (2011).

[29] M. S. Plesset and S. A. Zwick, "The growth of vapor bubbles in superheated liquids", J. Appl. Phys. 25, 493-499 (1954).

[30] G. Trammell, "Sound waves in water containing vapor bubbles", J. Appl. Phys. 33, 1662-1670 (1962).

[31] R. Finch and E. Neppiras, "Vapor bubble dynamics", J. Acoust. Soc. Am. 53, 1402-1410 (1973).

[32] T. Wang, "Rectified heat transfer", J. Acoust. Soc. Am. 56, 1131-1143 (1974). 


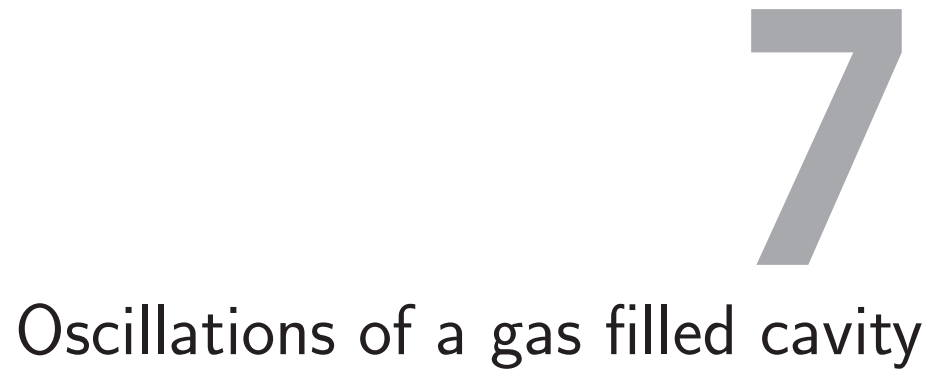

In some kinds of last generation efficient sonochemical reactors, the creation of bubbles is enhanced by means of ultrasonic activation of artificial silicon-etched micropits. In the present chapter, we study the oscillations of a gas pocket entrapped inside a cylindrical micropit, by means of a combined level set and projection method approach. We compare our results with the analytical ones for small oscillations in the limiting cases of Stokes and potential flow, finding good agreement. We extend the study to the intermediate regime where both viscosity and advection are important and where no analytical study has been done until now. We examine both the small and large amplitude free oscillations, as well as the effects of the presence of a rigid wall. We also consider the forced oscillations, showing that, when the flat interface is taken as equilibrium condition, even complex initial perturbations relax in few acoustic cycles when the air pocket is forced at its main resonant frequency. This suggests that, despite the abruptness of the response, the bubble formation mechanism is something other than the main resonance.

\subsection{Introduction}

Gas pockets of the order of some microns can be stabilized against diffusion by enclosure within small rigid structured and crevices, such as pits and pores [1]. The ultrasonic activation of these entrapped pockets is associated to several phenomena, 
such as bubble nucleation [2, 3], small-scale motion (microstreaming) [4, 5], collection of suspend particles at the active crevices [4, 6] and mixing [7]. In biological systems, these phenomena can result into severe damage. In plant leaves [8] and insects [9] cell disruption and death have been observed as a consequence of the ultrasonic activation of entrapped gas pockets; in humans, the rupture of platelets [10] and erythrocytes [11] and the accumulation of red cells around acoustically activated holes [4] have been reported. These phenomena can also be exploited for practical applications. The microstreaming generated by the pulsations of the entrapped bubble has been used to study the consequences of shear stress upon bacteria and cells $[12,13]$, while the disruptive potential of pore-originated cavitation can facilitate drug delivery [14] and gene transfection [15]. Moreover, controlled bubble growth and collapse can be achieved through patterned membranes [16] and silicon etched micropits $[2,3,17]$. In particular, this last technology found recent application in the fabrication of efficient sonochemical reactors [18, 19].

Even if the technological applications are manifold, the dynamics of a single oscillating gas pocket is still a problem that needs to be investigated. Few experimental works are available on the dynamics of ultrasonically driven entrapped bubbles, and they mainly deal with patterned surfaces and multiple bubbles [16, 20-22]. Though Rathgen et al. [21] measured the maximum deflection of the menisci during acoustic oscillation, they could not resolve the full shape of the interface.

Some theoretical studies have been published, where the entrapped bubble dynamics was studied under restrictive assumptions. In Refs. [21, 23], the authors calculated its resonance frequency within the assumption that the interface remained parabolic. An analytic approach has been recently developed from Gelderblom et $a$. [24], in the case of small amplitude oscillations, for the limiting cases of potential and Stokes flow.

The objective of the present work is to understand the dynamic behavior of an entrapped bubble inside a cylindrical crevice of the kind described in Chap. 3 and Ref. [19], without any of these assumptions. To this aim, we adopt a numerical approach that allows us to take into account both advection and viscosity. The method is based on a level set approach, combined with a staggered grid projection method $[25,26]$. A vast literature is available for level set methods, including some books $[27,28]$. For a review, we refer the reader to Ref. [29]. These methods have proved particularly efficient when singularities and contact lines are present [30-32]. After validating the code by comparing the numerical results with the analytical ones in the limiting cases of Stokes and potential flow for small amplitude oscillations, we examine the intermediate regime where both advection and viscosity are present, and the large amplitude free and forced oscillations. We also consider the effect of the 


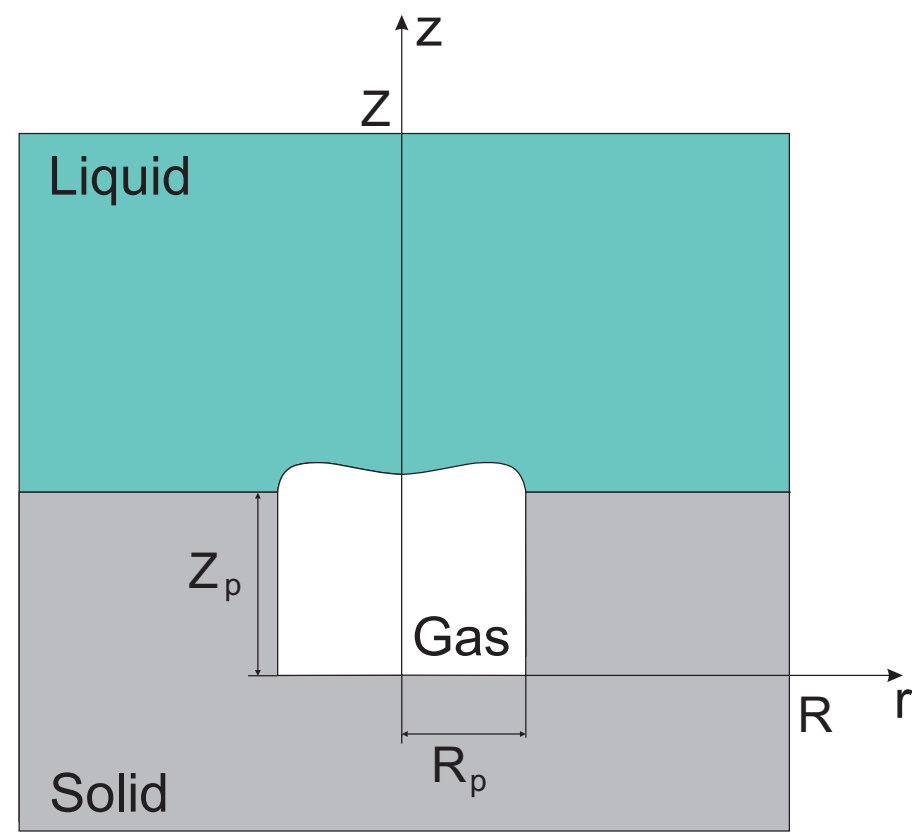

Figure 7.1: Sketch and nomenclature for the micropit containing the entrapped bubble.

presence of a solid boundary near the pit, which is a condition occurring in cleaning devices $[33,34]$.

\subsection{Model}

We consider a cylindrical pit etched on a flat plate immersed inside a liquid and filled with air (see Fig. 7.1). Several assumptions are made in the following. We assume that the meniscus is pinned at the edge of the pit. The liquid is taken as incompressible. The gas is regarded as ideal and we neglect both its viscosity and inertia, because it has a lower density with respect to the liquid. Therefore the gas pressure is considered spatially uniform. These are reasonable assumptions as the Mach numbers are small $(M a<<1)$, i.e. the velocities are small compared to the sound velocity. The temperature of the gas is also assumed to be spatially uniform and it is kept constant in time. We assume that the system is axially symmetric and we adopt cylindrical coordinates.

We study the evolution of the velocity field $\mathbf{u}(t, r, z)$ and the pressure field $p(t, r, z)$ 
in the liquid, where $t$ is the time, $r$ is the radial coordinate and $z$ is the axial coordinate. The motion of the liquid is described by the Navier-Stokes equations. In the case of forced oscillations, an acoustic oscillating driving pressure is applied on the free boundaries of the computational domain, ideally at $r \rightarrow \infty$.

The equations expressing the conservation of mass and momentum are

$$
\begin{gathered}
\nabla \cdot \mathbf{u} \\
\frac{\partial \mathbf{u}}{\partial t}+\mathbf{u} \cdot \nabla \mathbf{u}=-\frac{1}{\rho} \nabla p+\frac{\mu}{\rho} \nabla^{2} \mathbf{u},
\end{gathered}
$$

in which $\mu$ and $\rho$ are the viscosity and the density of the liquid, respectively.

At the interface, a balance of normal and tangential stresses holds. Since the gas viscous stress is neglected, the liquid tangential stress is null:

$$
\mathbf{t} \cdot \mathbf{D} \cdot \mathbf{n}=0
$$

where $\mathbf{n}$ and $\mathbf{t}$ are the unit normal and tangential vectors at the interface respectively, and $\mathbf{D}=\mu\left(\nabla \mathbf{u}+\nabla \mathbf{u}^{T}\right)$ is the stress tensor. The normal stresses balance can be expressed as

$$
p_{G}=p_{B}+\sigma \kappa-\mathbf{n} \cdot \mathbf{D} \cdot \mathbf{n},
$$

where $p_{G}$ is the gas pressure, $p_{B}$ is the pressure at the interface on the liquid side, $\sigma$ is the surface tension and $\kappa$ is the local curvature. Eq. (7.3) and (7.4) provide boundary conditions at the interface for velocity and pressure.

The pressure of the gas follows the perfect gas law

$$
p_{G} V^{K}=p_{0} V_{0}^{K}
$$

in which $V$ is the volume of the entrapped bubble, $K=1$ as we consider an isothermal process, $p_{0}$ is the ambient pressure of the liquid and $V_{0}$ is the geometric volume of the pit.

The pressure of the liquid at infinity is given by

$$
p_{\infty}=p_{0}+P_{a} \sin (\omega t)
$$

in which $P_{a}$ is the driving amplitude and $\omega=2 \pi f$ with $f$ the driving frequency.

\subsection{Numerical method}

In order to track the position of the gas/liquid interface, we use a level set method coupled to a projection method. The latter is used to determine the velocity and 
pressure fields in the liquid. We refer the reader to Refs. [27, 28] for an extensive overview of the level set method and to Refs. [25, 26] for details on the numerical treatment adopted in the present work. A summary is given in the following, with particular care for the singularity encountered at the contact line, where the meniscus is pinned to the edge of the pit. We consider a computational domain of dimensions $R \times Z$ (see Fig. 7.1) with $N_{R} \times N_{Z}$ cells and a pit with dimensions $R_{p} \times Z_{p}$ and $N_{R \text { pit }} \mathrm{x} N_{Z \text { pit }}$ cells. The method requires the introduction of a smooth function $\Phi(\mathbf{x})$, called the level set function, defined at the center of each computational cell. The gas phase is identified by $\Phi<0$, while the liquid phase is characterized by $\Phi>0$. The set of points $\mathbf{x}$ where $\Phi=0$ corresponds to the interface. In order to simplify the equations resulting in the numerical treatment, $\Phi$ is chosen with the particular property of being a signed distance function, i.e. a function whose value at point $\mathbf{x}$ is equal to the shortest distance between $\mathbf{x}$ and the interface

$$
\Phi(\mathbf{x})=S(\Phi(\mathbf{x})) \min \left(\left|\mathbf{x}-\mathbf{x}_{\Gamma}\right|\right), \quad \forall \mathbf{x}_{\Gamma} \in \Gamma .
$$

Here $\mathbf{x}_{\Gamma}$ is a point on the interface and $S(\Phi)$ is the sign of the level set function, defined as

$$
S(\Phi)= \begin{cases}1, & \text { if } \Phi>0 \\ 0, & \text { if } \Phi=0 \\ -1, & \text { if } \Phi<0\end{cases}
$$

For example, when the interface is a $2 \mathrm{D}$ circle of radius $R$ centered at $\left(x_{0}, y_{0}\right)$, the level set function will be the cone $\Phi(x, y)=\sqrt{\left(x-x_{0}\right)^{2}+\left(y-y_{0}\right)^{2}}-R$. In our case, a graphic representation of the full level set function is not possible, as the interface is $3 \mathrm{D}$ and $\Phi$ is $4 \mathrm{D}$. However, as we assume axial symmetry, $3 \mathrm{D}$ contours of the level set can be shown, for a fixed meridian plane (see Fig. 7.2).

At any point of the interface, the unit normal vector $\mathbf{n}$ and the local (3D) curvature $\kappa$ can be found through

$$
\begin{aligned}
& \mathbf{n}=\frac{\nabla \Phi}{|\nabla \Phi|}, \\
& \kappa=-\nabla \cdot \mathbf{n}
\end{aligned}
$$

The level set function is advected by means of the equation

$$
\frac{\partial \Phi}{\partial t}+\mathbf{u} \cdot \nabla \Phi=0
$$

in which, $\mathbf{u}$ is the velocity of the fluid at point $\mathbf{x}$, either real (where $\Phi>0$ ) or extrapolated (where $\Phi<0$ ). The extrapolation of the velocity field is a delicate issue and we will address it later. After advecting the level set, a correction of $\Phi$ becomes 


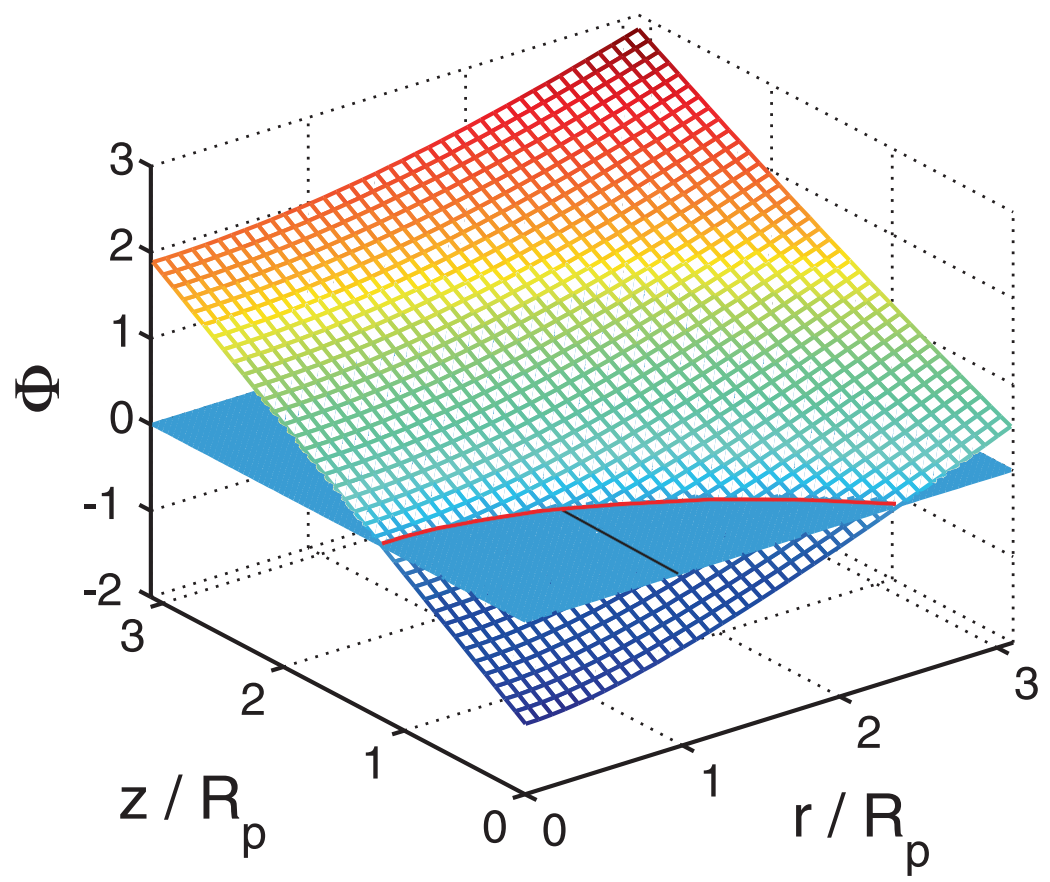

Figure 7.2: Example of 3D contour of a 4D level set line used to represent the meniscus (red), pinched at the pit edge (black).

necessary in order to enforce the signed-distance property. This manipulation, called reinitialization, must not displace the interface and it can be performed in different ways [35-37]. As a direct calculation of the zero level set [35] is computationally expensive, in the present work we adopt the method of Ref. [36]. We solve the equation

$$
\begin{aligned}
\frac{\partial \Phi}{\partial \tau}+S\left(\Phi^{0}\right)(1-|\nabla \Phi|) & =0 \\
\Phi(\mathbf{x}, 0) & =\Phi^{0}
\end{aligned}
$$

until steady state is reached. Here $\tau$ is an artificial time and $\Phi^{0}$ is the level set function calculated with (7.11). This procedure is based on the property that a signed distance function $\Phi$ has $|\nabla \Phi|=1$ at all points except those equidistant from two or more points of the interface. This singularity region, where the gradient is not rigorously defined, is usually called 'the skeleton'. In the present work we take care of reducing its dimension by regularizing $\Phi$ at the contact line, in order to avoid the development of numerical instabilities. We extend $\Phi$ in the solid region in a smooth fashion, by 
means of the osculating paraboloid through the contact line. We enforce the zero level set of such extension to have the same tangent plane and 3D curvature as the existing interface at the point where the meniscus is pinned to the pit edge (see Fig. 7.2 and Fig. 7.15). In the numerical treatment of Eq. (7.12), the sign function is substituted by a smeared Heaviside function

$$
H(\Phi)= \begin{cases}1, & \text { if } \Phi>\varepsilon \\ \frac{1}{2}+\frac{\Phi}{2 \varepsilon}+\frac{1}{2 \pi} \sin \frac{\pi \Phi}{\varepsilon}, & \text { if }-\varepsilon<\Phi<\varepsilon \\ -1, & \text { if } \Phi<\varepsilon\end{cases}
$$

to prevent numerical instabilities.

The level set advection equation (7.11) is integrated in time by means of an Adam-Bashforth scheme

$$
\frac{\Phi^{n+1}-\Phi^{n}}{\Delta t}=-\frac{3}{2}(\mathbf{u} \cdot \nabla \Phi)+\frac{1}{2}(\mathbf{u} \cdot \nabla \Phi)^{n-1},
$$

in which the superscript indicates the time instant. The spatial derivatives of $\Phi$ are computed with a fifth order WENO scheme [38].

The reinitialization equation (7.12) is integrated in the pseudo-time $\tau$ with the third order Runge-Kutta scheme described in [39], consisting of a linear combination of Euler integration steps:

$$
\begin{aligned}
\Phi^{(1)} & =\Phi^{n}-\Delta t L \Phi^{n} \\
\Phi^{(2)} & =\frac{3}{4} \Phi^{n}+\frac{1}{4} \Phi^{(1)}-\frac{1}{4} \Delta t L \Phi^{(1)} \\
\Phi^{n+1} & =\frac{1}{3} \Phi^{n}+\frac{2}{3} \Phi^{(2)}-\frac{2}{3} \Delta t L \Phi^{(2)}
\end{aligned}
$$

with $L \Phi^{(k)}=S\left(\Phi^{0}\right)\left[\left|\nabla \Phi^{(k)}\right|-1\right]$. Here the spatial derivatives of $\Phi$ are computed using the "subcell fix" method described in Ref. [40], in order to prevent mass loss and growth of oscillations [25]. With this method, spatial derivatives of $\Phi$ at point $\mathbf{x}$ are computed with a fifth order WENO scheme, when $\mathbf{x}$ is far from the interface, and with a third order modified ENO scheme when $\mathbf{x}$ is close to the interface. This procedure is essential to guarantee the upwind nature of the scheme, in agreement with the fact that the reinitialization equation is a hyperbolic equation whose characteristics always propagate from the interface to the liquid [25].

The equations of momentum (7.2) and mass conservation (7.1) are solved by means of a projection method [41]. We use a standard staggered grid for the pressure and the velocity fields. The momentum equation (7.2) is integrated by means of 


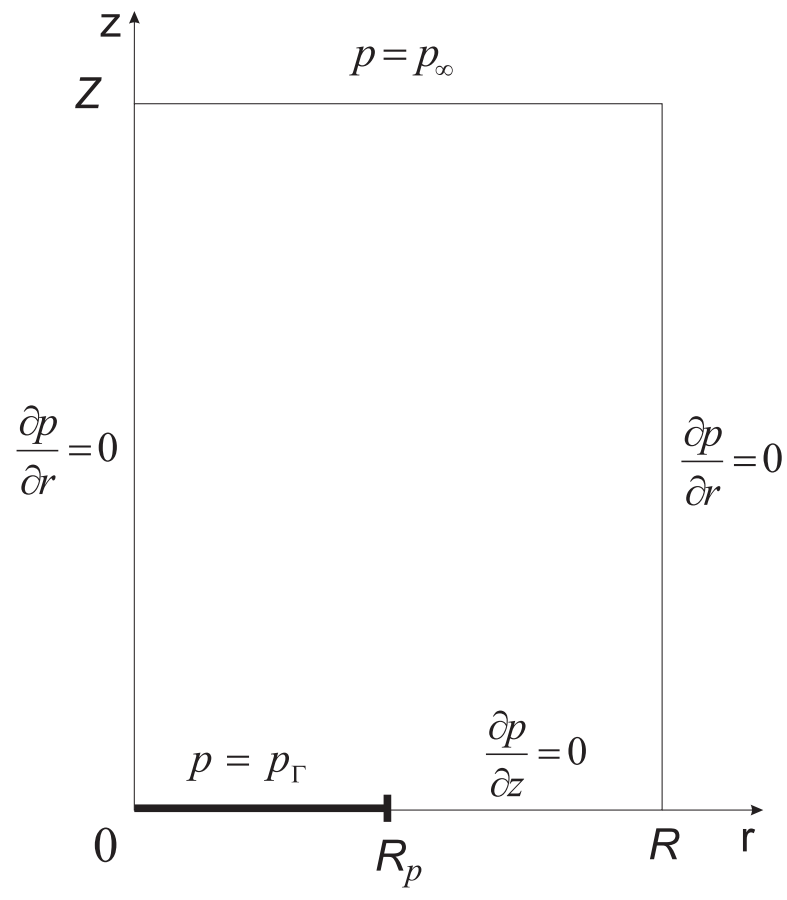

Figure 7.3: Sketch of the boundary condition for the mixed boundary Laplace problem used as test case for the pressure field in Sec.7.4.

two separate steps, by the introduction of an intermediate velocity field $\mathbf{u}^{\star}$. The first intermediate step consists of a predictor step, where the advective and diffusive terms are treated explicitly and $\mathbf{u}^{\star}$ is found with a first order Euler method:

$$
\frac{\mathbf{u}^{\star}-\mathbf{u}^{n}}{\Delta t}=-(\mathbf{u} \cdot \nabla \mathbf{u})^{n}+\frac{1}{\rho}(\nabla \cdot \mathbf{D})^{n} .
$$

As $\mathbf{u}^{\star}$ is not divergence-free, a projection step is required, to find the real field $\mathbf{u}^{n+1}$ :

$$
\frac{\mathbf{u}^{n+1}-\mathbf{u}^{\star}}{\Delta t}=-\frac{\nabla p^{n+1}}{\rho} .
$$

In order to calculate the pressure field $p$, we apply the divergence operator to both sides of (7.17), imposing the desidered property that the final velocity field is divergencefree: $\nabla \cdot \mathbf{u}^{n+1}=0$ thus obtaining the Poisson equation

$$
\frac{1}{\rho} \nabla^{2} p^{n+1}=\frac{\nabla \cdot \mathbf{u}^{\star}}{\Delta t} .
$$


This equation has to be solved subject to the boundary conditions: $p=p_{\Gamma}$ at the liquid/gas interface, $\left.\frac{\partial p}{\partial r}\right|_{r=0}=0$ at the $\mathrm{z}$-axis (left boundary) for symmetry reasons, $\left.\frac{\partial p}{\partial z}\right|_{z=Z_{p}, r>R_{p}}=0$ at the bottom, $p=p_{\infty}$ at the top and right boundaries, for open flow conditions or $\frac{\partial p}{\partial n}=0$ for rigid wall conditions. In the integration of (7.16), the spatial derivatives of $\mathbf{u}^{n}$ are calculated by means of a fifth order ENO scheme. In order both to impose the boundary conditions on the velocity field when solving (7.16) and to advect the interface in the proper way by means of (7.11) without generating numerical instabilities, we extrapolate the liquid velocity field inside the gas. This step results particularly delicate because the extrapolated field has to satisfy also the incompressibility constraint in order to guarantee an accurate and mass-preserving displacement of the interface [42]. In order to achieve this, we use a technique similar to the one described in Ref. [25], adapting it to the specific case. Under the surface of the rigid horizontal boundary at $z=Z_{p}$, we introduce 3 rows of ghost cells through a mirroring procedure in such a way that, when the fifth order ENO gradient is calculated, the velocities satisfy both the no-slip condition and the incompressibility constraint. The velocity is then extrapolated using the Lagrangian multipliers method described in Ref. [25], both in the gas and in the solid substrate around the pit, within a band of 6 cells from the liquid-gas interface and from the horizontal liquid-solid interface respectively. We impose both the incompressibility constraint and the free-slip condition at the gas-liquid interface in the Lagrangian function. Although, theoretically, this should be enough to ensure the divergence-free constraint, near the contact line the $\nabla \cdot \mathbf{u}$ can still be non-zero. Therefore, a further projection step in the extrapolated velocity field is required, similar to the one adopted to ensure incompressibility in the real velocity field [25]. The 3 rows of ghost cells, adjacent to the horizontal solid-liquid interface at $z=Z_{p}$, are excluded from this projection step.

\subsection{Validation}

In order to validate the liquid pressure field calculated from the Poisson equation (7.18), we compared the numerical results with the analytical solution in a test case with flat meniscus, velocity equals zero everywhere and vertical rigid wall coaxial to the pit at the right boundary. The boundary conditions were imposed as in Fig. 7.3, in order to match the boundary conditions used in the projection method for the pressure, thus giving origin to a mixed boundary problem. The analytical solution of this problem is derived in Appendix A. We found good agreement between the numerical and the analytical results (see Fig. 7.4). We also verified that convergence was reached by increasing the number of cells of the computational domain (see Fig. 7.5). 
In order to validate the full dynamics, we considered the limiting cases of Stokes flow and potential flow for small amplitude oscillations and we compared the numerical results with the analytic solution calculated in Ref. [24]. To reproduce the Stokes flow conditions, we set to zero the advective term inside (7.16) and we imposed a no-slip condition both at the gas-interface and at the solid wall. For the potential flow, in order to fulfill the zero-vorticity requirement, we set to zero the viscous term in (7.16), we imposed the free slip condition at the solid wall and the zero-vorticity constraint itself at the gas-liquid interface, in the Lagrangian function used to find the extrapolated velocity field. We verified that, since the vorticity vanished at the boundaries, no vorticity was present in the rest of the domain as well, as expected [43]. Both for the Stokes and the potential flow, we gave an initial perturbation to the gas-liquid interface, i.e. we 'pulled' the meniscus and we let it oscillate freely. The

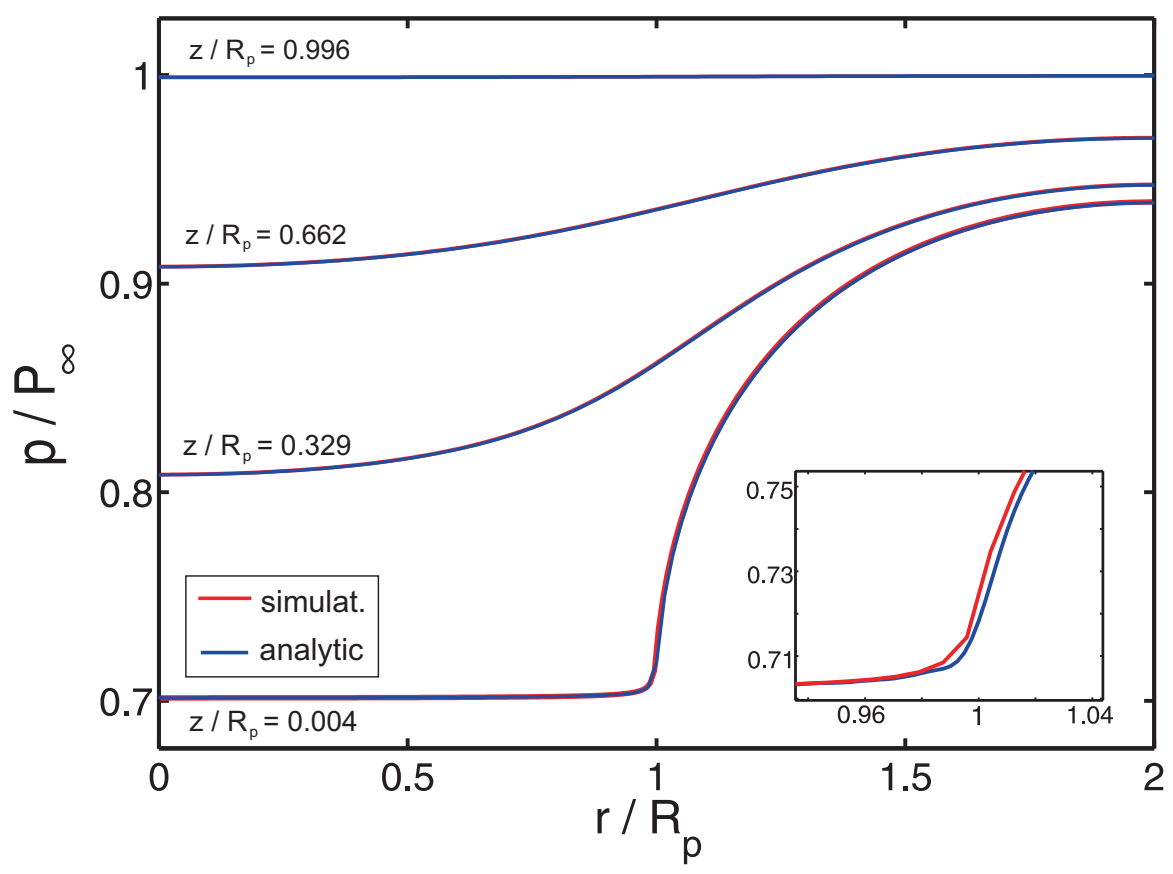

Figure 7.4: Comparison of the analytical (blue) and numerical (red) pressure field in the test case of Sec.7.4, at fixed $z$. Parameters: $R_{p}=R / 2=Z, N_{R}=240$. The pit here corresponds to the negative values of $z$. 


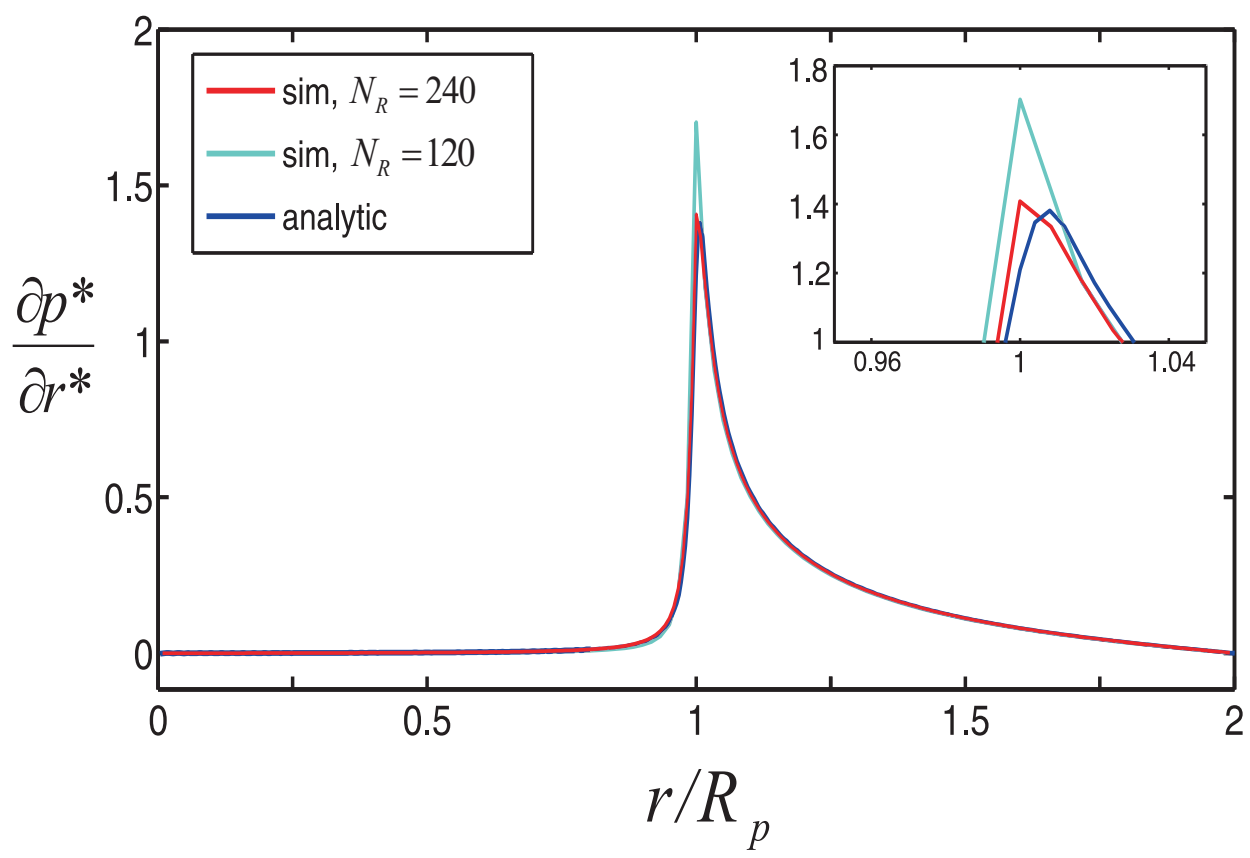

Figure 7.5: Comparison of the analytical (blue) and numerical non-dimensional gradient of the pressure $\partial p * / \partial r *$ at $z / R_{p}=0.0125$, in the test case of Sec.7.4, for different number of cells (as indicated in the legend). The adimensional quantities are defined as $p *=p / P_{\infty}, r *=r / R_{p}$. Parameters: $R_{p}=R / 2=Z$. The pit here corresponds to the negative values of $z$.

initial perturbation had the shape of a paraboloid with the vertex on the $z$ axis:

$$
z=\frac{Z_{p}-z_{0}}{R_{p}^{2}} r^{2}+z_{0}
$$

where $z_{0}=Z_{p}+\Delta z_{0}$, such that $\Delta z_{0} \ll Z_{p}$. In Fig. 7.6.a and in Fig. 7.7 we show the free oscillations of the central point of the meniscus for the Stokes and the potential flows, respectively.

For Stokes flow, we derived the resonance frequency $f_{0}$ of the first mode from the distance between two consecutive peaks on the $t$ axis, while for potential flow we used Fourier analysis. For Stokes flow we also calculated the damping coefficient $\beta$ of the first mode by fitting a decaying exponential through the peaks of the $z(r=0, t)$ curve. As the initial perturbation did not correspond exactly to the first mode, the contribution of some other modes was also present in the initial oscillations. Therefore 

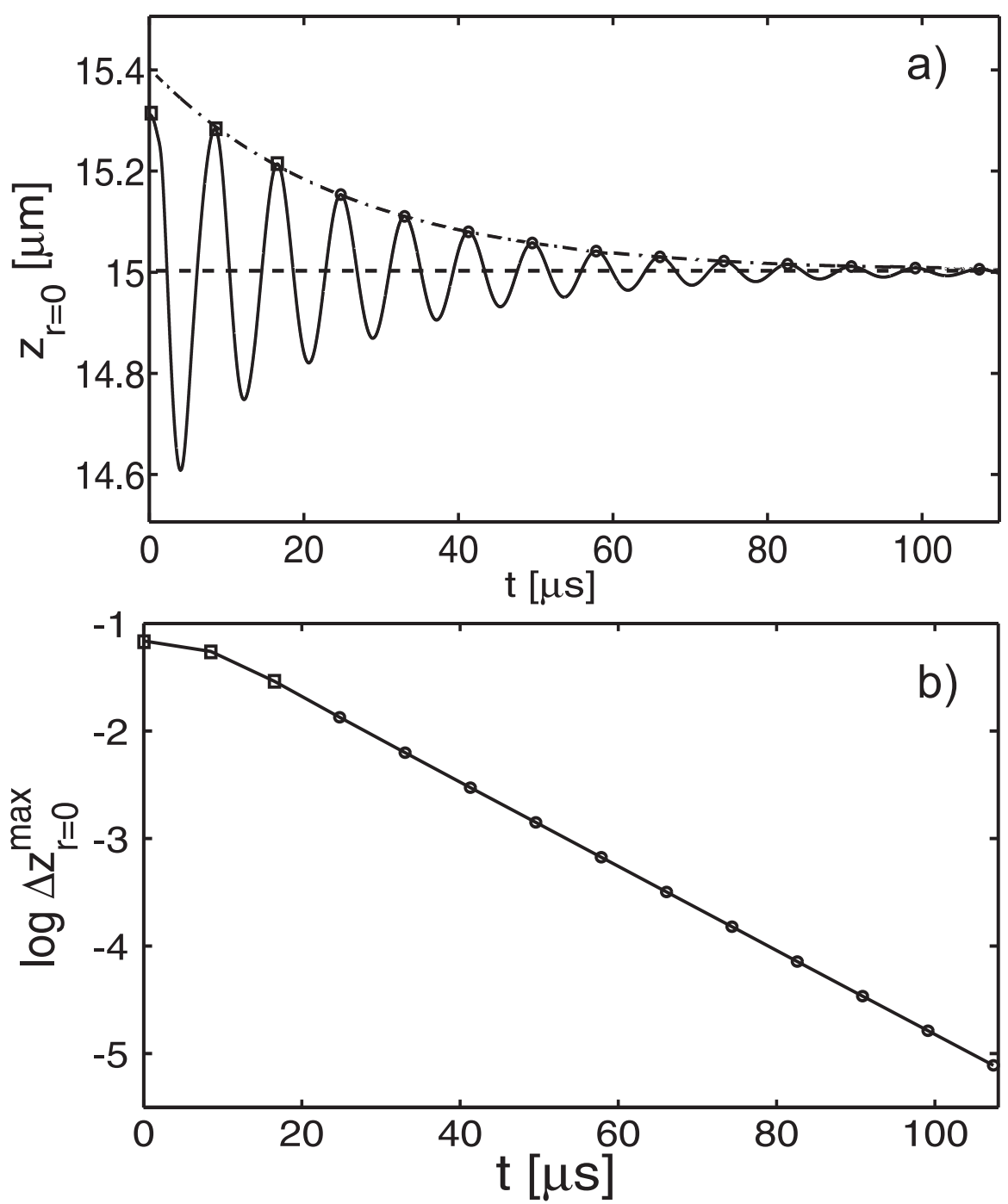

Figure 7.6: Position $z$ of the center of the meniscus (top) and maximum oscillation amplitude $\Delta z$ (bottom) in logarithmic scale as function of time, during free oscillation cycles, in the limiting case of Stokes flow. Parameters: $R_{p}=Z_{p}=15 \mu \mathrm{m}, N_{R}=N_{Z}$ $=250, N_{R p i t}=48$. The dash-dotted line in the top figure is the decaying exponential fitting the oscillation maxima (circles), after the higher modes have decayed. 
we waited for the higher modes to be damped before starting both the calculation of $f_{0}$ and the fit of the exponential $e^{-\beta t}$. In Fig. 7.6.b we show in a logarithmic scale the maxima of the $z(t)$ curve for the Stokes flow. The slope of the straight line connecting two consecutive points gives $\beta$ for that part of the curve. At the beginning, when the higher modes are still present (squares), the apparent damping coefficient is lower, while after some oscillation cycles, when the higher modes are damped out (circles), $\beta$ converges to a higher value, corresponding to the first mode damping coefficient. In Fig. 7.8 and in Fig. 7.9 we present a comparison between the analytical and the numerical result, for different static pressures. For the resonance frequency we found an agreement within the 5\%, while for the damping coefficient we found an agreement within the $10 \%$. The numerical results suggest that convergence is fulfilled, at increasing number of cells in the computational domain (see Fig. 7.10).

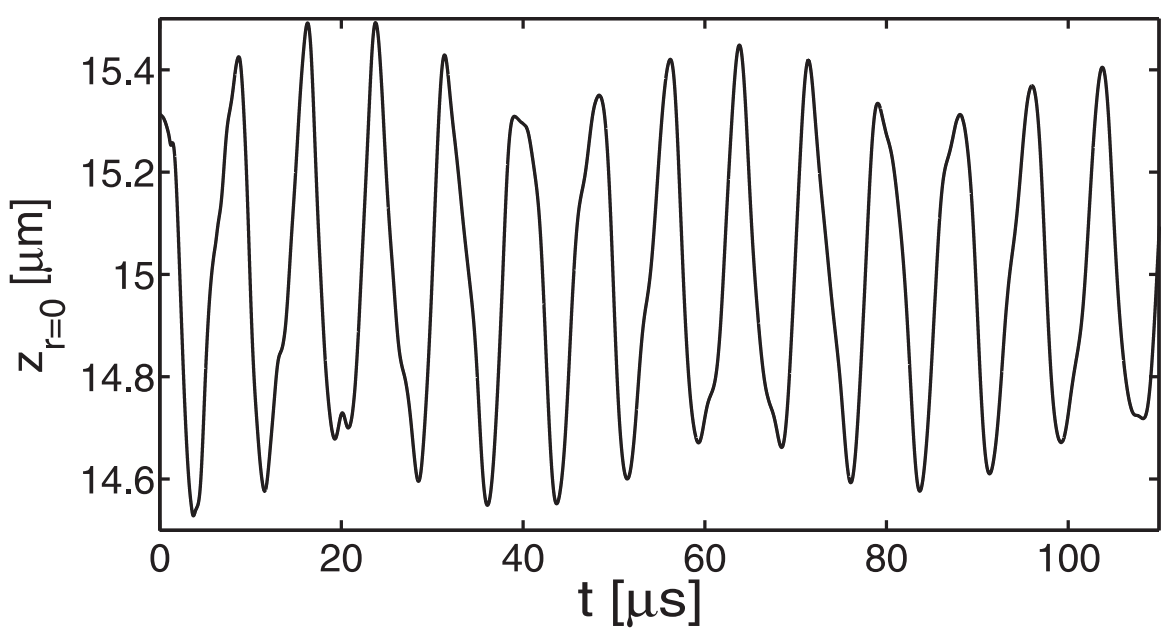

Figure 7.7: Position $z$ of the center of the meniscus as function of time, during free oscillation cycles, in the limiting case of potential flow. Parameters: $R_{p}=Z_{p}=$ $15 \mu \mathrm{m}, N_{R}=N_{Z}=250, N_{\text {Rpit }}=48$. 


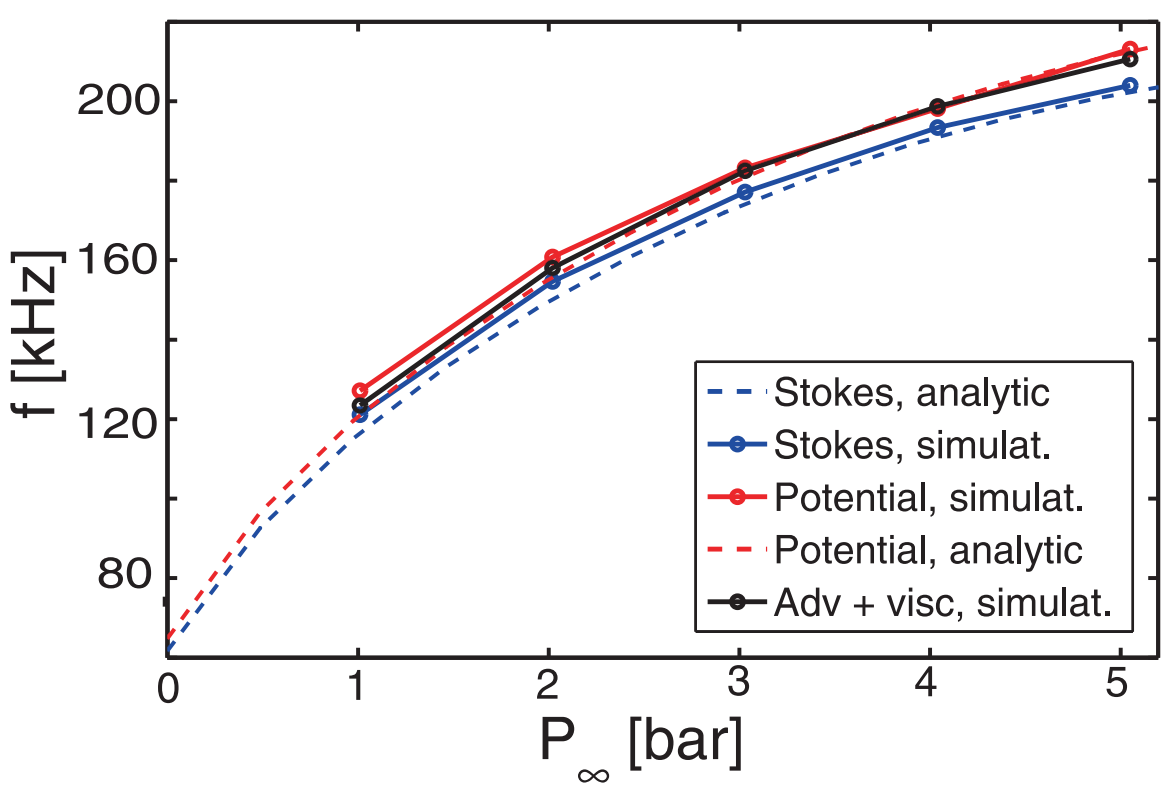

Figure 7.8: Main resonance frequency as function of the static pressure, in the limiting cases of Stokes (blue) and potential flow (red), both numerical (solid) and analytic (dashed). The black line represents the intermediate regime with both advection and viscosity, from simulations. Parameters: $R_{p}=Z_{p}=15 \mu \mathrm{m}, N_{R p i t}=N_{Z p i t}=48$, $N_{R}=N_{Z}=250$.

\subsection{Results}

We numerically investigated the intermediate regime where both advection and viscosity are present. In Fig. 7.8 and Fig. 7.9, we show the resonance frequency and the damping coefficient for different values of the static pressure $P_{\infty}$, in the case of small initial perturbation of the interface and therefore small amplitude oscillations. The resonance frequency always lies between the Stokes and the potential flow resonance frequencies. The damping coefficient is lower when both advection and viscosity are present with respect to the Stokes flow. From both the resonance frequency and the damping coefficient we see that, for lower values of the static pressure, the behavior of the pit is closer to the Stokes flow, while, for higher $P_{\infty}$, it approaches the potential flow behavior.

In order to study the large-amplitude free oscillations, we gave increasing initial deformations to the meniscus, with the shape of a paraboloid 7.19. Though the resonance frequency did not change, the damping decreased when the amplitude of 


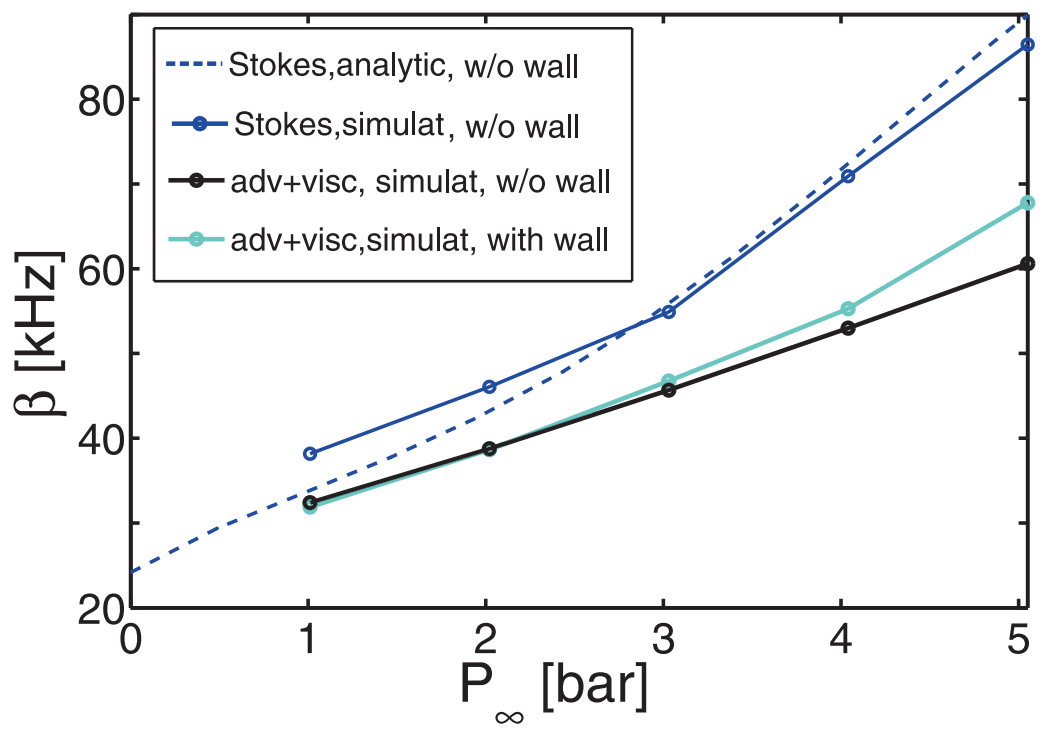

Figure 7.9: Main damping coefficient as function of the static pressure, in the limiting case of Stokes flow without any wall (blue) - analytic (dashed) and from simulations (solid) - and in the regime with both advection and viscosity, with (light blue) and without (black) a wall as right boundary. Parameters: $R_{p}=Z_{p}=15 \mu \mathrm{m}, N_{R}=N_{Z}=$ $250, N_{\text {Rpit }}=48$.

the initial perturbation increased, as shown in Fig. 7.11, where we plot the damping coefficient as a function of the initial displacement $\Delta z_{0}$ of the center of the meniscus.

We also studied the effect of the presence of a rigid boundary both coaxial with the pit (like in the case of a cylindrical container surrounding the pit) or horizontal above it (at the top). This kind of situation arises, for example, when one wishes to use a device such as the one described in Chap. 3 and in Ref. [19] for cleaning purposes $[33,34]$. The presence of a wall increases the damping (see Fig. 7.9) and reduces the resonance frequency (see Fig. 7.12). This effect is a bit more pronounced in the case of the cylindrical wall around the pit with respect to the case of the top boundary wall. The physical phenomena contributing to the reduction of the resonance frequency can be qualitatively explained by considering an analogy with a classical damped harmonic oscillator, undergoing free linear oscillations and its motion equation $\ddot{x}+2 \beta \dot{x}+\omega_{n}^{2} x=0$. Here $x$ is the displacement, $\beta$ is the analogous of the damping coefficient that we are considering for the pit and $\omega_{n}=\sqrt{k / m}$ is the natural frequency of the undamped system, with $k$ the stiffness and $m$ the mass. The 


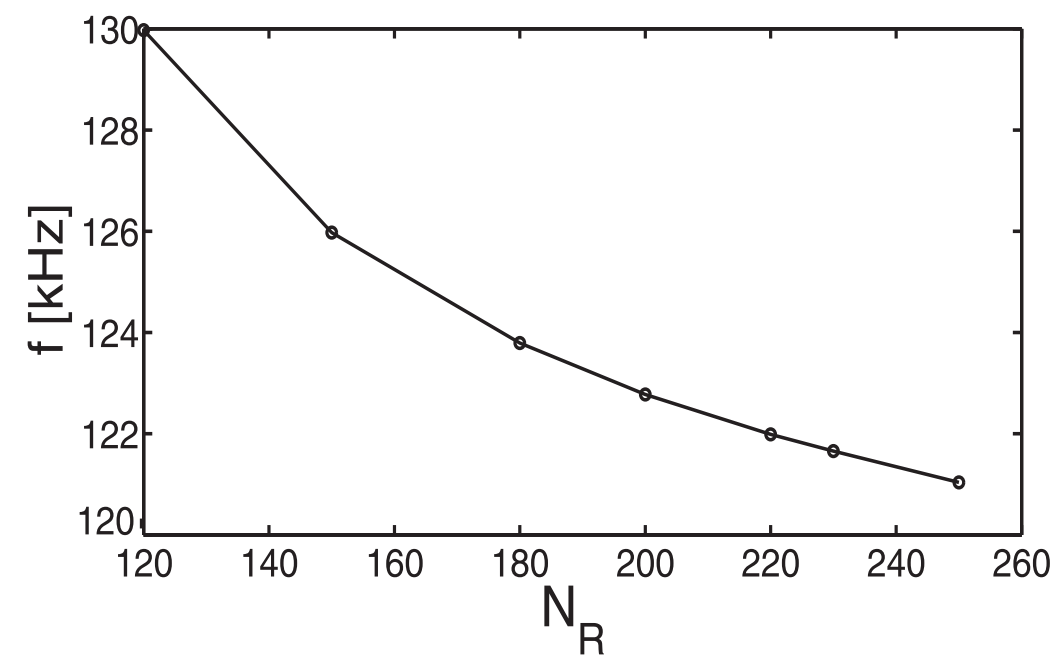

Figure 7.10: Resonance frequency as a function of the number of cells, for the Stokes flow. The analytical value equals $117 \mathrm{kPa}$. The numerical results suggest that convergence is fulfilled. Parameters: $R_{p}=Z_{p}=15 \mu \mathrm{m}, N_{R}=N_{Z}=250, N_{R p i t}=48$.

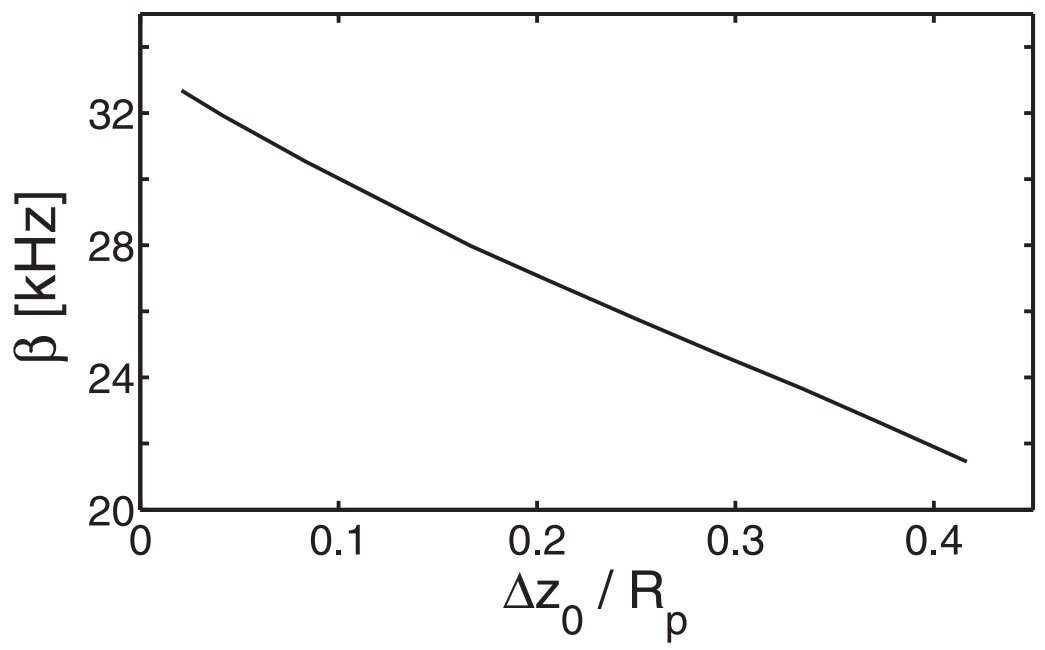

Figure 7.11: Damping coefficient as function of the amplitude of the initial paraboloidal perturbation at the center, in the case with both viscosity and advection, without any wall at the boundaries. Parameters: $R_{p}=Z_{p}=15 \mu \mathrm{m}, N_{R}=N_{Z}=$ $250, N_{\text {Rpit }}=48$. 
eigenfrequency of the damped system is given by $\omega_{0}=\omega_{n} \sqrt{1-\tilde{\beta}^{2}}$, with $\tilde{\beta}=\beta / \omega_{n}$, $\omega_{0}$ the analogous of the resonance frequency that we are considering for the pit and $\omega_{n}$ the undamped resonance frequency, roughly corresponding to the resonance frequency in the potential flow. A reduction of $\omega_{0}$ can be due both to an increase of $\beta$ and to a decrease of $\omega_{n}$. In the case of a pit surrounded be a circular wall, the confinement of the flow changes both the damping coefficient and the natural frequency of the undamped system. In particular, $\beta$ increases because the wall introduces a further nonslip surface, while $\omega_{n}$ decreases, because the added mass rises, leaving unvaried the stiffness, which is given by the surface tension and the elasticity of the gas inside the pit. In order to understand the increase of the added mass due to the confinement, we can think to a pulsating bubble. In the case of an unconfined bubble, the added mass is $m_{A} \sim 4 / 3 \pi R^{3}$, while in the limiting case where the bubble is introduced inside a tube with the same radius, the added mass is $m_{A} \sim \pi R^{2} L$, with $L$ the length of the liquid column.

In other words, when the confinement increases, the propagation of the distur-

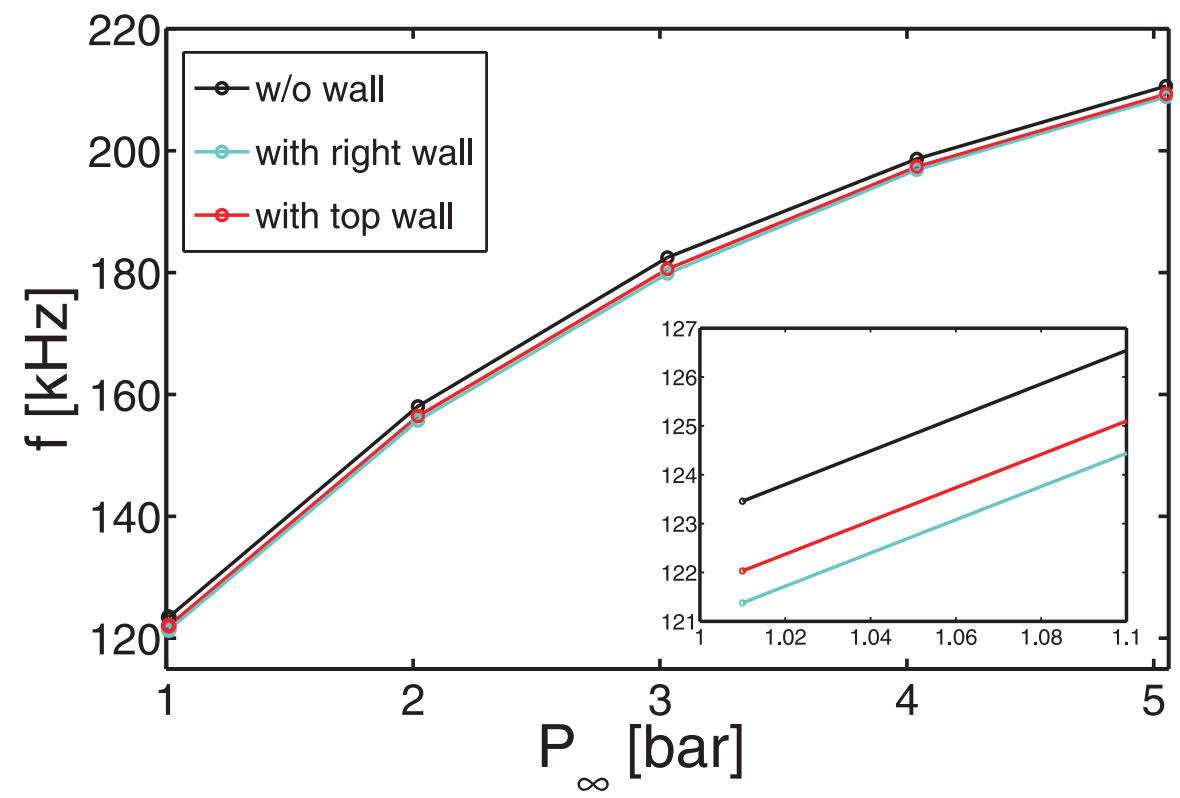

Figure 7.12: Resonance frequency as function of the static pressure, in the case with both viscosity and advection, with a wall as right (light blue) or top (red) boundary and without any wall (black). Parameters: $R_{p}=Z_{p}=15 \mu \mathrm{m}, N_{R}=N_{Z}=250, N_{R p i t}=$ 48. 
bance (and therefore the displaced liquid added mass) are less influenced by the distance respect to the origin of the disturbance itself. Therefore, ideally, if we apply a disturbance to one of the ends of a tube, a flow will be generated that will exit the tube at the other end, independently of the length of the tube itself, with the only limitation given by the propagation speed of the disturbance $c$, in the real case.

In order to investigate the bubble formation mechanism in devices like the sonochemical reactor of Chap. 3 and Ref.[19], we studied the response of the meniscus to a driving pressure. We forced the system through the application of a periodic pressure at the free boundaries, with different frequencies, and we examined the corresponding displacement of the central point of the meniscus, along the $z$-axis. A typical resonance curve is shown in Fig. 7.14. $|\Delta z|$ denotes the maximum oscillation amplitude, after the transient has ended, namely at the $20^{\text {th }}$ cycle (see Fig. 7.13). The maximum response of the meniscus was obtained for the main resonance frequency, as expected. A second lower peak appeared in correspondence of the second resonance frequency (Fig. 7.14).

Given this maximum response in correspondence to the main resonance, we imposed a large sinusoidal initial perturbation and we forced the system at $f_{0}$, to see

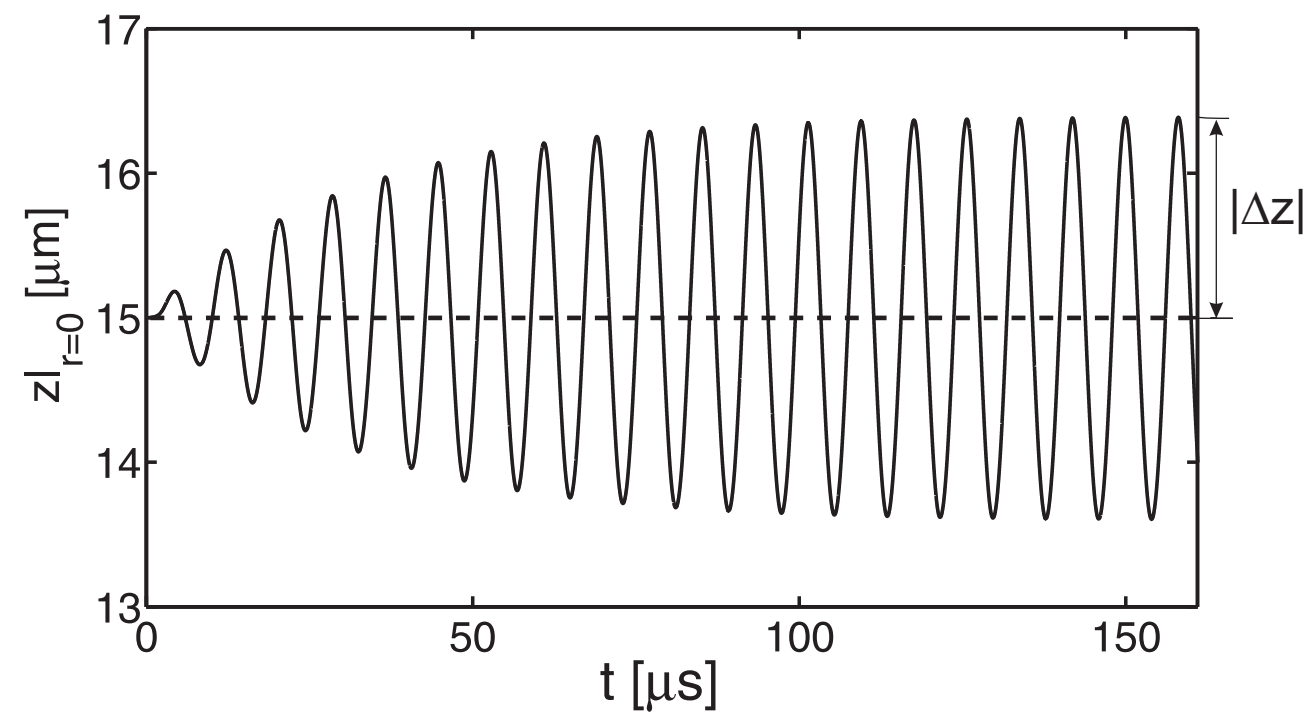

Figure 7.13: Position $z$ of the center of the meniscus as function of time, during forced oscillation cycles, in resonant conditions, when both advection and viscosity are considered. Parameters: $R_{p}=Z_{p}=15 \mu \mathrm{m}, N_{R}=N_{Z}=250, N_{R p i t}=48, f=$ $123.5 \mathrm{kHz}$. 


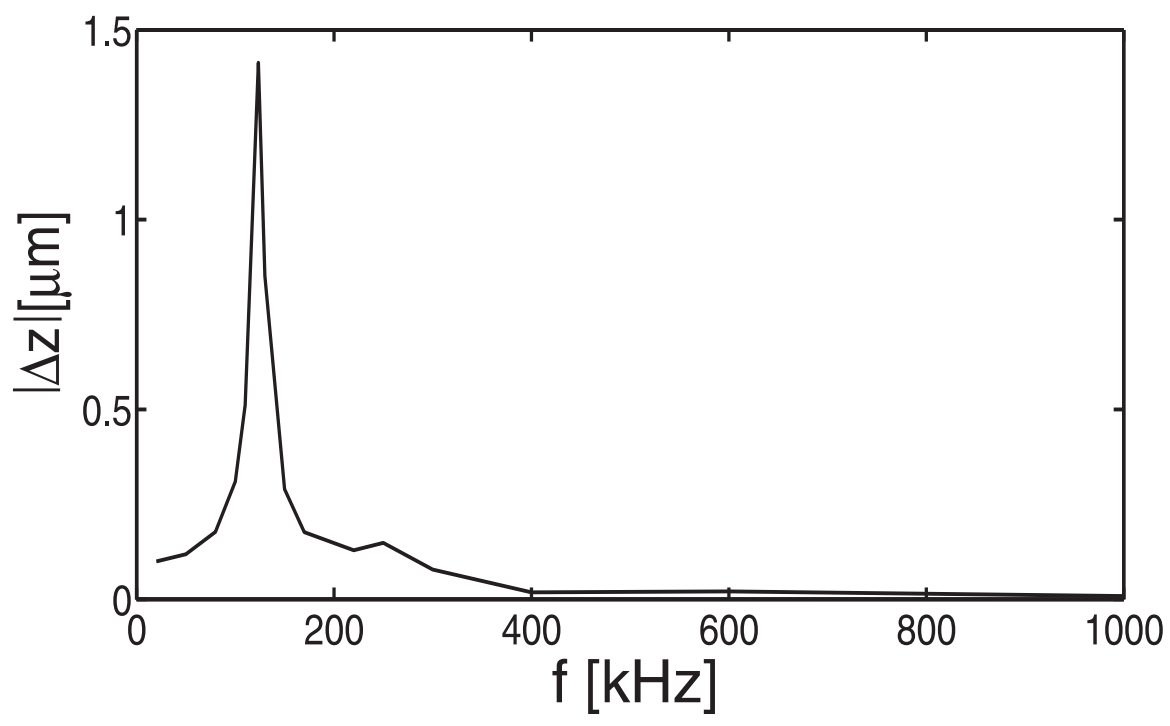

Figure 7.14: Maximum displacement of the central point of the meniscus (at $r=0$ ) undergoing forced oscillations, as function of the driving frequency $f$, during the $20^{t h}$ cycle. Parameters: $R_{p}=Z_{p}=15 \mu \mathrm{m}, P_{a}=1 \mathrm{kPa}, N_{R}=250, N_{R p i t}=48, P_{\infty}$ $=1 \mathrm{~atm}$, free flow both at the right and at the top boundary. The main resonance frequency, as calculated from the free oscillations, is $f_{0}=123.5 \mathrm{kHz}$. A second resonance frequency appears around $250 \mathrm{kHz}$.

if the initial disturbance was amplified, eventually leading to strong instabilities and bubble formation. After a short initial transient of three cycles the initial perturbation relaxed and the meniscus continued to oscillate with a shape close to the first mode (see Fig. 7.15). Therefore we concluded that, in order to asses the bubble formation mechanism, the model should be explored to a larger extent. The bubble formation could be ascribed to a rectified diffusion-like mechanism through the change of the shape of the equilibrium condition or to the excitation of higher modes. These hypothesis will have to be tested in future works. 


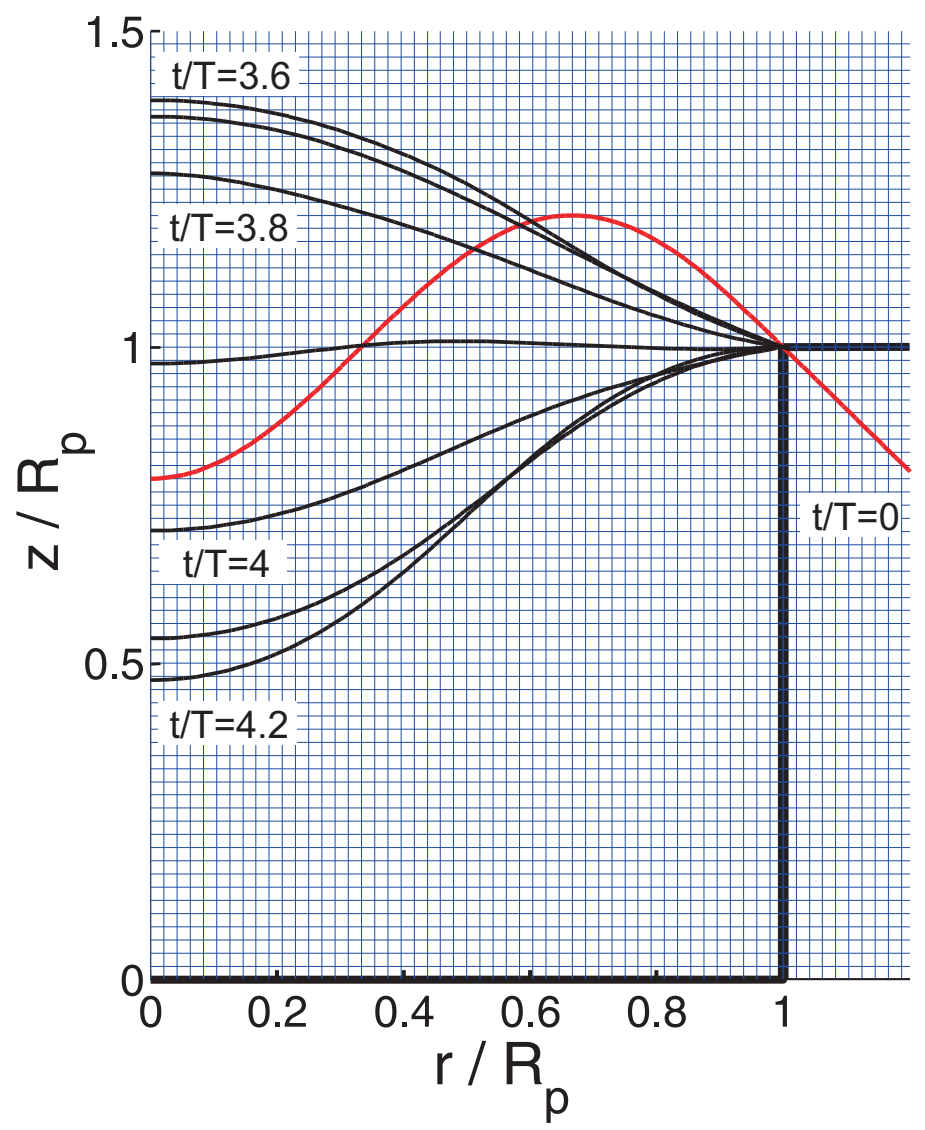

Figure 7.15: Forced oscillation cycle of the meniscus in resonance conditions, with sinusoidal large initial perturbation (red). Parameters: $R_{p}=Z_{p}=15 \mu \mathrm{m}$, $f=123.5 \mathrm{kHz}, P_{a}=6 \mathrm{kPa}$. The initial perturbation line shows also the parabola used to overcome the numerical singularity at the triple point, which is omitted in the other instants for clarity reasons. The black lines correspond to the interface position at instants $t_{i+1}=t_{i}+d t$, with $d t=0.1 T$ and $T=1 / f$.

\subsection{Summary}

The aim of the present work was the studying of the dynamics of an entrapped bubble inside a cylindrical micropit etched over a planar substrate, like those of the sonochemical reactor described in Chap. 3 and in Ref. [19]. We neglected mass and heat diffusion and we imposing the no-slip condition at the contact line. We used a numerical approach based on the level set method for the interface tracking, together with 
a projection method for the solution of the Navier-Stokes equations in the liquid. In order to validate the code, we compared the main resonance frequency and damping coefficient from simulations with the analytical ones [24] in the limiting cases of small amplitude oscillations in Stokes and potential flow. We found an agreement within the $5 \%$ and the $10 \%$ for the resonance frequency and the damping coefficient, respectively. We examined the intermediate regime where both viscosity and advection were present and we found that the resonance frequency lay in between the two limiting cases, with a shift from Stokes-like to potential-like values, as the static pressure increased. The damping coefficient was always lower with respect to the one of the Stokes flow. From the study of the large amplitude free oscillations, we found that the resonance frequency did not change when the initial perturbation was increased, while the damping coefficient decreased. We examined the effect of the presence of a wall, situation that could occur in cleaning devices such as those of Refs. [33, 34]. The introduction of a rigid wall either around or above the pit, caused a decrease of the resonance frequency and an increase of the damping coefficient. We examined the effects of forced oscillations upon the pit, finding resonance curves in agreement with the values calculated from the free oscillations, as a higher response amplitude was found in resonance conditions. Secondary resonance peaks also appeared. Despite the large response of the gas pocket in resonant condition, we verified that, even for large initial perturbations, the forced oscillations tended to smoothen the surface instabilities, thus leading to a motion similar to the one of the first oscillating mode. Therefore we could conclude that the mechanism of bubble formation found in sonochemical reactors of the kind of Chap. 3 and Ref. [19] cannot be ascribed to the excitation of the main resonance frequency, when the meniscus has a flat equilibrium condition. Possible underlying mechanisms could be a rectified diffusion-like mechanism, through the deformation of the equilibrium condition of the interface that it induces, or the excitation of higher modes. Both these hypothesis will have to be verified in future works.

\subsection{Appendix A}

In order to validate the pressure field from the solution of the Poisson equation (7.18) we consider a test case with a flat meniscus, with zero-velocity and a rigid wall as right boundary. We compare the numerical results with the analytical solution of the mixed boundary problem for the Laplace's equation in cylindrical geometry:

$$
\nabla^{2} p=0,
$$


subject to the boundary conditions (see Fig. 7.3)

$$
\begin{aligned}
\frac{\partial p}{\partial r}(r, 0) & =\frac{\partial p}{\partial r}(r, R)=0 & & \\
p(r, H) & =p_{\infty} & & 0 \leq r<R_{p} \\
p(z=0) & =p_{\Gamma}, & & R_{p}<r \leq R
\end{aligned}
$$

Given the cylindrical geometry, we first look for the solution in terms of a Fourier series, we substitute it inside (7.20) and through (7.21), we rewrite it in terms of the Fourier-Bessel series [44]

$$
p(r, z)=\sum_{k=1}^{\infty} Z_{k}(z) J_{0}\left(j_{k}^{(1)} \frac{r}{R}\right),
$$

where $J_{0}$ is the first type Bessel function of order 0 and $j_{k}^{(1)}$ are the zero's of the first type Bessel function of order 1, $J_{1}$. Upon substitution inside (7.20), we get

$$
\frac{d^{2} Z_{k}}{d z^{2}}-Z_{k}\left(\frac{j_{k}^{(1)}}{R}\right)^{2}=0,
$$

from which

$$
p(r, z)=D_{0}+C_{0} z+\sum_{k=1}^{\infty}\left[A_{k} \sinh \frac{j_{k}^{(1)}(H-z)}{R}+B_{k} \cosh \frac{j_{k}^{(1)}(H-z)}{R}\right] J_{0}\left(j_{k}^{(1)} \frac{r}{R}\right) .
$$

Therefore the problem reduces to finding the constant coefficients $A_{k}, B_{k}, C_{0}$ and $D_{0}$. From the boundary condition (7.22), performing the projection operation, we get

$$
\sum_{k=1}^{\infty} B_{k} \int_{0}^{R} r J_{0}^{2}\left(j_{k}^{(1)} \frac{r}{R}\right) d r=p_{\infty} \int_{0}^{R} r J_{0}\left(j_{k}^{(1)} \frac{r}{R}\right) d r
$$

Here the standard orthogonality property of the Bessel functions cannot be used, because in the argument of the $J_{0}$ there are not the zero's of $J_{0}, j_{k}$, but there are the $j_{k}^{(1)}$, at the left hand side of (7.28). Therefore a preliminary manipulation is required. By making use of the properties of the Bessel functions [45] $\int_{0}^{a} J_{n}^{2}(\lambda r) r d r=$ $\frac{a^{2}}{2}\left[J_{n}^{\prime 2}(\lambda a)+\left(a-\frac{n^{2}}{\lambda^{2} a^{2}}\right) J_{n}^{2}(\lambda a)\right]$ where $\lambda$ and $a$ are constants, $n$ is the order of the 
Bessel function, and the apostrophe denotes the derivative respect to $r$, and with the further use of and $J_{0}^{\prime}\left(j_{k}^{(1)}\right)=-J_{1}\left(j_{k}^{(1)}\right)=0$, we get

$$
B_{k}=\frac{2 p_{\infty}}{R^{2} J_{0}^{2}\left(j_{k}^{(1)}\right)} \int_{0}^{R} r J_{0}\left(j_{k}^{(1)} \frac{r}{R}\right) d r
$$

By making use of the property $x J_{0}(x)=x J_{1}^{\prime}(x)+J_{1}(x)$, the integral at the right hand side of (7.29) can be rewritten as

$$
\begin{aligned}
\int_{0}^{R} r J_{0}\left(j_{k}^{(1)} \frac{r}{R}\right) d r & =\frac{R}{j_{k}^{(1)}} \int_{0}^{R} j_{k}^{(1)} \frac{r}{R} J_{1}^{\prime}\left(j_{k}^{(1)} \frac{r}{R}\right)+J_{1}\left(j_{k}^{(1)} \frac{r}{R}\right) d r \\
& =\frac{R}{j_{k}^{(1)}} \int_{0}^{R} \frac{d}{d r}\left[r J_{1}\left(j_{k}^{(1)} \frac{r}{R}\right)\right] \\
& =0 .
\end{aligned}
$$

Therefore, $B_{k}=0$, from which $C_{0}=\left(p_{\infty}-D_{0}\right) / H$. Upon substitution inside (7.27), we find

$$
p(r, z)=\sum_{k=1}^{\infty} A_{k} \sinh \frac{j_{k}^{(1)}(H-z)}{R} J_{0}\left(j_{k}^{(1)} \frac{r}{R}\right)+\frac{p_{\infty}-D_{0}}{H} z+D_{0}
$$

In order to calculate the $A_{k}$ and $D_{0}$ coefficients, we use the remaining boundary condition at $z=0$. Upon substitution of (7.23),(7.24) inside (7.31), the problem can be reformulated in terms of the following dual series formulation

$$
\begin{array}{rlrl}
\sum_{k=1}^{\infty} A_{k} \sinh \frac{j_{k}^{(1)} H}{R} J_{0}\left(j_{k}^{(1)} \frac{r}{R}\right)+D_{0}=p_{\Gamma}, & & 0 \leq r<R_{p} \\
\sum_{k=1}^{\infty} A_{k} \cosh \frac{j_{k}^{(1)} H}{R} J_{0}\left(j_{k}^{(1)} \frac{r}{R}\right)+\frac{p_{\infty}-D_{0}}{H}=0, & R_{p}<r \leq R
\end{array}
$$

This problem cannot be directly solved by means of the classical projection operation, because the orthogonality property of $J_{0}\left(j_{k}^{(1)} r / R\right)$ holds on the interval $0 \leq r \leq R$, while (7.32)(7.33) are valid only on a part of it. Therefore, we need to find an expression for one of the two boundary conditions, valid on the whole domain. We make the hypothesis that $\left.\frac{\partial p}{\partial z}\right|_{z=0}$ has the shape

$$
\left.\frac{\partial p}{\partial z}\right|_{z=0}=\frac{q(r)}{\sqrt{R_{p}^{2}-r^{2}}} H\left(R_{p}-r\right), \quad 0 \leq r \leq R
$$


where $H$ is the Heaviside function and

$$
q(r)=\sum_{n=1}^{\infty} q_{n} T_{2(n-1)}\left(r / R_{p}\right)
$$

in which $q_{n}$ are constants to be determined and $T_{2(n-1)}$ are the even Chebyshev polynomials, taken such to satisfy the symmetry requirement respect to the $z$ axes. Upon substitution of (7.34),(7.35) inside (7.33), we get

$$
\sum_{k=1}^{\infty} A_{k} \cosh \frac{j_{k}^{(1)} H}{R} J_{0}\left(j_{k}^{(1)} \frac{r}{R}\right)+\frac{p_{\infty}-D_{0}}{H}=\frac{\sum_{n=1}^{\infty} q_{n} T_{2(n-1)}\left(r / R_{p}\right)}{\sqrt{R_{p}^{2}-r^{2}}} H\left(R_{p}-r\right),
$$

valid on $0 \leq r \leq R$. By performing the projection operation and using orthogonality, after some algebra we find

$$
\begin{gathered}
D_{0}=p_{\infty}-\frac{2 H}{R^{2}} \sum_{n} q_{n} b_{n}, \\
A_{k}=p\left(j_{k}^{(1)}\right) \sum_{n} q_{n} a_{n}\left(j_{k}^{(1)}\right),
\end{gathered}
$$

with

$$
\begin{aligned}
b_{n} & =\int_{0}^{R_{p}} \frac{r T_{2(n-1)}\left(r / R_{p}\right)}{\sqrt{R_{p}^{2}-r^{2}}} d r, \\
a_{n}\left(j_{k}^{(1)}\right) & =\int_{0}^{R_{p}} \frac{r T_{2(n-1)}\left(r / R_{p}\right) J_{0}\left(j_{k}^{(1)} r / R\right)}{\sqrt{R_{p}^{2}-r^{2}}} d r, \\
p\left(j_{k}^{(1)}\right) & =-\frac{R}{j_{k}^{(1)} \cosh \left(j_{k}^{(1)} \frac{H}{R}\right) N_{0, k}}, \\
N_{0, k} & =\int_{0}^{R} J_{0}^{2}\left(j_{k}^{(1)} \frac{r}{R}\right) r d r .
\end{aligned}
$$


After some further algebra

$$
\begin{aligned}
& b_{n}=\frac{R_{p} \sin [(2 n-3) \pi / 2]}{(2 n-1)(2 n-3)}, \\
& a_{n}\left(j_{k}^{(1)}\right)=\frac{\pi R_{p}}{4}\left[J_{n-\frac{1}{2}}\left(\frac{j_{k}^{(1)} R_{p}}{2 R}\right) J_{\frac{1}{2}-n}\left(\frac{j_{k}^{(1)} R_{p}}{2 R}\right)+\right. \\
& \left.J_{n-\frac{3}{2}}\left(\frac{j_{k}^{(1)} R_{p}}{2 R}\right) J_{\frac{3}{2}-n}\left(\frac{j_{k}^{(1)} R_{p}}{2 R}\right)\right], \\
& p\left(j_{k}^{(1)}\right)=-\frac{1}{2}\left[R j_{k}^{(1)} \cosh \frac{j_{k}^{(1)} H}{R} J_{0}^{2}\left(j_{k}^{(1)}\right)\right]^{-1} .
\end{aligned}
$$

Upon substitution of (7.38) and (7.37) inside (7.32), the following expression is found:

$$
p_{\infty}-\frac{2 H}{R^{2}} \sum_{n} q_{n} b_{n}+\sum_{k} p\left(j_{k}^{(1)}\right) \sinh \frac{j_{k}^{(1)}(H-z)}{R} J_{0}\left(j_{k}^{(1)} \frac{r}{R}\right) \sum_{n} q_{n} a_{n}\left(j_{k}^{(1)}\right)=p_{\Gamma} .
$$

By inverting $\sum_{n}$ and $\sum_{k}$ and performing the projection operation $\int_{0}^{R_{p}}(\cdot) T_{2(m-1)}\left(r / R_{p}\right) /\left(R_{p}^{2}-\right.$ $\left.r^{2}\right)^{1 / 2} d r$, after some algebra we find

$$
\sum_{n} q_{n} K_{m n}=c_{m}, \quad(m=1, \ldots, \infty)
$$

where

$$
\begin{aligned}
K_{m n} & =-\frac{2 H}{R^{2}} b_{n} t_{m}+\sum_{k} r\left(j_{k}^{(1)}\right) a_{n}\left(j_{k}^{(1)}\right) S_{m}\left(j_{k}^{(1)}\right), \\
c_{m} & =\left(p_{\Gamma}-p_{\infty}\right) t_{m}
\end{aligned}
$$


in which

$$
\begin{aligned}
r\left(j_{k}^{(1)}\right) & =\sinh \frac{j_{k}^{(1)}(H-z)}{R} p\left(j_{k}^{(1)}\right), \\
t_{m} & =\int_{0}^{R_{p}} \frac{T_{2(m-1)}\left(r / R_{p}\right)}{\sqrt{R_{p}^{2}-r^{2}}} d r, \\
S_{m}\left(j_{k}^{(1)}\right) & =\int_{0}^{R_{p}} \frac{J_{0}\left(j_{k}^{(1)} r / R\right) T_{2(m-1)}\left(r / R_{p}\right)}{\sqrt{R_{p}^{2}-r^{2}}} d r .
\end{aligned}
$$

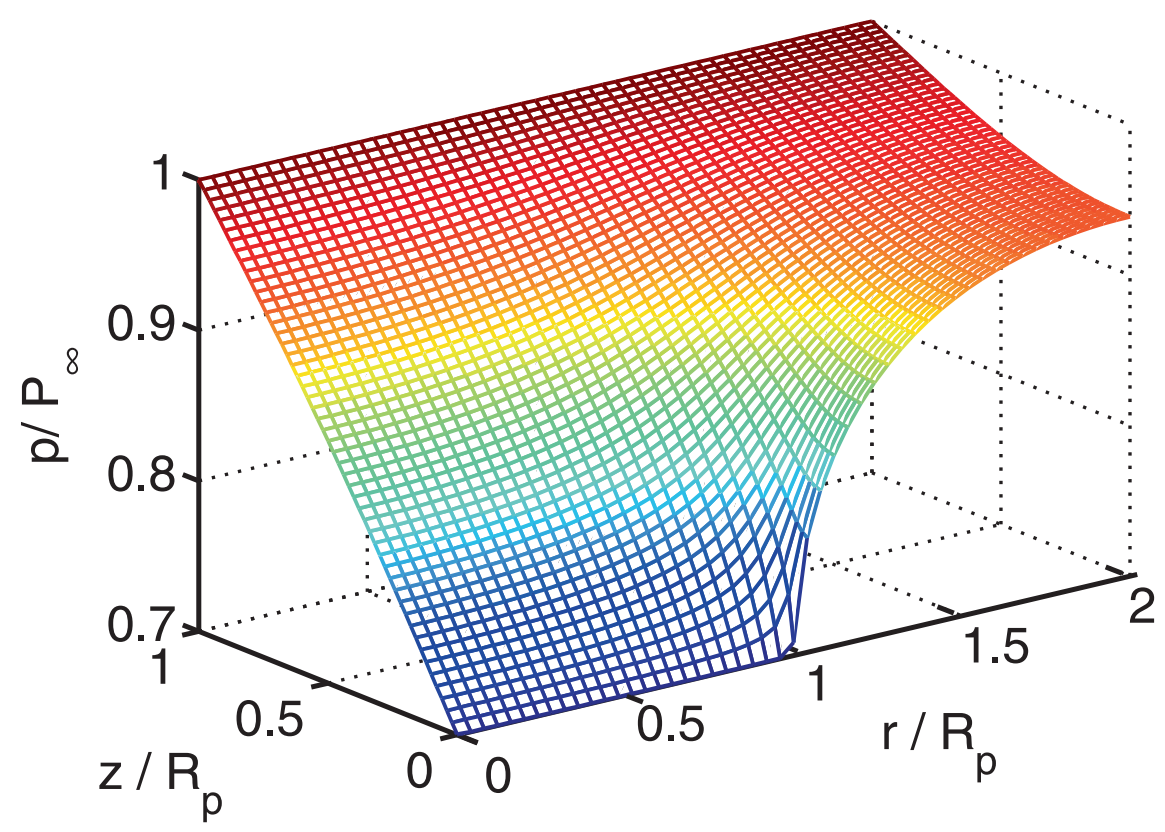

Figure 7.16: Analytical solution of the mixed boundary Laplace problem used as test case for the pressure field in Sec.7.4. Parameters: $R_{p}=R / 2=Z, p_{\Gamma}=0.7 P_{\infty}$. The pit here corresponds to the negative values of $z$. 
After some algebra

$$
\begin{aligned}
r\left(j_{k}^{(1)}\right) & =-\frac{2}{R j_{k}^{(1)} J_{0}^{2}\left(j_{k}^{(1)}\right)} \tanh \frac{j_{k}^{(1)} H}{R}, \\
t_{m} & =\frac{\pi}{2} \delta_{m 1}, \\
S_{m}\left(j_{k}^{(1)}\right) & =\frac{\pi}{2} J_{m-1}\left(\frac{j_{k}^{(1)} R_{p}}{2 R}\right) J_{1-m}\left(\frac{j_{k}^{(1)} R_{p}}{2 R}\right) .
\end{aligned}
$$

By truncating the infinite series of 7.35 to a finite number of terms $N$, chosen such to match convergence criteria, the following algebraic system is retrieved

$$
\left[K_{m n}\right]\left\{q_{n}\right\}=\left\{c_{m}\right\},
$$

where $\left[K_{m n}\right]$ is a $N x N$ matrix and $\left\{q_{n}\right\}$ is the vector of the $N$ unknowns, $q_{n}$. In order to calculate the terms $K_{m n}$ we truncate the infinite series of (7.50) to a finite number of terms $M$, chosen such to have convergence for the each $K_{m n}$. We calculate the $q_{n}$ by solving the system (7.58) and we substitute them inside (7.38) and (7.37) to get the coefficients $A_{k}, D_{0}$ which, upon substitution inside (7.31), eventually give the $p(r, z)$. For practical reasons, (7.31) is truncated to a finite number of terms, $N_{k}$. We have verified that convergence requirements are fulfilled for $N_{k}=200, N=4, M=10$.

For numerical implementation, it is convenient to rewrite (7.31) in such a way that it does not diverge for large $j_{k}^{(1)}$

$p(r, z)=\sum_{k=1}^{N_{k}} E_{k} \exp \left(-\frac{j_{k}^{(1)} z}{R}\right) J_{0}\left(j_{k}^{(1)} \frac{r}{R}\right)\left[1-\exp \left(-2 j_{k}^{(1)} \frac{H-z}{R}\right)\right]+\frac{p_{\infty}-D_{0}}{H} z+D_{0}$,

in which

$$
E_{k}=-\frac{2 \sum_{n=1}^{N} q_{n} a_{n}\left(j_{k}^{(1)}\right)}{j_{k}^{(1)} R J_{0}^{2}\left(j_{k}^{(1)}\right)\left[1+\exp \left(-2 j_{k}^{(1)} \frac{H}{R}\right)\right]} .
$$

In Fig. 7.16 we show a 3D view of the pressure field. 


\section{References}

[1] R. E. Apfel, "The role of impurities in cavitation-threshold determination", J. Acoust. Soc. Am. 48, 1179-1189 (1970).

[2] N. Bremond, M. Arora, C. D. Ohl, and D. Lohse, "Controlled multibubble surface cavitation", Phys. Rev. Lett. 96, 224501 (2006).

[3] N. Bremond, M. Arora, S. M. Dammer, and D. Lohse, "Interaction of cavitation bubbles on a wall", Phys. Fluids 18, 121505 (2006).

[4] W. L. Nyborg and D. L. Miller, "Biophysical implications of bubble dynamics", Appl. Sci. Research 38, 17-24 (1982).

[5] W. L. Nyborg, "Ultrasonic microstreaming and related phenomena", Br. J. Cancer 45, Suppl. 5, 156 (1982).

[6] D. L. Miller, "Particle gathering and microstreaming near ultrasonically activated gas-filled micropores", J. Acoust. Soc. Am. 84, 1378-1387 (1988).

[7] R. H. Liu, J. N. Yang, M. Z. Pindera, M. Athavale, and P. Grodzinski, "Bubbleinduced acoustic micromixing", Lab on a Chip 2, 151-157 (2002).

[8] D. L. Miller, "Cell death thresholds in Elodea for 0.45-10 MHz ultrasound compared to gas-body resonance theory", Ultras. Med. Biol. 5, 351-357 (1979).

[9] S. Z. Child, E. L. Carstensen, and S. K. Lam, "Effects of ultrasound on drosophila: III. Exposure of larvae to low-temporal-average-intensity, pulsed irradation", Acoust. Med. Biol. 7, 167-173 (1981).

[10] D. L. Miller, W. Nyborg, and C. Whitcomb, "Platelet aggregation induced by ultrasound under specialized conditions in vitro", Science 205, 505-507 (1979).

[11] A. R. Williams and D. L. Miller, "Photometric detection of ATP release from human erythrocytes exposed to ultrasonically activated gas-filled pores", Ultras. Med. Biol. 6, 251-256 (1980).

[12] D. L. Miller, "Frequency relationships for ultrasonic activation of free microbubbles, encapsulated microbubbles, and gas-filled micropores", J. Acoust. Soc. Am. 104, 2498-2505 (1998).

[13] L. A. Kuznetsova, S. Khanna, N. N. Amso, W. T. Coakley, and A. A. Doinikov, "Cavitation bubble-driven cell and particle behavior in an ultrasound standing wave", J. Acoust. Soc. Am. 117, 104-112 (2005). 
[14] M. Postema and O. H. Gilja, "Ultrasound-directed drug delivery", Curr. Pharm. Biotechnol. 8, 355-361 (2007).

[15] R. J. Browning, H. Mulvana, M.-X. Tang, J. V. Hajnal, D. J. Wells, and R. J. Eckersley, "Effect of albumin and dextrose concentration on ultrasound and microbubble mediated gene transfection in vivo", Ultrasound Med. Biol. 38, 1067-1077 (2012).

[16] D. L. Miller, "Experimental investigation of the response of gas-filled micropores to ultrasound", J. Acoust. Soc. Am. 71, 471-476 (1982).

[17] B. Borkent, S. Gekle, A. Prosperetti, and D. Lohse, "Nucleation threshold and deactivation mechanisms of nanoscopic cavitation nuclei", Phys. Fluids 102003 (2009).

[18] D. Fernández Rivas, A. Prosperetti, A. G. Zijlstra, D. Lohse, and H. J. G. E. Gardeniers, "Efficient sonochemistry through microbubbles generated with micromachined surfaces", Angew. Chem. Int. Ed. 49, 9699-9701 (2010).

[19] D. Fernández Rivas, L. Stricker, A. Zijlstra, H. Gardeniers, D. Lohse, and A. Prosperetti, "Ultrasound artificially nucleated bubbles and their sonochemical radical production", Ultrason. Sonochem. 20, 510-524 (2013).

[20] E. A. Neppiras, W. L. Nyborg, and D. L. Miller, "Non-linear behaviour and stability of trapped micron-sized cylindrical gas bubbles in an ultrasonic field", Ultrasonics 21, 109-115 (1983).

[21] H. Rathgen, K. Sugiyama, C.-D. Ohl, D. Lohse, and F. Mugele, "Nanometerresolved collective micromeniscus oscillations through optical diffraction", Phys. Rev. Lett. 99, 214501 (2007).

[22] K. Paumel, J. Moysan, M. Autric, C. Gueudré, G. Corneloup, and F. Baqué, "Visualization of the ultrasound-induced behaviour of gas pockets entrapped on a patterned surface. application to inspection of sodium-cooled fast reactors", Nucl. Eng. Des. 239, 2272-2278 (2009).

[23] D. L. Miller and W. L. Nyborg, "Theoretical investigation of the response of gas-filled micropores and cavitation nuclei to ultrasound", J. Acoust. Soc. Am. 73, 1537-1544 (1983).

[24] H. Gelderblom, A. G. Zijlstra, L. van Wijngaarden, and A. Prosperetti, "Oscillations of a gas pocket on a liquid-covered solid surface", Phys. Fluids 24, 122101 (2012). 
[25] E. Can, "Vapor bubbles in confined geometries: a numerical study", Ph.D. thesis, University of Twente (2010).

[26] E. Can and A. Prosperetti, "A level set method for vapor bubble dynamics", J. Comp. Phys. 231, 1533-1552 (2012).

[27] S. Osher and R. Fedkiw, Level set methods and dynamic implicit surfaces (Springer, New York) (2003).

[28] J. A. Sethian, Level set methods and fast marching methods (Cambridge University Press, Cambridge) (1999).

[29] J. A. Sethian and P. Smereka, "Level set methods for fluid interfaces", Annu. Rev. Fluid Mech. 35, 341-372 (2003).

[30] J. A. Sethian and J. Strain, "Crystal growth and dendritic solidification", J. Comp. Phys. 98, 231-253 (1992).

[31] F. Gibou, L. Chen, D. Nguyen, and S. Banerjee, "A level set based sharp interface method for the multiphase incompressible Navier-Stokes equations with phase change", J. Comp. Phys. 222, 536-555 (2007).

[32] J.-D. Yu, S. Sakai, and J. A. Sethian, “Two-phase viscoelastic jetting”, J. Comp. Phys. 220, 568-585 (2007).

[33] D. Fernández Rivas, “Taming acoustic cavitation”, Ph.D. thesis, University of Twente (2012).

[34] D. Fernández Rivas, B. Verhaagen, J. R. T. Seddon, A. G. Zijlstra, L.-M. Jiang, L. W. M. van der Sluis, M. Versluis, D. Lohse, and H. J. G. E. Gardeniers, "Localized removal of layers of metal, polymer, or biomaterial by ultrasound cavitation bubbles", Biomicrofluidics 6, 034114 (2012).

[35] M. J. Choi, A. J. Coleman, and J. E. Saunders, “The influence of fluid properties and pulse amplitude on bubble dynamics in the field of a shock wave lithotripter", Phys. Med. Biol. 38, 1561-1573 (1993).

[36] M. Sussman, P. Smereka, and S. Osher, "A level set approach for computing solutions to incompressible two-phase flow”, J. Comput. Phys. 146-159 (1994).

[37] J. A. Sethian, "A fast marching level set method for monotonically advancing fronts”, Proc. Nat. Acad. Sci. 1591-1595 (1996). 
[38] G. S. Jiang and D. Peng, "Weighted ENO schemes for Hamilton Jacobi equations”, SIAM J. Sci. Comput. 21, 2126-2143 (2000).

[39] C. W. Shu and S. Osher, "Efficient implementation of essentially non oscillatory shock capturing schemes", J. Comput. Phys. 439-471 (1988).

[40] a. P. S. G. Russo, "A remark on computing distance functions", J. Comp. Phys. 163, 51-67 (2000).

[41] A. J. Chorin, "Numerical solution of the Navier-Stokes equations", Math. Comput. 22, 745-762 (1968).

[42] M. Sussman, "A second order coupled level set and volume-of-fluid method for computing growth and collapse of vapor bubbles", J. Comput. Phys. 187, 110-136 (2003).

[43] G. K. Batchelor, An Introduction to Fluid Dynamics (Cambridge University Press, Cambridge) (1970).

[44] A. Prosperetti, Advanced mathematics for applications (Cambridge Univ. Press, Cambridge) (2011).

[45] A. Grey and G. B. Mathews, A treatise on Bessel functions and their applications to physics (Macmillan \& co., London) (1895). 


\section{8 \\ Interacting bubble clouds and their sonochemical production $* \dagger$}

In the present work we study the interaction between bubble clusters generated from acoustically driven air pockets trapped upon a surface. In particular, we examine the transition between the three different behaviors observed in the new generation sonochemical reactors of Chap. 3, at increasing powers: clusters close to the very pits out of which they had been created, clusters pointing toward each other and merging clusters. The latter behavior is highly undesired for technological purposes as it is associated to a reduction of the radical production and an enhancement of the erosion of the reactor walls. We study the conditions required for the transition to occur and its dependence on several parameters such as the distance of the pits. We also show that the underlying mechanism, governed by the secondary Bjerknes forces, is strongly influenced by the nonlinearity of the bubble oscillations but does not depend on the number of nucleated bubbles. We show that the Bjerknes forces dampen the bubble oscillations, thus reducing the radical production and that the increased number of bubble at high power could be the key to understand the experimental observation that, after a certain power threshold, any further increase of the driving does not improve the sonochemical efficiency but can eventually reduce it.

\footnotetext{
*Submitted to J. Acoust. Soc. Am. as: [Laura Stricker, Benjamin Dollet, David Fernández-Rivas and Detlef Lohse, Interacting bubble clouds and their sonochemical production ].

${ }^{\dagger}$ The experimental data present in this chapter are entirely due to David Fernández-Rivas.
} 


\subsection{Introduction}

Fernández-Rivas et al. [1, 2] have recently proposed an efficient way to do sonochemistry, controlling cavitation by using micropits grooved on silicon substrates, following the original idea of Bremond et al. [3]. At sufficient acoustic pressures, a bubble cluster is generated in the liquid above each pit. If two pits or more are present, such clusters tend to attract and merge over a certain pressure amplitude. It has been shown that merging clusters are associated to a reduction in the radical production respect to the case of not interacting clusters [2] and erosion of the reactor walls [4]. Therefore, in efficient sonochemical reactors design, one should maximize the number of pits (i.e. of bubbles) but should avoid cluster-merging conditions. The goal of the present work is to understand the transition between the three possible behaviors observed in experiments (see Fig. 8.1): individual clusters next to the pit out of which they were generated (behavior 1), individual clusters pointing towards the central array of the pits (behavior 2), and clusters migrating towards the center (behavior 3). The key factor to study these phenomena are the acoustic interactions between different bubbles, namely the secondary Bjerknes forces $[5,6]$.

Though these forces have been largely investigated both for bubble pairs [7-12] and for bubble clouds $[13,14]$, making an a-priori prediction even on their sign is a non-trivial matter. The linear theory predicts that two acoustically driven bubbles oscillate in phase and attract each other when the driving frequency is greater or lower than both their resonance frequencies, while they oscillate in counter-phase and repel with a driving frequency in-between their resonance frequencies [15]. Thus, Bjerknes forces are expected to be attractive for bubbles of equal size $[10,11]$. As a first, qualitative statement, we can therefore expect the cluster-cluster interaction to be attractive (since the pits are identical, the bubbles of each cluster should have similar sizes). Hence, the fact that clusters merge only above a certain threshold suggests that the pit-cluster interaction is also attractive, and that the cluster-cluster interaction must overcome the pit-cluster one to achieve cluster merging. However, it has been proved by a number of authors that the sign of the Bjerknes forces can be reversed due to several mechanisms neglected by the classical linear theory, such as the effect of secondary harmonics $[9,12]$, the resonance-like behavior of small bubbles (below their resonance size) near the dynamic Blake threshold [16] and viscous effects during translational motion [17]. Several studies of two bubbles interacting in a strong acoustic field have also shown that bubbles oscillating nonlinearly can form a bound pair with a steady spacing rather than collide and coalesce, as linear Bjerknes theory would predict [18-21]. Therefore caution is required when one wishes to understand the behavior of interacting clusters of bubbles.

In the present work we will investigate the Bjerknes forces acting upon the clus- 


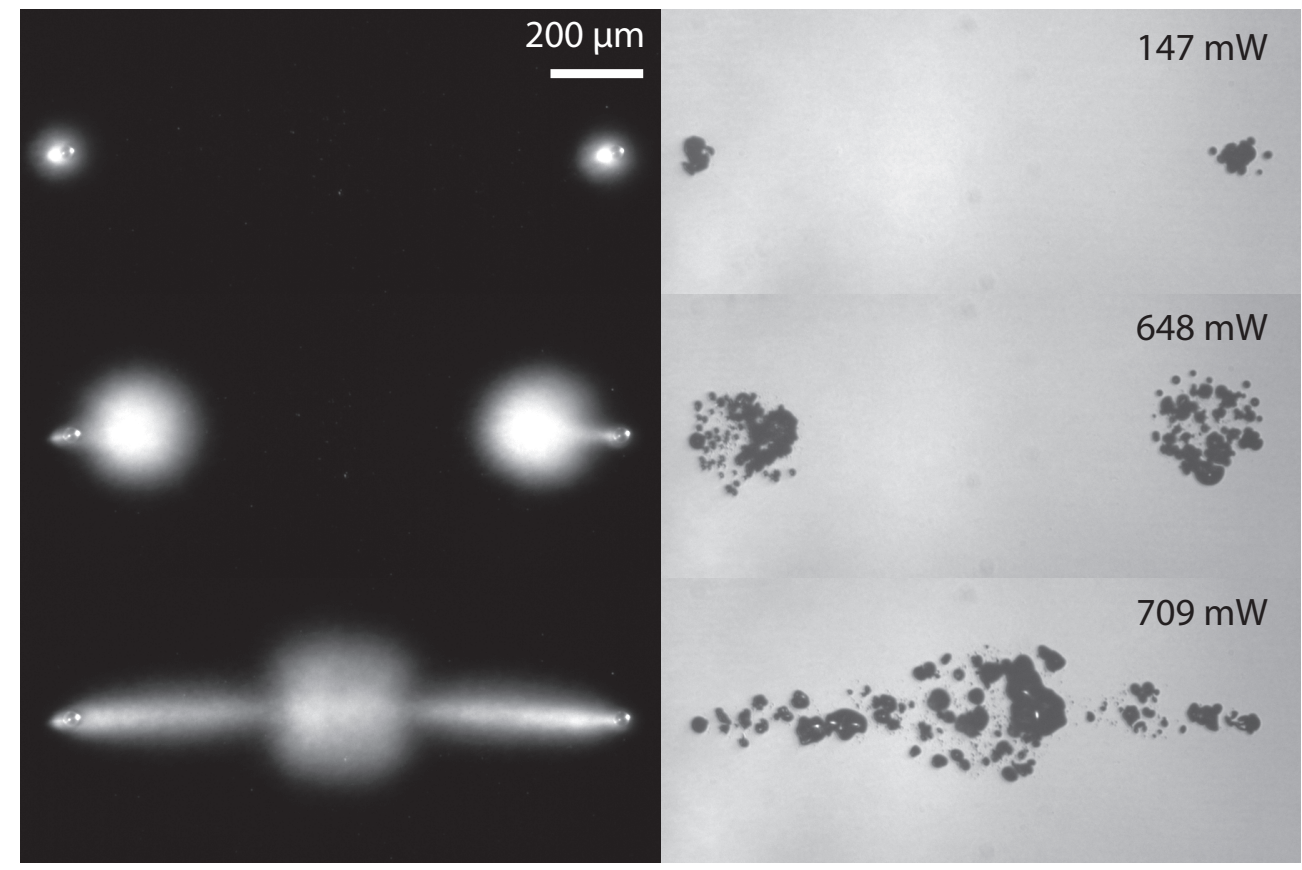

Figure 8.1: Example of the three different behaviors of the clusters observed in experiments, at increasing applied power: clusters close to the pits from which their bubbles originated (behavior 1, top), clusters pointing toward the center (behavior 2, middle), and clusters migrating toward the center (behavior 3, bottom). The left column was recorded at normal speed and represents therefore a time average, while the right column shows single snapshots taken with 7 ns exposure time. The experimental conditions are the same described in Ref. [2]. 


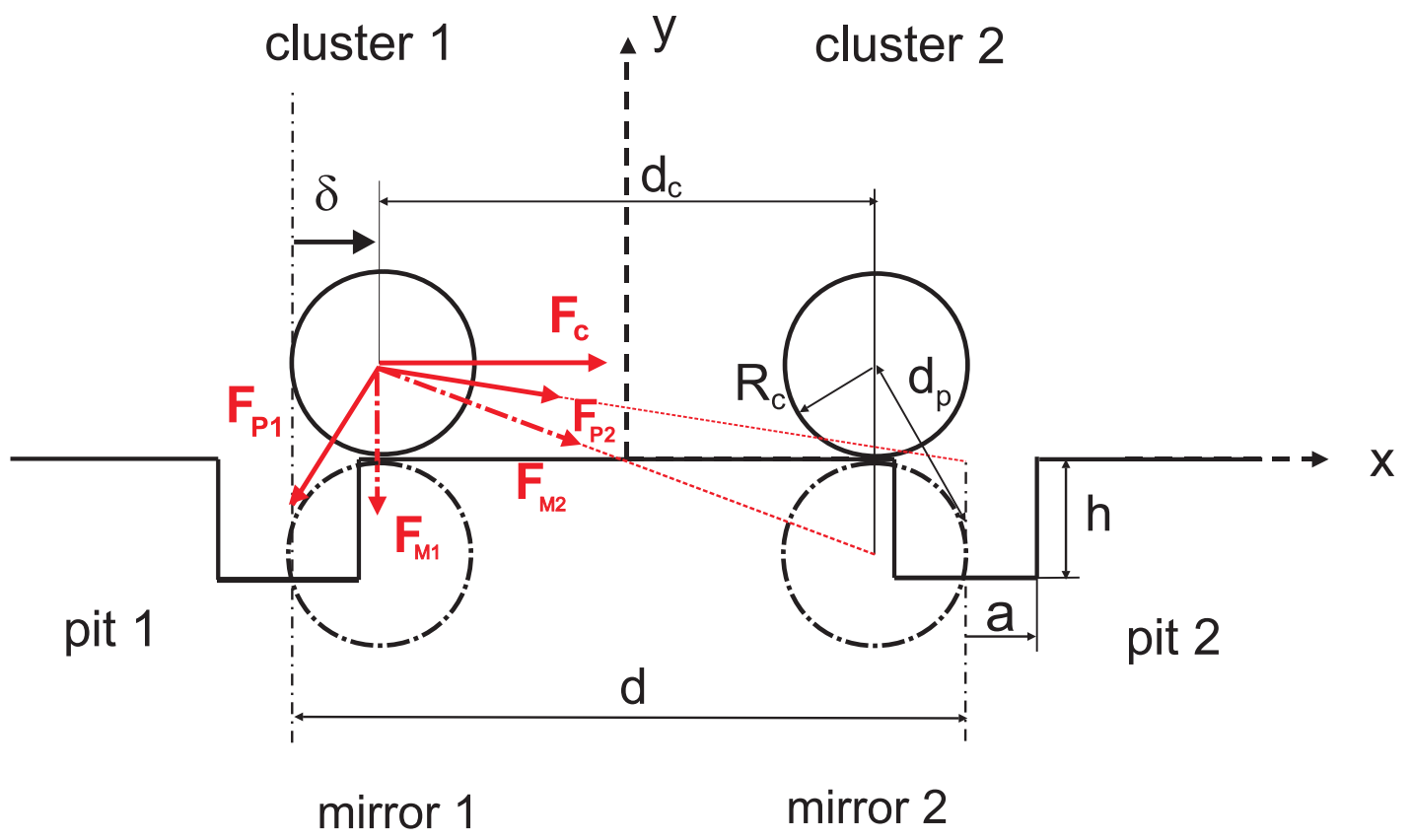

Figure 8.2: Sketch of the secondary Bjerknes forces acting upon the bubble and introduction of our employed notation. $F_{c}$ is the force from cluster $2, F_{p 1,2}$ are forces from the pits and $F_{m 1,2}$ are the forces from the mirror clusters.

ters and their dependence on different parameters, such as the size and the number of the bubbles, the size of the clusters, the distance between the pits and the driving pressure. We will show the influence of these forces upon the bubble dynamics and the radical production and will address the conditions required for the different transitions, thus providing practical indications for efficient sonochemical sonoreactors design, where one wishes to have the highest possible number of non-interacting micropits.

\subsection{Model}

In the experiment of Ref. [2] (Chap. 3), the bubble population is quite polydisperse. In top view, clusters appear as diffuse circles of radius $R_{c}$ (Fig. 8.1); a side view reveals that they are in contact with the substrate and have roughly an elliptic shape (Fig. 7 of Ref. [2]) with a long axis parallel to the substrate, and not much larger than 
the short axis. To simplify the problem, we will assume that each cluster is a sphere of radius $R_{c}$ in tangential contact with the substrate and we will neglect polydispersity. We will consider $R_{c}$ as time-invariant for simplicity reasons, although in reality this holds only on average, for a give applied power, but not in a rigorous sense. We will also assume that all the bubbles have the same radius $R(t)$ and that the two cluster have the same number of bubbles $N$, constant in time.

Each individual bubble in a cluster experiences acoustic interactions from the pits, from the neighboring bubbles of the same cluster, from the bubbles of the other cluster, and from all image bubbles, given the presence of the hard silicon substrate (see Fig. 8.2). We describe the behavior of a single bubble belonging to one of the clusters by extending the model previously developed in Refs. [22-24] and validated in [25], to incorporate the effect of the secondary Bjerknes forces upon the pulsation of the bubble. This model is then coupled with a static force balance in order to study the switch between the different conditions observed experimentally (see Fig. 8.1).

The model that we adopt for the single bubble is an ODE model based on the assumptions that the gas inside the bubble is a perfect gas and that the bubble has a uniform temperature and pressure. The temperature evolution is derived from the energy equation. Heat and mass transfer are treated with a boundary layer approximation [22-24]. Evaporation/condensation phenomena are kept into account, as well as the variation of the transport parameters due to compositional changes of the mixture. A list of 45 chemical reactions is included, with their temperature dependent chemical kinetics, governed by Arrhenius law. We refer the reader to [24] for a detailed description of these parts of the model, and we concentrate in the following upon the treatment of the Bjerknes forces.

The radial dynamics of a bubble belonging to cluster 1 is described by means of a modified Rayleigh-Plesset equation, keeping into account the effect of the secondary Bjerknes forces on the radial pulsation $[14,26]$ :

$$
\begin{aligned}
\left(1-\frac{\dot{R}}{c}\right) R \ddot{R}+\frac{3}{2}\left(1-\frac{\dot{R}}{3 c}\right) \dot{R}^{2} \\
\quad=\frac{1}{\rho}\left(1+\frac{\dot{R}}{c}\right)\left(p-p_{\infty}-P(t)\right)+\frac{R \dot{p}}{\rho c}-\frac{4 v \dot{R}}{R}-\frac{2 \sigma}{\rho R}-T_{B j} .
\end{aligned}
$$

Here the dots are used for time derivatives, $R$ is the radius of the bubble, $c$ is the speed of sound, $\rho$ is the density of the liquid, $v$ its kinematic viscosity, $\sigma$ is the surface tension, $p_{\infty}$ the static pressure and $P(t)=P_{a} \cos \omega t$ is the acoustic driving pressure, with $P_{a}$ the driving amplitude, $f=\omega / 2 \pi$ the frequency and $\tau=1 / f$ the period of the driving. $T_{B j}$ is a coupling term expressing the effect of the interaction 
with the other bubbles, both real and imaginary, and the pits

$$
T_{B j}=T_{c 1}+T_{c 2}+T_{m 1}+T_{m 2}+T_{p 1}+T_{p 2} .
$$

$T_{c 1}$ and $T_{c 2}$ are the coupling terms with the bubbles of the same cluster and the other cluster respectively, $T_{m 1,2}$ are the coupling terms with the two mirror clusters, $T_{p 1,2}$ are the coupling terms with the two pits.

The coupling term $T_{2 \rightarrow 1}$ between two isolated bubbles, describing the influence of bubble 2 on the radial oscillations of bubble 1, can be written as [16]

$$
T_{2 \rightarrow 1}=\frac{1}{d}\left(R_{2}^{2} \ddot{R}_{2}+2 R_{2} \dot{R}_{2}^{2}\right) .
$$

Therefore, the coupling term between one bubble $i$ and the other bubbles of the cloud to which it belongs becomes

$$
T_{c 1}=\sum_{j \neq i} \frac{R_{j}^{2} \ddot{R}_{j}+2 R_{j} \dot{R}_{j}^{2}}{r_{i j}},
$$

in which $r_{i j}$ is the distance between bubbles $j$ and $i$. Following the approximation of Yasui et al. [14], i.e. neglecting polydispersity and assuming that the cluster has constant density, we get:

$$
T_{c 1} \simeq 2 \pi n R_{c}^{2}\left(R^{2} \ddot{R}+2 R \dot{R}^{2}\right),
$$

where $R_{c}$ is the cluster radius and $n$ is the number density of bubbles, $n \simeq 3 N / 4 \pi R_{c}^{3}$.

The coupling term between the considered bubble of cluster 1 and all the bubbles of cluster 2 is expressed as in Ref. [14]

$$
T_{c 2} \simeq \frac{N}{d_{c}}\left(R^{2} \ddot{R}+2 R \dot{R}^{2}\right),
$$

where $d_{c}$ is the distance between the two clouds. This is valid as long as $d_{c} \gg R_{c}$. The coupling terms $T_{m 1,2}$ with the two mirror clusters are expressed in a similar way.

In order to model the interaction of the bubble with the pit, we consider the pit as an effective bubble with the same resonant frequency $\omega_{p}$ and damping coefficient $\beta$ as the pit. Although the pit is not a spherical bubble, and may oscillate in a nonlinear fashion, we will treat it as an harmonic oscillator. This assumption is acceptable, as long as we showed in Chap. 7 that, in the considered parametric range, large amplitude oscillations present an overall behavior similar to small amplitude oscillations, 
with a slightly lower damping but the same resonance frequency. Hence, we consider the pit like a spherical bubble of radius at rest $R_{p}^{0}$, such that $\omega_{p}^{2}=3 p_{\infty} / \rho\left(R_{p}^{0}\right)^{2}$, experiencing linear oscillations [27]: $R_{p}=R_{p}^{0}\left(1+x_{p}\right)$ with:

$$
\ddot{x}_{p}+2 \beta \dot{x}_{p}+\omega_{p}^{2} x_{p}=-\frac{p_{a}(t)}{\rho\left(R_{p}^{0}\right)^{2}} .
$$

The values of $\omega_{p}$ and $\beta$ are taken from the recent results of Gelderblom et al. [28]. These authors have computed the acoustic response of a gas pocket entrapped in a pit, providing its eigenfrequency and damping coefficient, in two limits: potential flow, and unsteady Stokes equation. For a cylindrical pit of radius $a$ and height $h$, the results mainly depend on the parameter $P=\kappa a^{2} p_{0}^{\infty} / h \sigma$. In the experiments of $[1,2], a=15 \mu \mathrm{m}, h=10 \mu \mathrm{m}, p_{0}^{\infty}=10^{5} \mathrm{~Pa}$ and $\sigma=0.07 \mathrm{~N} / \mathrm{m}$. The temperature was controlled providing an isothermal behavior within a precision of $1 \mathrm{~K}$ [2] and therefore $\kappa=1$. Hence we compute $P=32$, from which $\hat{\omega}_{p}=5.82$ and $\hat{\beta}=0.26$ in the Stokes regime, and $\hat{\omega}_{p}=6.04$ and $\hat{\beta}=0.20$ in the potential regime. The dimensionless frequency is defined as $\hat{\omega}_{p}=\omega_{p} \sqrt{\rho a^{3} / \sigma}$, with a rescaling angular frequency $\sqrt{\sigma / \rho a^{3}}=1.46 \times 10^{5} \mathrm{rad} / \mathrm{s}$. Hence, taking $\hat{\omega}_{p}=6$, the resonance frequency of a pit equals $f_{p}=143 \mathrm{kHz}$, with $f_{p}=\omega_{p} / 2 \pi$. In the experiments of $[1,2]$, it is not clear which of the two regimes, potential or Stokes, applies best, but it is seen that the numerical values of eigenfrequency and damping differ by only $10 \%$. Moreover, in the numerical work of Chap. 8 it was shown that in the intermediate regime where both inertia and viscosity are present, the overall behavior of the pit is generally closer to the Stokes regime. So, we use hereby the results related to the Stokes regime for the damping and we take $\beta=3.8 \times 10^{5} \mathrm{~s}^{-1}$.

By substituting $R_{p}(t)$ inside (8.3), the coupling term between the considered bubble of cluster 1 and pit 1 can be expressed as

$$
T_{p 1}=\frac{1}{d_{p 1}}\left(R_{p}^{0}\right)^{3}\left(1+x_{p}\right)\left(2 \dot{x}_{p}^{2}+x_{p} \ddot{x}_{p}+\ddot{x}_{p}\right),
$$

where $d_{p 1}$ is the distance between the bubble and the pit. The coupling term $T_{p 2}$ between the bubble of cluster 1 and pit 2 is expressed correspondingly.

We now turn to the forces experienced by the clusters (see Fig. 8.2). As we want to study the transition between behavior 1 and behavior 2, we will focus on the forces acting on the horizontal plane. Hence, we will neglect both the buoyancy and the primary Bjerknes force, which is directed in the vertical direction, because the driving pressure is a standing wave with a anti-node on the substrate and a node at the free water-air surface [29]. 
In a static equilibrium condition, all the forces acting upon a bubble of cluster 1 with an horizontal component are secondary Bjerknes forces. Such forces are exerted by the other cluster $\vec{F}_{c}$, by the mirror clusters $\vec{F}_{m 1,2}$ and by the pits $\vec{F}_{p 1,2}$. In order to derive them, we first consider two oscillating bubbles, of volume $\mathscr{V}_{1}$ and $\mathscr{V}_{2}$, separated by a distance $d$ much greater than their radii; then bubble 1 experiences a force equal to [16]:

$$
\vec{F}_{2 \rightarrow 1}=\frac{\rho \ddot{\mathscr{V}}_{2} \mathscr{V}_{1}}{4 \pi d^{2}} \hat{e}_{2 \rightarrow 1},
$$

with $\hat{e}_{2 \rightarrow 1}$ the unit vector pointing from bubble 2 to bubble 1 . For bubbles of the same radius $R$, this reduces to $\vec{F}_{2 \rightarrow 1}=4 \pi \rho R^{3}\left(R^{2} \ddot{R}+2 R \dot{R}^{2}\right) \hat{e}_{2 \rightarrow 1} / 3 d^{2}$. Let us first notice that the forces between bubbles pertaining to the same cluster are responsible of the cluster cohesion, but are irrelevant to the interaction between different clusters; hence, we neglect them to assess the stability of behavior 1 . Assuming that $R_{c} \ll d_{c}$, each bubble of cluster 1 experiences from cluster 2 a force equal to [14, 26]:

$$
\vec{F}_{c}=\frac{4 \pi \rho N R^{3}\left(R^{2} \ddot{R}+2 R \dot{R}^{2}\right)}{3 d_{c}^{2}} \hat{e}_{2 \rightarrow 1} .
$$

As $d_{c}=d-2 \delta$ (Fig. 8.2), the horizontal component of $\vec{F}_{c}$ is given by

$$
F_{c, x}=\frac{A_{c}}{(d-2 \delta)^{2}}
$$

with $A_{c}=4 \pi \rho N R^{3}\left(R^{2} \ddot{R}+2 R \dot{R}^{2}\right) / 3$. The forces acting on each bubble of cluster 1 from the mirror clusters, namely $\vec{F}_{m 1}$ and $\vec{F}_{m 2}$, and their horizontal components can be expressed in a similar way.

The secondary Bjerknes force acting over each bubble of cluster 1 from pit 1 is found by substituting the volume of the equivalent bubble corresponding to the pit $\mathscr{V}_{p}=4 \pi R_{p}^{3} / 3$ into Eq. (8.9),

$$
\vec{F}_{p 1}=\frac{4}{3} \frac{\pi \rho\left(R_{p}^{0}\right)^{3}}{d_{p}^{2}} R^{3}\left(1+x_{p}\right)\left[2 \dot{x}_{p}^{2}+\ddot{x}_{p}\left(1+x_{p}\right)\right] \hat{e}_{p}
$$

Here $\hat{e}_{p}$ is the unit vector pointing from the pit to the bubble and $d_{p}^{2}=h_{p}^{2}+\delta^{2}$, with $h_{p}=R_{c}+h / 2$. Even if $d_{p}$ depends on the location of the bubble within its cluster, we will take $d_{p}$ as the distance between the pit and the center of the cluster; in practice, off-centered bubbles within the cluster will experience pit interaction of a different magnitude, but this will be compensated by the interaction with the other bubbles forming the cluster. Given $\hat{e}_{p} \cdot \hat{e}_{x}=\delta / d_{p 1}$, the horizontal component of $F_{p 1}$ can be 
calculated from (8.12) as

$$
F_{p 1, x}=A_{p 1} \frac{\delta}{\left[h_{p}^{2}+\delta^{2}\right]^{3 / 2}},
$$

with $A_{p 1}=4 \pi \rho\left(R_{p}^{0}\right)^{3} R^{3}\left(1+x_{p}\right)\left[2 \dot{x}_{p}^{2}+\ddot{x}_{p}\left(1+x_{p}\right)\right] / 3$. The force $F_{p 2}$ acting on each bubble of cluster 1 from pit 2 and its horizontal component are calculated similarly.

\subsection{Results}

\subsubsection{Transition from individual clusters to merging clusters}

Fernández-Rivas et al. [2] provide an experimental characterization of the number and size of the bubbles present in the cluster for one, two and three pits, and for three different powers. They show that the number of bubbles $N$ increases at increasing power, and that both the average bubble radius $(R \simeq 10 \mu \mathrm{m})$ and the most probable radius $(R \simeq 3 \mu \mathrm{m}$ ) have no significant dependence on the power and on the number of pits. According to (8.10) and (8.12), in first order, i.e. neglecting the effect of the other bubbles on $\mathrm{R}(\mathrm{t})$, the cluster-cluster force is proportional to $N$, whereas the pit-cluster force does not depend on $N$. The threshold pressure for merging may thus originate either from the fact that $N$ increases at increasing power or from higher order effects of bubble oscillations on the secondary Bjerknes forces, contained inside the terms $A_{p}$ and $A_{c}$.

In order to investigate the transitions between the three different behaviors found in experiments (see Fig. 8.1), we adopt a quasi-static "adiabatic" approach: we take $\delta$ as a constant over time, representing the displacement of the bubble and therefore of the cloud from the initial equilibrium position. At each instant, we calculate the horizontal components of $\vec{F}_{c}, \vec{F}_{p 1,2}$ and $\vec{F}_{m 1,2}$. We stop the calculation after one cycle, to match the experimental conditions of Ref. [2], where the bubbles did not survive after the first collapse. We perform the time averages of the forces over the whole cycle and we verify whether the following holds

$$
\left\langle F_{x}^{*}\right\rangle>\left\langle F_{p 1, x}\right\rangle
$$

where $\left\langle F_{x}^{*}\right\rangle=\left\langle F_{c}\right\rangle+\left\langle F_{p 2, x}\right\rangle+\left\langle F_{m 2, x}\right\rangle$ is the sum of the forces attracting the clusters towards each other and $\langle\cdot\rangle$ denotes the time average over the first acoustic cycle.

In order to study the transition between behavior 1 and behavior 2, i.e. the inception of the motion, we consider a small initial horizontal displacement $\delta=R_{0}$ of the bubble from the pit axes and therefore from its rest conditions. The motion 


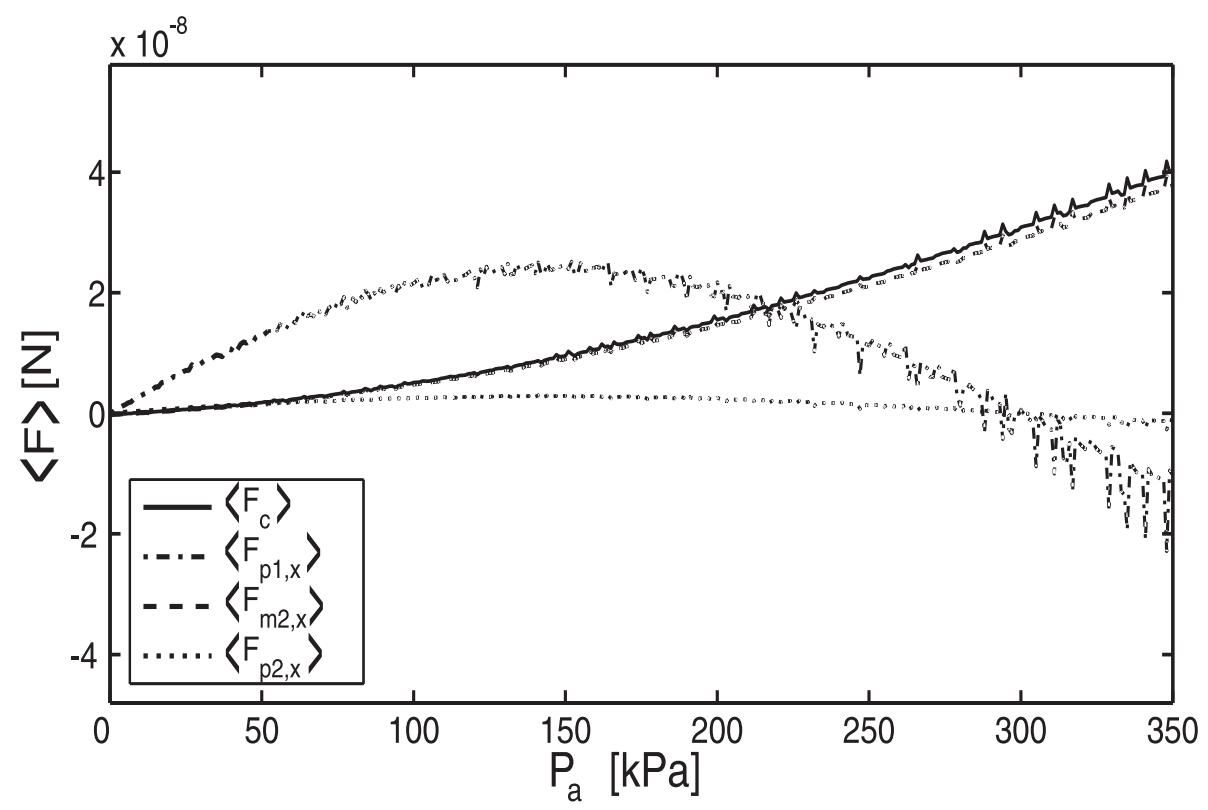

Figure 8.3: Forces acting upon a bubble of cluster 1 with an initial displacement $\delta=R_{0}$ respect to its unperturbed condition. Parameters: $R_{c}=100 \mu \mathrm{m}, N=100$, $d=1000 \mu \mathrm{m}, R_{0}=10 \mu \mathrm{m}, f=200 \mathrm{kHz}$. The cluster-cluster forces are always attractive, while the pit-cluster forces become repulsive at high driving, thus favoring the inception of the motion.

starts once Eq. (8.14) holds. However, this does not imply that the bubble will eventually reach the center of the pit array, as we will show below. An example of forces acting upon a bubble with an initial displacement of $\delta=R_{0}$ is given on Fig. 8.3. Sign inspection shows that the cluster-cluster forces both with the real and the mirror cluster 2 are always attractive, while the pit-clusters forces become repulsive at high driving pressure. Above pressures of $150 \mathrm{kPa}$ the data become noisy, probably due to the nonlinearity of the problem.

In order to study the transition between behavior 2 and behavior 3, i.e. the merging of the clusters, we consider both $F_{x}^{*}$ and $F_{p 1, x}$ as a function of $\delta$. The transition occurs once Eq. (8.14) holds at all $\delta$. As a function of $\delta,\left\langle F_{p 1, x}\right\rangle$ has a maximum, generally (but not always) corresponding to the point $\bar{\delta}$ where the pit traps the cluster. The transition is graphically sketched on Fig. 8.4. The blue line corresponds to merging clusters (behavior 3 ); the green line corresponds to the situation where $\left\langle F_{x}^{*}\right\rangle$ 


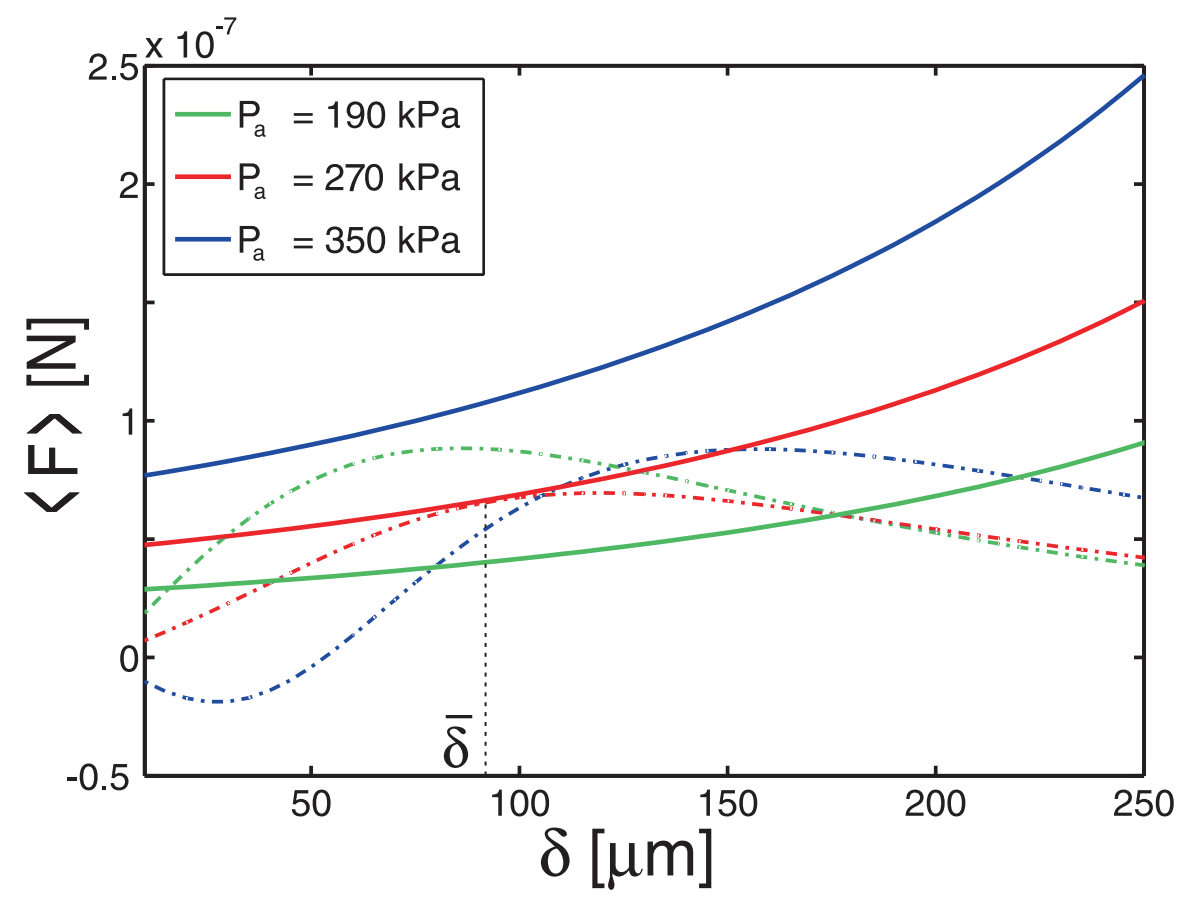

Figure 8.4: Average forces $\left\langle F_{x}^{*}\right\rangle$ (solid) and $\left\langle F_{p 1, x}\right\rangle$ (dash-dot) acting upon a bubble of cluster 1, over one acoustic cycle, as function of $\delta$ for $R_{0}=10 \mu \mathrm{m}, N=100, R_{C}$ $=100 \mu \mathrm{m}, d=1000 \mu \mathrm{m}, f=200 \mathrm{kHz}$. In green, low pressure case: $\left\langle F_{x}^{*}\right\rangle$ is too weak to pass the barrier constituted by the restoring force $\left\langle F_{p 1, x}\right\rangle$; the cluster remains attached to its pit, with a small displacement given by the first intersection of the two curves. In blue, high pressure case: $\left.\left\langle F_{x}^{*}\right\rangle\right\rangle\left\langle F_{p 1, x}\right\rangle$, and the two clusters merge. In red, critical case, defining the transition between both behaviors. $\bar{\delta}$ represents the maximum displacement of the cluster before transition.

is strong enough to induce the inception of the motion but too weak to overcome the barrier constituted by the restoring force of the pit. Therefore the cluster remains attached to its pit, with a small displacement given by the intersection of the two curves (behavior 2). The red line represents the transition between behavior 2 and behavior 3, and the corresponding pressure amplitude will be denoted from now on as $\bar{P}_{a}$.

In Fig. 8.5 we plot the driving pressure required for the two transitions, namely the one for cluster 1 to start moving (dash-dotted line) and the one to overcome the trapping force of the pit (solid line), as a function of the distance between the pits $d$. 


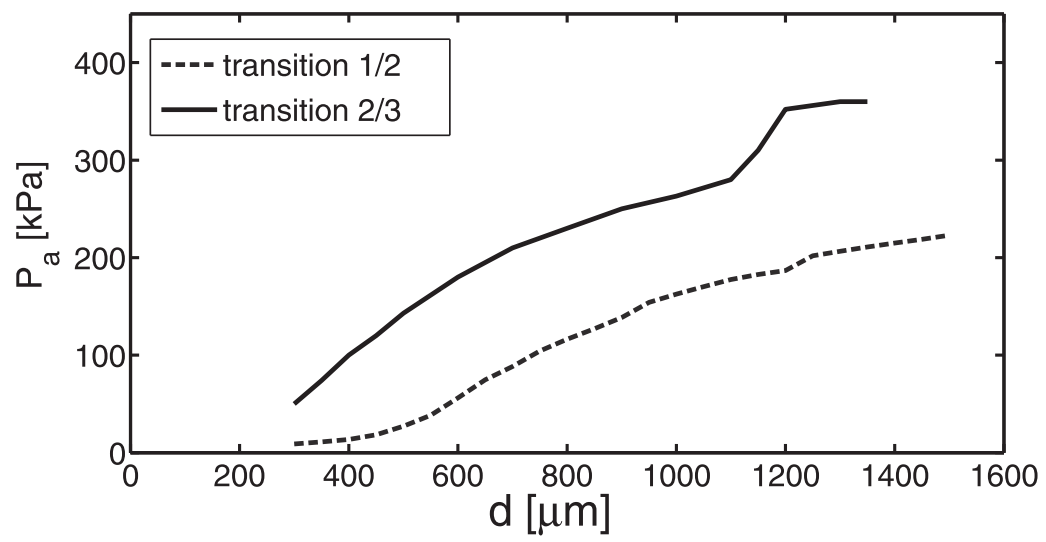

Figure 8.5: Calculated driving pressure for transition from behavior 1 to 2, i.e. the inception of the motion (dash) and from behavior 2 to 3, i.e. the merging of the clusters (solid), as function of the distance between the pits for a cluster with $R_{0}=$ $10 \mu \mathrm{m}, N=100, R_{c}=100 \mu \mathrm{m}$, driven at $f=200 \mathrm{kHz}$. When the pits are too far apart $(d \gtrsim 1350 \mu \mathrm{m})$, no merging is possible.

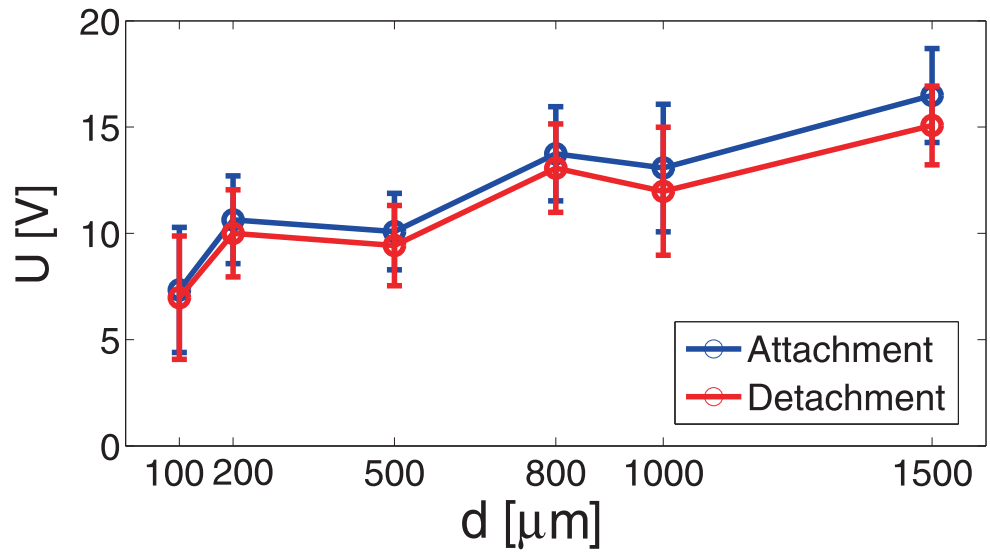

Figure 8.6: Electric potential applied to the piezo when the clusters start to merge, at increasing power (blue), and when they detach going back to their own pits, at decreasing power (red), as function of the distance between the pits, in the experiment described in Ref.[2]. Increasing voltage corresponds to increasing $P_{a}$. For $d>1500 \mu \mathrm{m}$ no merging was observed, no matter $P_{a}$. 


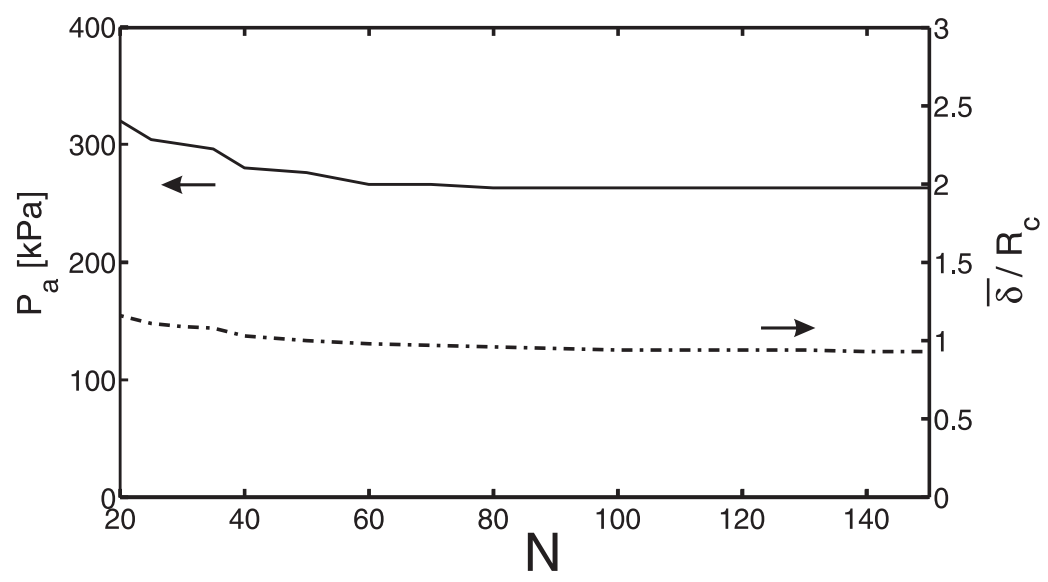

Figure 8.7: Driving pressure (solid) and maximum displacement of the cluster (dashdot) at transition from behavior 2 to behavior 3, as function of the number of bubbles. Parameters: $R_{0}=10 \mu \mathrm{m}, R_{c}=100 \mu \mathrm{m}, d=1000 \mu \mathrm{m}, f=200 \mathrm{kHz}$. For $N>50$ they become both almost invariant to a further increase of $N$.

According to the experimental conditions of Ref. [2], we consider two clusters with $R_{c}=100 \mu \mathrm{m}, N=100, R_{0}=10 \mu \mathrm{m}$. As the distance between the pits increases, a higher $P_{a}$ is required for merging. For $d=1000 \mu \mathrm{m}$, we calculated that the transition occurs at $\bar{\delta}=94 \mu \mathrm{m}$, with $\bar{P}_{a}=270 \mathrm{kPa}$. In the experiments, the maximum displacement of the clusters before they detach from the pit and started coalescing was $\bar{\delta} \sim R_{c}$ (see Fig. 8.1). Moreover, three pressure values have been measured, corresponding to the three different levels of of the applied power (low, medium and high), $165 \mathrm{kPa}, 225 \mathrm{kPa}$ and $350 \mathrm{kPa}$ respectively. The transition occurred between $225 \mathrm{kPa}$ and $350 \mathrm{kPa}$ [2]. Despite the approximations of the model, such as the equal size and monodispersity of the bubbles inside the cluster and the time-invariance of both the number of the bubbles and the size of the clusters, we remark that these values are extremely close to the experimental ones.

Also as a function of the distance between the pits, the numerical results reproduce the same trend found in experiments: when $d$ increases, so does $\bar{P}_{a}$, until a limiting value of $d$, where no merging is possible anymore. In the experiments, this limit was found at $d=1500 \mu \mathrm{m}$, in the simulation at $d=1350 \mu \mathrm{m}$. Once again, the agreement with the model is remarkably good. The trapping distance $\bar{\delta}$ increases with $d$.

In Fig. 8.6, we show the voltage applied to the piezo when the clusters merge, at increasing applied power (blue) and when the clusters detach, coming back to their 


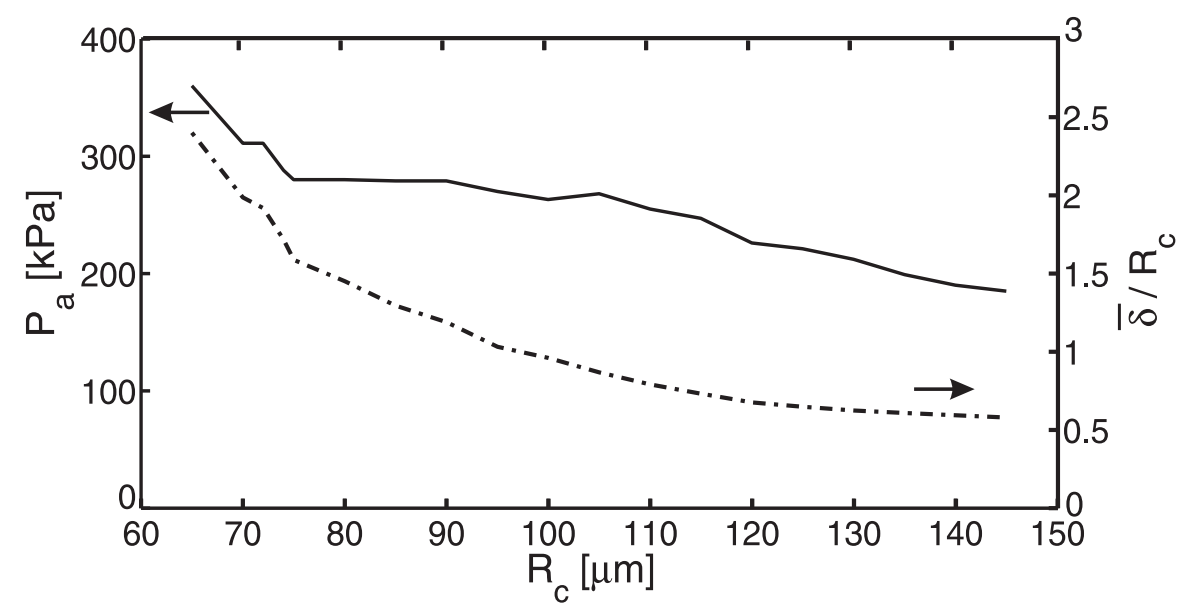

Figure 8.8: Driving pressure (solid) and maximum displacement of the cluster (dashdot) at transition from behavior 2 to behavior 3 , as function of the radius of the cluster. Parameters: $R_{0}=10 \mu \mathrm{m}, N=100, d=1000 \mu \mathrm{m}, f=200 \mathrm{kHz}$. For bigger clusters the $P_{a}$ required for merging is lower, as well as the maximum displacement of the cluster.

own pits, at decreasing applied power (red), for the same setup and experimental conditions of Ref. [2]. As the applied voltage is directly connected to the driving pressure $P_{a}$, we can conclude that the driving pressure required for merging is higher than the one required to detach the clusters. The theoretical investigation of this hysteretic behavior is beyond the scope of the present paper and should be addressed in future works.

For a given $R_{0}$ and $R_{c}, \bar{P}_{a}$ has a very slight dependence on the number of bubbles and increasing $N$ reduces $\bar{P}_{a}$ only until a certain value of $N$. With $R_{0}=10 \mu \mathrm{m}, R_{c}$ $=100$ and $d=1000 \mu \mathrm{m}$ this happens for $N<50$ (see Fig. 8.7). For clusters with a higher number of bubbles, a further increase of $N$ does not imply a further decrease of $\bar{P}_{a}$. This means that the number of bubbles itself is not what determines the transition between separated clusters and merging clusters. Thus, we can conclude that, at medium and high power, the phenomenon is governed by nonlinear oscillations effects contained inside $A_{p}$ and $A_{c}$ in Eqs. (8.11) and (8.13).

However, the number of bubbles can still have an indirect influence on the transition: given a certain $R_{0}$ and $N$, both $\bar{P}_{a}$ and $\bar{\delta}$ are higher when the cloud radius $R_{c}$ is smaller (see Fig. 8.8). As nonlinear oscillating bubbles tend to form stable pairs without coalescing, i.e. the bubbles remain at a certain equilibrium distance from 


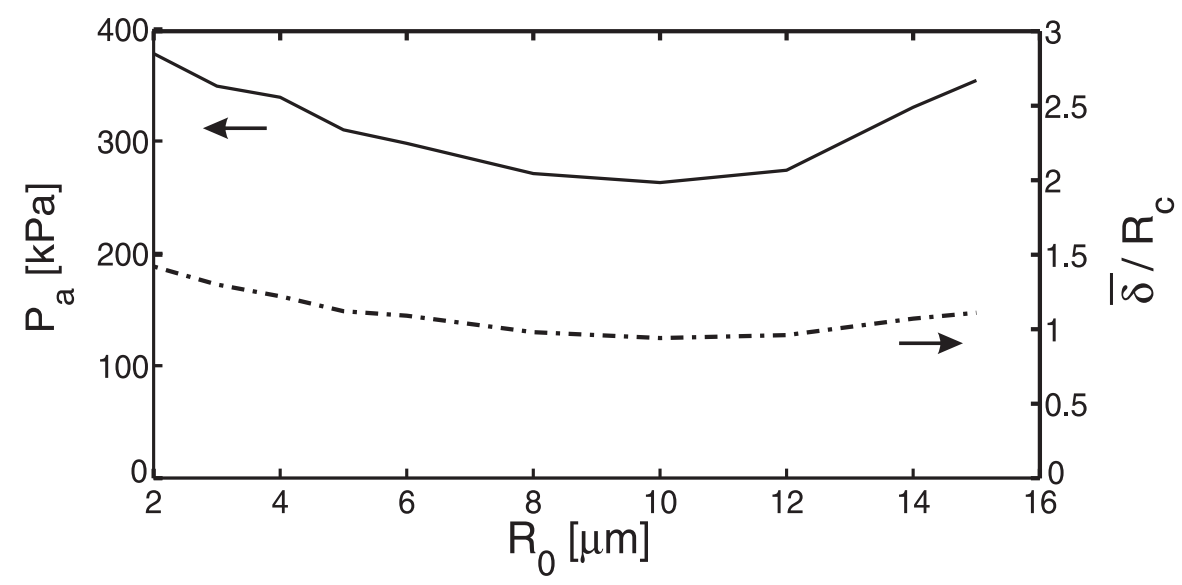

Figure 8.9: Driving pressure (solid) and maximum displacement of the cluster (dashdot) at transition from behavior 2 to behavior 3 , as function of the radius of the bubbles. Parameters: $R_{c}=100 \mu \mathrm{m}, N=100, d=1000 \mu \mathrm{m}, f=200 \mathrm{kHz}$. The $P_{a}$ required for merging presents a minimum with bubbles of $10 \mu \mathrm{m}$, as well as the maximum displacement, which is however almost invariant with $R_{0}$.

each other [18-21], we can expect that an increase in the number of bubbles will also lead to an increase in the cluster size and therefore to a lower $P_{a}$ at transition.

The dependence of the pressure amplitude for clusters merging on the bubble size is non-monotonic. In a range from $R_{0}=3$ to $15 \mu \mathrm{m}$, the clusters requiring a lower $\bar{P}_{a}$ to escape from the pit region are those with $R_{0}=10 \mu \mathrm{m}$ (see Fig.8.9). Therefore we can assume that these bubbles will also be the ones to initiate the merging. Nevertheless, the inclusion of inertia and drag, excluded from the present approach, could shift this minimum towards lower values of $R_{0}$ and it should be addressed in a future paper.

\subsubsection{Radical production}

In order to investigate the effects of the Bjerknes forces on the radical production, we consider a cluster with $R_{c}=100 \mu \mathrm{m}, N=100, d=1000 \mu \mathrm{m}, R_{0}=3 \mu \mathrm{m}$, driven at $f$ $=200 \mathrm{kHz}$ and $P_{a}=270 \mathrm{kHz}$. In Fig. 8.10 we show the radial and thermal evolution of a bubble of such a cluster. Including the Bjerknes forces (solid line) has the same effect of adding some damping to the system, as it leads to a lower expansion of the bubble [14] and therefore to a lower temperature at collapse respect to the case where Bjerknes forces are not included (solid-dashed line). As the radical production 

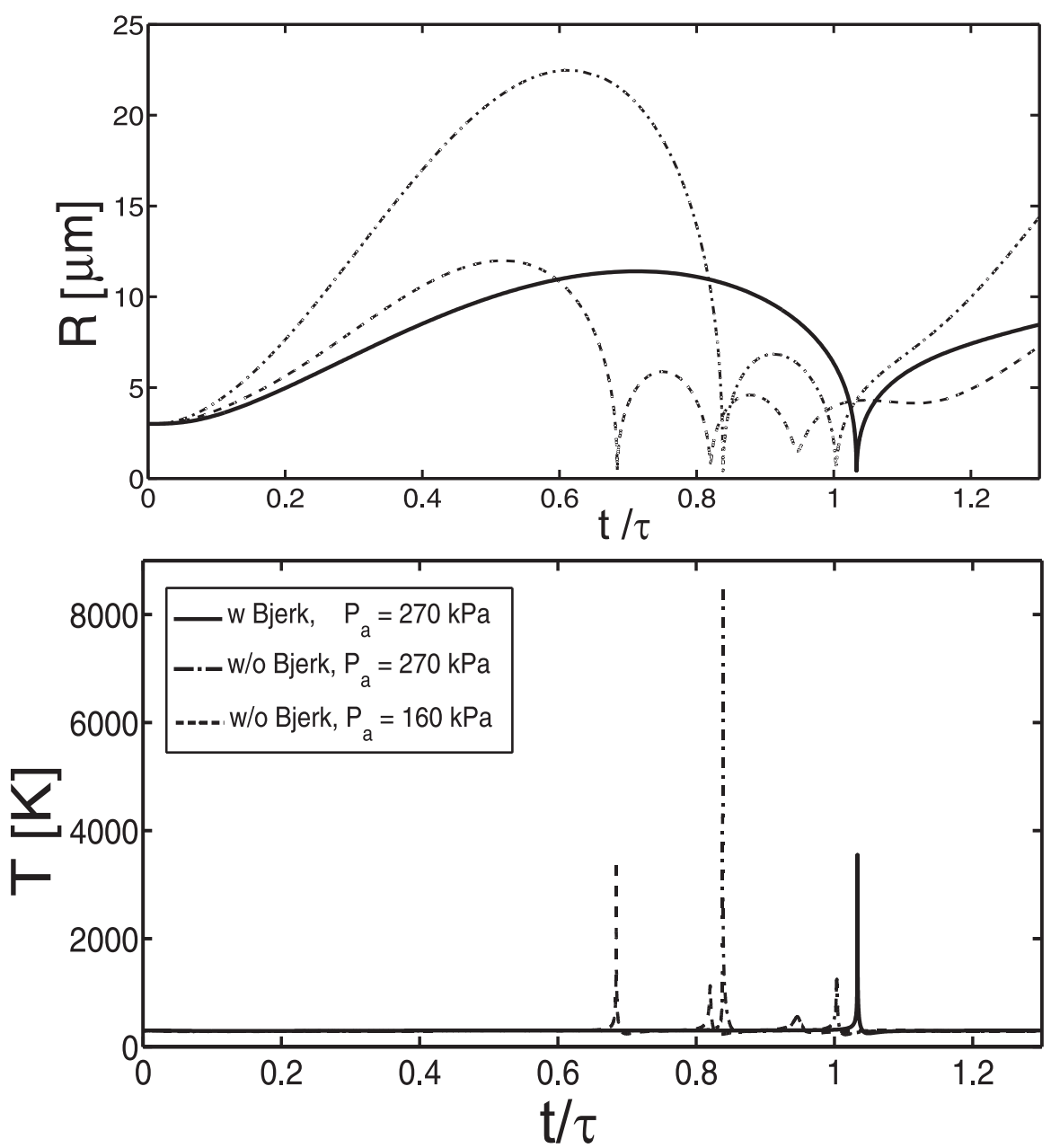

Figure 8.10: Radius vs time (top) and temperature vs time (bottom) curves, for a bubble belonging to cluster 1 . The driving amplitude corresponds to the calculated one for the transition between behavior 2 and behavior 3, both including (solid) and disregarding (dash-dot) the Bjerknes forces for $P_{a}=270 \mathrm{kPa}$. The dashed lines correspond to an isolated bubble, driven at $P_{a}=160 \mathrm{kPa}$, that equals the "effective pressure" deduced from the bubble dynamics disregarding Bjerknes forces, just before transition in Ref. [2]. Parameters: $R_{0}=3 \mu \mathrm{m}, R_{c}=100 \mu \mathrm{m}, N=100, f=$ $200 \mathrm{kHz}, d=1000 \mu \mathrm{m}, a=15 \mu \mathrm{m}, h=10 \mu \mathrm{m}$. 


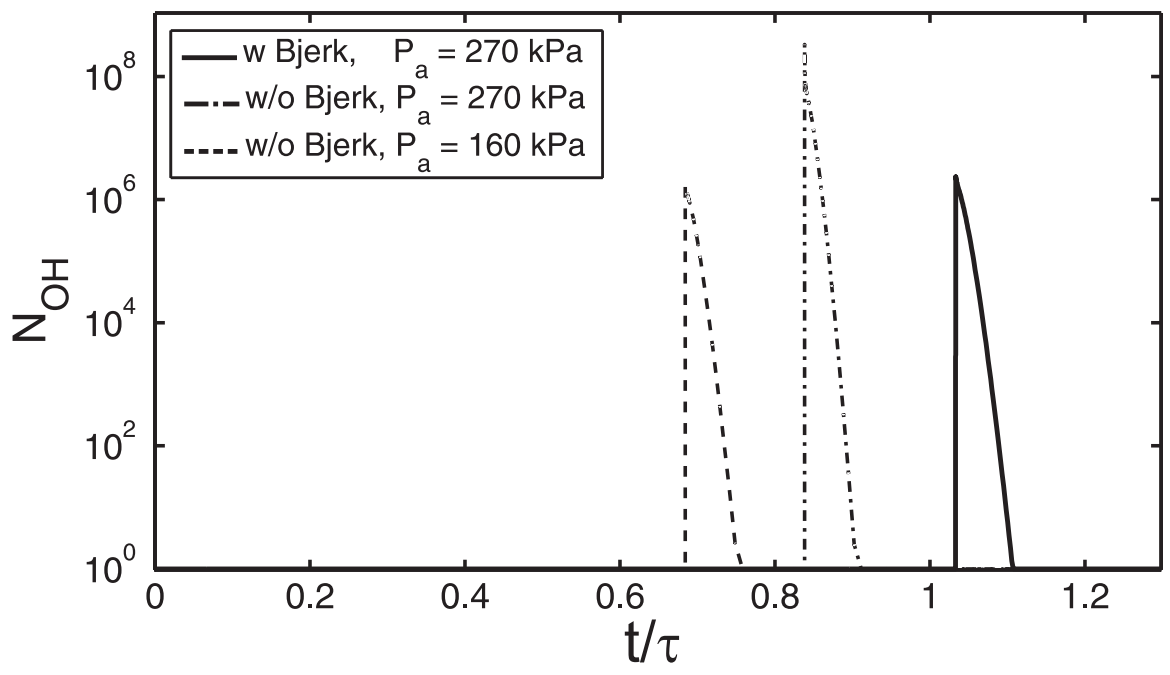

Figure 8.11: Number of $\mathrm{OH}$ radicals produced as function of time, for a bubble belonging to cluster 1 driven at $P_{a}=270 \mathrm{kPa}$, with (solid) and without (dash-dot) Bjerknes forces, and at $P_{a}=160 \mathrm{kPa}$ without Bjerknes forces (dash). Driving conditions and dimensions as in Fig. 8.10.

is related to the peak temperature through Arrhenius law, neglecting the Bjerknes forces induces an overestimate of the produced radicals (see Fig. 8.11). Moreover, the Bjerknes forces reduce the eigenfrequency of the bubble (see Fig. 8.10), and therefore induces a further reduction of the radical production, due to the lower number of collapses per unit time respect to the isolated bubble. From the theoretical point of view this reduction of the resonance frequency can be predicted using the standard approach for the calculation of the linear resonance frequency of a bubble [30, 31]. We rewrite the modified Rayleigh-Plesset equation (8.1) by neglecting the effects of liquid compressibility, surface tension and viscosity, under the hypothesis of linear oscillations. Considering just the effect of the other bubbles of the same cloud the new linear frequency will be such to satisfy $\omega_{0}^{2}=3 \kappa p_{\infty} /\left[\rho R_{0}^{2}\left(1+3 / 2 N R_{0} / R_{c}\right)\right]$. With $N$ varying between 10 and $100, N R_{0} / R_{c}$ varies between 1 and 10 . For $R_{0}=10 \mu \mathrm{m}$, we compute $f_{0}=326,304,206$, and $82 \mathrm{kHz}$, respectively for $N R / R_{c}=0$ (single bubble), 1,10 , and 100. Given the resonance frequency of the pit $f_{p}=143 \mathrm{kHz}$ and the driving frequency $f=200 \mathrm{kHz}$, in the linear regime, i.e. at low driving amplitude, an attractive pit-cluster force is expected, in agreement with what we found. However, due to nonlinearities, at high driving amplitude, the pit-cluster force can become repulsive [16, 21] (see Fig. 8.3). In Fig. 8.10 and in Fig. 8.11 we also show the 


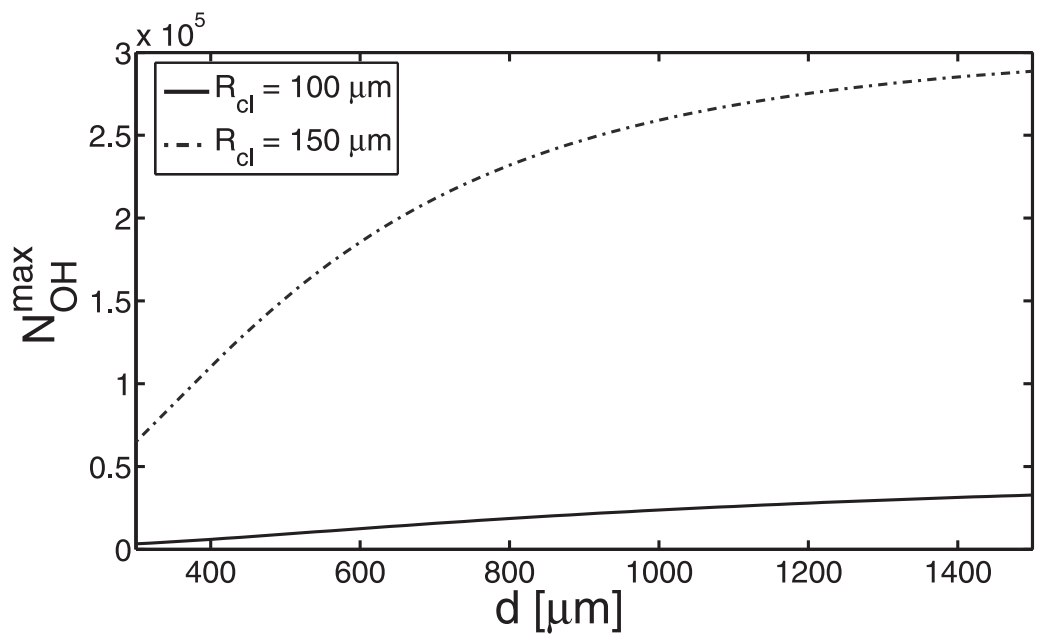

Figure 8.12: Maximum number of $\mathrm{OH}$ radicals in one acoustic cycle for a bubble of cluster 1 in the unperturbed position $(\delta=0 \mu \mathrm{m})$ as function of the distance between the pits, for different cluster sizes (as indicated in the legend). Parameters: $N=100$, $R_{0}=3 \mu \mathrm{m}, f=200 \mathrm{kHz}, P_{a}=170 \mathrm{kPa}$.

dynamic, thermal, and chemical evolution of the same bubble driven at $P_{a}=160 \mathrm{kPa}$, without the Bjerknes forces. This driving amplitude corresponds to the "effective pressure" that we calculated in our previous work for the two pits case, just before the merging occurred (see Fig. 21 in Ref.[2]). In that case the effective pressure was extracted from the experimental data by neglecting the Bjerknes forces and using the bubbles as a pressure sensor through their recorded dynamics. Although there are some differences between the radial evolution curve of a bubble driven at $P_{a}=270$ $\mathrm{kPa}$ undergoing Bjerknes forces and an isolated bubble driven at $P_{a}=160 \mathrm{kPa}$, the maximum and the minimum radius correspond, thus providing consistency between the present work and Ref. [2].

The interaction with the other bubbles strongly influences the radical production even before the inception of the motion. In Fig. 8.12 we show the maximum number of $\mathrm{OH}$ radicals produced per cycle in one bubble of cluster 1 as function of the distance between the pits. When the distance between the pits decreases, so does the radical production, because the interaction with the neighboring bubbles becomes stronger, therefore damping the oscillations and decreasing the temperature at collapse. For the same reason, for a fixed number of bubbles, smaller clouds have lower radical production (Fig. 8.12), as the bubbles are closer. Similarly, for a fixed cluster 


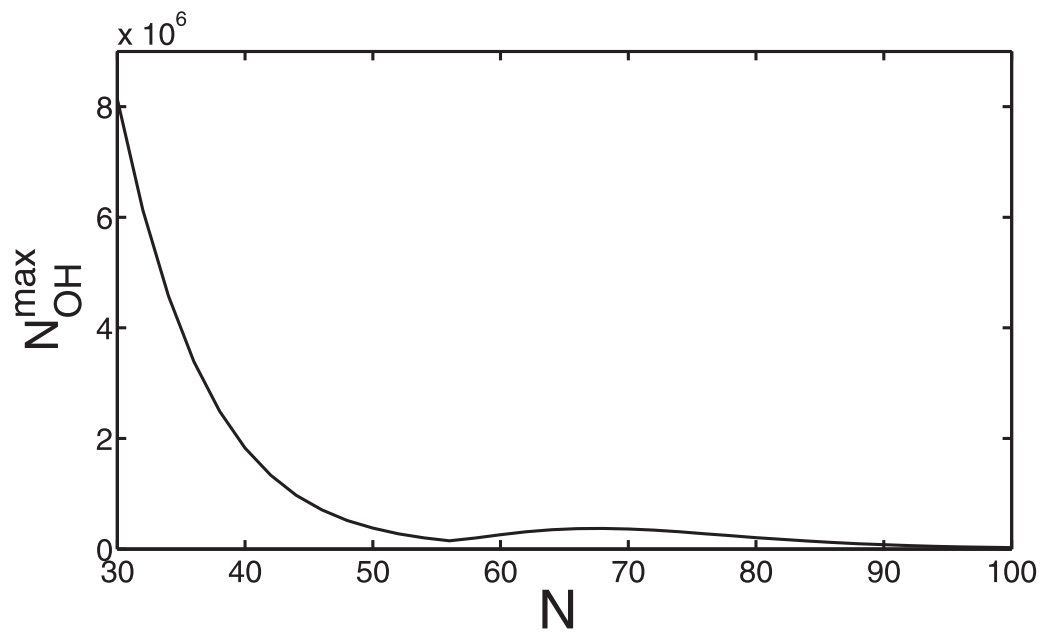

Figure 8.13: Maximum number of $\mathrm{OH}$ radicals in one acoustic cycle for a bubble of cluster 1 in the unperturbed position $(\delta=0 \mu \mathrm{m})$ as function of the number of bubbles. Parameters: $R_{c}=100 \mu \mathrm{m}, d=1000 \mu \mathrm{m}, R_{0}=3 \mu \mathrm{m}, f=200 \mathrm{kHz}, P_{a}=$ $170 \mathrm{kPa}$.

size, raising the number of bubbles reduces the chemical production (see Fig. 8.13). However, in the range between 50 and 100 bubbles, there is a local maximum at $N \sim 70$. This could provide an explanation to the experimental observation that, above a certain threshold, a further increase of the applied acoustic power does not enhance the radical production [2].

\subsection{Conclusions}

In the present work we theoretically studied the interactions between bubbles clusters generated from ultrasonically driven silicon etched micropits [2]. We addressed the transition between the three different behaviors observed in Ref. [2] at increasing acoustic power: clusters sitting upon their own pit, clusters pointing towards each other and clusters migrating towards the center point of the pits array. We considered each cluster as a point object and we examined the secondary Bjerknes forces acting upon it. These forces depend on the displacement of the cluster from the pit. While the cluster-cluster force is always attractive, in the considered parametric range, at high driving the pit-cluster force can also become repulsive at some points very close the pit, thus favoring the inception of the motion. Given the driving frequency and 
the size of the bubbles and the pits, this is in contrast with the predictions of the linear theory and it has to be ascribed to the nonlinearity of the phenomenon [9, 16, 21]. We found that it always exists a barrier, generally coinciding with the maximum attractive force of the pit, that needs to be overcome for the clusters merging to take place. This barrier is located at a distance of the order of the cluster radius, in agreement with experimental observations. The $P_{a}$ required for the cluster to escape the trapping force of the pit is consistent both with the measured ones and with the effective pressures that we extracted from the bubble dynamics in our previous work [2]. As the distance between the pits increases, the $P_{a}$ for merging also increases, up to a certain limiting distance, where the cluster cannot escape from the pits. For practical purposes, this could be regarded as an optimal distance between the pits for efficient sonochemical reactors design, where the number of the pits (i.e. of the bubbles) should be the maximum possible per unit area, but still avoiding the merging as it lowers the radical production [2] and enhances the erosion of the reactor walls [4].

We showed that the key to the transition to merging clusters relies on the influence of the nonlinear bubble oscillations and not on the increasing number of bubbles at increasing powers, as the $P_{a}$ for merging decreases when $N$ increases, but only until a certain threshold, after which it basically becomes constant. However, a non-direct influence of the number of bubbles can be present, because the $P_{a}$ for transition decreases with the size of the cluster. As strongly driven bubbles tend to form stable pairs rather than to coalesce [16], we can expect that increasing the number of generated bubbles will also increase the clusters radii. This dependence of the cluster dimension on the number of bubbles should be addressed in future works, for a more complete understanding of the phenomenon.

We also showed that the bubble size has an influence on the driving pressure required for merging, which presents a minimum for clusters with bubbles of $10 \mu \mathrm{m}$. We can therefore expect that the merging and the consequent generated flow are first initiated by the bubbles of this size, with some shift towards the smaller bubbles once that also inertia and drag come into play.

Finally, we examined the sonochemical production and we found that neglecting the Bjerknes forces will lead to a consistent overestimate of the number of radicals produced. This happens because the interaction with the neighbors dampens the oscillations of the bubble, reducing the temperature at collapse and also the resonance frequency. Since these interactions exhibit an inverse proportionality with the distance between the bubbles, smaller size of the clusters and shorter distances between the pits as well as higher number of bubbles strongly decrease the radical production, even before the merging takes place. This could be the key to explain the experimental observation that increasing the power after a certain threshold does not improve 
the sonochemical production. For practical purposes, the efficiency of a sonochemical reactor could benefit from a medium power operating condition instead of high power and a distance between the pits substantially larger, in order to prevent cluster merging, which reduces the chemical yield. 


\section{References}

[1] D. Fernández Rivas, A. Prosperetti, A. G. Zijlstra, D. Lohse, and H. J. G. E. Gardeniers, "Efficient sonochemistry through microbubbles generated with micromachined surfaces", Angew. Chem. Int. Ed. 49, 9699-9701 (2010).

[2] D. Fernández Rivas, L. Stricker, A. Zijlstra, H. Gardeniers, D. Lohse, and A. Prosperetti, "Ultrasound artificially nucleated bubbles and their sonochemical radical production", Ultrason. Sonochem. 20, 510-524 (2013).

[3] N. Bremond, M. Arora, C. D. Ohl, and D. Lohse, "Controlled multibubble surface cavitation", Phys. Rev. Lett. 96, 224501 (2006).

[4] D. Fernández Rivas, B. Verhaagen, J. R. T. Seddon, A. G. Zijlstra, L.-M. Jiang, L. W. M. van der Sluis, M. Versluis, D. Lohse, and H. J. G. E. Gardeniers, "Localized removal of layers of metal, polymer, or biomaterial by ultrasound cavitation bubbles", Biomicrofluidics 6, 034114 (2012).

[5] V. Bjerknes, Fields of forces (Columbia University Press, New York) (1906).

[6] T. G. Leighton, The acoustic bubble (Academic Press, London) (1994).

[7] F. G. Blake, "Onset of cavitation in liquids", Ph.D. thesis, Harvard University (1949).

[8] L. A. Crum, J. Acoust. Soc. Am. 57, 1363-1370 (1975).

[9] H. N. Og̃uz and A. Prosperetti, "A generalization of the impulse and virial theorems with an application to bubble oscillations", J. Fluid Mech. 218, 143-162 (1990).

[10] N. A. Pelekasis and J. A. Tsamopoulos, "Bjerknes forces between two bubbles. Part 1. Response to a step change in pressure", J. Fluid Mech. 254, 467-499 (1993).

[11] N. A. Pelekasis and J. A. Tsamopoulos, "Bjerknes forces between two bubbles. Part 2. Response to an oscillatory pressure field", J. Fluid Mech. 254, 501-527 (1993).

[12] A. A. Doinikov, "Bjerknes forces between two bubbles in a viscous fluid", J. Acoust. Soc. Am. 106, 3305-3312 (1999).

[13] A. A. Doinikov, "Mathematical model for collective bubble dynamics in strong ultrasound fields", J. Acoust. Soc. Am. 116, 821-827 (2004). 
[14] K. Yasui, Y. Iida, T. Tuziuti, T. Kozuka, and A. Towata, "Strongly interacting bubbles under an ultrasonic horn”, Phys. Rev. E 77, 016609-1 (2008).

[15] A. Prosperetti, "Bubble phenomena in sound fields: part two", Ultrasonics 22, 115-124 (1984).

[16] R. Mettin, I. Akhatov, U. Parlitz, C. D. Ohl, and W. Lauterborn, "Bjerknes forces between small cavitation bubbles in a strong acoustic field", Phys. Rev. E 56, 2924-2931 (1997).

[17] A. A. Doinikov, "Viscous effects on the interaction force between two small gas bubbles in a weak acoustic field", J. Acoust. Soc. Am. 111, 1602-1609 (2002).

[18] A. A. Doinikov, "Translational motion of two interacting bubbles in a strong acoustic field", Phys. Rev. E 64, 026301 (2001).

[19] A. Harkin, T. J. Kaper, and A. Nadim, "Coupled pulsation and translation of two gas bubbles in a liquid", J. Fluid Mech. 445, 377-411 (2001).

[20] N. A. Pelekasis, A. Gaki, A. Doinikov, and J. A. Tsamopoulos, "Secondary bjerknes forces between two bubbles and the phenomenon of acoustic streamers", J. Fluid Mech. 500, 313-347 (2004).

[21] K. Yoshida, T. Fujikawa, and Y. Watanabe, "Experimental investigation on reversal of secondary bjerknes force between two bubbles in ultrasonic standing wave", J. Acoust. Soc. Am. 130 135-144, 727-734 (2011).

[22] R. Toegel, S. Hilgenfeldt, and D. Lohse, "The effect of surfactants on single bubble sonoluminescence”, Phys. Rev. Lett. 84, 2509-2512 (2000).

[23] R. Toegel, S. Hilgenfeldt, and D. Lohse, "Suppressing dissociation in sonoluminescing bubbles: The effect of excluded volume", Phys. Rev. Lett. 88, 034301 (2002).

[24] R. Toegel and D. Lohse, "Phase diagrams for sonoluminescing bubbles: A comparison between experiment and theory", J. Chem. Phys. 118, 1863 (2003).

[25] L. Stricker, A. Prosperetti, and D. Lohse, "Validation of an approximate model for the thermal behavior in acoustically driven bubbles", J. Acoust. Soc. Am. 130, 3243-3251 (2011).

[26] Z. Zeravcic, D. Lohse, and W. van Saarloos, "Collective oscillations in bubble clouds”, J. Fluid Mech. 680, 114-149 (2011). 
[27] A. Prosperetti, "Bubble phenomena in sound fields: part one", Ultrasonics 22, 69-77 (1984).

[28] H. Gelderblom, A. G. Zijlstra, L. van Wijngaarden, and A. Prosperetti, "Oscillations of a gas pocket on a liquid-covered solid surface", Phys. Fluids 24, 122101 (2012).

[29] A. Zijlstra, "Acoustic surface cavitation", Ph.D. thesis, University of Twente (2011).

[30] M. S. Plesset, "Comment on 'Sonoluminescence from water containing dissolved gases' (J. Acoust. Soc. Am. 60, 100-103 (1976))”, J. Acoust. Soc. Am. 62, 470 (1977).

[31] M. S. Plesset and A. Prosperetti, "Bubble dynamics and cavitation", Annu. Rev. Fluid Mech. 9, 145-185 (1977). 


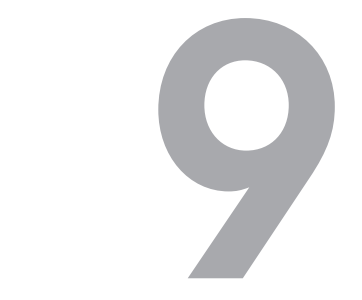

Conclusions

\subsection{Conclusions and outlook}

Though sonochemistry is a promising field for industrial application (eg. chemical synthesis, textile processing, water cleaning), its practical use has always suffered from low efficiency and difficult controllability. The ultimate task of our project was to address this two issues. The present work was intended as a theoretical study of a new generator multibubble sonochemical reactors recently developed by FernandezRivas et al. [1]. Such a device is based on the principle that cavitation can be enhanced by the presence of artificial crevices micromachined over a silicon substrate. When the substrate is introduced inside the liquid cuvette of a standing-wave sonochemical reactor, gas pockets are trapped inside the pits. Through acoustic excitation, the pits can eject a manifold of bubbles which start to interact with each other, forming clusters and complicate patterns as the power increases. Though they do not survive more than few acoustic cycles, due to recombination and splitting phenomena, still they have been proved to be sonochemically active [1]. The chemical yield of this kind of reactors is 10 times higher than the equivalent preexisting reactors thus representing the state of the art $[2,3]$.

Even disregarding the interactions between the bubbles, i.e. considering each bubble as an isolated reactor, the whole process is still very complex, from the modelling point of view. For practical purposes, such as the estimate of the chemical 
production of the bubble population or a parametric study of the optimal operating conditions, it was necessary to develop a simplified approach. Given the strict interdependence of the temperature and the chemical output, through Arrhenius law, the main concern was the thermal evolution of the bubble. In Chap. 2 we have validated a simplified ODE model based on the boundary layer approximation of Ref. [4], by comparing it with a full PDE model providing the solution to the heat advectiondiffusion equation following the approach of [5]. Though the latter accounts for the whole temperature distribution both in the bubble and in the liquid, while the other treats the bubble as a spatially homogeneous system, we have found that, in the parametric range where shape stability holds, the two models were in very good agreement.

In Chap. 3 we have characterized a sonochemical reactor of the kind of Ref. [1], providing the experimental bubble size distributions at different power settings and number of pits, and their temporal evolution. We used the simplified model validated in Chap. 2 to give an estimate of the radical production. A major difficulty derived from the fact that the presence of the bubbles altered the pressure field in a way which was not measurable with the standard experimental equipment. We overcame this problem, by fitting the experimental and the calculated PDF of the radii for a range of pressure amplitudes and minimizing the cumulative squared error between the two. We used therefore the bubbles as a pressure sensor themselves and we provided an estimate of the effective forcing pressure that they were feeling. We found that, in the two- and three-pit cases the effective pressure dropped when bubbles started to migrate towards the center point of the pit array. Through simulations, we could also overcome another limitation of the experimental apparatus, giving a description of the temporal evolution of the smaller bubbles (below observability), compatible with the experimental PDF. This result was particularly relevant, as calculations showed that the major part of $\mathrm{OH}$. radical production came from the smaller bubbles (around 5 $\mu \mathrm{m}$ radius). As a general trend, the calculated chemical rate increased monotonically with the effective pressure.

In the attempt to give practical indications for the optimal working conditions of the sonochemical reactor, in Chap. 4 we developed an extensive parametric study. We considered each bubble as an isolated reactor and we examined the radical production during the early transient, using the model validated in Chap. 2. We chose this time frame in order to match the operating conditions of the reactor, where the bubble life was of few acoustic cycles. We found that the chemical production was strongly affected by two factors: the gas temperature and the composition of the bubble at collapse. We underlined the importance of water vapor, $\mathrm{H}_{2}$ and $\mathrm{O}_{2}$ dissociating reactions and their feedback on the temperature. The dissociation of water is strongly 
endothermal and reduces the temperature of the bubble, while the dissociation of $\mathrm{H}_{2}$ and $\mathrm{O}_{2}$ are initil steps of globally strongly exothermal chain reactions which abruptly increase the temperature, enhancing, in return, other chemical reactions. We found that, mixtures of $\mathrm{H}_{2}$ and $\mathrm{O}_{2}$ with the stoichiometric composition of water give origin to a peak in temperature, whenever the activation energy for $\mathrm{H}_{2}$ and $\mathrm{O}_{2}$ dissociation is fulfilled. This reproduces the well known chemical explosive behavior of such mixtures and provides a strong evidence of the reliability of the adopted model. For practical purposes, we found that, at low frequency (below $20 \mathrm{kHz}$ ), a good strategy to enhance the radical production is to use stoichiometric mixtures of $\mathrm{H}_{2}$ and $\mathrm{O}_{2}$, while, at high frequency (around $200 \mathrm{kHz}$ ), it is preferable to add a large amount of argon $(\sim 90 \%)$, to increase the adiabatic index of the mixture and therefore the peak temperature. From the study of the size of the most active bubbles, we discovered that another strategy to scale up sonochemistry might be to promote the nucleation of big bubbles in low frequency reactors and small bubbles in high frequency reactors, for example by changing the size of the pits. We found that the optimal operating range for frequencies is between $20 \mathrm{kHz}$ and $40 \mathrm{kHz}$, while for the liquid temperature heating to $60^{\circ} \mathrm{C}$ could be beneficial.

In view of a future development of a complete PDE model for a single cavitation bubble, including temperature and mass distribution as well as evaporation/condensation and chemical reactions, in Chap. 5 and Chap. 6 we have outlined a preliminary study, addressing mass diffusion and evaporation. In Chap. 5, we developed a PDE model for a spherical cavitation bubble including the advection-diffusion equations both for the heat inside the bubble and for the mass of gas inside the liquid. After validation by comparison with the analytical solution in a test case, we also compared the numerical results with the experimental ones for the evolution of a bubble growing purely by mass diffusion attached to a pit. As our model relies on the assumption of spherical symmetry, in the very first phases of the nucleation, a direct comparison was not possible, due to the strong asymmetry of the process. However, after sphericity was regained, a very good agreement was found, upon introduction of a geometric correction inside the model, to account for the disruption of the boundary layer next to the wall. With this correction, the simulations were able to reproduce the non-square-root like evolution of the bubble found in the experiments, particularly interesting because it differs from the classical square-root behavior of a bubble growing purely by mass diffusion in a infinite medium [6].

In Chap. 6 we developed a PDE code including both heat and mass diffusion inside the liquid and evaporation/condensation at the bubble wall. We verified that, in the limiting case of pure gas diffusion and pure vapor bubble, it gave the same results of Chap. 4 and Ref. [7], respectively. As a further validation, we used such code 
to track the growth of a perfluoropentane (PFP) vapor bubble nucleated and driven with ultrasound, inside a PFP droplet, contained in a water medium superheated with respect to the PFP boiling point. We found an agreement within a $10 \%$ error between numerics and experiments. Simulations underlined the fundamental role of gas diffusion during acoustic forcing. Though the bubble dynamics was entirely governed by evaporation and condensation processes, as the rate between gas and vapor mass was around $10^{-2}$, a minimum amount of gas ( $\sim 10 \%$ of the air solubility in PFP) was necessary in order to allow the bubble to survive the first collapse.

After having addressed the behavior of a single bubble, we turned to the formation mechanism of the bubbles from the pits. In Chap. 7 we developed a code to track the dynamics of an air pocket trapped inside a pit, namely the oscillating meniscus from which the bubbles originate. This code was based on a level set method coupled to a projection method in order to solve the Navier-Stokes equations in the liquid and to track the evolution of the velocity and the pressure field around the pit and the related interface displacement. We validated it by comparison with the analytic solution both from the mixed boundary problem of the flat meniscus used as test case and the results of Ref. [8] for the limiting cases of Stokes and potential flow. We found an agreement within the $5 \%$ of error. In the case of free oscillations with the inclusion of both inertial and viscous effects, the behavior of the pit approached the Stokes flow behavior, as the hydrostatic pressure increased. Moreover, in the regime of large amplitude oscillations, the damping was slightly lower respect to the case of small amplitude oscillations, but the eigenfrequency was the same. In the case of the forced oscillations with a flat equilibrium condition of the interface, regardless the initial perturbation, the first mode of oscillation was always selected after very few cycles and no bubble formation appeared, when the system was driven at the main resonance. Therefore we concluded that the mechanism of formation of the bubbles is related to something else than the main resonance and the connected abrupt response of the system. Possible candidates are the excitation of higher modes and a mass rectified diffusion-like mechanism [9], through the change of the equilibrium configuration of the interface that it induces. This aspects will have to be addressed in future developments.

As a conclusion of the work, in Chap. 8, we studied the collective behavior of the bubbles generated in the sonochemical reactor, in the two-pit configuration. For such purpose, we used an extended version of the simplified model validated in Chap. 2, including the secondary Bjerknes forces acting upon a bubble from the bubbles of both the same and the opposite cluster, from the pits and from the mirror clouds, given the presence of the silicon substrate. In particular, we investigated the different behaviors observed in the experiments at increasing acoustic power [3]: clusters 
sitting upon their own pits, clusters pointing towards each other and clusters migrating towards the center of the pit array. As cluster merging is associated to a reduced sonochemical yield [1], an optimized design of a sonochemical reactor is expected to avoid this situation, still providing a high number of pits (i.e. of bubbles). We calculated the maximum displacement of the cluster before transition and the required driving amplitude, verifying that they were in the same range of the experimental ones and consistent with the pressure values estimated in Chap. 3. As the distance between the pits increased, the $P_{a}$ for merging also increased, up to a certain limiting distance, where the cluster could not escape the pits. For practical purposes, this could be regarded as an optimal distance between the pits for efficient sonochemical reactors design. We showed that the key to the transition relies on the influence of the nonlinear bubble oscillations while it is almost independent from the number of bubbles. However, the number of bubbles can have an indirect influence, as the $P_{a}$ for transition decreases with the size of the cluster and a higher number of bubbles can easily turn into a larger bubble cluster. This dependence of the cluster dimension on the number of bubbles should be addressed in future works. We saw that the Bjerknes forces dramatically decreased the radical production, as they dampened the bubble oscillations, reducing the temperature at collapse and the resonance frequency. In particular, reduced size of the clusters and shorter distances between the pits as well as higher number of bubbles turned into a lower radical production, even before the merging took place. This could be the key to explain the experimental observation that increasing the power after a certain threshold does not improve the sonochemical production but can even reduce it [1]. For practical purposes, the efficiency of a sonochemical reactor could benefit from a medium power operating condition and a distance between the pits substantially larger respect to the one required for clusters merging.

Though the present Thesis has investigated many theoretical aspects of the sonochemical reactors presented in Refs. [1-3], still many things remain unclear, such as the precise mechanism of formation of the bubbles and the complicate interaction between the clusters in the case with more than two pits. Also in the case of two pits a high level of simplification has been adopted assuming monodispersity of the clusters, equal size of the bubbles, constant-time cluster size and constant number of bubbles. A more complete future development should eliminate these hypothesis. We have confidence that, through a deeper understanding of the collective bubble dynamics, a further significant increase in the sonochemical yield could be achieved. Therefore, before the device can be used in large-scale industrial processes, these aspects should be clarified. 


\section{References}

[1] D. Fernández Rivas, A. Prosperetti, A. G. Zijlstra, D. Lohse, and H. J. G. E. Gardeniers, "Efficient sonochemistry through microbubbles generated with micromachined surfaces", Angew. Chem. Int. Ed. 49, 9699-9701 (2010).

[2] D. Fernández Rivas, “Taming acoustic cavitation", Ph.D. thesis, University of Twente (2012).

[3] D. Fernández Rivas, L. Stricker, A. Zijlstra, H. Gardeniers, D. Lohse, and A. Prosperetti, "Ultrasound artificially nucleated bubbles and their sonochemical radical production”, Ultrason. Sonochem. 20, 510-524 (2013).

[4] R. Toegel and D. Lohse, "Phase diagrams for sonoluminescing bubbles: A comparison between experiment and theory”, J. Chem. Phys. 118, 1863 (2003).

[5] V. Kamath, A. Prosperetti, and F. N. Egolfopoulos, "A theoretical study of sonoluminescence”, J. Acoust. Soc. Am. 94, 248-260 (1993).

[6] M. S. Plesset and S. A. Zwick, "The growth of vapor bubbles in superheated liquids”, J. Appl. Phys. 25, 493-499 (1954).

[7] Y. Hao and A. Prosperetti, "The dynamics of vapor bubbles in acoustic pressure fields”, Phys. Fluids 11, 2008-2019 (1999).

[8] H. Gelderblom, A. G. Zijlstra, L. van Wijngaarden, and A. Prosperetti, "Oscillations of a gas pocket on a liquid-covered solid surface”, Phys. Fluids 24, 122101 (2012).

[9] D. Y. Hsieh and M. S. Plesset, "Theory of rectified diffusion of mass into gas bubbles", J. Acoust. Soc. Am. 33, 206-215 (1961). 


\section{Summary}

Sonochemistry is the use of cavitation for achieving a chemical conversion. When microbubbles are driven in the nonlinear regime, localized extreme temperatures (up to $10000 \mathrm{~K}$ ) and pressures (up to 1000 bar) can be reached upon collapse, the surrounding liquid remaining ambient, thus giving origin to intriguing phenomena, such as light emission (sonoluminescence) and high temperature chemical reactions (sonochemistry). These reaction products then diffuse outside the bubble and dissolve inside the surrounding liquid. Due to their unstable nature, they are highly reactive and have therefore a vast potential for technological applications, eg. chemical synthesis, water cleaning, cells disruption and textile processing. However, sonochemical reactors are known to suffer from a lack of efficiency and controllability, which has until now prevented the large-scale employment of this technology. When we started the present study the challenge was to improve the efficiency of a sonochemical reactor, by reducing its dimensions to a micrometric scale, still retaining the possibility to control the precess. In this frame David Fernández Rivas (Ph.D. thesis 2012) developed a new type of sonochemical microreactor, based on the principle that artificial micromachined crevices, immersed inside a liquid bath and ultrasonically driven, can produce a manifold of bubbles. This device turned out to have a chemical yield 10 times higher than the equivalent existing immersed-bath reactors, thus representing the state of the art. The present Thesis was intended as a complementary theoretical study to the experimental work developed by Fernández Rivas (Ph.D. thesis 2012). Here, we have addressed the main aspects of the observed process, both from the technological and the fundamental point of view, namely the radical production, the bubble formation mechanism and the bubble-bubble interaction. The first problem that we encountered was the high complexity of the phenomenon. Therefore a consistent part of the modelling effort was devoted to the development and the validation of a simplified model for a single cavitation bubble. This instrument allowed us both to make predictions for the optimal working ranges of the sonochemical reactor and to overcome some of the limitations of the experimental apparatus. For example, using the bubbles themselves as pressure sensors, we were able to estimate the local pressure that they were experiencing inside the cloud. This simplified approach 
also allowed us to gain some knowledge on the fundamental mechanisms governing the sonochemical conversions, such as the endothermal water vapor dissociating reactions and the highly exothermal chain reactions triggered by the $\mathrm{H}_{2}$ and $\mathrm{O}_{2}$ dissociation. An interesting result was that small bubbles made of a stoichiometric $\mathrm{H}_{2}-\mathrm{O}_{2}$ mixture were found to reproduce the well known macroscopic explosive behavior of the stoichiometric mixtures. This theoretical prediction will need experimental verification, in the upcoming future, but, proved true, it would asses a nice link between the macroscopic and the microscopic phenomena.

Another part of our work was devoted to understand the bubble formation mechanism, through the study of the oscillations of a gas pocket trapped inside a micropit. We saw that, by considering only the dynamics, in the parametric range that we are concerned with, even in the large amplitude oscillations regime, its behavior remains close to the small linear oscillations regime, as about the eigenfrequency and the damping coefficient. For e.g., in the case of large amplitude free oscillations, the deformation of the interface does not turn into surface instabilities that could eventually eject bubbles, but tends towards the first oscillating mode after very few cycles. The same applies in the case of forced oscillations when the meniscus has a flat equilibrium condition and it is driven at the main resonance frequency. Therefore we concluded that the bubble formation mechanism may be connected to other phenomena than merely the excitation of the main resonance (e.g. a rectified diffusion-like mechanism, through the change of the equilibrium condition, or the excitation of higher modes). This fundamental aspect still needs to be investigated in future studying.

In conclusion, we addressed the bubble dynamics, investigating the conditions for which the bubble clusters produced from two adjacent pits tend to migrate towards each other and eventually to merge, as an effect of the secondary Bjerknes force. This condition has been proved to be associated with a drop of the sonochemical yield and therefore, in efficient sonochemical reactors, the pits should be enough far to prevent it. We found that high numbers of bubbles in the clusters should be avoided as well: though they do not influence the transition to merging clusters, they still provide a dramatic decrease of the radical production. Therefore medium operating powers should be preferred, instead of high powers.

We have confidence that the knowledge that we gained with the present work could help to improve the efficiency of this kind of sonochemical microreactors. However, a deeper studying is still required to address the fundamentals aspects of the bubble formation, the flow generated by the bubbles motion as well as their complex interactions. 


\section{Samenvatting}

In de sonochemie wordt gebruik gemaakt van cavitatie om chemische reacties te bewerkstelligen. Wanneer microbellen die worden aangedreven in het niet-lineaire regime ineenklappen ontstaan locaal zeer hoge temperaturen (tot $10.000 \mathrm{~K}$ ) en drukken (tot 1000 bar). Deze extreme condities leiden tot bijzondere fenomenen, zoals de uitzending van licht (sonoluminescentie) en het optreden van chemische reacties (sonochemie). De reactieproducten diffunderen vervolgens de bel uit en lossen op in de omringende vloeistof. Doordat deze reactieproducten instabiel zijn, zijn ze zeer reactief en kunnen ze gebruikt worden voor vele toepassingen zoals chemische synthese, waterzuivering, het perforeren van celwanden en textielverwerking. Sonochemische reactoren kunnen echter nog niet op grote schaal gebruikt worden, omdat hun efficiëntie en controleerbaarheid te wensen overlaat.

De uitdaging van de huidige studie lag in het vergroten van de efficiëntie van de sonochemische reactor door zijn afmetingen terug te brengen tot de micrometerschaal en tegelijkertijd controle over het proces te houden. Hiertoe is een nieuw type sonochemische reactor ontwikkeld door David Fernández Rivas (Ph.D. thesis 2012). De werking van deze reactor is gebaseerd op kunstmatig aangebrachte holtes op substraten die in water worden ondergedompeld. In deze holtes vormen zich bellen, die kunnen worden aangedreven met ultrageluid. Deze reactor bleek een chemische opbrengst te hebben die tien keer zo groot was als die van de bestaande reactors.

Deze thesis is bedoeld als aanvullende theoretische studie om het experimentele werk van Fernández Rivas te complementeren. We hebben aandacht besteed aan de meest belangrijke processen in de reactor, te weten de productie van radicalen, de belvorming en de interactie tussen bellen. Hierbij hebben we zowel de toegepaste als de fundamentele aspecten belicht. Het eerste probleem waar we mee te maken kregen was de enorme complexiteit van het fenomeen. Om het probleem goed te kunnen bestuderen hebben we daarom eerst een versimpelde situatie van een enkele cavitatiebel bekeken. Een groot deel van het werk besloeg het modeleren van deze enkele bel en het valideren van dit model. Met behulp van dit simpele model konden we de optimale condities bepalen voor de werking van de sonochemische reactor. Door bijvoorbeeld de bellen zelf als druksensoren te gebruiken waren we in staat 
om de locale druk te schatten waaraan zij blootstonden. Onze aanpak verschafte ook meer inzicht in de fundamentele processen achter de chemische reacties, zoals bijvoorbeeld de endotherme dissociatie van waterdamp en de sterk exotherme kettingreacties volgend op de dissociatie van $\mathrm{H}_{2}$ en $\mathrm{O}_{2}$. Een interessant resultaat was dat kleine bellen bestaande uit het stoichiometrische mengsel $\mathrm{H}_{2}-\mathrm{O}_{2}$ het bekende macroscopische explosieve gedrag van stoichiometriche mengsels konden reproduceren. Deze theoretische voorspelling moet nog experimenteel gevalideerd worden, maar, als het klopt zou het een mooie link tussen macroscopische en microscopische fenomenen vormen.

Een ander deel van het werk ging over belvormingmechanismen. Hiertoe bestuurden we de trillingen van een gasbel die is ingevangen in een microscopische holte. We zagen dat het dynamische gedrag van de bel, in termen van haar eigenfrequentie en dempingcoëfficiënt, dicht in de buurt van het lineaire trillingsregime blijft, zelfs als de amplitude van de trillingen toeneemt. Voor vrije trillingen met grote amplitude blijft het grensvlak van de bel stabiel waardoor de uitstoot van bellen niet plaatsvindt. Hetzelfde geldt in het geval van gedwongen trillingen wanneer de meniscus een vlakke evenwichtstoestand heeft en wordt aangedreven met de eerste resonantiefrequentie. We zagen dat de trillingen van het grensvlak na een paar cycli kunnen worden beschreven in de eerste oscillatiemodus. Om deze reden concludeerden we dat er andere fenomenen dan respons op de eerste resonantiefrequentie ten grondslag liggen aan belvorming (zoals bijvoorbeeld gerectificeerde diffusie of de excitatie van hogere modi). Dit fundamentele effect dient nog verder te worden onderzocht.

In dit onderzoek hebben we gekeken naar beldynamica en de condities waaronder migratie en samensmelting van belclusters uit naburige holtes optreedt ten gevolge van de secundaire Bjerkneskracht. Deze condities bleken sterk samen te hangen met een afname van de sonochemische opbrengst. Om deze migratie en samensmelting te voorkomen en de efficiëntie van de sonochemische reactor te verhogen dienen de holtes ver genoeg uit elkaar geplaatst te worden. We hebben ontdekt dat het ook belangrijk is om grote aantallen bellen in de clusters te vermijden: hoewel het geen invloed heeft op het samensmelten, leidt het wel tot een enorme afname in de productie van radicalen. Om deze reden is het beter om de reactors bij gematigd vermogen te laten werken, in plaats van bij hoog vermogen.

De kennis die verkregen is met deze studie kan gebruikt worden om de efficiëntie van sonochemische reactoren te verhogen. Wel is er vervolgonderzoek nodig om de fundamentele aspecten van belvorming, de stroming die gegenereerd wordt door beweging van bellen en de complexe interacties tussen bellen beter te begrijpen. 


\section{Acknowledgements}

This work is part of the research program of the Technology Foundation STW, which is financially supported by the Nederlandse Organisatie voor Wetenschappelijk Onderzoek (NWO) and the Technology Programme of the Ministry of Economic Affairs, The Netherlands, to which I am thankful.

I am grateful to Professor Detlef Lohse, who gave me the great opportunity to develop this work and taught me what it means to work in a scientific team, for his constant support and advice, especially during the last months, when they were more necessary.

I thank Professor Andrea Prosperetti, for his precious contribution and constant help throughout this work and for giving me the chance to visit him in Baltimore and to study in Johns Hopkins University.

I would also like to thank Professor Leen van Wijngaarden, for insightful scientific discussions, which were always a source of great pleasure and inspiration.

Many thanks to David Fernández Rivas, with whom we developed a great part of the present work, for his energetic contribution and help. Many thanks also to Professor Han Gardeniers for the fruitful collaboration and the scientific contribution during these four years.

For the work of Chap. 3, I would like to acknowledge S. Schlautmann (microfabrication) and J. Martinez (image processing) for their technical support, Prof. F. Grieser and Prof. M. Ashokkumar for fruitful discussions. The advice from Prof. C.-D. Ohl and Dr. Tandiono on the experimental pressure measurements was highly appreciated.

For the work of Chap. 4, I would like to acknowledge Dr. Vitaly Svetovoy for fruitful discussions.

For the work of Chap. 6, I would like to acknowledge Oleksandr Shpak and Dr. Michel Verslius, for their scientific contribution, Mario Fabiilli for preparing PFP droplet emulsions and Tom Kokhis, Ying Luan, Brian Fowlkes and Nico de Jong for their help during the experimental measurements.

For the work of Chap. 7, I would like to thank Dr. Edip Can, for his patient guidance and Dr. Paolo Oresta for crucial scientific discussions, without which some 
problems would be still unsolved.

For the work of Chap. 8 I would like to acknowledge Dr. Benjamin Dollet for his scientific contribution.

A special thanks goes to my office mates, present and former, Álvaro Gómez Marin, Koen Winkels, Henry Lhuissier and Claas Willem Visser for nice and fruitful discussions, both scientific and personal. It was a real pleasure to share these years with you. Many thanks also to Hanneke Gelderblom for her scientific and personal support.

Many thanks to the technical staff, Bas Banschop, Martin Bos and Gert-Wim Bruggert and to Joanita Leferink in particular, who always helped me with practical and bureaucratic issues with great kindness and efficiency.

I thank my students of the course of Heat and Mass Transfer, because I learned a lot through their questions.

Many thanks to Marco and to my friends for their constant presence and support. Last but not least, a great thanks to my parents, in whom I found invaluable support, whenever I needed it, despite the distance. 


\section{About the author}

Laura Stricker was born the $16^{\text {th }}$ of June 1983 in Turin, Italy. She studied Mechanical Engineering at the 'Politecnico di Torino', where she got her batchelor degree in 2005 with a thesis from the title "Dynamical evolution of close parametrical curves", under the supervision of Prof. Luca Ridolfi, Prof. Luigi Butera and Dr. Paolo Perona. She got her master degree in 2008, under the supervision of Prof. Lamberto Rondoni, with a thesis from the title "Vibrations in mechanical systems in conditions of equilibrium and non-equilibrium, with applications to gravitational waves detectors", inside the context of the European project RareNoise for the detection of gravitational waves. In 2009 she took her state examination to qualify for the profession of Engineer, according to the Italian law. In 2009 she joined the Physics of Fluid group at the University of Twente, as a Ph.D. student, under the supervision of Prof. Detlef Lohse and Prof. Andrea Prosperetti. 
